\title{
Spectroscopy of Binaries in Globular Clusters
}

\author{
Dissertation \\ for the award of the degree \\ "Doctor rerum naturalium" (Dr.rer.nat.) \\ of the Georg-August-Universität Göttingen \\ within the doctoral program PROPHYS \\ of the Georg-August University School of Science (GAUSS)
}

submitted by

Benjamin David Giesers

from Oldenburg

Göttingen, 2019 


\section{Thesis Commitee}

Prof. Dr. Stefan Dreizler

Sonnenphysik und Stellare Astrophysik, Institut für Astrophysik, Georg-August-Universität Göttingen, Germany

Dr. Sebastian Kamann

Star formation and stellar populations, Astrophysics Research Institute, Liverpool John Moores University, United Kingdom

Dr. Tim-Oliver Husser

Stellare Astrophysik, Institut für Astrophysik, Georg-August-Universität Göttingen, Germany

\section{Members of the Examination Board}

Reviewer: Prof. Dr. Stefan Dreizler

Sonnenphysik und Stellare Astrophysik, Institut für Astrophysik, Georg-August-Universität Göttingen, Germany

Second Reviewer: Prof. Dr. Ulrich Heber

Stellare Astrophysik, Dr. Karl Remeis Sternwarte, Friedrich-Alexander-Universität ErlangenNürnberg, Germany

Further members of the Examination Board:

Dr. Saskia Hekker

Stellar Ages and Galactic Evolution, Max-Planck-Institut für Sonnensystemforschung, Göttingen, Germany

Prof. Dr. Wolfram Kollatschny

Extragalaktische Astrophysik, Institut für Astrophysik, Georg-August-Universität Göttingen, Germany

Prof. Dr. Wolfgang Glatzel

Sonnenphysik und Stellare Astrophysik, Institut für Astrophysik, Georg-August-Universität Göttingen, Germany

PD Dr. Jörn Große-Knetter

Kern- und Atomphysik, II. Physikalisches Institut, Georg-August-Universität Göttingen, Germany

Date of the oral examination: 13 December 2019 


\section{Abstract}

The natural constants in the universe are adjusted so that dense and massive star forming regions can end up in gravitational bound star clusters, called globular clusters. These objects are found in all galaxies and are building blocks of galaxies and the universe. The kinematic properties of globular clusters cannot be explained by that of a population consisting only of single stars. In fact, an important aspect of these clusters are binary star systems, as they alter the dynamical evolution. To date, there are some estimates of the binary fraction of Galactic globular clusters, but very little is known about the properties of binary systems themselves. With their high mass, black holes can also have an important impact on the cluster evolution. However, black holes are extremely difficult to detect unless they are actively accreting.

In order to gain insights into the binary properties, such as orbital period, multi-epoch spectroscopic observations of individual stars in a given cluster are generally needed. However, classical spectrographs only offer limited capabilities when it comes to probing the crowded central regions of globular clusters where most of the binary systems are expected to be found. This is where the Multi Unit Spectroscopic Explorer (MUSE), a second generation instrument mounted on one of the Very Large Telescopes (VLTs), comes into play. With MUSE, the spectroscopy of individual stars in cluster cores became not only feasible, but also observationally efficient. This work makes use of multi-epoch MUSE observations of 27 Galactic globular clusters, including more than 380000 stars and 1400000 individual spectra, to derive radial velocities that allow the identification and characterisation of binary systems.

For the first time in astronomy, three binary systems, each composed of a black hole and a visible stellar companion, were discovered by this blind spectroscopic survey. A fit of a Keplerian orbit to these systems allowed us to infer the properties which are accessible by the radial velocity method. These findings helped to understand the retention fraction of black holes and how they affect the dynamical properties of globular clusters. The identification of these three black holes in NGC 3201 provided evidence that the cluster hosts an extensive subsystem of black holes including at least 40 objects. Black holes, as well as the binary systems, provide the cluster with an extra source of energy that explains why the core of NGC 3201 is not as dense as expected for a 12 Gyr old simple stellar population.

The pilot study on the globular cluster NGC 3201 shows what can be learned about the binary content of a globular cluster when using state-of-the-art observations and simulations. A new statistical method was developed to infer the binary probability of individual stars based on noisy and sparse radial velocities taking data from all stars of a given cluster into account. With the help of a sophisticated MOCCA globular cluster model of NGC 3201, the total binary fraction of $6.8 \%$, freed from many observational biases, was derived for this cluster. This is the most accurate estimate obtained to date. The best fitting MOCCA model also suggests that NGC 3201 was born with a large binary fraction $(\geq 50 \%)$ and that the present day binary population consists mainly of primordial binaries. For the first time in a study of globular clusters, Keplerian orbits to a significant sample of 95 binaries were obtained. The periods, eccentricities, and derived minimum companion masses of these systems give insights into the binary population of NGC 3201. The combination of Hubble Space Telescope (HST) photometry and literature data with the MUSE star sample of NGC 3201 revealed the binary nature and spectral properties of peculiar objects such as blue straggler stars, sub-subgiants, and eclipsing binaries. This showed a high blue straggler binary fraction of at least $58 \%$ and evidence for two 
blue straggler formation scenarios in NGC 3201. On the one hand, blue stragglers are formed involving mass transfer in a binary or triple star system. On the other hand, blue stragglers are formed by the coalescence of two stars following the encounter of two binary systems.

It is now known that most Galactic globular clusters do not consist of only one primordial stellar population, but have instead multiple populations that differ, for example, in elemental abundances. NGC 3201 shows two distinct populations and it was a logical step to compare the binary contents of these populations with each other. We found, that the binary fraction of the first population is significantly higher than the fraction of the second population. Previous studies have shown similar results for the outer regions of other clusters, but this work was the first to find such results in a cluster centre. This challenges some theories that expect the binary fractions of different populations in cluster centres to be the same. In the core of NGC 3201 the most plausible explanation is that the populations were formed with different primordial binary fractions, which is conceivable if the second population has been formed within and after the already formed first population. 


\section{Acknowledgments}

I would like to thank Stefan Dreizler very much for enabling me to explore the different aspects of astrophysics. He is responsible for the multitude of opportunities I had during my Bachelor, Master, and PhD projects. I also give special thanks to my additional supervisors Sebastian Kamann and Tim-Oliver Husser, not only for their helpful and patient support, but for all the work done beforehand, without which this work would not have been possible.

The opportunities allowed me to collaborate internationally with Guillem Anglada Escudé and Abbas Askar. Both have greatly contributed to the significance of my scientific work.

I would like to thank the MUSE consortium for building such a wonderful instrument and organising several inspiring meetings all over Europe with many helpful scientific discussions. I also like to thank ESO for the preparation of the press release (especially Richard Hook) and the unique experience at the Paranal Observatory, which I will never forget.

A special thanks goes to the co-authors of my papers, Abbas Askar, Guillem Anglada Escudé, Jarle Brinchmann, Peter Weilbacher, Marcella Carollo, Martin Roth, Martin Wendt, Lutz Wisotzki, Nate Bastian, Fabian Göttgens, Marilyn Latour, Tim-Oliver Husser, Sebastian Kamann, and Stefan Dreizler.

Finally, I would like to thank a selection of people who have accompanied me on my scientific journey: Jantje Freudenthal, Marvin Böhm, Wolfram Kollatschny, Roland Bacon, Rick Hessman, Arash Bahramian, Robert Mathieu, and Stan Lai.

Göttingen, October 2019

Benjamin Giesers 



\section{Contents}

1. Introduction 1

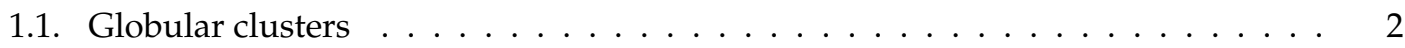

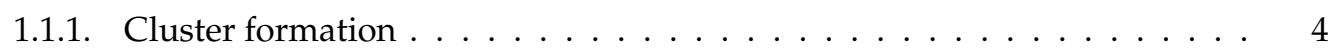

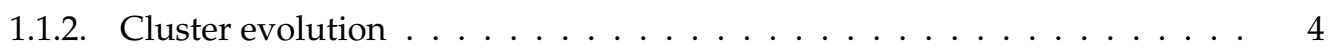

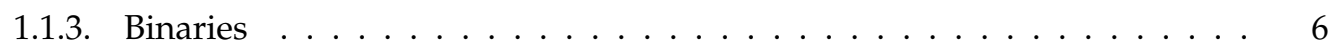

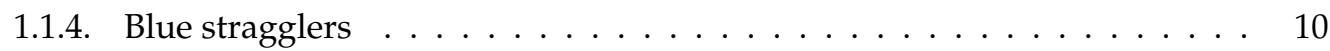

1.1.5. Black holes . . . . . . . . . . . . . . . . . . . . . . . 12

1.1.6. Multiple populations . . . . . . . . . . . . . . . . . . . . 14

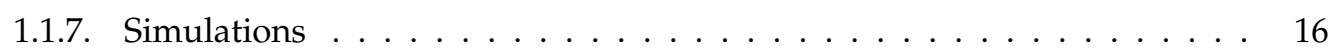

1.2. Multiple star systems and binaries . . . . . . . . . . . . . . . . . . . 17

1.2.1. Observational techniques . . . . . . . . . . . . . . . 18

1.2.2. The radial velocity technique of binary systems . . . . . . . . . . . . 19

1.3. Integral-field spectroscopy and MUSE . . . . . . . . . . . . . . . . . . 21

1.4. MUSE survey of globular clusters . . . . . . . . . . . . . . . . . 22

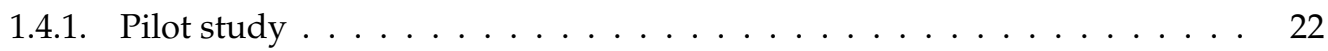

1.4.2. A stellar census of globular clusters with MUSE . . . . . . . . . . . . . 23

1.5. Aims of this work . . . . . . . . . . . . . . . . . . . . . . 26

2. Publications 29

B. Giesers, S. Dreizler, T.-O. Husser, S. Kamann, G. Anglada Escudé, J. Brinchmann, C. M. Carollo, M. M. Roth, P. M. Weilbacher, and L. Wisotzki. A detached stellarmass black hole candidate in the globular cluster NGC 3201. In Monthly Notices of the Royal Astronomical Society, volume 475, pages L15-L19, March 2018. . . . . . 31

B. Giesers, S. Kamann, S. Dreizler, T.-O. Husser, A. Askar, F. Göttgens, J. Brinchmann, M. Latour, P. M. Weilbacher, M. Wendt, and M. M. Roth. A stellar census in globular clusters with MUSE: Binaries in NGC 3201. In Astronomy \& Astrophysics, volume 632, pages A3, December 2019. . . . . . . . . . . . . . . . . . . .

S. Kamann, B. Giesers., N. Bastian, J. Brinchmann, S. Dreizler, F. Göttgens, T.-O. Husser, M. Latour, P. M. Weilbacher, and L. Wisotzki. The binary content of multiple populations in NGC 3201. In Astronomy \& Astrophysics, volume 635, pages A65, March 2020.

3. Conclusions \& Outlook 63

3.1. A new statistical method . . . . . . . . . . . . . . . . . . . . . . 63 
3.2. The binary fraction of NGC $3201 \ldots \ldots \ldots \ldots$. . . . . . . . . . . 64

3.3. Orbital parameters of 95 binaries . . . . . . . . . . . . . . . . 64

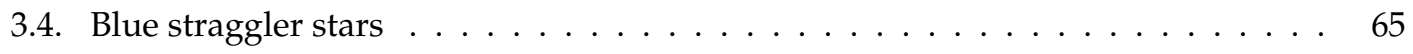

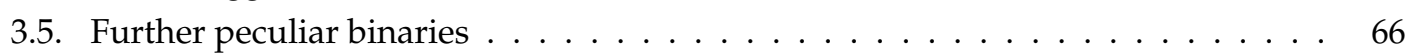

3.6. Black holes . . . . . . . . . . . . . . . . . . . . . . 66

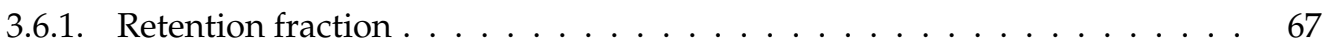

3.6.2. The search for an IMBH $\ldots \ldots \ldots$. . . . . . . . . . . . . . 68

3.7. Binaries in multiple populations $\ldots \ldots \ldots \ldots \ldots \ldots \ldots$

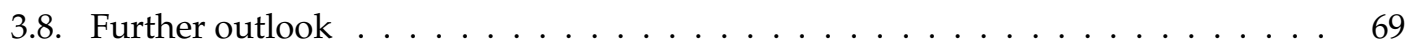

$\begin{array}{ll}\text { A. Appendix } & \mathbf{7 1}\end{array}$

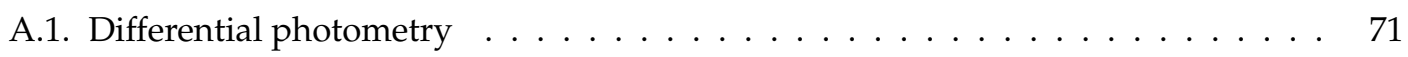

A.2. Simulation: Globular cluster model of radial velocities _ . . . . . . . . . . . 74

A.3. Binary fractions in the MUSE globular cluster sample . . . . . . . . . . . . 76

A.3.1. Expectation maximisation technique to identify binary stars . . . . . . 76

A.3.2. Sanity checks of expectation maximisation on simulations . . . . . . . 77

A.3.3. Results . . . . . . . . . . . . . . . . . 80

A.4. Matching of literature catalogues to MUSE data . . . . . . . . . . . . . . . . . . . . . . . . . . . . . 82

A.4.1. Matching method . . . . . . . . . . . . . . . . 83

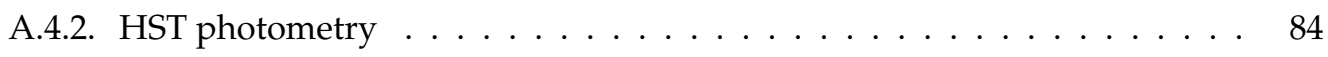

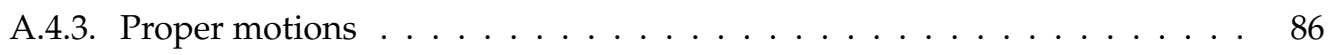

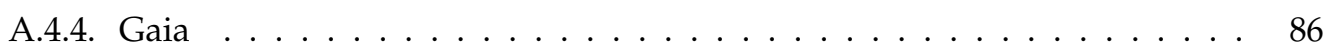

A.4.5. List of all matched catalogues . . . . . . . . . . . . . . . . . . . . . . 86

A.5. Stellar rotation of blue straggler stars . . . . . . . . . . . . . . . . . . 93

A.6. A systematic way to normalise the continuum in a spectrum . . . . . . . . . . 93

$\begin{array}{lr}\text { Bibliography } & 97\end{array}$ 


\section{List of additional Figures}

1.1. A colour image of the globular cluster $\omega$ Centauri (NGC 5139). . . . . . . . . . 3

1.2. Surface brightness profiles of the different globular clusters. . . . . . . . . . 5

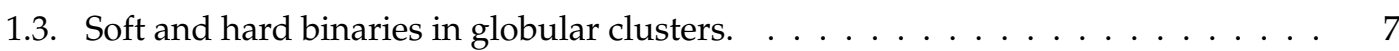

1.4. Correlation between binary fraction and absolute visual magnitude of Galactic

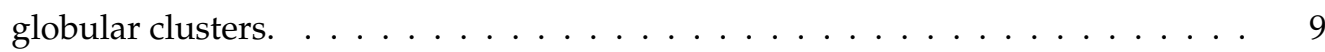

1.5. Example colour-magnitude diagram of NGC 3201. . . . . . . . . . . . . . . . . 11

1.6. Hierarchical multiple star systems. . . . . . . . . . . . . . . . . . . . 17

1.7. Radial velocity example curves. . . . . . . . . . . . . . . . . . . . . . 20

3.1. Globular clusters hosting black holes. . . . . . . . . . . . . . . . . . . . . 67

A.1. Example for uncertainties in differential photometry on MUSE data. . . . . . . . 72

A.2. Photometric variability in the CMD of NGC 3201. . . . . . . . . . . . . . . . 73

A.3. Simulated companion mass and period distributions of binaries in a globular

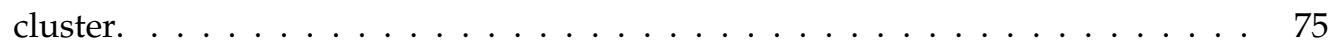

A.4. Probability density function of the single and binary star population. . . . . . . 77

A.5. The expectation maximisation method applied to different simulations showing

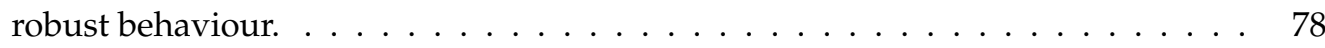

A.6. The expectation maximisation method applied to simulations with different epochs and different cluster densities. . . . . . . . . . . . . . . . . . . . . 78

A.7. The expectation maximisation method applied to simulations with different uncertainty factors. . . . . . . . . . . . . . . . . . . . 79

A.8. The correct and false-positive binary star detections for different epochs with the expectation maximisation method. . . . . . . . . . . . . . . . 80

A.9. Correlations of the binary fractions within the MUSE sample. . . . . . . . . . . 82

A.10.User interface to manage literature catalogues for the MUSE globular cluster

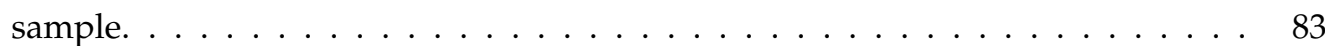

A.11.User interface to manage the VizieR tables of literature catalogues. . . . . . . . . 84

A.12.User interface to match the stars of a selected VizieR catalogue to the MUSE master catalogue. . . . . . . . . . . . . . . . . . . . 85

A.13.How to normalise a spectrum systematically. . . . . . . . . . . . . . . . . . . 94 


\section{List of additional Tables}

1.1. Parameters to describe the configuration of a binary system. . . . . . . . . . 21

1.2. The MUSE Galactic globular cluster GTO targets. . . . . . . . . . . . . . . . . . 24

A.1. Binary fractions in the MUSE globular cluster sample. . . . . . . . . . . . . . . 81

A.2. Literature catalogues which were matched to the MUSE master catalogue. . . . . 86 


\section{Acronyms}

47 Tuc 47 Tucanae (NGC 104)

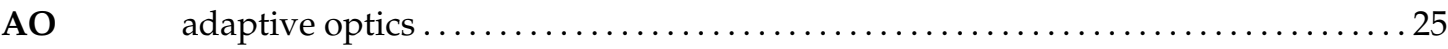

BH stellar-mass black hole

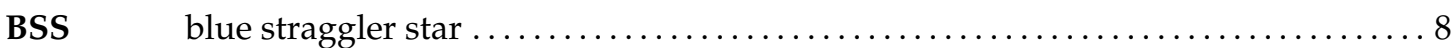

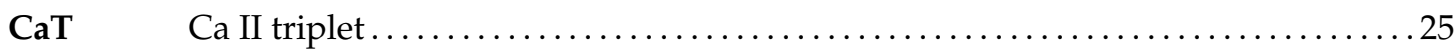

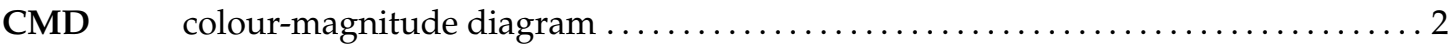

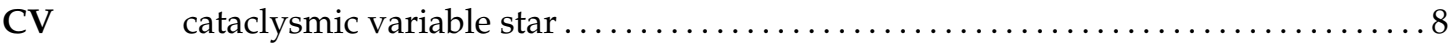

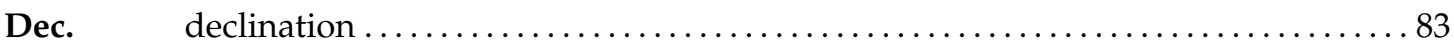

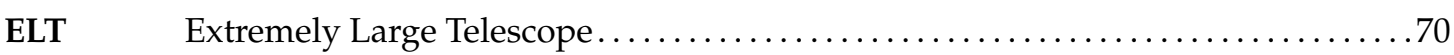

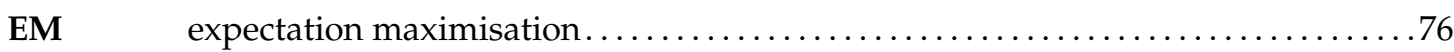

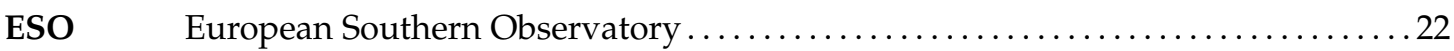

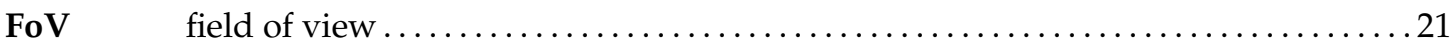

GALACSI Ground Atmospheric Layer Adaptive optiCs for Spectroscopic Imaging ........22

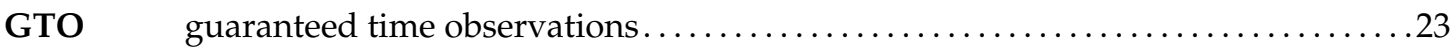

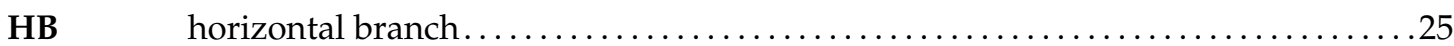

HRD Hertzsprung-Russel Diagram .................................. 1

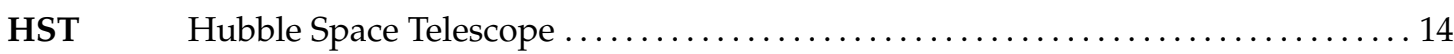

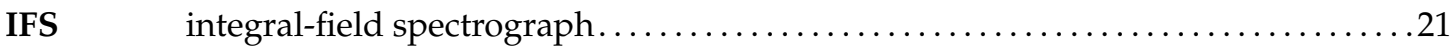

IMBH intermediate-mass black hole $\ldots \ldots \ldots \ldots \ldots \ldots \ldots \ldots \ldots \ldots \ldots \ldots \ldots \ldots \ldots$

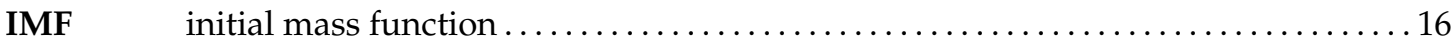

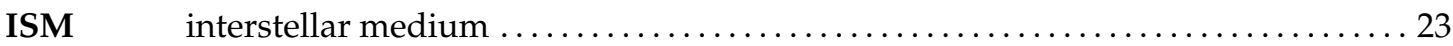

JWST James Webb Space Telescope................................. 70

KDE kernel density estimation ..................................... 77

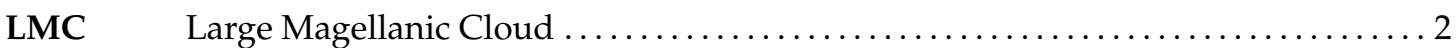

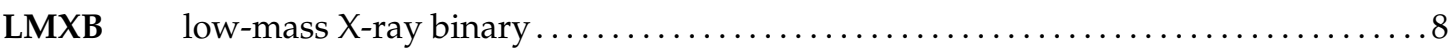

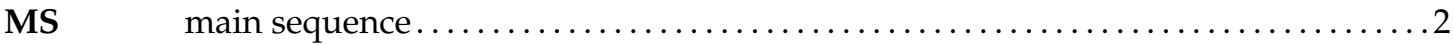

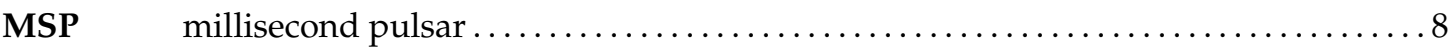




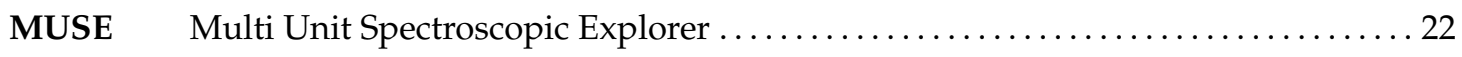

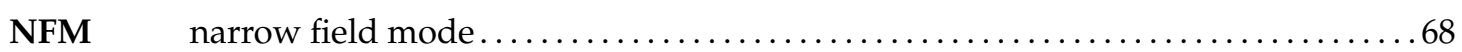

NGC New General Catalogue of Nebulae and Clusters of Stars

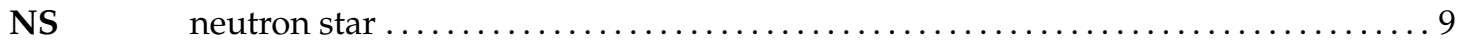

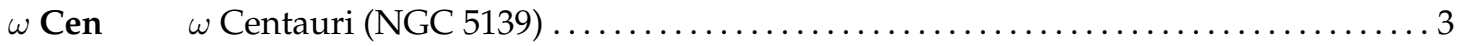

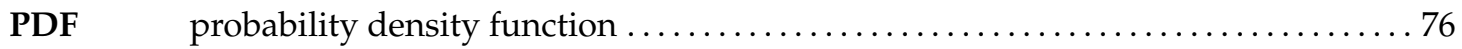

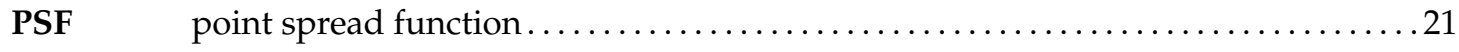

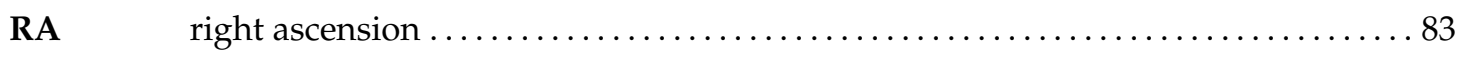

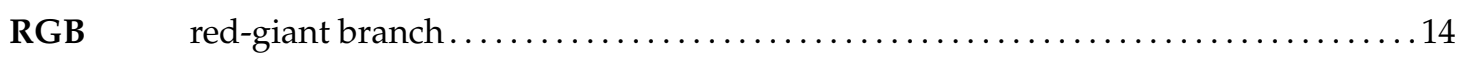

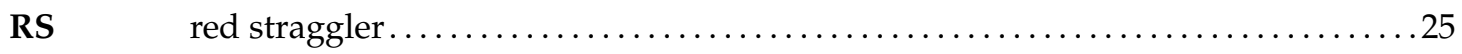

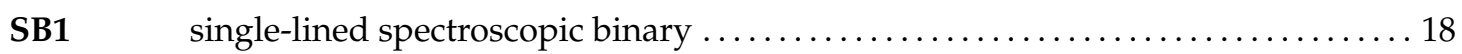

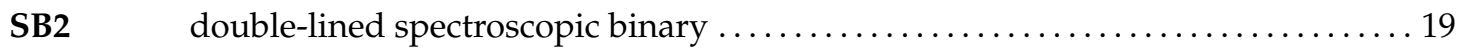

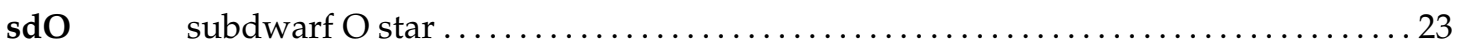

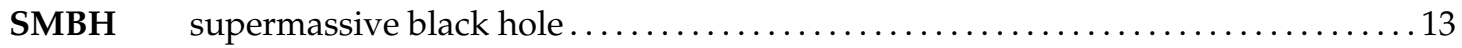

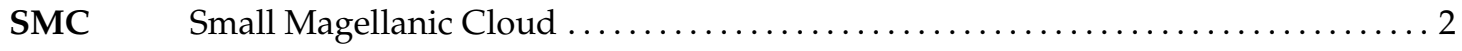

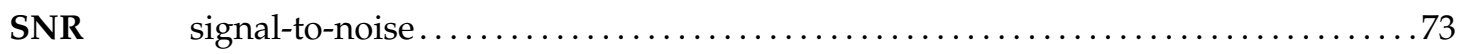

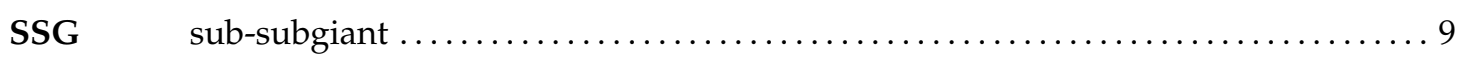

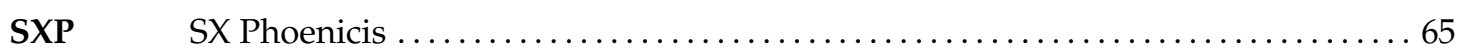

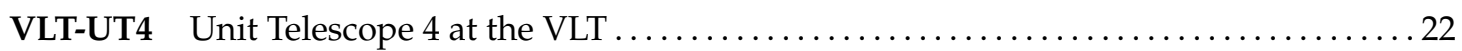

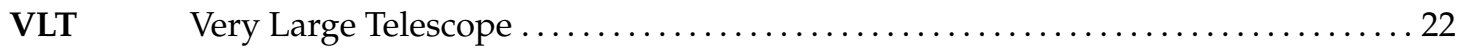

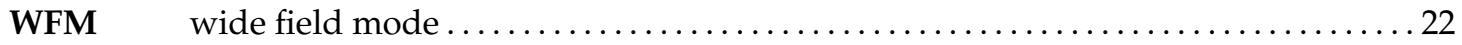




\section{Introduction}

In 1665, when the Galilean telescope was only almost 60 years old, the German amateur astronomer Johann Abraham Ihle observed Saturn in the constellation Sagittarius (Schultz, 1866). While he was observing, he recognised that one star close to Saturn did not look like the surrounding stars: the object appeared diffuse and nebulous, not like a point source (Kirch, 1682). A century later, telescopes had evolved and astronomers tried to make a name for themselves by finding comets. Comets are not stationary, compared to the fixed stars, and appear as diffuse objects on the night sky. The stationary objects, like the one Ihle discovered, looked similar to comets in the telescopes of that time and so often led to false discoveries of new comets. The french astronomer Charles Messier was annoyed by always finding the same stationary objects and created in the years from 1758 to 1784 a list of 103 stationary diffuse objects that are visible during the year from his site (Carroll \& Ostlie, 2017, p. 940). This Messier catalogue became famous as a collection of the brightest extended objects in the northern sky. We still give credit to Ihle for the first report of a globular cluster observation, later listed in the Messier catalogue as M22. Probably, it was Messier who was the first to resolve individual stars within the globular cluster M4.

A century later, photographic plates were introduced in astronomy and the era of modern astrophysics started. In 1897, the 40-in $(102 \mathrm{~cm})$ telescope at the Yerkes Observatory was commissioned, still today the largest refracting telescope ever used for astronomy (Carroll \& Ostlie, 2017, p. 155). In 1909 the German astronomer Karl Schwarzschild hired the talented Danish astronomer Ejnar Hertzsprung at the Göttingen university and they moved together shortly after to the Potsdam Observatory where the great refractor with $80 \mathrm{~cm}$ was commissioned in 1899 (Hearnshaw, 2014, pp. 129 ff.). At that time, the accepted theory for stellar evolution was that stars were born as hot, blue, and bright (early type) O stars, burn their mass and become cooler, red, and fainter (late type) M stars. Hertzsprung tried to find this correlation between absolute magnitude and spectral type. Parallel to Hertzsprung, Henry Norris Russel came to the same conclusions as Hertzsprung, that spectral type and luminosity of stars are correlated with each other (Carroll \& Ostlie, 2017, p. 220). But they both stumbled over bright, red stars which contradict the stellar evolution theory. They both independently introduced the term giant, to describe these luminous stars of late spectral type. Additionally, Russel used the term dwarf for faint stars of late spectral type. Bengt Strömgren later connected the two person names for the Hertzsprung-Russel Diagram (HRD), which shows the absolute magnitude in relation to stellar type of stars (Strömgren, 1933). He also suggested that star clusters should be used to understand stellar evolution better. 
The advantage of a star cluster is that member stars have roughly the same distance to the Sun. Hence, a comparison of their apparent magnitudes is sufficient. It also became clear that the spectral type of a star depends on its temperature and thus photometric colour (e.g. B-V or V-I). The elaborate HRD was simplified to the observational colour-magnitude diagram (CMD), also called observational HRD, which shows the apparent magnitude in relation to the colour of observed stars (e.g. Shapley, 1916, Greenstein, 1939, a modern example CMD is shown later in Fig. 1.5). With the crowded fields it took some time to get to the faint main sequence (MS) (e.g. Arp et al., 1952). The observers were at first surprised that the bright end of the main sequence seems to end where the giant branch starts, but this and other studies finally led to solving the stellar evolution puzzle.

From the distribution of globular clusters in the night sky Karl Bohlin concluded in 1909 that the Sun may not be at the centre of our Galaxy (Leverington, 1995). Distance measurements to RR Lyrae stars in globular clusters and with the help of parallaxes to the Andromeda Nebulae, Small Magellanic Cloud (SMC), and Large Magellanic Cloud (LMC) led to the Great ShapleyCurtis Debate about whether all spiral nebulae belong to the Galaxy or are external Galaxies. In the end, the debate was caused by false distance measurements: the distances to the globular clusters and thus the size of the Milky Way were overestimated, the distance to Andromeda, SMC, and LMC underestimated. Once the distance measurement problem was solved, the globular clusters were correctly used to estimate the size of the Milky Way.

Globular clusters can also be used for other astrophysical purposes. Reliable age estimates of globular clusters are a lower limit for the age of the universe. For cosmological models this is an important constraint (Freeman \& Bland-Hawthorn, 2002). In modern observations, globular clusters are extremely important test particles to determine the inner dynamics in distant galaxies (e.g. Trujillo et al., 2019).

\subsection{Globular clusters}

A star cluster is a group of stars that are gravitationally bound to each other. An open cluster is defined as a star cluster with hundreds up to 10000 stars. In the Milky Way, these clusters are normally tens of million of years young, since their gravitational potential is not sufficient to hold the members together for long times. In contrast, globular clusters with 10000 to several million stars (up to a mass of $5 \times 10^{6} \mathrm{M}_{\odot}$ for $\omega$ Centauri; NGC $5139=\omega$ Cen; see a modern colour image of $\omega$ Cen in Fig. 1.1), are spherically due to their deep gravitational potential (Meylan \& Heggie, 1997). This potential enables the globular cluster to survive within the potential of a galaxy for several Hubble times. Some globular clusters in the outer halo of the Milky Way are almost as old as the universe. The Milky Way hosts more than 150 globular clusters (Harris, 1996, 2010 edition) and they make up $2 \%$ of the light and baryonic mass of the Milky way halo (Freeman \& Bland-Hawthorn, 2002). On a large scale, the globular clusters are interesting probes of galaxy evolution and on small scales give insights into stellar evolution. It 


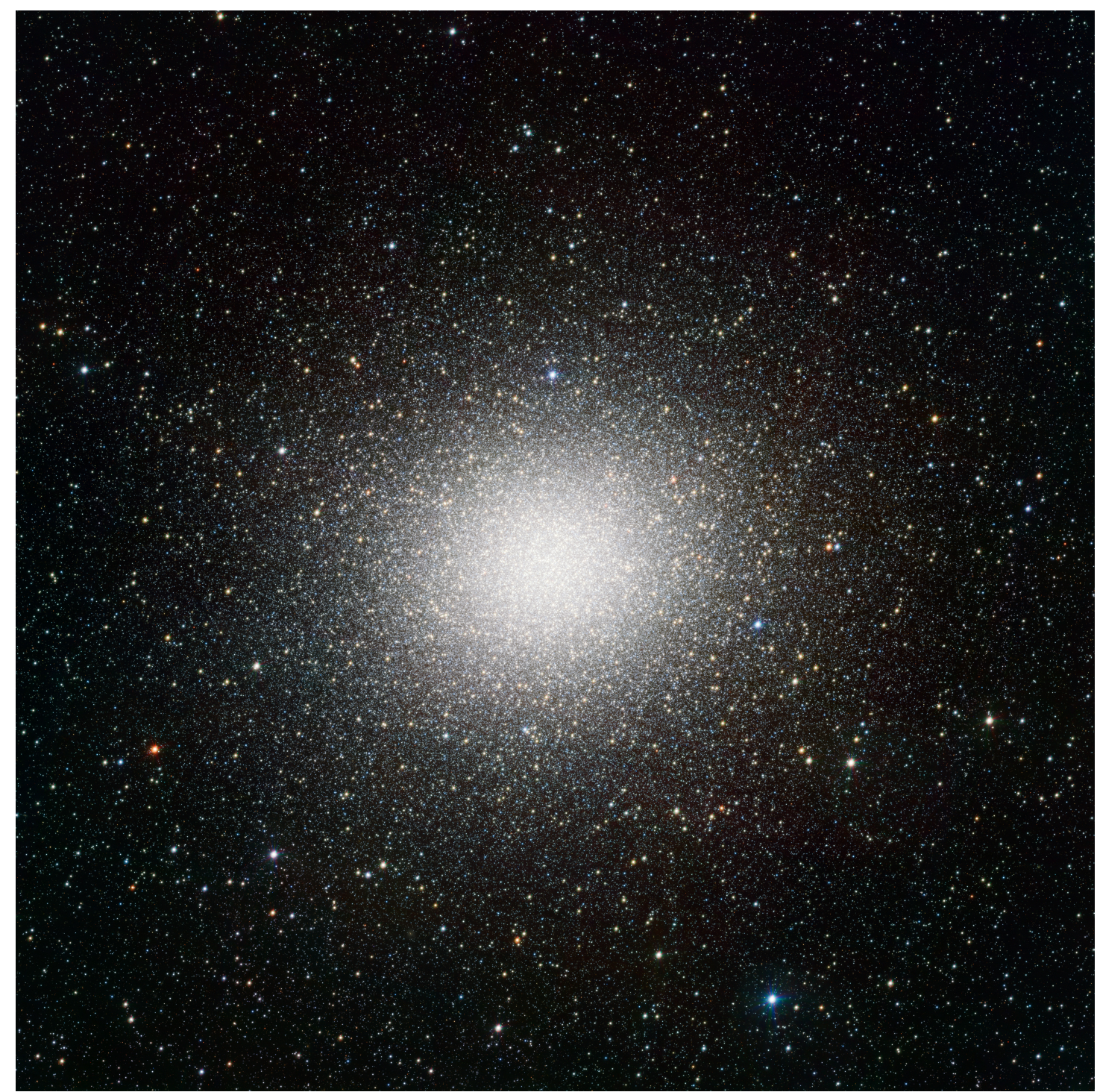

Figure 1.1.: A colour image of the globular cluster $\omega$ Centauri (NGC 5139) ( $\omega$ Cen). Credit: ESO/INAF-VST/OmegaCAM. Acknowledgement: A. Grado, L. Limatola/INAFCapodimonte Observatory.

was established in the 1950s that there are two populations of globular clusters in the Milky Way, the clusters with more metal-rich stars are concentrated in the Galactic disk, whereas the metal-poor clusters are located in the Galactic halo (Zinn, 1985). For a long time, it was thought that the properties of globular clusters are only determined by two parameters - the metallicity $[\mathrm{Fe} / \mathrm{H}]$ and a debated second parameter, predominantly cluster age (Dotter et al., 2010) - but since the discovery of multiple populations (for a review see Bastian \& Lardo, 2018) it is clear that globular clusters cannot be explained that easily. 


\subsubsection{Cluster formation}

The formation of globular clusters is still an unsolved puzzle but of great interest because globular clusters could be the key to understand galaxy formation. Most observed galaxies host a system of globular clusters, which suggests globular clusters are universal building blocks in the cosmos (Freeman \& Bland-Hawthorn, 2002). The early work by Searle \& Zinn (1978) supports the hypothesis that globular clusters could be protogalactic stellar fragments from which the Galaxy is formed (Freeman \& Bland-Hawthorn, 2002). In the early universe, the gas density was higher which resulted in a higher efficiency to form massive star clusters (e.g. Kruijssen, 2012). Therefore, most of the massive clusters formed very early, whereas today the formation of low-mass clusters is mainly observed. (Some very massive clusters still form today, e.g. Bastian et al., 2006). But how does the formation itself work? One idea is that there is nothing special about globular cluster formation (Pfeffer et al., 2018), but we are still lacking a consistent formation theory (e.g. Bastian \& Lardo, 2018) that can explain the observed multiple populations. More on multiple populations in Sect. 1.1.6.

Another scenario for globular cluster formation could be merger events of galaxies. During the merging of two galaxies shock fronts and matter overdensities form that could produce star clusters efficiently. A breakthrough is currently being achieved with the help of the Gaia mission (Gaia Collaboration et al., 2016): a recent paper constrains the origin of individual Galactic globular clusters to the Gaia-Enceladus galaxy (19\%), the Sagittarius dwarf galaxy (5\%), the Helmi streams (6\%), the Sequoia galaxy (5\%), and $40 \%$ formed in situ (Massari et al., 2019). This impressively reveals the merger and thus star formation events which built up the Galaxy. It would also mean, that the age of some globular clusters could be used to reconstruct the formation history of a galaxy (Ashman \& Zepf, 1992, Zepf \& Ashman, 1993, Massari et al., 2019). Furthermore, among the Galactic globular clusters, there could be six former nuclear star clusters (Kruijssen et al., 2019). One of these is $\omega$ Cen, which is probably the remnant of the nuclear star cluster of the accreted galaxy Gaia-Enceladus or the dwarf galaxy Sequoia (Ibata et al., 2019, Massari et al., 2019). Another example is M54 (NGC 6715), which comes from the Sagittarius dwarf galaxy.

\subsubsection{Cluster evolution}

Many globular clusters show high stellar densities of up to $10^{5} \mathrm{M}_{\odot} \mathrm{pc}^{-3}$, which lead to each member star having many gravitational encounters with other members during its lifetime. As a consequence, the initial orbital and thus velocity information of each star is lost over a specific time in a cluster, the so-called relaxation time. Of course, for single stars the relaxation time depends on the position and therefore on the stellar density at that position in the globular cluster. The core radius $r_{c}$ of a cluster is defined as the radius where the surface brightness drops to half its central value. Analogue to this, the half-mass radius $r_{h}$ is the radius where half of the cluster mass is included. For stars in the core of all Galactic globular clusters, the 


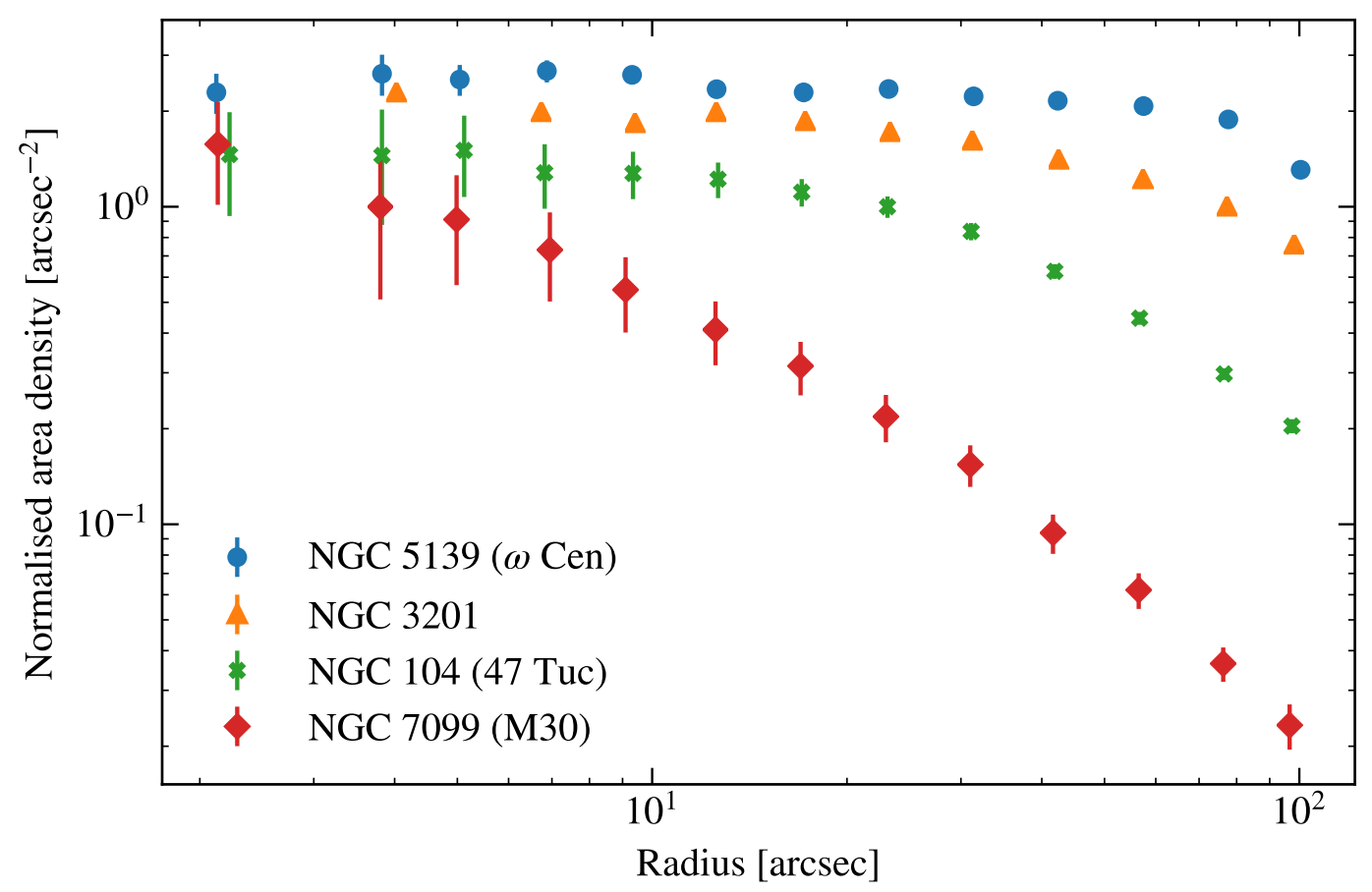

Figure 1.2.: Surface brightness profiles of the different globular clusters.

relaxation time is shorter than the cluster age ( $<10 \mathrm{Gyr}$, Harris, 1996). The same is true for the half-mass radius $r_{h}$ of most clusters and shows that most globular clusters become relaxed (forget their initial dynamical configuration) over their lifetime.

In thermodynamics, the velocities of particles with equal masses in a relaxed and closed system follow a Maxwell-Boltzmann distribution with the maximum determined by the thermodynamic temperature. In globular clusters, in terms of kinetic energy, it is exactly the same, the cluster wants to evolve to a Maxwellian distribution of stellar kinetic energies. This idea inspired the King (1966) model, which essentially assumes all stars have the same mass, resulting in a Maxwellian distribution of stellar velocities plus a cutoff energy where stars get unbound due to the galaxy potential. Because stars have different masses, an equilibrium distribution of kinetic energies can only be achieved if massive stars have lower velocities than less massive stars. In the gravitational potential of the cluster, when massive stars transfer kinetic energy to less massive stars, they sink a bit in the potential and transform potential energy to kinetic energy to become even faster. For the less massive stars it is the opposite: the gained kinetic energy is transferred into potential energy and they orbit slower on wider orbits around the cluster centre. This results in a mass segregation of stars along the radius to the cluster centre. King et al. (1995) observed this mass segregation in NGC 6397 and showed the underabundance of fainter sources in the cluster core. 
As a consequence of mass segregation, the core is loosing energy (heat) when less massive stars take the energy to larger radii. Due to tidal forces in the galactic potential, these stars in the outskirts are more likely to get stripped away. A study by Baumgardt \& Sollima (2017) has shown that, on average, a globular cluster looses $75 \%-90 \%$ of its initial mass. Thus, mass segregation causes a preferential removal of low-mass stars. This heat flow results in a gradual collapse of the core. That is why a globular cluster can be understood as a system with negative heat capacity: when the core heats up (core collapse), energy is released (Meylan \& Heggie, 1997). Due to their short half-mass relaxation times, old globular clusters should be core-collapsed (Gao et al., 1991). This contradicts the observations that less than $20 \%$ of all Galactic globular clusters are core-collapsed Harris (1996, 2010 edition). Figure 1.2 shows the surface brightness profiles of four selected globular clusters. Instead of brightness the number of stars in different radial bins divided by bin area and normalised to the number of stars in the bin at the cluster core radius was taken from the HST ACS globular cluster survey (Sarajedini et al., 2007, Anderson et al., 2008) for the different globular clusters. Two example globular clusters -47 Tucanae (NGC $104=47$ Tuc) and M30 - meet the simple cluster evolution expectation well and show a steep decrease in the surface brightness with larger radii. Following the definition for the core radius mentioned before, their cores are extremely small compared to the total cluster size and can be considered collapsed. The other two examples $-\omega$ Cen and NGC 3201 - show an almost constant profile. These clusters have a relatively large core radius compared to the total size and with respect to core collapse appear dynamically young. Hence, there must be some heat source to retard core-collapse on larger timescales in these and other globular clusters.

\subsubsection{Binaries}

One such heat source could be binary stars, but no detections of binaries in globular clusters were made until the late 1980s (McMillan et al., 1998). For example, the systematic spectroscopic study of Gunn \& Griffin (1979) found no binary systems. This suggested that globular clusters are born with few if not any so-called primordial binary systems. The few known Xray binaries, pulsars, and novae remnants in globular clusters were explained by dynamical capturing of stars during cluster evolution (Hut et al., 1992). Pryor et al. (1988) finally found one spectroscopic binary in the globular cluster M3 by observing 111 giant stars. Subsequently, more detections in several clusters were made and it became clear that binaries are crucial for understanding globular cluster evolution in general (Heggie, 1975, Goodman \& Hut, 1989, Hut et al., 1992).

Today we know that the total binary fractions in globular clusters are typically lower $(\lesssim 10 \%)$ than in the field (about $50 \%$ Duquennoy \& Mayor, 1991), but could easily reach values up to $20 \%$ in cluster centres due to mass segregation (binaries in clusters are in average more massive than the typical mass of a member star) (McMillan et al., 1998, Milone et al., 2012). Current observations show a typical increase of the globular cluster binary fraction by a factor 


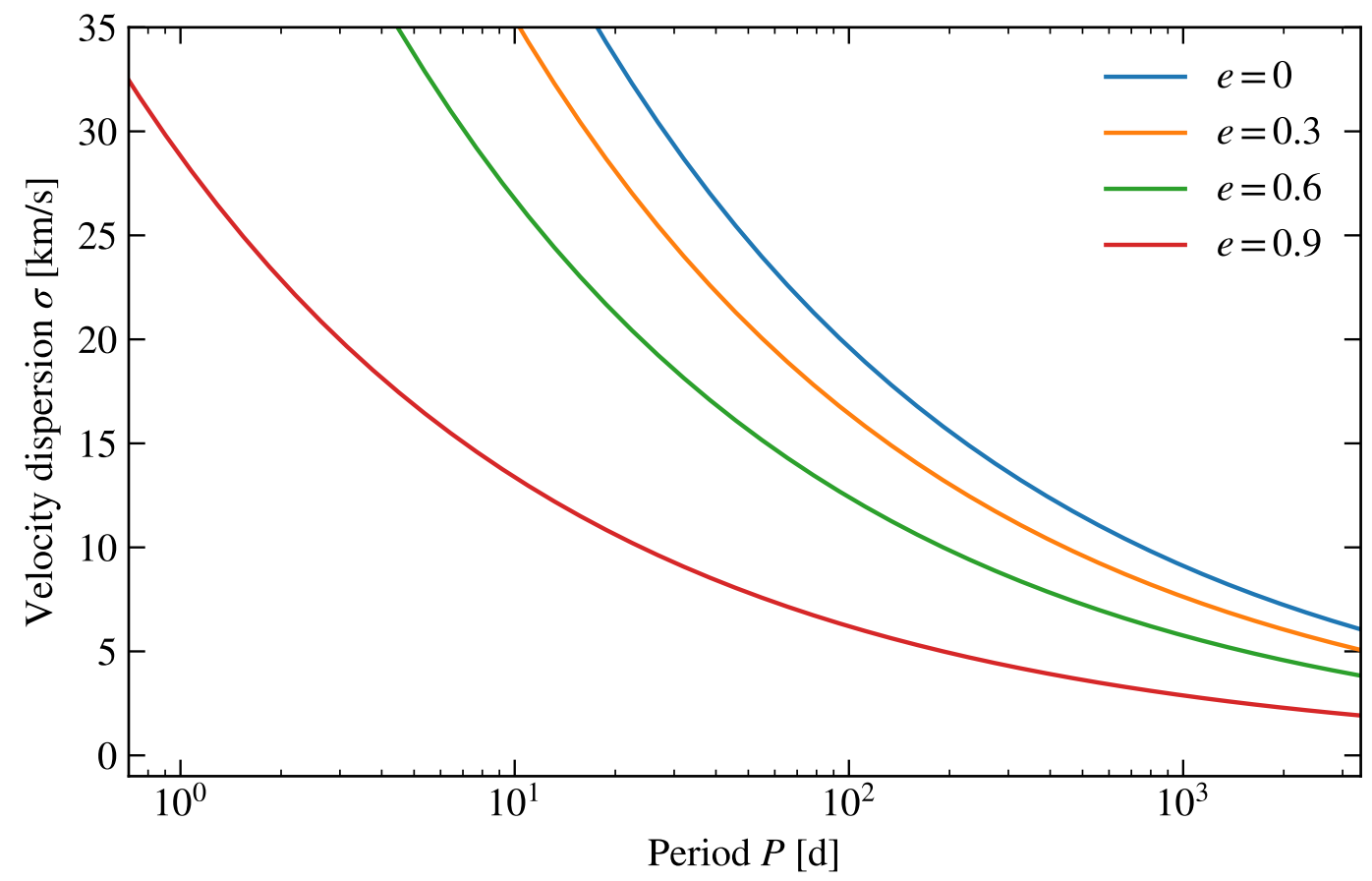

Figure 1.3.: Surrounding velocity dispersion as a function of the period of binary systems with hardness 1 and different eccentricities. Binaries below the curves are referred to as hard and above the curves as soft binaries.

of 2 from two core radius to the cluster centre (Milone et al., 2016). Thus, the evolution and dynamics of many cluster cores are controlled by binaries. We distinguish between hard (close) and soft (wide) binaries: hard binaries have binding energies exceeding the mean kinetic energy of cluster stars, whereas soft binaries are the opposite (McMillan et al., 1998). Figure 1.3 shows the threshold between hard and soft binary systems depending on the period of the binary system $^{1}$ for the velocity dispersion of stars surrounding the binary (Binney \& Tremaine, 2008, see also in this thesis the third publication in Chapter 2 for the used equations). For example, in the centre of $\omega$ Cen the velocity dispersion reaches values up to $20 \mathrm{~km} \mathrm{~s}^{-1}$ and a binary with a period of only $100 \mathrm{~d}$ is a soft binary and should not survive over the cluster lifetime. Through super-elastic dynamical scattering of stars with binary systems, hard binary systems normally get hardened by transferring energy to the encountering stars (from the hot system to the cooler star; Heggie, 1975). Thus, a large population of hard binaries acts as a heat source in their environment. This heat retards the core collapse (single stars can get on wider orbits again). In the picture of Fregeau et al. (2009), a globular cluster could be seen as a single self-gravitating system like a star, which burns its binaries like stars burn their hydrogen to prevent core collapse

${ }^{1} \mathrm{~A}$ binary system with a primary star mass of $0.8 \mathrm{M}_{\odot}$ and a companion mass of $0.6 \mathrm{M}_{\odot}$ was assumed. Constraints get even stronger if the masses differ more. 
(binary burning phase).

The destruction (ionisation) of binaries is not only possible by stellar encounters of (soft) binaries with single stars (binary-single encounters), but also by binary-binary encounters. During binary-binary encounters, one binary system could be hardened and the other system be resolved (Heggie, 1975, Fregeau et al., 2004). In the course of binary-single or binary-binary encounters, binary systems tend to exchange the less massive component with a more massive component. As a consequence, multiple binary exchanges equalise the mass components of a binary system over time. Of course, another destruction process of a binary system could be due to binary evolution, e.g. mass transfer, common envelope phase, stellar merger, supernovae, and so on.

For the creation of new binary systems by tidal capture in three-body systems (Fabian et al., 1975) an extremely high stellar density is necessary. Only some globular clusters reach high enough densities in their cores and the number of destruction events also increases with density (McMillan et al., 1998), so where does the current binary population in most clusters come from and why is it not a negligible binary fraction? The answer is primordial binaries: very high initial primordial binary fractions (about 50 to $100 \%$ ) are necessary to reproduce what we observe today (Ivanova et al., 2005, Leigh et al., 2015).

The mass segregation of binaries towards the cluster core and the destruction of these systems could reach an equilibrium and thus stabilise the core binary fraction over some time (Goodman \& Hut, 1989, Fregeau et al., 2009).

While binaries play an important role in the cluster evolution, the opposite is also true: the binary evolution itself is influenced (often accelerated) by the interactions of stars in the cluster. These interactions can lead to a number of extraordinary objects in globular clusters such as the following (Pooley \& Hut, 2006, Ivanova et al., 2008):

A cataclysmic variable star (CV) is a close binary system with a white dwarf accreting mass from a red dwarf, helium star, or subgiant. In globular clusters these systems are relatively faint and spectra of these objects are rare (Göttgens et al., 2019a).

A millisecond pulsar (MSP) is a pulsar with periods in the millisecond range. The pulsations originate from rapidly rotating neutron stars that have been recycled through accretion of matter from companion stars in close binary systems (Tauris \& van den Heuvel, 2006).

A low-mass X-ray binary (LMXB) is a compact binary system where one of the components is either a black hole or neutron star and the other component a less massive star filling its Roche lobe (Tauris \& van den Heuvel, 2006).

A blue straggler star (BSS) is a MS star in an open or globular cluster that is more luminous and bluer than stars at the main sequence turnoff of the cluster (see Sect. 1.1.4 for an introduction). 


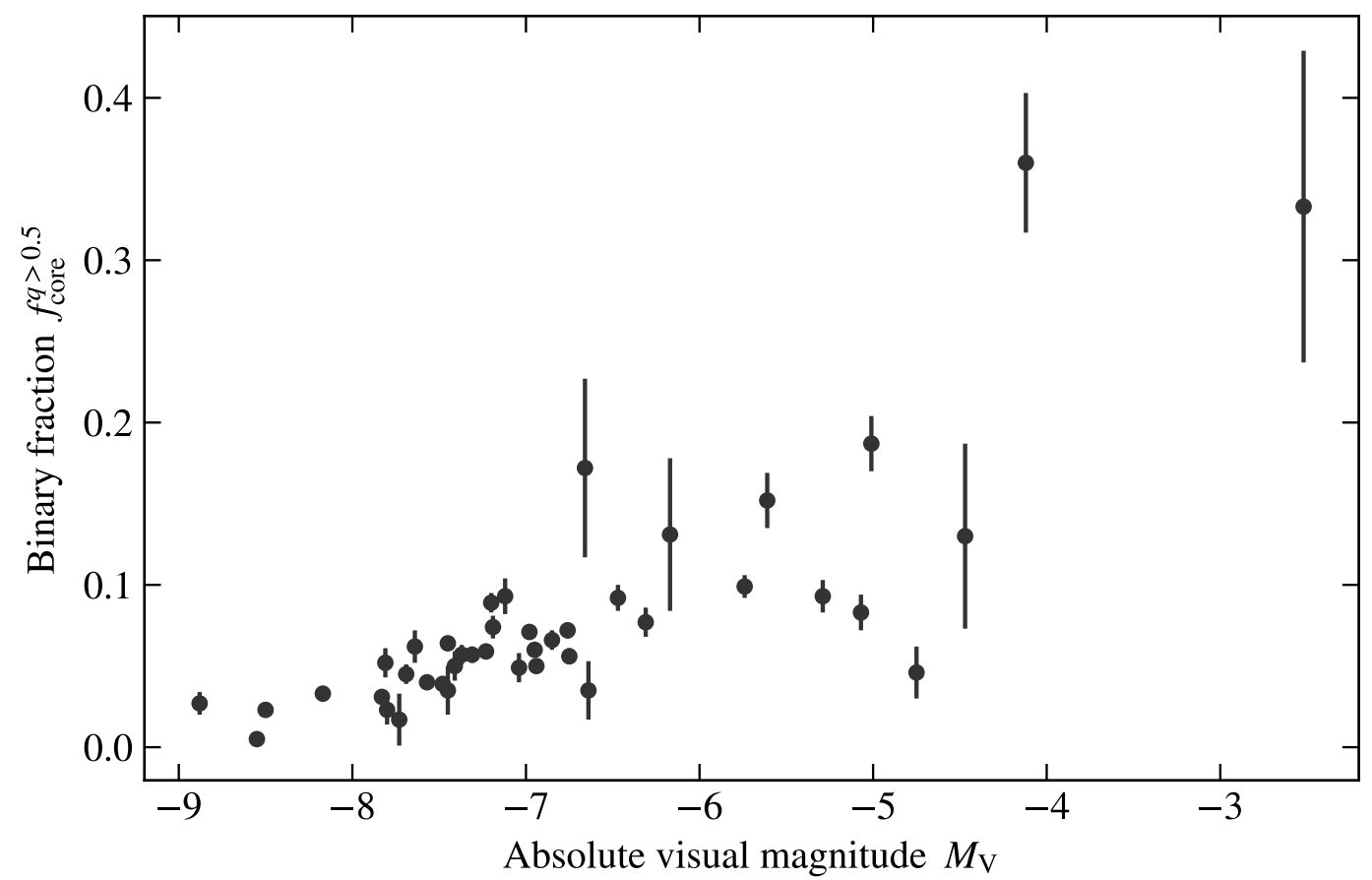

Figure 1.4.: Correlation between binary fraction and absolute visual magnitude of Galactic globular clusters.

A sub-subgiant (SSG) is a star located below the subgiant branch in the CMD of an open or globular cluster; see the second paper in Chapter 2 for a detailed description.

Stellar-mass black holes (BHs) can form binary systems just like stars and merge more easily by efficiently radiating gravitational waves; an introduction to BHs is given in Sect. 1.1.5.

The connections with binary evolution of BSSs, SSGs, and BHs are discussed in detail in this work.

An overabundance of LMXBs is observed in globular clusters and could be connected to the stellar encounter rate of clusters (Pooley et al., 2003). Related to this, an overabundance of neutron stars (NSs) and thus NS-NS or BH-NS binaries are likely (Ruiter et al., 2019). In contrast, CVs could be rare in clusters, since white dwarfs (in average less massive) are not mass segregated and due to binary exchanges less likely to be retained in binary systems. In general, the remnant fraction of compact or degenerated objects (e.g. NSs, BHs) in globular clusters is poorly known (Hansen \& Phinney, 1997), but binary studies could reveal these objects indirectly.

There are several observational techniques for identifying binaries; they are discussed in Sect. 1.2.1. One efficient technique is high precision photometry (Sollima et al., 2007a, Milone 
et al., 2012), which uses the fact that binary stars with both components contributing to the total brightness of one unresolved source have a position in the CMD that is different from single stars (e.g. at the MS often called binary main sequence, see Fig. 1.5). This technique has a high discovery efficiency for binaries with both components having similar masses, but it does not reveal the orbital parameters of the binary systems. Notwithstanding this, a significant anti-correlation between the binary fraction in a cluster and its absolute luminosity (mass) was found by Milone et al. (2012). Figure 1.4 shows this correlation between the binary fraction and the absolute visual magnitude of Galactic globular clusters based on the core binary fractions $f_{\text {core }}^{q>5}$ with binaries having a mass ratio $q>0.5$ published in Milone et al. (2012) and the absolute visual magnitudes published in Harris (1996, 2010 edition).

\subsubsection{Blue stragglers}

Sandage (1953) identified an extension of the main sequence towards bluer (hotter) and brighter stars in the CMD of the globular cluster M3 after carefully ensuring the cluster membership of this stellar population (comparable stars are visible in Fig. 1.5 of the globular cluster NGC 3201). At first this seemed to contradict the stellar evolution theory, since the same age of all stars should result in a CMD with a continuous transition from the main sequence to the giant branch (e.g. Williams, 1964). But McCrea (1964) and van den Heuvel (1967) had the idea that mass transfer in binary systems could explain all observed properties of these so-called blue straggler stars. It became clear that blue stragglers appear younger and more massive compared to the cluster population. In globular clusters only mass transfer in a binary system, binary mergers in binary-binary (binary-single) collisions, or the coalescence of two stars in a multiple-star system remain as plausible explanations for blue straggler formation (Stryker, 1993). But why are blue stragglers in globular clusters so interesting? First, blue stragglers are present in all observed clusters and are thus an integral component of globular clusters (Davies et al., 2004, Ferraro \& Lanzoni, 2009). Second, due to their relatively small number and their higher brightness (and blue colour), they are easier to observe. Third, the properties of blue stragglers link stellar evolution with cluster dynamics (Bailyn, 1995):

The last point is currently of great interest, since it not only allows us to understand the blue straggler formation, but also can be used to track the cluster evolution history. On the one hand, as described in Sect. 1.1.3, a globular cluster is formed with some amount of primordial binaries. Hard primordial binary systems could evolve to the mass transfer phase, which could end in the coalescence of both stars forming a "primordial blue straggler" (Hypki \& Giersz, 2017a). On the other hand, in high stellar density environments, blue stragglers could be formed from collisions of stars. For example, due to dynamical interactions with other stars or systems, wide binaries could become more and more eccentric until the binary components collide (Hypki \& Giersz, 2017a). A dynamically formed blue straggler could also result from a binary-binary encounter, in which two components collide and form a blue straggler (Hypki \& Giersz, 2017a). Interestingly, the largest portions of blue stragglers were found in NGC 288 and 


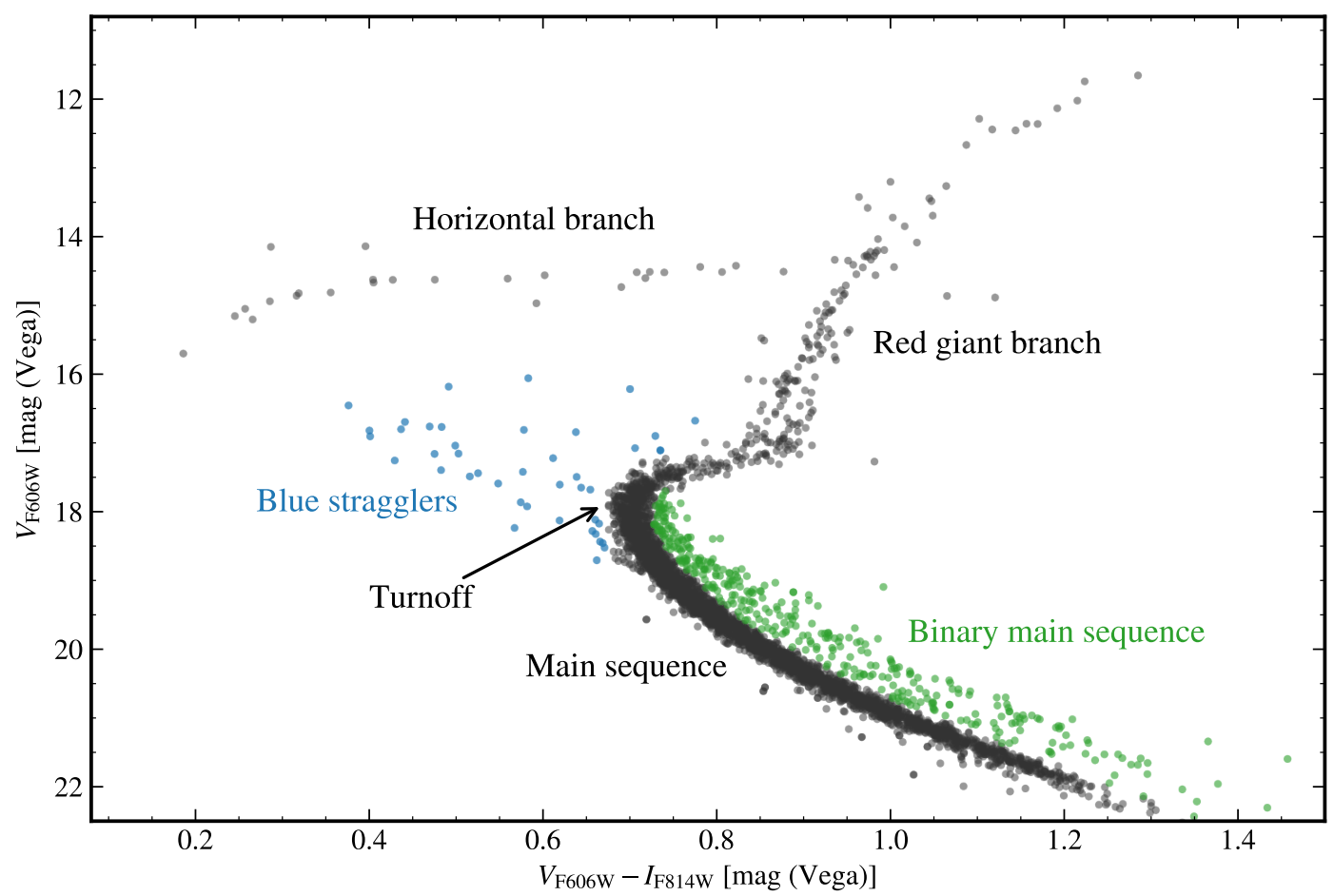

Figure 1.5.: Colour-magnitude diagram of the globular cluster NGC 3201 with annotations for important regions. Notice the photometric binary main sequence above the main sequence.

in M80, the former with the lowest and the letter with the highest central density, suggesting that both formation channels are efficient in producing blue stragglers (Davies et al., 2004, Ferraro \& Lanzoni, 2009). If only primordial blue stragglers would be present in globular clusters, their number should scale linearly with total cluster mass (assuming an equal primordial binary fraction in all clusters). If only dynamical blue stragglers would be present, their amount should be directly correlate with the collision rate of a given cluster. Actually, in the cores of globular clusters a correlation in the number of blue stragglers with the total core stellar mass was found by Knigge et al. (2009). Theoretically, denser globular clusters create more dynamical blue stragglers (Hypki \& Giersz, 2017a), whereas in low density clusters the evolutionary primordial blue stragglers should dominate (Beccari et al., 2013). Leigh et al. (2019) found that the blue straggler population of most clusters mainly consists of primordial blue stragglers, suggesting that the mass transfer scenario in binary systems is most efficient. In the post-core collapse clusters the dynamical collision scenario best explains the observation of the blue straggler population. This is also supported by a correlation between the core blue straggler fraction and the binary fraction of globular clusters (Sollima et al., 2008, Milone et al., 2012): the higher the binary fraction, the higher the probability that blue stragglers form. There are also globular 
clusters with two distinct blue straggler sequences, but it is not clear if they are connected to distinct formation channels or can be attributed to different formation events (e.g. Bailyn, 1992, Ferraro et al., 2009, Hypki \& Giersz, 2017b, Beccari et al., 2019).

We are still lacking a feasible observational discrimination between primordial and dynamical blue stragglers. In high resolution spectra a depletion of carbon and oxygen in a subpopulation of blue stragglers was found in 47 Tuc and M30 which could be explained by the mass transfer scenario (Ferraro et al., 2006, Lovisi et al., 2013b), but in other clusters that depletion was not found, which makes a correct interpretation more difficult (e.g. Lovisi et al., 2013a). Another way to get insights in the formation channel of blue stragglers could be the study of the stellar rotation of blue stragglers. Angular momentum could either be transferred via mass transfer to the blue straggler or gained in a merger event, but for a correct interpretation meaningful models and theories are currently missing (Simunovic \& Puzia, 2014, Mucciarelli et al., 2014, Leiner et al., 2018).

Another important discovery is that the spatial distribution of blue stragglers in globular clusters could be interpreted as a dynamical clock of the cluster (Ferraro et al., 2012, Alessandrini et al., 2016, Lanzoni et al., 2016). In the observations, the radial fraction of blue stragglers to other stars has at different cluster core radii one defined minimum in all globular clusters. The idea is that blue stragglers are sensitive test particles to mass segregation. In dynamically young clusters only a few blue stragglers from a relatively small influence sphere have migrated towards the centre, causing a minimum of the blue straggler frequency near the centre. In dynamical older clusters, this minimum is on larger cluster core radii, indicating mass segregation has affected a larger portion of the cluster. A clear correlation of this dynamical clock was found with the cluster relaxation time, enabling easy observations of blue stragglers to determine the dynamical age of a globular cluster. Properties that could influence the dynamical clock are the clusters binary fraction and the retention fraction of dark remnants in the core (Alessandrini et al., 2016).

\subsubsection{Black holes}

When non-rotating baryonic matter reaches a density higher than (Scheck, 2006)

$$
\rho=\frac{3 c^{6}}{32 \pi G^{3} M^{2}}
$$

- with the speed of light $c$, the gravitational constant $G$, and the mass of the matter $M$ - it has a so-called event horizon congruent to the Schwarzschild radius $r_{\mathrm{S}}$ at which the space time is curved in such a way that even light cannot escape the inner 'volume' (Schwarzschild, 1916, Carroll \& Ostlie, 2017, p. 635)

$$
r_{\mathrm{S}}=\frac{2 G M}{c^{2}} \approx M \times 2.95 \mathrm{~km} \mathrm{M}_{\odot}^{-1} .
$$


Because of this effect, these objects are called black holes (BHs). The interiors of BHs are not known and not accessible for experiments: Einstein predicted a singularity for these objects in his General Relativity, but such should not exist in nature. He also predicted gravitational waves (GWs) in space-time, created by accelerated masses and thought these tiny waves would never be detected, but those detected from a binary black hole merger by Abbott et al. (2016) directly proved multiple facts: GWs are existent, stellar-mass black hole mergers happen, and the GWs from the merger are completely consistent with General Relativity. Since then, multiple GW events have been observed and merger rates for our local universe have been estimated. At first, these merger rates were too high compared to most theories available, but it has been since shown that such high rates can be reproduced assuming a significant fraction (if not most) of black hole binaries are created in globular clusters (see the review by Rodriguez et al., 2018).

Currently two types of black holes are known in the universe, stellar-mass black holes (BHs) and supermassive black holes (SMBHs). Stellar-mass BHs are usually remnants of stellar evolution reaching the density described above and having masses up to few $100 \mathrm{M}_{\odot}$. At the end of stellar evolution, fusion processes come to a halt, causing the radiation pressure to decrease and the star to collapse into a degenerated (compact and faint) star. Stars remaining under the Chandrasekhar limit of about $1.46 \mathrm{M}_{\odot}$ become white dwarfs. Stars exceeding the TolmanOppenheimer-Volkoff limit of about $3 \mathrm{M}_{\odot}$ should collapse into black holes (Bombaci, 1996). Neutron stars (NSs) live in between these mass limits. In addition to the stellar evolution path, the creation of primordial BHs from pure hydrogen and helium is also conceivable (Carr, 1975). Stellar-mass black holes have been identified in X-ray compact binary systems and are now responsible for nearly all observed GW events to date. Theorists have long predicted that globular clusters could produce many BHs, but could only hold one or two of them due to natal kicks or via mutual interactions (e.g. Spitzer, 1969, Kulkarni et al., 1993, Sigurdsson \& Hernquist, 1993): BHs migrate towards the cluster centre due to the short relaxation time and kick each other out. However, observational evidence in the last years of BHs in globular clusters has proven that this idea is wrong (Maccarone et al., 2007, Strader et al., 2012, Chomiuk et al., 2013, Minniti et al., 2015). My dissertation makes a significant contribution to this by the discovery of a detached stellar-mass BH in a globular cluster (see the first paper in Chapter 2) which proves that BHs are not exceptional objects but represent an important ingredient of globular clusters. Thanks to these observations, more realistic simulations including binary evolution have been conducted, reproducing globular clusters with extended black hole systems (e.g. Mackey et al., 2007, 2008b, Morscher et al., 2015, Wang et al., 2016a, Kremer et al., 2018b, Askar et al., 2018). They show that $10 \%$ of the initial cluster mass is converted into BHs and that these BHs accumulate around cluster centres due to mass segregation. The retention fraction of these BHs, their mass spectrum, and the shape of the BH system within a cluster are still unclear (Belczynski et al., 2006, Baumgardt \& Sollima, 2017, Giesler et al., 2017). This effect is also important in the understanding of the number of GW sources created by globular clusters. If black holes are retained in the cores of globular clusters, they alter the cluster evolution completely: analogue to the binary 
burning in the core of a globular cluster (see Sect. 1.1.3), a black hole burning is also conceivable (Kremer et al., 2020). This thesis contributes to answering these open questions.

SMBHs are those indirectly observed in the centres of galaxies. A prominent example is Sagittarius $A^{*}$, a SMBH within the centre of the Milky Way. Stars around that SMBH have been used to weight the black hole and to detect the gravitational redshift caused by general relativity (Gravity Collaboration et al., 2018). Recently, the first direct image of the M87 SMBH has been published by the Event Horizon Telescope Collaboration et al. (2019), confirming the observation to be consistent with General Relativity. From observations of the early universe (high redshift) there is evidence for SMBHs formed rapidly from primordial gas clouds (e.g. Volonteri, 2012, Wise et al., 2019). Another scenario to form a SMBH is the runaway merging of massive stars or stellar-mass black holes in globular clusters, an effect that strongly depends on the cluster metallicity (Gieles et al., 2018). Scaling relations linking black hole masses to galaxy bulge masses and velocity dispersion have been found (McConnell \& Ma, 2013): the lower the galaxy mass, the lower the mass of the central BH, although no observational evidence for SMBHs below $10^{5} \mathrm{M}_{\odot}$ in low mass galaxies $\left(<10^{10} \mathrm{M}_{\odot}\right)$ is present (Kormendy \& Ho, 2013).

It is natural to search for BHs in globular clusters: following the scaling relation, they could have central BHs with masses between the known stellar-mass BHs and SMBHs. These BHs are therefore called intermediate-mass black holes (IMBHs). To date, there is no convincing observational evidence for these black holes (Baumgardt, 2017, Tremou et al., 2018). For example, Lützgendorf et al. (2011a) claimed to have found an IMBH in NGC 6388 but this discovery was justifiably questioned by Lanzoni et al. (2013). The observational bias comes from the fact that stellar-mass black holes and binaries can influence the kinematics of surrounding stars in the same way as an IMBH would do (e.g. Mann et al., 2019). This is where our MUSE survey (see Sect. 1.4.2) comes in, helping to solve this puzzle. Currently, $\omega$ Cen and M54 are promising candidates to host an IMBH, because they could be former nuclei of galaxies as mentioned before in Sect. 1.1.1. Furthermore, IMBHs could be the seeds for SMBHs in the early universe, and IMBHs could be built up from BHs. The most likely place in which these formation scenarios are possible are globular clusters. Finally, GW observations could also help to solve this puzzle.

\subsubsection{Multiple populations}

Until 1999 it was considered that all stars in globular clusters, except some peculiar objects, are roughly formed at the same time from the same material, hence are a simple single stellar population. The discovery of two distinct stellar populations in $\omega$ Cen by Lee et al. (1999) questioned this assumption. Of course, as mentioned in Sect. 1.1.1, $\omega$ Cen is not a typical globular cluster, but with the spectroscopic discovery of the sodium-oxygen, magnesium-aluminium, carbon-nitrogen anti-correlations, and helium-enriched stellar populations in several globular clusters, it became clear that multiple populations are present in most, probably all massive Galactic globular clusters (e.g. Kraft, 1994, Gratton et al., 2001, 2004, Piotto et al., 2005, Carretta et al., 2007a, 2009b). With recent Hubble Space Telescope (HST) photometry, often the red-giant 
branch (RGB) and the MS show multiple stellar sequences (e.g. Bedin et al., 2004, Piotto et al., 2007, Milone et al., 2008, Piotto et al., 2015, Milone et al., 2017). Extending our view to other galaxies like the SMC and LMC revealed that extragalactic globular clusters also host multiple stellar populations (e.g. Mackey et al., 2008a), but some intermediate-age globular clusters seem not to exhibit multiple populations (Li et al., 2014, de Grijs \& Li, 2018). In summary, multiple populations occur in clusters more massive than about $10^{4} \mathrm{M}_{\odot}$ and older than $2 \mathrm{Gyr}$ (Martocchia et al., 2018).

No generally accepted formation scenario is known for the variety of multiple populations in globular clusters (see Bastian \& Lardo, 2018, for a review). The kinematic properties of stars in the different populations in connection with their chemical abundances could give some clues about the formation of the populations. In most Galactic globular clusters, two different populations are apparent: the more primordial population 1 (P1, in the past called first generation) and the enriched population 2 ( $\mathrm{P} 2$, in the past called second generation). The relative number of P1 and P2 stars depends on the mass of the globular cluster: in high mass globular clusters the P1 stars are outnumbered by the P2 stars. Additionally, the P2 population could again show further sub-populations as in NGC 2808 (Piotto et al., 2007, Milone et al., 2015). The P2 stars are normally more centrally concentrated than the P1 stars (e.g. Sollima et al., 2007b, Lardo et al., 2011, Kučinskas et al., 2014). There are also exceptions to this: for example, P1 stars in M15 are more concentrated than P2 stars in the centre and also in the outskirts (Larsen et al., 2015). In young globular clusters, a difference in stellar spins between populations could mimic the appearance of chemically different populations (Kamann et al., 2018a). However, a fast-rotating star has a slightly different hydrostatic equilibrium than a slow-rotating star which could alter its stellar evolution (de Grijs \& Li, 2018). Another misidentification of populations could be due to the binary main sequence as described in Sect. 1.1.3 (for a deeper discussion see also the third paper in Chapter 2). Findings of lower velocity dispersions in P2 populations compared to the corresponding P1 populations can also be attributed to binary stars, increasing the dispersion in P1 (Dalessandro et al., 2018).

As discussed in Sect. 1.1.3 the binary fraction originates from the primordial binary fraction in most globular clusters, thus most binaries can be considered as primordial. The dominating destruction process of these binaries depends on the cluster density. Hence, the binary fraction of a more centrally concentrated population should decrease over time compared to an outer population. The lower binary fraction of P2 stars compared to P1 stars has been confirmed by observations (e.g. D'Orazi et al., 2010, Lucatello et al., 2015, Dalessandro et al., 2018) and can also be modelled (e.g. Vesperini et al., 2010, Hong et al., 2015). Lucatello et al. (2015) derived a P1 binary fraction of $(4.9 \pm 1.3) \%$ and a P2 binary fraction of $(1.2 \pm 0.4) \%$ from 21 binaries in ten globular clusters, and Dalessandro et al. (2018) found 12 binaries with a P1 binary fraction of about $14 \%$ and a P2 binary fraction of lower than $1 \%$ in NGC 6362. The mixed populations in the cores of globular clusters experience the same destruction processes over time and should end up with similar binary fractions (Hong et al., 2015, 2016). One problem is that the simula- 
tions use equal masses for all stars. There are two possible explanations for the conservation of different population binary fractions in the core: if on average P2 stars have more radial orbits in the cluster potential than P1 stars, most of the time the P2 stars seem to be on wider orbits but experience the dense core once per revolution, thus decreasing the binary fraction of P2 more in contrast to P1. Another explanation would be different primordial binary fractions of the populations, but apart from a slightly different chemical composition, no complete theory exists to explain the big differences in binary fraction we observe (Dalessandro et al., 2018).

Beyond the internal cluster formation scenarios, some cluster populations could be attributed to merger events: for example $\omega$ Cen and NGC 1851 show some odd populations that could have been accreted (Lee et al., 1999, Carretta et al., 2010).

\subsubsection{Simulations}

A typical Galactic globular cluster was born with a million stars and evolves over at least $10 \mathrm{Gyr}$. Thus, the simulation of the gravitational interactions between stars, the stellar evolution, binary interactions, natal kicks of NSs and BHs, and influence of an external gravitational potential of a galaxy is a computational challenge. A direct approach would be a so-called $N$-body integrator, which calculates the gravitational forces between all $N$ stars on every dynamical time step. An indirect approach would be a Monte Carlo method which approximates these forces. Additionally, both approaches need models for stellar evolution, binary evolution, and a galaxy potential. These additional models could actually be identical for both approaches. Such simulations usually start with a defined number of already formed stars drawn from an initial mass function (IMF) such as the Salpeter (1955) IMF or Kroupa (2001) IMF. The spatial distribution of these stars could be set up using a King (1966) model or observations of young massive clusters. Assumptions, such as dynamic equilibrium, homogeneous mass distribution, and no substructures in the initial configuration of a simulation, should be kept in mind when comparing with observations (de Grijs, 2010).

A lot of progress has been made simulating realistic globular clusters. On the $N$-body side, in 20 years the number of stars simulated grew from $10^{3}$ (Spurzem \& Aarseth, 1996) and $10^{5}$ (Baumgardt \& Makino, 2003) to $10^{6}$ (Wang et al., 2016a) recently. These $N$-body simulations are essential to validate the Monte Carlo methods. Actually, Monte Carlo methods have produced large globular cluster simulations earlier (e.g. $10^{6}$ stars in Giersz, 2006), but only the comparison with the $N$-body simulations gives good confidence to them. Ones a Monte Carlo code is validated, the parameter space can be investigated on much shorter timescales compared with $N$-body simulations. Several Monte Carlo codes have been introduced (e.g. Spurzem \& Giersz, 1996, Giersz, 1998). Based on the Monte Carlo approach of Hénon (1971) over the years an advanced simulation called MOCCA has been developed and validated against $N$-body simulations (Hypki \& Giersz, 2013, Giersz et al., 2013, Leigh et al., 2013, 2015). With this code a survey of simulated models has been created (e.g. Askar et al., 2017, 2018, Arca Sedda et al., 2018) which represents most of the Galactic globular clusters. 


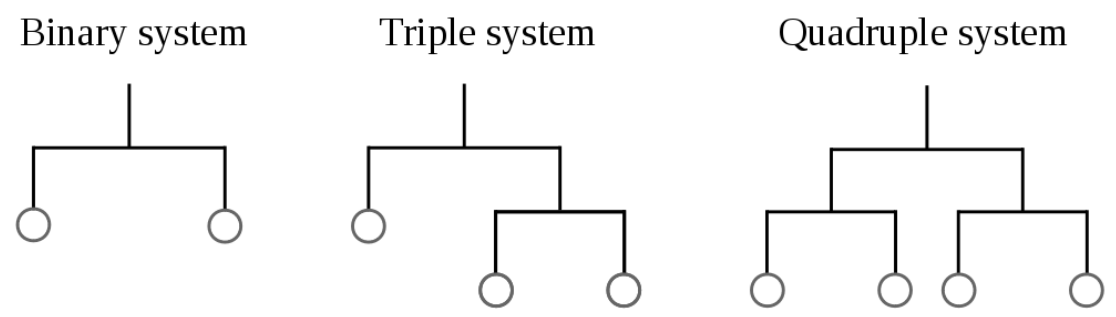

Figure 1.6.: Schemes for hierarchical multiple star systems. Every level connects two components in a quasi binary system. Circles represent stars.

Modern $N$-body simulations such as the DRAGON simulation and Monte Carlo simulations, such as the MOCCA simulation, also include the dynamical evolution of binary systems. Because it is way more computationally expensive to include binary evolution into $N$-body simulations, the initial (primordial) binary fraction is usually low, e.g. $5 \%$ in the DRAGON simulation. In the Monte Carlo simulations it is even possible to have a $100 \%$ binary fraction. Recent simulations (e.g. Hypki \& Giersz, 2013) are able to reproduce binary products such as blue straggler stars, cataclysmic variables, binary black holes, and so on. As explained in Sect. 1.1.3, it is more likely that high primordial binary fractions $>50 \%$ are needed to explain the observational properties of most globular clusters and the peculiar stars they contain, but there is still no final agreement within the literature. These modern simulations also retain in contrast to the simplified Spitzer (1969) model - many black holes and could be used to predict the number of gravitational wave events to expect from globular clusters. For example, the DRAGON simulation confirmed for the first time the existence of a BH subsystem in a full direct $N$-body simulation. More on the simulation results of black holes in Sect. 1.1.5.

\subsection{Multiple star systems and binaries}

A multiple star system is a system in which multiple stars are bound to each other by gravity. A famous example for such systems is Mizar, which with Galilean telescopes is easy to resolve into two components Mizar A and Mizar B. Until 1889 this system was thought to be a binary system, but in that year Mizar A was proved to be a spectroscopic binary (Pickering, 1890). In 1908 the same happened to Mizar B (Ludendorff, 1908, Frost, 1908). These four stars are a good example for a hierarchical quadruple star system. Since only two-body systems are stable over long timescales, and close stars act gravitationally almost as a single point mass on another distant star (or close binary), hierarchical systems like in Fig. 1.6 sketched live longer. 


\subsubsection{Observational techniques}

From an observational point of view, there are different ways to observe multiple star systems. Components of multiple star systems, such as Mizar A and B can be directly observed with telescopes if the angular separation of the components is higher than the minimal angular resolution $\theta_{\min }$ of the telescope

$$
\theta_{\min }=1.22 \frac{\lambda}{D}
$$

This is the well-known Rayleigh criterion for a circular aperture $D$ and the effective observational wavelength $\lambda$. Actually, with modern optical interferometry, even small angular separations, like the components of Mizar A have, can be directly resolved (Benson et al., 1997). For simplicity, we concentrate hereafter only on binary systems, since most multi-star systems should be hierarchical systems with two bodies being on the dominant timescale for the dynamics of the system. With astrometry the binary system could also be analysed if only one "wobbling" component could be measured.

Another indirect method to observe binaries is to look for flux changes of the system. In the case of an eclipsing binary system, a change in brightness is measurable when one component eclipses the other component. If the inclination is correct, such systems have a secondary eclipse in addition to the primary eclipse. With high precision photometry it is also possible to detect nearby binary systems which are not eclipsing due to changes in geometric effects, e.g. the reflection of light from one component onto the other, the deformation of one star due to the companion star, or relativistic beaming (lensing) of star light.

Exotic objects, like the ones described in Sect. 1.1.3, can also be identified by radio, $\mathrm{X}$-ray, or Gamma-ray searches, depending on the flux in these electromagnetic wavelengths.

With Doppler spectroscopy it is possible to observe the Doppler effect and thus the stellar velocity in line of sight in stellar spectra due to gravitational effects. The resolving power $R$ of a spectrograph

$$
R=\frac{\lambda}{\Delta \lambda}
$$

with the smallest resolvable difference in wavelength $\Delta \lambda$ at the wavelength $\lambda$ determines what changes are resolvable in a spectrum. In the case of a binary system with one component being magnitudes brighter than the other component, at any point in time, the spectrum would look the same except for a wavelength shift. This wavelength shift $\Delta \lambda$ could be translated to the so-called line of sight (LOS) non-relativistic radial velocity $v_{\mathrm{r}}$

$$
\frac{\Delta \lambda}{\lambda}=\frac{v_{\mathrm{r}}}{c}
$$

These binaries are called single-lined spectroscopic binaries (SB1), whereas binaries with both 
components visible in the spectrum are called double-lined spectroscopic binaries (SB2). The spectra of SB2 can look different at different times, since the Doppler shifted spectra of both stars are combined in one spectrum. For SB2 no absolute wavelength calibration is needed to derive the system properties, whereas for SB1 an accurate wavelength reference is needed to calculate $\Delta \lambda$ consistently at every time step.

\subsubsection{The radial velocity technique of binary systems}

To derive the Keplerian parameters from the radial velocities of a binary system, an orbital model is needed. For circular orbits of a two-body system, using Kepler's third law, the radial velocity semi-amplitude $K_{1}$ of the object $M_{1}$ is the projection of its orbital velocity $v_{1}$ with the orbital plane inclination $i$ :

$$
K_{1}=v_{1} \sin i=M_{2} \sqrt{\frac{G}{a\left(M_{1}+M_{2}\right)}} \sin i
$$

with the body masses $M_{1}$ and $M_{2}$, the semi-major axis $a$ of the system, and the gravitational constant $G$. Therefore, the time $t$ dependent radial velocity $v_{\mathrm{r}, 1}(t)$ is

$$
v_{\mathrm{r}, 1}(t)=v_{0}+K_{1} \cos M(t) \quad \text { with } M(t)=2 \pi\left(\frac{t}{P}+\phi\right)
$$

the so-called mean anomaly, the barycentric system velocity $v_{0}$, the period $P$, and the phase $\phi$ (since periastron) of the system.

For the general two-body problem with eccentricity $e$ the Kepler equation

$$
E(t)-e \sin E(t)=M(t)
$$

with the eccentric anomaly $E(t)$ is helpful. Note, this equation is not analytically solvable for $E(t)$, but using the Banach-Caccioppoli fixed-point theorem, it is possible to approximate the equation iteratively

$$
\begin{aligned}
E(t)_{0} & =M(t) \\
E(t)_{n+1} & =M(t)+e \sin E(t)_{n} .
\end{aligned}
$$

The true anomaly $T(t)$ can be expressed as (Lovis \& Fischer, 2010)

$$
T(t)=2 \arctan \left(\sqrt{\frac{1+e}{1-e}} \tan \frac{E(t)}{2}\right) .
$$




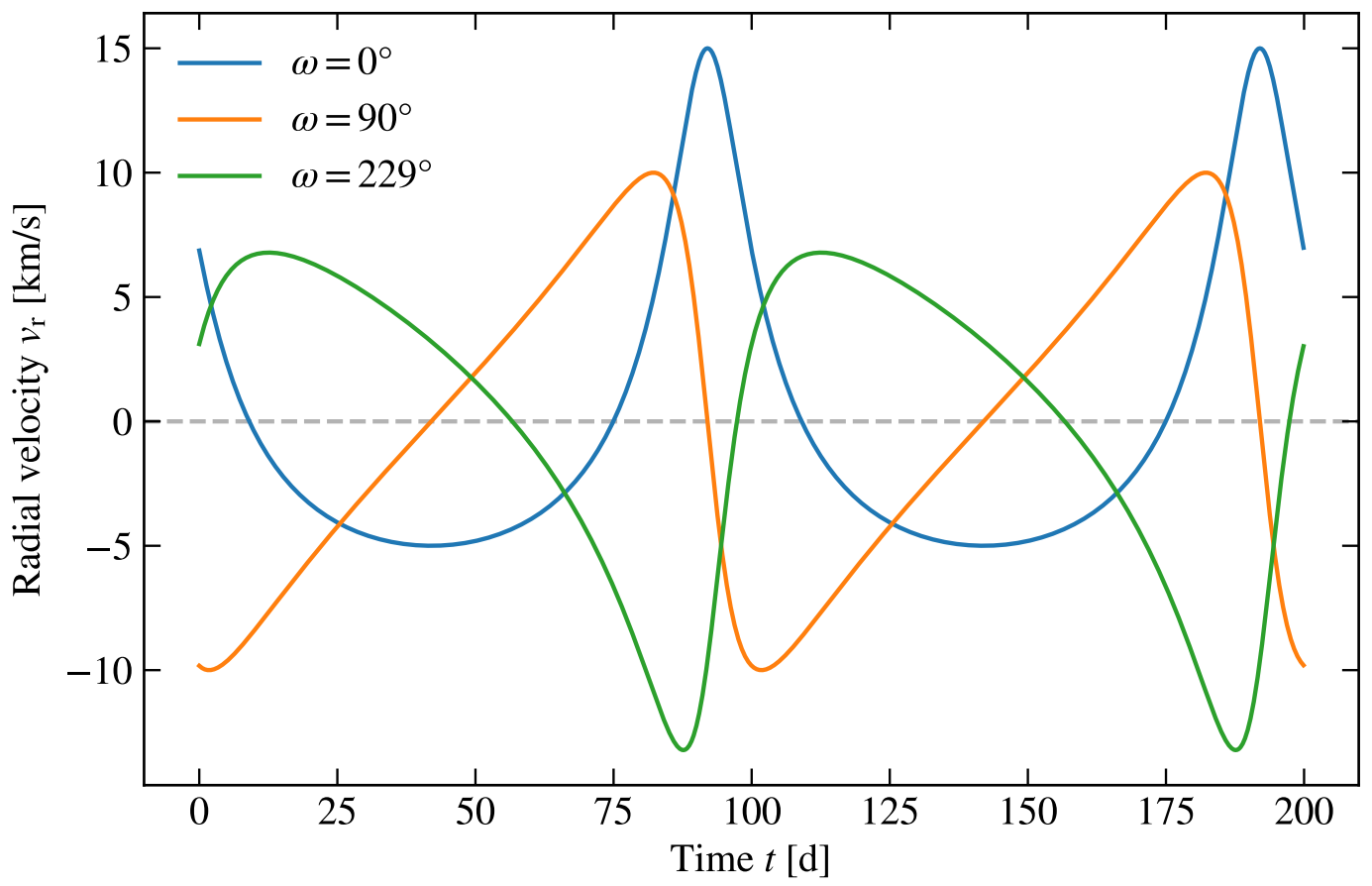

Figure 1.7.: The radial velocity curves of an example binary system with semi-amplitude $K_{1}=10 \mathrm{~km} \mathrm{~s}^{-1}$, period $P=100 \mathrm{~d}$, eccentricity $e=0.5$, phase $\phi=30^{\circ}$ and three different arguments of periapsis $\omega$ (see legend).

The radial velocity induced by the true anomaly of the object $M_{1}$ is finally

$$
v_{\mathrm{r}, 1}(t)=v_{0}+K_{1}[e \cos \omega+\cos (T(t)+\omega)] \quad \text { with } K_{1}=M_{2} \sqrt{\frac{G}{a\left(M_{1}+M_{2}\right)\left(1-e^{2}\right)}} \sin i
$$

the radial velocity semi-amplitude $K_{1}$, and the argument of periapsis $\omega$. For the case with $e=0$ these equations resemble Eq. 1.6 and Eq. 1.7. Figure 1.7 shows an eccentric binary system example with the used parameters given in the description of the figure. It demonstrates how the same orbit looks in radial velocities when only changing the argument of periapsis and thus the projection of the orbit to the observer. It also shows how asymmetric and different from sinusoidal functions a radial velocity curve can appear.

Using these equations, Kepler's third law, and sufficient radial velocity measurements of a SB1 binary system, the semi-amplitude $K$ and thus period $P$ and eccentricity $e$ can be calculated. From radial velocities it is not possible to determine the inclination $i$ of the system. Knowing one of the masses (e.g. $M_{1}$ ) only the other minimum companion mass $M_{2} \sin i$ can be derived. The same is true for the semi-major axis $a$, which is projected with $i$ too.

In Table 1.1 the parameters to describe the configuration of a binary system are summarised 
Table 1.1.: Parameters to describe the configuration of a binary system.

\begin{tabular}{|c|c|c|}
\hline Parameter & Unit & Description \\
\hline$M_{1}, M_{\text {visible }}$ & $\mathrm{M}_{\odot}$ & Mass of the luminous (brighter) component. \\
\hline$M_{2}, M_{\text {invisible }}$ & $\mathrm{M}_{\odot}$ & Mass of the fainter component. \\
\hline$P$ & $\mathrm{~d}$ & Period of the orbit (same for both components). \\
\hline$e$ & - & Eccentricity of the orbit. \\
\hline$a$ & $\mathrm{AU}$ & Semi-major axis of the orbit. \\
\hline$i$ & $\circ$ & $\begin{array}{l}\text { Inclination of the orbit } \\
\left(0^{\circ} \text { is a face-on and } 90^{\circ} \text { an edge-on orbital plane). }\right.\end{array}$ \\
\hline$\phi$ & $\circ$ & Phase in time of the orbit. \\
\hline$\omega$ & $\circ$ & Argument of periapsis. \\
\hline$K, K_{1}$ & $\mathrm{~km} \mathrm{~s}^{-1}$ & $\begin{array}{l}\text { Semi-amplitude of the measurable radial velocities of the (usu- } \\
\text { ally) luminous component. }\end{array}$ \\
\hline$v_{\mathrm{r}}, v_{\mathrm{r}, 1}(t)$ & $\mathrm{km} \mathrm{s}^{-1}$ & $\begin{array}{l}\text { Time-dependent radial velocities of the (usually) luminous com- } \\
\text { ponent. }\end{array}$ \\
\hline
\end{tabular}

and are used throughout this work.

\subsection{Integral-field spectroscopy and MUSE}

A simple spectrograph is fed with one light source and disperses this light via a dispersion element (e.g. prism, grating, grism) into different wavelengths to obtain a spectrum on a screen or sensor. For multiple sources in the focal plane of a telescope there are different approaches to get the spectral information simultaneously for each source. A simple way is the so-called objective prism spectroscopy which uses a great prism or grating in the path of the light beam to disperse the light of every source. The offset of the sources is retained in the offset of the dispersed light on the sensor. Of course, this approach limits the spectral resolving power, the minimal offset between the sources and maximises the contaminating background. Another approach is to use multi-object spectrographs, where an aperture (fibre or optical element) selects a source and feeds it into a dedicated spectrograph or with high enough offsets in a single spectrograph to get independent spectra. These multi-object spectrographs are frequently used today and reach high spectral resolving powers. Their limit is of course a compromise between the size of the point spread function (PSF) of the source and the physical dimension of the aperture and their limited number. If PSFs overlap, multi-object spectrographs are usually not able to get individual spectra of blended sources. Therefore the next step further is to entirely divide the focal plane optically into small areas without gaps which are then send to spectrographs independently. These spectrographs are so-called integral-field spectrographs (IFSs) and use optical field splitter, slicer, lenslet arrays, or fibre arrays to divide the focal plane without compromise into a matrix. These spectrographs enable astronomers to get three dimensions of information - two spatial and one spectral - of a specific field of view (FoV). It does not really matter where the object in the FoV is and how extended the source is. Hence, blended, nearby sources can be 
untangled using the fully preserved spatial information. A side effect of these spectrographs is, that they are good in conserving the light flux, since most of the photons in the FoV should end on one of the sensors (apart from reflective or refractive imperfections and edge effects of the optical elements).

The first generation of IFSs, like SINFONI (Eisenhauer et al., 2003) or PMAS (Roth et al., 2005) had a relatively small FoV but demonstrated the performance of such spectrographs. For the second generation instrumentation of the Very Large Telescopes (VLTs) at the Paranal observatory in Chile of the European Southern Observatory (ESO) a consortium was founded to develop a next generation IFS called Multi Unit Spectroscopic Explorer (MUSE). It was commissioned in 2014 and combines the survey capabilities of an imager with the power of an IFS, while being very efficient ( $35 \%$ throughput at $7000 \AA$ ). In the wide field mode (WFM), MUSE covers a $1^{\prime} \times 1^{\prime}$ FoV with a spatial sampling of $0.2^{\prime \prime}$ and a spectral sampling of $1.25 \AA$ (resolving power of $1770<R<3590$ ) in the wavelength range from 4750 to $9350 \AA$ (Bacon et al., 2010). In parallel to the MUSE instrument a next generation adaptive optics module, Ground Atmospheric Layer Adaptive optiCs for Spectroscopic Imaging (GALACSI), was developed (Ströbele et al., 2012). It uses four sodium laser guide stars and a high speed and low noise wave front sensor to adjust the deformable secondary mirror of the Unit Telescope 4 at the VLT (VLT-UT4) to correct for the ground layer distortions by the atmosphere. Since October 2017 the MUSE instrument can use GALACSI to get sharper images (Leibundgut et al., 2017).

\subsection{MUSE survey of globular clusters}

\subsubsection{Pilot study}

During the commissioning of the MUSE instrument from July 26nd to August 3rd, 2014, a 5 x 5 mosaic of the central part of the globular cluster NGC 6397 was observed. In Husser et al. (2016) the capabilities of MUSE to do spectroscopy in this crowded field are presented. Using the precise positions of the stars from HST astrometry with the MUSE data cubes, a PSF-fitting technique was used to extract spectra of individual stars (Kamann et al., 2013). 18932 spectra of 10521 stars could be extracted, which excelled by far all spectroscopic surveys done in globular clusters to that time. It was shown that the spectra of in MUSE spatially blended sources could be successfully deblended from each other using the spatial and spectral information of the rich MUSE data. From the individual spectra the luminosity, effective temperature, radial velocity, and metallicity of each star could be derived with a full spectrum fit based on a grid of synthetic PHOENIX spectra (Husser et al., 2013). This enabled the creation of the first comprehensive spectroscopic HRD of a globular cluster. Thanks to the large sample of 7142 member stars tracing evolutionary states from the red giant branch to 4 magnitudes below the main sequence turnoff, it became statistically significant that the metallicity is changing along the branches with a minimum at the main sequence turnoff as expected. As a side effect two pecu- 
liar objects, a possible pulsar companion, and the subdwarf O star (sdO) ROB 162 were found in the data. This demonstrated that the survey capabilities of MUSE enabled spectroscopic discoveries, without knowing where to point the slit or fibre of a normal spectrograph.

The second publication by Kamann et al. (2016) refers to the internal dynamics of the globular cluster NGC 6397 with the aim to probe the presence of a central black hole. More effort was done to separate the star sample into cluster members and foreground stars by using the obtained spectroscopic information. The derived radial velocity measurements were calibrated, because they are crucial to do proper statistics with the whole radial velocity sample. It was shown that MUSE reaches a radial velocity accuracy of $1 \mathrm{~km} \mathrm{~s}^{-1}$. There was slight evidence of a rotational component in the cluster, that means the whole cluster system rotates, whereas individual star orbits still appear to be distributed randomly. Even though NGC 6397 was thought to be a core collapsed cluster, a mild central cusp in the velocity dispersion profile was found towards the cluster centre. A comparison with a JEANS model revealed that a black hole of $600 \mathrm{M}_{\odot}$ in the centre is in agreement with the data. However, other explanations for the velocity dispersion profile cusp, such as dark stellar components and thus an increasing mass-to-light ratio towards the cluster centre, are possible.

With these large samples of spectra, Wendt et al. (2017) showed that it is possible to probe the local interstellar medium (ISM) in line of sight between NGC 6397 and us. The absorption of, for example, Na I, K I and the so-called diffuse interstellar bands in the local bubble are responsible for some absorption features which appear in all spectra. Because of these multiple sight lines through the medium and the relatively large FoV of MUSE, maps of these absorption features could be made.

\subsubsection{A stellar census of globular clusters with MUSE}

After the successful commissioning of MUSE, a large guaranteed time observations (GTO) programme was started in 2014 to survey a large number of Galactic globular clusters. Table 1.2 lists the 26 GTO globular clusters (excluding NGC 6397) with their alternative name, position of the cluster centre, velocity dispersion, core radius, and the median number of observations (epochs) per pointing (as of June 2019). These globular clusters were chosen to have good visibility at Paranal Observatory and a high velocity dispersion which is resolvable with MUSE spectral resolution.

In 2018, after 4 years of MUSE observations on the GTO programme targets, Kamann et al. (2018b) published the first paper of a series presenting the results of the MUSE globular cluster survey. Using 500000 spectra of 200000 stars, with on average 9000 stars per cluster, the internal dynamics of 22 globular clusters were analysed. Preliminary results of my work were used to clean the dynamical sample from binaries, because they could falsify the results a bit. From that the dynamical distance could be estimated to 14 clusters, for which proper motion data was available. However, the main result of that study is, that a significant $(>3 \sigma)$ central rotation of 13 out of these 22 clusters could be detected. Furthermore, the study shows evidence for 
Table 1.2.: The MUSE Galactic globular cluster GTO targets.

\begin{tabular}{|c|c|c|c|c|c|c|}
\hline ID & Name & $\overline{\mathrm{RA}}$ & Dec. & $\begin{array}{l}\text { velocity dispersion } \\
\qquad \mathrm{km} \mathrm{s}^{-1}\end{array}$ & $\begin{array}{l}\text { core radius } \\
\text { pc }\end{array}$ & $\begin{array}{l}\text { epochs } \\
\text { median }\end{array}$ \\
\hline NGC 104 & 47 Tuc & $00^{\circ} 24^{\prime} 05^{\prime \prime} 67$ & $-72^{\circ} 04^{\prime} 52^{\prime \prime} 6$ & $11.0 \pm 0.3$ & 0.47 & 13 \\
\hline NGC 362 & & $01^{\circ} 03^{\prime} 14^{\prime \prime} 26$ & $-70^{\circ} 50^{\prime} 55^{\prime \prime} 6$ & $6.4 \pm 0.3$ & 0.45 & 2 \\
\hline NGC 1851 & & $05^{\circ} 14^{\prime} 06^{\prime \prime} 76$ & $-40^{\circ} 02^{\prime} 47^{\prime \prime} 6$ & $10.4 \pm 0.5$ & 0.32 & 7 \\
\hline NGC 1904 & M79 & $05^{\circ} 24$ 11."09 & $-24^{\circ} 31^{\prime} 29^{\prime \prime} 0$ & $5.3 \pm 0.4$ & 0.6 & 6 \\
\hline NGC 2808 & & $09^{\circ} 12^{\prime} 03^{\prime \prime} 10$ & $-64^{\circ} 51^{\prime} 48^{\prime \prime} 6$ & $13.4 \pm 1.2$ & 0.7 & 4 \\
\hline NGC 3201 & & $10^{\circ} 17^{\prime} 36^{\prime \prime} 82$ & $-46^{\circ} 24^{\prime} 44^{\prime \prime} 9$ & $5.0 \pm 0.2$ & 1.85 & 14 \\
\hline NGC 5139 & $\omega$ Cen & $13^{\circ} 26^{\prime} 47^{\prime \prime} \cdot 24$ & $-47^{\circ} 28^{\prime} 46^{\prime \prime} 5$ & $16.8 \pm 0.3$ & 3.58 & 12 \\
\hline NGC 5286 & & $13^{\circ} 46^{\prime} 26^{\prime \prime} 81$ & $-51^{\circ} 22^{\prime} 27^{\prime \prime} \cdot 3$ & $8.1 \pm 1.0$ & 0.94 & 4 \\
\hline NGC 5904 & M5 & $15^{\circ} 18^{\prime} 33^{\prime \prime} 22$ & $+02^{\circ} 04^{\prime} 51^{\prime \prime} .7$ & $5.5 \pm 0.4$ & 0.96 & 2 \\
\hline NGC 6093 & M80 & $16^{\circ} 17^{\prime} 02^{\prime \prime} 41$ & $-22^{\circ} 58^{\prime} 33^{\prime \prime} \cdot 9$ & $12.4 \pm 0.6$ & 0.44 & 2 \\
\hline NGC 6121 & M4 & $16^{\circ} 23^{\prime} 35^{\prime \prime} 22$ & $-26^{\circ} 31^{\prime} 32^{\prime \prime} 7$ & $4.0 \pm 0.2$ & 0.74 & 1 \\
\hline NGC 6218 & M12 & $16^{\circ} 47^{\prime} 14^{\prime \prime} 18$ & $-01^{\circ} 56^{\prime} 54^{\prime \prime} .7$ & $4.5 \pm 0.4$ & 1.1 & 3 \\
\hline NGC 6254 & M10 & $16^{\circ} 57^{\prime} 09^{\prime \prime} 05$ & $-04^{\circ} 06^{\prime} 01^{\prime \prime} 1$ & $6.6 \pm 0.8$ & 0.99 & 1 \\
\hline NGC 6266 & M62 & $17^{\circ} 01^{\prime} 12^{\prime \prime} 80$ & $-30^{\circ} 06^{\prime} 49^{\prime \prime} 4$ & $14.3 \pm 0.4$ & 0.44 & 3 \\
\hline NGC 6293 & & $17^{\circ} 10^{\prime} 10^{\prime \prime} 20$ & $-26^{\circ} 34^{\prime} 55^{\prime \prime} 5$ & $7.7 \pm 1.3$ & 0.14 & 2 \\
\hline NGC 6388 & & $17^{\circ} 36^{\prime} 17^{\prime \prime} \cdot 23$ & $-44^{\circ} 44^{\prime} 07^{\prime \prime} 8$ & $18.9 \pm 0.8$ & 0.35 & 4 \\
\hline NGC 6441 & & $17^{\circ} 50^{\prime} 13^{\prime \prime} 06$ & $-37^{\circ} 03^{\prime} 05^{\prime \prime} 2$ & $18.0 \pm 0.2$ & 0.44 & 4 \\
\hline NGC 6522 & & $18^{\circ} 03^{\prime} 34^{\prime \prime} 02$ & $-30^{\circ} 02^{\prime} 02^{\prime \prime} 3$ & $6.7 \pm 0.7$ & 0.11 & 3 \\
\hline NGC 6541 & & $18^{\circ} 08^{\prime} 02^{\prime \prime} 36$ & $-43^{\circ} 42^{\prime} 53^{\prime \prime} 6$ & $8.2 \pm 2.1$ & 0.39 & 2 \\
\hline NGC 6624 & & $18^{\circ} 23^{\prime} 40^{\prime \prime} 51$ & $-30^{\circ} 21^{\prime} 39^{\prime \prime} 7$ & $5.4 \pm 0.5$ & 0.14 & 2 \\
\hline NGC 6656 & M22 & $18^{\circ} 36^{\prime} 23^{\prime \prime} 94$ & $-23^{\circ} 54^{\prime} 17^{\prime \prime} 1$ & $7.8 \pm 0.3$ & 1.24 & 2 \\
\hline NGC 6681 & M70 & $18^{\circ} 43^{\prime} 12^{\prime \prime} 76$ & $-32^{\circ} 17^{\prime} 31^{\prime \prime} 6$ & $5.2 \pm 0.5$ & 0.08 & 2 \\
\hline NGC 6752 & & $19^{\circ} 10^{\prime} 52^{\prime \prime} 11$ & $-59^{\circ} 59^{\prime} 04^{\prime \prime} 4$ & $4.9 \pm 0.4$ & 0.2 & 2 \\
\hline NGC 7078 & M15 & $21^{\circ} 29^{\prime} 58^{\prime \prime} 33$ & $+12^{\circ} 10^{\prime} 01^{\prime \prime} \cdot 2$ & $13.5 \pm 0.9$ & 0.42 & 3 \\
\hline NGC 7089 & M2 & $21^{\circ} 33^{\prime} 27^{\prime \prime} 02$ & $-00^{\circ} 49^{\prime} 23^{\prime \prime} 7$ & $8.2 \pm 0.6$ & 1.07 & 4 \\
\hline NGC 7099 & M30 & $21^{\circ} 40^{\prime} 22^{\prime \prime} 12$ & $-23^{\circ} 10^{\prime} 47^{\prime \prime} .5$ & $5.5 \pm 0.4$ & 0.14 & 4 \\
\hline
\end{tabular}


a correlation between the internal rotation strengths and the relaxation times of the clusters. This means, that the rotation plays an important role during the evolution of a cluster: globular clusters are born with a high angular momentum which is then dissipated through two-body interactions to the observed amplitude today. The paper also demonstrated that MUSE achieves a radial velocity accuracy of $1-2 \mathrm{~km} \mathrm{~s}^{-1}$ and that radial velocity measurements could be reliably derived from spectra with low signal to noise $(\mathrm{S} / \mathrm{N}>5)$.

In October 2017 the adaptive optics (AO) system of MUSE was commissioned. A paper on cluster kinematics and stellar rotation was published by Kamann et al. (2018a) on the intermediate-age star cluster NGC 419 in the Small Magellanic Cloud (SMC) using data from the commissioning runs. The paper shows impressively how the spatial resolution of MUSE from 0.74 " improved to 0.35 " (at $8000 \AA$ ), when activating the AO system. This increased the number of stars and spectra in the final sample to 3321, covering all bright stars in the CMD of the MUSE FoV down to 1 magnitude below the main sequence turnoff. The analysis of these stars shows evidence for a difference in stellar rotation of $V \sin i \approx 40 \mathrm{~km} \mathrm{~s}^{-1}$ between the red and blue side of the main sequence. Also an indication for cluster rotation was found for this young (about 1.5 Gyr) SMC cluster.

During the investigation of emission line objects in globular clusters, Göttgens et al. (2019b) found a nova remnant in the globular cluster NGC 6656 (M 22). This is only the third nova remnant found in Galactic globular clusters and has an estimated mass of $1-17 \times 10^{-5} \mathrm{M}_{\odot}$. Surprisingly, the location of the nova coincides with a reported observation by ancient Chinese astronomers in May 48 BCE. Thus, this nova might be the oldest recorded and confirmed extra-solar observation in human history. A catalogue of $156 \mathrm{H} \alpha$ emission line objects with CVs, pulsating variable stars, eclipsing binary stars, the optical counterpart of a known black hole, and likely sub-subgiants (SSGs) or red stragglers (RSs) has been published in Göttgens et al. (2019a). This study was carried out at the same time as my study (the second paper in Chapter 2) and many results from the analyses of the binary stars of all globular clusters in our sample have been incorporated into the emission line catalogue. Both emission line papers demonstrate the efficiency of MUSE in detecting nebulae and single emission lines in spectra.

In the field of multiple populations (see Sect. 1.1.6), Latour et al. (2019) published a paper about single element abundances in the combined spectra of the four cluster populations of NGC 2808. My contribution was the matching of the newer HST photometry with bluer filters (Nardiello et al., 2018, Piotto et al., 2015) with our ACS photometry and the implementation of the "chromosome map" method to separate the different cluster populations. The variations in the spectral lines of oxygen, sodium, magnesium and aluminium are detected. Despite the low spectral resolution, MUSE is capable of reproducing the results of studies with high spectral resolution in the context of multiple populations. Furthermore, Husser et al. (2020) used the Ca II triplet (CaT) on individual spectra to extend the CaT-metallicity relation below the horizontal branch (HB). With this relation it is possible to infer the metallicity of RGB stars just from the CaT with an average uncertainty of about $0.12 \mathrm{dex}$. The metallicity distributions of 25 globular 
clusters are investigated and the metallicity differences of the multiple populations studied in more detail. The CaT-metallicity relation is also calibrated based on absolute magnitude and luminosity which could be used literally outside the field of globular clusters for field stars with known absolute magnitude, like the stars Gaia observes.

\subsection{Aims of this work}

The MUSE survey of globular clusters holds a unique data treasure. As introduced in Sect. 1.1.3, binaries are of elementary importance for the understanding of globular clusters. The aim of this work is the first all-encompassing spectroscopic study of binary stars in globular clusters. This work includes the assurance of a meaningful determination of radial velocities from the observation till the final analyses. For example, to get a better phase coverage of short period binaries, the observation strategy to have two observations per night and pointing for some clusters was introduced. One aim of this work is to ensure that systematic effects, during the reduction of MUSE observations and the extraction of spectra from these reductions, which affect the radial velocities and their uncertainties, are avoided. The determination of the radial velocities and their uncertainties themselves must be robust and reliable. A way has to be found how to analyse the radial velocities of individual stars automatically and to detect the variability of a star. Other effects which could cause radial velocity variations should be considered in the detection process of a binary system.

MUSE enables for the first time a complete systematic spectroscopic study of binaries and their properties in globular clusters. As there is no comparable study, this is breaking new ground. The research plan at the beginning of the PhD project (29 June 2016) was to use the multi-epoch observations of globular clusters with MUSE to address three aspects:

1. To infer the observational binary fraction of globular clusters using the radial velocity technique on individual stars in the FoV of MUSE.

2. To study the blue straggler star binarity of some globular clusters in more detail and to get some clue on their formation scenario.

3. To study the orbital parameters (period, eccentricity, host and companion mass) of a representative sample of binaries in more detail.

During the PhD project all three points have been investigated. In addition to this, black holes in globular clusters have become an important part of this work. It was previously not known whether BHs in binary systems in globular clusters would be detectable within our FoVs, which is why the first publication in Chapter 2 concentrates on the first $\mathrm{BH}$ found by our blind spectroscopic survey. We demonstrated that 20 radial velocity measurements are sufficient to infer the orbital parameters of the binary system reliably and to prove the unseen companion to be a stellar-mass $\mathrm{BH}$ without any prior knowledge. The second publication in 
Chapter 2 addresses not only all three research aspects together on NGC 3201, but also shows how to use advanced globular cluster models to translate a biased observational binary fraction into the total binary fraction of a globular cluster. Furthermore, thanks to the combination of many different previous works with our data, not only blue straggler stars (BSSs) but also sub-subgiants (SSGs) have been studied in more detail. For a significant sample of 95 binary stars the orbital parameters could be derived and a representative period-eccentricity plot has been created for a globular cluster for the first time. Finally, the third publication in Chapter 2 gives insight into the binary fraction of the two photometric distinguishable populations of NGC 3201, which reveals a significant difference between population 1 and 2, pointing towards a different dynamical evolution of the two populations.

The publication list might give the impression, that only the globular cluster NGC 3201 has been studied, but this is not true. The radial velocity (see the second paper in Chapter 2) and differential photometry (see Sect. A.1) methods have been applied to all clusters with enough observations. The problem is, as has been explained in detail in the second paper in Chapter 2, that sophisticated models are necessary to translate observational binary fractions to real binary fractions. Nevertheless, Appendix A contains some intermediate results obtained for other globular clusters. That part also contains other useful methods which were developed during the $\mathrm{PhD}$ project.

Finally this work ends with conclusions and an outlook in Chapter 3. 



\section{Publications}

This chapter presents publications as originally published, reprinted with permission from the corresponding publishers. The copyright of the original publications is held by the respective copyright holders, see the following copyright notices. In order to fit the paper dimension, reprinted publications may be scaled in size and/or cropped.

Giesers et al. (2018) (C) 2018 MNRAS. Reprinted with permission of the Oxford University Press. The original publication is available at MNRAS (https://doi.org/10.1093/ mnrasl/slx203).

S. Kamann contributed to the introduction and G. Anglada Escudé did the likelihood periodogram analysis and plot.

Giesers et al. (2019) (C) 2019 ESO. With permission from Astronomy \& Astrophysics. The original publication is available at A\&A (https://doi.org/10.1051/0004-6361/ 201936203).

A. Askar did the numerical simulation and contributed to the description of this model in Section 4.1.

Kamann et al. (2020) (C) 2020 ESO. Reprinted with kind permission of Sebastian Kamann and Astronomy \& Astrophysics. The original publication is available at A\&A (https:// doi.org/10.1051/0004-6361/201936843).

This paper was written by S. Kamann. My main contributions are the statistical and individual analyses on the radial velocity sample of the RGB stars, the creation of Figure 1., and discussions in Section 3.3. 



\title{
A detached stellar-mass black hole candidate in the globular cluster NGC 3201
}

\author{
Benjamin Giesers, ${ }^{1 \star}$ Stefan Dreizler, ${ }^{1 \star}$ Tim-Oliver Husser, ${ }^{1}$ Sebastian Kamann, ${ }^{1,2}$ \\ Guillem Anglada Escudé, ${ }^{3}$ Jarle Brinchmann, ${ }^{4,5}$ C. Marcella Carollo, ${ }^{6}$ \\ Martin M. Roth, ${ }^{7}$ Peter M. Weilbacher ${ }^{7}$ and Lutz Wisotzki ${ }^{7}$ \\ ${ }^{1}$ Institut für Astrophysik, Georg-August-Universität Göttingen, Friedrich-Hund-Platz 1, D-37077 Göttingen, Germany \\ ${ }^{2}$ Astrophysics Research Institute, Liverpool John Moores University, 146 Brownlow Hill, Liverpool L3 5RF, UK \\ ${ }^{3}$ School of Physics and Astronomy, Queen Mary University of London, 327 Mile End Road, EI 4NS London, UK \\ ${ }^{4}$ Leiden Observatory, Leiden University, PO Box 9513, NL-2300 RA, Leiden, the Netherlands \\ ${ }^{5}$ Instituto de Astrofísica e Ciências do Espaço, Universidade do Porto, CAUP, Rua das Estrelas, PT4150-762 Porto, Portugal \\ ${ }^{6}$ Institute for Astronomy, Swiss Federal Institute of Technology (ETH Zurich), CH-8093 Zurich, Switzerland \\ ${ }^{7}$ Leibniz-Institut für Astrophysik Potsdam (AIP), An der Sternwarte 16, D-14482 Potsdam, Germany
}

\begin{abstract}
As part of our massive spectroscopic survey of 25 Galactic globular clusters with MUSE, we performed multiple epoch observations of NGC 3201 with the aim of constraining the binary fraction. In this cluster, we found one curious star at the main-sequence turn-off with radial velocity variations of the order of $100 \mathrm{~km} \mathrm{~s}^{-1}$, indicating the membership to a binary system with an unseen component since no other variations appear in the spectra. Using an adapted variant of the generalized Lomb-Scargle periodogram, we could calculate the orbital parameters and found the companion to be a detached stellar-mass black hole with a minimum mass of $4.36 \pm 0.41 \mathrm{M}_{\odot}$. The result is an important constraint for binary and black hole evolution models in globular clusters as well as in the context of gravitational wave sources.
\end{abstract}

Key words: black hole physics - techniques: imaging spectroscopy-techniques: radial velocities - binaries: spectroscopic-globular clusters: individual: NGC 3201.

\section{INTRODUCTION}

Owing to their old ages and high masses, Galactic globular clusters probably have produced a large number of stellar-mass black holes during their lifetimes. Nevertheless, there is an ongoing debate about the number of black holes that actually remain in the cluster. In the absence of continuous star formation, stellar-mass black holes soon become the most massive objects in the cluster, where they accumulate around the centres as a consequence of mass segregation. However, because of the high mass-ratio with respect to the surviving low-mass stars $(\gtrsim 4: 1)$, it is expected that the black holes form a dense nucleus that is decoupled from the dynamics of the remaining cluster (Spitzer 1969). Interactions within this nucleus are then expected to eject the majority of black holes, so that only few survive after 1 Gyr (Kulkarni, Hut \& McMillan 1993; Sigurdsson \& Hernquist 1993).

However, over the past years, radio observations have revealed several sources in extragalactic and Galactic globular clusters that

^E-mail: giesers@astro.physik.uni-goettingen.de (BG); dreizler@astro. physik.uni-goettingen.de (SD) are likely to be stellar-mass black holes according to their combined radio and X-ray properties (Maccarone et al. 2007; Strader et al. 2012; Chomiuk et al. 2013). Under the assumption that only a small fraction of the existing black holes are actively accreting matter from a companion (Kalogera, King \& Rasio 2004), these detections point to much richer black hole populations in globular clusters than previously thought. In fact, state-of-the art models for clusters do predict that the retention fractions of black holes might be significantly enhanced compared to the earlier studies mentioned above (e.g. Breen \& Heggie 2013; Morscher et al. 2013). The reason for this is that the Spitzer (1969) instability only develops partially and the black hole nucleus does not detach from the kinematics of the remaining cluster. As a consequence, the evaporation time-scale is prolonged.

The search for black holes in globular clusters has recently gained further importance through the first detection of gravitational waves, produced by the coalescence of two massive black holes (Abbott et al. 2016a). As shown by Abbott et al. (2016c) or Askar et al. (2017), dense star clusters represent a preferred environment for the merging of such black hole binaries. Hence, it would be crucial to overcome the current observational limits in order to increase our sample of known black holes. Compared to radio or X-ray studies, dynamical searches for stellar companions have the advantages of 


\section{L16 B. Giesers et al.}

also being sensitive to non-accreting black holes and of providing direct mass constraints. We are currently conducting a large survey of 25 Galactic globular clusters with MUSE (Multi Unit Spectroscopic Explorer, Bacon et al. 2010), which provides us with spectra of currently 600 to 27000 stars per cluster (see Kamann et al. 2017). Our survey includes a monitoring for radial velocity variations, which is very sensitive to the detection of stellar companions of massive objects (i.e. black holes, neutron stars and white dwarfs). Here, we report the detection of a $4.36 \pm 0.41 \mathrm{M}_{\odot}$ black hole in the globular cluster NGC 3201

\section{OBSERVATIONS AND DATA REDUCTION}

The observational challenge in globular clusters is the crowding resulting in severe blending of nearby stars especially in the cluster cores. For photometric measurements of dense globular clusters, instruments like the Hubble Space Telescope (HST) provide sufficient spatial resolution to obtain independent measurements of most of the stars (Sarajedini et al. 2007). For spectroscopic surveys, investigations of dense stellar fields were limited to the brightest stars or to regions with sufficiently reduced crowding. In globular clusters, the combination of field of view $\left(1 \times 1 \mathrm{arcmin}^{2}\right)$ and spatial sampling $\left(300 \times 300\right.$ spaxel $\left.^{2}\right)$ of MUSE allows us to extract spectra with a spectral resolution of $1800<R<3500$ of some thousand stars per exposure (for more details see Husser et al. 2016). We use the standard ESO MUSE pipeline to reduce the MUSE raw data (Weilbacher et al. 2012). The extraction is done with a PSF-fitting technique using the combined spatial and spectral information (Kamann et al. 2013, 2014).

This work is part of our investigation of the binarity of clusters using the radial velocity method. Binaries that are able to survive in the dense environment of a globular cluster are so tight (see Hut et al. 1992) that even HST is unable to resolve the single components such that they will appear as a single point-like source. Radial velocity surveys, however, will rather easily detect those compact binary systems. Except for the case when both stars have the same brightness, the extracted MUSE spectrum will be dominated by one of the stars, i.e. we mostly detect single line binary systems. With the knowledge of the stellar mass and the orbital parameters from our analyses, we can nevertheless infer the minimum masses of the unseen companions.

Up to this publication, we have observed three globular cluster NGC 104 (47 Tuc), NGC 3201, and NGC 5139 ( $\omega$ Cen) with sufficiently many observations to analyse the radial velocity signal $v_{r}$ of individual stars. We identified three stars with radial velocity variations exceeding $100 \mathrm{~km} \mathrm{~s}^{-1}$. Only one of them (hereafter called target star) in NGC 3201 appears in two overlapping pointings, resulting in 20 extracted spectra with good signal-to-noise ratios (from 24 to 56,43 on average). The other two stars need more observations to analyse the orbital parameters. Table 1 lists the target star's derived radial velocities and seeing values for each observation from five observation runs.

\section{PHOTOMETRIC AND SPECTROSCOPIC ANALYSIS}

The investigation of the target star properties is performed in two steps. Photometry provides the mass of the target star and allows us to exclude alternative explanations for the large radial velocity signal. Below, we describe the spectral analysis from which we obtain the cluster membership.
Table 1. Barycentric corrected radial velocity $v_{r}$, (Vega) magnitude $I_{F 814 W}$, and seeing (See.) measurements for the target star. ( $\triangle \mathrm{JD}$ : Julian observation date JD - 2456978 d, ESO ID: ESO Programme ID Code.)

\begin{tabular}{lcccc}
\hline$\Delta \mathrm{JD}(\mathrm{d})$ & $v_{r}\left(\mathrm{~km} \mathrm{~s}^{-1}\right)$ & $I_{F 814 W}(\mathrm{mag})$ & See. $(\operatorname{arcsec})$ & ESO prog. ID \\
\hline 0.83733 & $570.8 \pm 2.2$ & $16.88 \pm 0.08$ & 0.82 & 094.D-0142 \\
11.85794 & $512.9 \pm 2.7$ & $16.89 \pm 0.06$ & 0.90 & 094.D-0142 \\
11.86438 & $511.9 \pm 2.8$ & $16.91 \pm 0.08$ & 0.84 & 094.D-0142 \\
30.81091 & $475.0 \pm 2.2$ & $16.83 \pm 0.06$ & 0.60 & 094.D-0142 \\
30.83250 & $482.1 \pm 2.4$ & $16.88 \pm 0.07$ & 0.66 & 094.D-0142 \\
31.78096 & $477.1 \pm 2.0$ & $16.86 \pm 0.06$ & 0.72 & 094.D-0142 \\
31.80265 & $480.8 \pm 2.1$ & $16.87 \pm 0.07$ & 0.88 & 094.D-0142 \\
149.49256 & $536.8 \pm 2.0$ & $16.90 \pm 0.06$ & 0.60 & 095.D-0629 \\
151.47767 & $550.8 \pm 3.6$ & $16.82 \pm 0.07$ & 1.12 & 095.D-0629 \\
153.47698 & $559.3 \pm 2.5$ & $16.86 \pm 0.08$ & 1.00 & 095.D-0629 \\
156.47781 & $585.4 \pm 2.2$ & $16.86 \pm 0.08$ & 1.04 & 095.D-0629 \\
160.47808 & $609.5 \pm 2.0$ & $16.87 \pm 0.08$ & 0.70 & 095.D-0629 \\
441.74475 & $476.2 \pm 2.0$ & $16.90 \pm 0.06$ & 0.66 & 096.D-0175 \\
441.76738 & $472.0 \pm 1.8$ & $16.88 \pm 0.07$ & 0.64 & 096.D-0175 \\
443.74398 & $474.8 \pm 2.1$ & $16.89 \pm 0.06$ & 0.66 & 096.D-0175 \\
443.76792 & $472.5 \pm 2.1$ & $16.85 \pm 0.08$ & 0.54 & 096.D-0175 \\
538.47410 & $471.1 \pm 1.9$ & $16.86 \pm 0.06$ & 0.80 & 097.D-0295 \\
542.47958 & $471.9 \pm 2.0$ & $16.88 \pm 0.06$ & 0.78 & 097.D-0295 \\
808.87270 & $501.4 \pm 2.7$ & $16.80 \pm 0.06$ & 0.60 & 098.D-0148 \\
809.87675 & $512.7 \pm 2.5$ & $16.87 \pm 0.08$ & 0.96 & 098.D-0148 \\
\hline
\end{tabular}

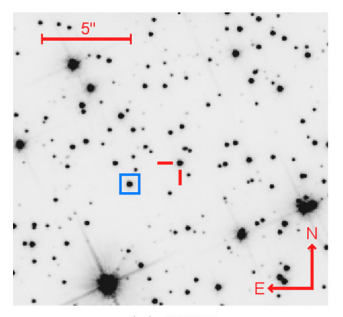

(a) HST

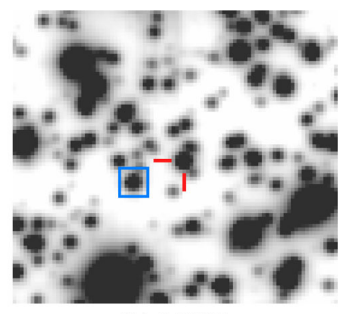

(b) MUSE
Figure 1. Charts with the target star marked with red cross-hairs and the reference star marked within a blue square. (a) The image is taken from the HST ACS globular cluster survey from Sarajedini et al. (2007) and Anderson et al. (2008). (b) Same field of view seen by MUSE with a seeing of 0.6 arcsec. The displayed image is a cut $(90 \times 81$ pixels $)$ from an integrated white light image of the MUSE data cube.

\subsection{Photometric analysis}

Fig. 1 shows the target star (red cross-hairs) with a magnitude of $I_{F 814 \mathrm{~W}}=16.87 \mathrm{mag}$ (Vega) and its nearby stars from HST ACS data (Anderson et al. 2008). To monitor the reliability of our analyses, we have picked the star in the blue square with a magnitude of $I_{F 814 W}=17.11$ as a reference star $\left(\mathrm{RA}=10^{\mathrm{h}} 17^{\mathrm{m}} 37^{\mathrm{s}} .36\right.$, Dec. $\left.=-46^{\circ} 24^{\prime} 56^{\prime \prime} 48\right)$. The three nearest stars have magnitudes fainter than 19 mag. For a consistency check and for investigating photometric variability, we convolve each flux calibrated spectrum with the corresponding ACS filter function and compare it to the ACS photometry. For all extracted spectra, we reach a magnitude accuracy of at least 95 per cent compared to the HST ACS magnitude (see Table 1). This indicates that the target star is extracted without contamination.

In order to derive the mass and surface gravity $\log g$ for the spectrum fit (see Table 2) of the target star, we compare its HST ACS colour and magnitude with a PARSEC isochrone (Bressan et al. 2012). For the globular cluster NGC 3201, we found the 
Table 2. Target star properties. The position and magnitude were taken from the HST ACS globular cluster survey catalogue (Anderson et al. 2008), mass and surface gravity are derived from isochrone fitting, effective temperature and metallicity are derived from spectral fitting. For more details see Section 3.

\begin{tabular}{lccc}
\hline RA & $10^{\mathrm{h}} 17^{\mathrm{m}} 37^{\mathrm{s}} .090$ & Dec. & $-46^{\circ} 24^{\prime} 55^{\prime \prime} \cdot 31$ \\
\hline$I_{F 814 W}$ & $(16.87 \pm 0.02) \mathrm{mag}($ Vega $)$ & & \\
$M$ & $(0.81 \pm 0.05) \mathrm{M} \odot$ & $\log g$ & $(3.99 \pm 0.05) \mathrm{dex}$ \\
$T_{\text {eff }}$ & $(6126 \pm 20) \mathrm{K}$ & {$[\mathrm{M} / \mathrm{H}]$} & $(-1.50 \pm 0.02) \mathrm{dex}$ \\
\hline
\end{tabular}

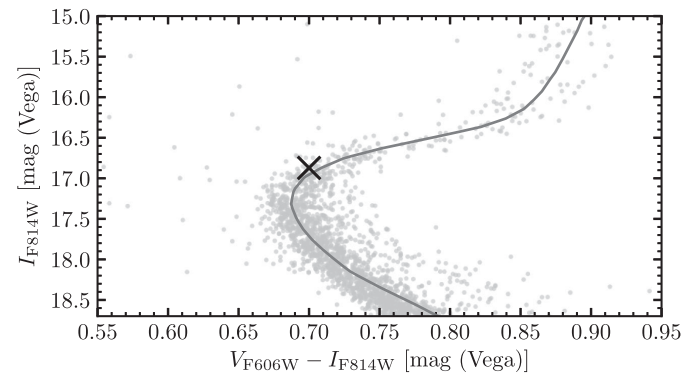

Figure 2. CMD of the globular cluster NGC 3201 created from the HST ACS photometry of Anderson et al. (2008). The target star position is highlighted by a black $\mathrm{X}$. The line represents our best-fitting PARSEC isochrone (Bressan et al. 2012, for more details see Section 3.1).

best matching isochrone compared to the whole HST ACS Colourmagnitude diagram $(\mathrm{CMD})$ with the isochrone parameters $[\mathrm{M} / \mathrm{H}]=$ -1.39 dex (slightly above the comparable literature value $[\mathrm{Fe} / \mathrm{H}]$ $=-1.59 \mathrm{dex}$, Harris 1996), age $=11 \mathrm{Gyr}$, extinction $E_{B-V}=0.26$, and distance $=4.8 \mathrm{kpc}$ (see Fig. 2). The target star is at the mainsequence turn-off with a mass of $0.81 \pm 0.05 \mathrm{M}_{\odot}$ as estimated from the isochrone.

Although the position of the target star in the CMD is not in the classical instability strip, we want to exclude radial velocity variations caused by photometric variability (e.g. due to pulsations) Therefore, we use the differential photometry method on our MUSE data. We first run an iterative algorithm to find a large sample of reference stars (which are present in all observations). After these stars have been identified, normally with an intrinsic standard deviation of $0.005 \mathrm{mag}$ in the filter $I_{\text {Bessel }}$ reconstructed from the extracted spectrum, we compare each star of each observation with our reference stars selecting 20 with comparable colour. The photometric analysis for the target star shows no significant variation (see Table 1). We would expect significant changes in the brightness of the target star to explain such a high radial velocity amplitude via pulsations. We therefore conclude that the radial velocity variations are not intrinsic to the target star. The absence of large photometric variations also suggests the absence of an interacting binary. We like to note that no radio or X-ray source is known at the target star's position. For instance, Strader et al. (2013) performed a deep systematic radio continuum survey for black holes also in NGC 3201 , but did not publish any discovery. Some X-ray sources were found by Webb, Wheatley \& Barret (2006), but none at the target star's coordinates.
Black hole candidate in NGC 3201

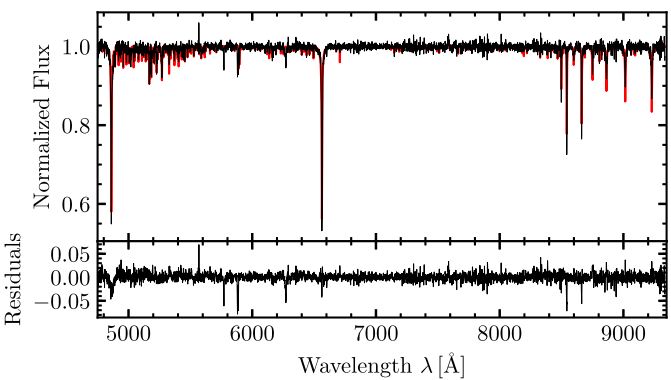

Figure 3. The combination of all radial velocity corrected spectra of the target star (in black). The best-fitting PHOENIX spectrum is indicated in the background in red (thicker curve for better visibility). The bottom panel shows the residuals after subtracting the best fit to the data.

\subsection{Spectroscopic analysis}

After the extraction of point sources, the individual spectra of the 20 visits are fitted against our Göttingen Spectral Library (Husser et al. 2013) of synthetic PHOENIX spectra to determine the stellar effective temperature and metallicity as well as radial velocities and the telluric absorption spectrum. The simultaneous fit of stellar and telluric spectra is performed with a least-squares minimization comparing the complete observed spectrum against our synthetic spectra. The telluric absorptions are used to correct for small remaining wavelength calibration uncertainties, allowing us to reach a radial velocity precision down to $1 \mathrm{~km} \mathrm{~s}^{-1}$. For more details about the stellar parameter fitting methods, we refer to Husser et al. (2013, 2016). We find huge changes in radial velocity between the spectra of up to $138 \mathrm{~km} \mathrm{~s}^{-1}$. We got the same variation if we do just cross-correlations between the spectra and the initial template, of course with higher uncertainties. Besides the radial velocity signal, the spectral fitting of the individual spectra did not reveal any other significant variations. The radial velocities are given in Table 1, whereas the mean stellar parameters are given in Table 2 .

Using the derived radial velocities, the individual spectra are placed into rest frame and are then combined using the drizzling method from Fruchter \& Hook (2002) and finally normalized to the continuum. The combined spectrum in Fig. 3 is compared to one of our PHOENIX spectra for a star at the main-sequence turn-off in the globular cluster NGC 3201. The spectral properties match the position of the star in the CMD displayed in Fig. 2. We note that there are no emission lines that could indicate, for example, a cataclysmic variable or a compact binary with an illuminated low-mass star and a hot companion like a white dwarf or neutron star.

The mean radial velocity $506 \pm 1 \mathrm{~km} \mathrm{~s}^{-1}$ and mean metallicity $[\mathrm{M} / \mathrm{H}]=-1.50 \pm 0.02$ dex of the target star is in good agreement with the cluster parameters in the Harris (1996) catalogue. Further, the fitted radial velocity of the binary barycentre (see Table 3 ) matches precisely the radial velocity of the cluster $494.0 \pm 0.2 \mathrm{~km} \mathrm{~s}^{-1}$. This makes the target star a bonafide cluster member.

\section{RESULTS}

The analysis of the radial velocity variation is done using the generalized Lomb-Scargle (GLS) periodogram (Zechmeister \& Kürster 2009), the likelihood function approach of Baluev (2008), and a final fit of a Keplerian orbit. Fig. 4 shows the likelihood periodogram of 


\section{L18 B. Giesers et al.}

Table 3. Binary system properties. The Keplerian parameters were calculated with an MCMC consisting of $10^{7}$ iterations. The derived mass was calculated using $2.0 \times 10^{4} \mathrm{MCMC}$ samples and the same number of target star mass samples.

\begin{tabular}{ll}
\hline Period $P$ & $166.88_{-0.63}^{+0.71} \mathrm{~d}$ \\
\hline Doppler semi-amplitude $K$ & $(69.4 \pm 2.5) \mathrm{km} \mathrm{s}^{-1}$ \\
Eccentricity $e$ & $0.595 \pm 0.022$ \\
Argument of periastron $\omega$ & $(2.6 \pm 3.2)^{\circ}$ \\
Periastron passage $T_{0}$ & $(57140.2 \pm 0.5) \mathrm{d}$ \\
Barycentric radial velocity $\gamma_{0}$ & $(494.5 \pm 2.4) \mathrm{km} \mathrm{s}^{-1}$ \\
Linear trend $\dot{\gamma}$ & $(-0.27 \pm 2.70) \mathrm{km} \mathrm{s}^{-1}$ \\
Jitter $s$ & $0.68_{-0.25}^{+0.40} \mathrm{~km} \mathrm{~s}^{-1}$ \\
Minimum companion mass $M \sin (i)$ & $(4.36 \pm 0.41) \mathrm{M}_{\odot}$ \\
Minimum semi-major axis $a(M)$ & $(1.03 \pm 0.03) \mathrm{au}$ \\
\end{tabular}

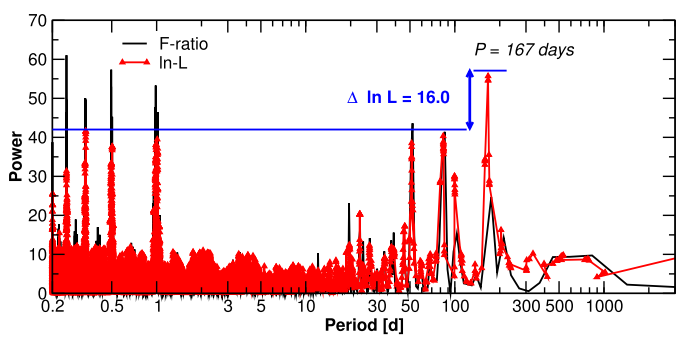

Figure 4. Likelihood periodogram of the radial velocities of the target star The black curve is a version of the GLS periodogram for circular orbit using the F-ratio statistic to represent the significance of each solution. The red triangles show the improvement on the log-likelihood statistic using a full Keplerian fit at the period search level instead.

the target star for the period range $0.2-1000 \mathrm{~d}$. The black curve (F-ratio) represents the GLS periodogram for circular orbits. It shows highly significant periods at $1 \mathrm{~d}$, fractions of $1 \mathrm{~d}, 51 \mathrm{~d}$, and $83 \mathrm{~d}$. The $1 \mathrm{~d}$ period and fractions of it are aliases of our nightly observation basis. With Keplerian fits for the same period range, the resulting picture is different. The red triangles (ln-L curve) show these Keplerian fits as a log-likelihood statistic. The $167 \mathrm{~d}$ period has a very low false-alarm probability of $2.2 \times 10^{-8}$, so the signal is extremely significant. Compared to all other peaks, the ln-likelihood of the preferred solution is higher by $\Delta \ln L=16$. Within the framework of this type of periodogram, this implies a $\sim 8.9 \times 10^{6}$ higher probability. Moreover, the $51 \mathrm{~d}$ and $83 \mathrm{~d}$ peaks are most likely only harmonics of this period $(83 \mathrm{~d} \approx 167 \mathrm{~d} / 2)$ and the window function, which has a high power at $135 \mathrm{~d}(1 / 51-1 / 83 \approx 1 / 135)$. We also performed a detection probability test comparable to Fiorentino et al. (2010) to verify that our sampling is sensitive to all periods in the probed range.

Fig. 5 shows the radial velocity measurements phase folded with the $167 \mathrm{~d}$ period and the best-fitting Keplerian orbit solution. The reduced $\chi^{2}$ of the Keplerian orbit fit is 1.2 (for comparison the reduced $\chi^{2}$ of the best circular orbit at the $83 \mathrm{~d}$ period is 45 ). The resulting orbital parameters fitted with the MCMC approach of Haario et al. (2006) are shown in Table 3. The reference star does not show any significant radial velocity variation (reduced $\chi^{2}=0.42$ ).

\section{DISCUSSION AND CONCLUSIONS}

The data show strong evidence that the target star is in a binary system with a non-luminous object having a minimum mass of

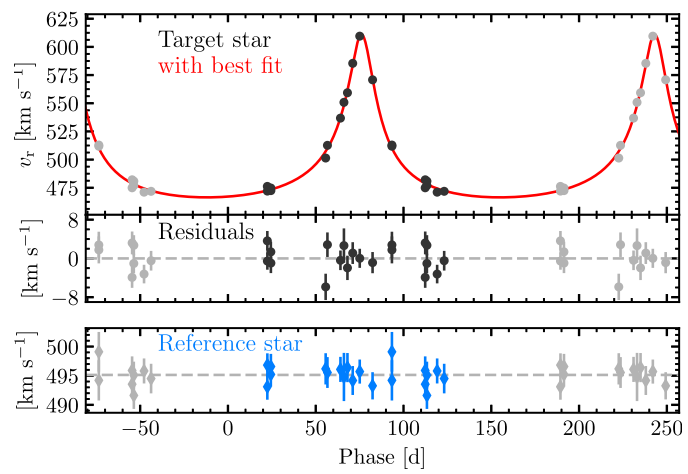

Figure 5. The top panel shows the radial velocity measurements $v_{r}$ of the target star, phase folded for the $167 \mathrm{~d}$ period. Error bars are smaller than the data points. The red curve shows the best-fitting Keplerian orbit (see Table 3). The middle panel contains the residuals after subtracting this best-fitting model to the data. The bottom panel shows the radial velocity measurements of the reference star. Grey dots are phase shifted duplicates of the black ones, and are included to improve the visualization.

$4.36 \pm 0.41 \mathrm{M}_{\odot}$. This object should be degenerate, since it is invisible and the minimum mass is significantly higher than the Chandrasekhar limit $\left(\sim 1.4 \mathrm{M}_{\odot}\right)$. The small distance of the system from the centre of the globular cluster $(10.8$ arcsec) is expected from mass segregation. Most likely, the degenerate object has exceeded the Tolman-Oppenheimer-Volkoff limit that predicts all objects to collapse into black holes above $\sim 3 \mathrm{M}_{\odot}$ (Bombaci 1996). We note that the mass estimate of the dark companion depends only weakly on the mass of the target star within reasonable error estimates (e.g. for the unrealistic case of a target star with $0.2 \mathrm{M}_{\odot}$, the minimum companion mass will still be above $3 \mathrm{M}_{\odot}$ ).

Alternatively, the discovery could eventually be explained through a triple star system that consists of a compact double neutron star binary with a main-sequence turn-off star around it. In the literature, neutron star binaries show a narrow mass distribution of $1.35 \pm 0.04 \mathrm{M}_{\odot}$ per star (Thorsett \& Chakrabarty 1999; Lattimer 2012). Recent discoveries show that a single neutron star could reach $2.0 \mathrm{M}_{\odot}$ (Özel \& Freire 2016). Thus, a double neutron star system with both components having more than $2.0 \mathrm{M}_{\odot}$ could explain the observations. Since such a system was not observed to date and the actual mass of the discovered object is probably higher, a black hole scenario is more likely. In this case, our results represent the first direct mass estimate of a (detached) black hole in a globular cluster.

The recent discovery of coalescing black hole binaries (Abbott et al. 2016b) suggests that there is a large population of stellar-mass binary black holes in the Universe. Our results confirm that the components of such binaries can be found in globular clusters.

The black hole is assumed to be detached because the closest possible approach in our best-fitting model is 0.4 au and the Roche limit for the target star with a reasonable radius of $1 \mathrm{R}_{\odot}$ is of the order of $3 \mathrm{R} \odot$. We have no evidence that the black hole accretes mass emits X-rays or radio jets.

Compared to other globular clusters, the most unusual structural parameter of NGC 3201 is the large cluster core radius (1.3 arcmin, see Harris 1996). As the presence of black holes can lead to an expansion of the core radius through interactions between black holes and stars (Strader et al. 2012), the discovery of the presented 
black hole could be an indication that NGC 3201 possesses an extensive black hole system in its core. More observations with MUSE could reveal more black hole companions using the radial velocity method.

To get the true system mass function, it is necessary to measure the inclination of the system. Since the Sun's distance to the globular cluster NGC 3201 is $4.9 \mathrm{kpc}$ (Harris 1996), the orbital movement of the target star is of the order of 0.2 mas. This should be observable with interferometers. Unfortunately, for the ESO interferometer VLTI GRAVITY, a reference star with K 11 mag within 2 arcsec is missing (Gillessen et al. 2010). Maybe it could be observable with diffraction limited large telescopes like HST, the VLT with NACO at UT1, or the VLT with the new adaptive optics facility (GRAAL) at UT4. Certainly, this would be a nice astrometry task for the JWST and for the upcoming first-light instrument MICADO at the ELT (Massari et al. 2016).

\section{ACKNOWLEDGEMENTS}

BG, SD, SK, and PMW acknowledge support from the German Ministry for Education and Science (BMBF Verbundforschung) through grants 05A14MGA, 05A17MGA, 05A14BAC, and 05A17BAA. GAE acknowledges a Gauss-Professorship granted by the Akademie für Wissenschaften zu Göttingen. JB acknowledges support by Fundação para a Ciência e a Tecnologia (FCT) through national funds (UID/FIS/04434/2013) and Investigador FCT contract IF/01654/2014/CP1215/CT0003, and by Fondo Europeo de Desarrollo Regional (FEDER) through COMPETE2020 (POCI-01-0145-FEDER-007672). This research is supported by the German Research Foundation (DFG) with grants DR 281/35-1 and KA 4537/2-1. Based on observations made with ESO Telescopes at the La Silla Paranal Observatory (see programme IDs in Table 1). Based on observations made with the NASA/ESA Hubble Space Telescope, obtained from the data archive at the Space Telescope Science Institute. STScI is operated by the Association of Universities for Research in Astronomy, Inc. under NASA contract NAS 5-26555. Supporting data for this article is available at: http://musegc.uni-goettingen.de.

\section{REFERENCES}

Abbott B. P. et al., 2016a, Phys. Rev. Lett., 116, 061102 Abbott B. P. et al., 2016b, Phys. Rev. Lett., 116, 241103 Abbott B. P. et al., 2016c, ApJ, 818, L22

Anderson J. et al., 2008, AJ, 135, 2055

Askar A., Szkudlarek M., Gondek-Rosińska D., Giersz M., Bulik T., 2017, MNRAS, 464, L36
Black hole candidate in NGC 3201

Bacon R. et al., 2010, Proc. SPIE Conf. Ser. Vol. 7735, The MUSE SecondGeneration VLT Instrument. SPIE, Bellingham, p. 8

Baluev R. V., 2008, MNRAS, 385, 1279

Bombaci I., 1996, A\&A, 305, 871

Breen P. G., Heggie D. C., 2013, MNRAS, 432, 2779

Bressan A., Marigo P., Girardi L., Salasnich B., Dal Cero C., Rubele S. Nanni A., 2012, MNRAS, 427, 127

Chomiuk L., Strader J., Maccarone T. J., Miller-Jones J. C. A., Heinke C. Noyola E., Seth A. C., Ransom S., 2013, ApJ, 777, 69

Fiorentino G. et al., 2010, ApJ, 708, 817

Fruchter A. S., Hook R. N., 2002, PASP, 114, 144

Gillessen S. et al., 2010, Proc. SPIE Conf. Ser. Vol. 7734, Optical and Infrared Interferometry II. SPIE, Bellingham, p. 77340Y

Haario H., Laine M., Mira A., Saksman E., 2006, Statistics and Computing, 16,339

Harris W. E., 1996, AJ, 112, 1487

Husser T.-O., Wende-von Berg S., Dreizler S., Homeier D., Reiners A. Barman T., Hauschildt P. H., 2013, A\&A, 553, A6

Husser T.-O. et al., 2016, A\&A, 588, A148

Hut P. et al., 1992, PASP, 104, 981

Kalogera V., King A. R., Rasio F. A., 2004, ApJ, 601, L171

Kamann S., Wisotzki L., Roth M. M., 2013, A\&A, 549, A71

Kamann S., Wisotzki L., Roth M. M., Gerssen J., Husser T.-O., Sandin C., Weilbacher P., 2014, A\&A, 566, A58

Kamann S. et al., 2017, MNRAS, 473, 559

Kulkarni S. R., Hut P., McMillan S., 1993, Nature, 364, 421

Lattimer J. M., 2012, Annual Review of Nuclear and Particle Science, 62 485

Maccarone T. J., Kundu A., Zepf S. E., Rhode K. L., 2007, Nature, 445, 183 Massari D. et al., 2016, Proc. SPIE Conf. Ser. Vol. 9909, Adaptive Optics Systems V. SPIE, Bellingham, p. 99091G

Morscher M., Umbreit S., Farr W. M., Rasio F. A., 2013, ApJ, 763, L15

Özel F., Freire P., 2016, ARA\&A, 54, 401

Sarajedini A. et al., 2007, AJ, 133, 1658

Sigurdsson S., Hernquist L., 1993, Nature, 364, 423

Spitzer L., Jr, 1969, ApJ, 158, L139

Strader J., Chomiuk L., Maccarone T. J., Miller-Jones J. C. A., Seth A. C., 2012, Nature, 490, 71

Strader J., Miller-Jones J., Maccarone T., Chomiuk L., 2013, The ATCA Survey for Black Holes in Southern Globular Clusters, ATNF Proposal

Thorsett S. E., Chakrabarty D., 1999, ApJ, 512, 288

Webb N. A., Wheatley P. J., Barret D., 2006, A\&A, 445, 155

Weilbacher P. M., Streicher O., Urrutia T., Jarno A., Pécontal-Rousset A., Bacon R., Böhm P., 2012, Proc. SPIE Conf. Ser. Vol. 8451, Software and Cyberinfrastructure for Astronomy II. SPIE, Bellingham, p. 84510B Zechmeister M., Kürster M., 2009, A\&A, 496, 577

This paper has been typeset from a $\mathrm{T}_{\mathrm{E}} \mathrm{X} / \mathrm{L} \mathrm{T} \mathrm{E} \mathrm{X}$ file prepared by the author. 

A\&A 632, A3 (2019)

https://doi.org/10.1051/0004-6361/201936203

(c) ESO 2019

\title{
Astronomy Astrophysics
}

\section{A stellar census in globular clusters with MUSE: Binaries in NGC 3201^}

\author{
Benjamin Giesers ${ }^{1}$, Sebastian Kamann ${ }^{2}$, Stefan Dreizler ${ }^{1}$, Tim-Oliver Husser ${ }^{1}$, Abbas Askar ${ }^{3}$, Fabian Göttgens ${ }^{1}$, \\ Jarle Brinchmann ${ }^{4,5}$, Marilyn Latour ${ }^{1}$, Peter M. Weilbacher ${ }^{6}$, Martin Wendt ${ }^{7}$, and Martin M. Roth ${ }^{6}$ \\ 1 Institut für Astrophysik, Georg-August-Universität Göttingen, Friedrich-Hund-Platz 1, 37077 Göttingen, Germany \\ e-mail: giesers@astro.physik.uni-goettingen.de \\ 2 Astrophysics Research Institute, Liverpool John Moores University, 146 Brownlow Hill, Liverpool L3 5RF, UK \\ ${ }^{3}$ Lund Observatory, Department of Astronomy and Theoretical Physics, Lund University, Box 43, 22100 Lund, Sweden \\ 4 Instituto de Astrofísica e Ciências do Espaço, Universidade do Porto, CAUP, Rua das Estrelas, 4150-762 Porto, Portugal \\ 5 Leiden Observatory, Leiden University, PO Box 9513, 2300 RA Leiden, The Netherlands \\ ${ }^{6}$ Leibniz-Institut für Astrophysik Potsdam (AIP), An der Sternwarte 16, 14482 Potsdam, Germany \\ ${ }^{7}$ Institut für Physik und Astronomie, Universität Potsdam, Karl-Liebknecht-Str. 24/25, 14476 Golm, Germany
}

Received 28 June 2019 / Accepted 17 September 2019

\section{ABSTRACT}

We utilise multi-epoch MUSE spectroscopy to study binary stars in the core of the Galactic globular cluster NGC 3201. Our sample consists of 3553 stars with 54883 spectra in total comprising 3200 main-sequence stars up to 4 magnitudes below the turn-off. Each star in our sample has between 3 and 63 (with a median of 14) reliable radial velocity measurements within five years of observations. We introduce a statistical method to determine the probability of a star showing radial velocity variations based on the whole inhomogeneous radial velocity sample. Using HST photometry and an advanced dynamical MOCCA simulation of this specific cluster we overcome observational biases that previous spectroscopic studies had to deal with. This allows us to infer a binary frequency in the MUSE field of view and enables us to deduce the underlying true binary frequency of $(6.75 \pm 0.72) \%$ in NGC 3201 . The comparison of the MUSE observations with the MOCCA simulation suggests a large portion of primordial binaries. We can also confirm a radial increase in the binary fraction towards the cluster centre due to mass segregation. We discovered that in the core of NGC 3201 at least $(57.5 \pm 7.9) \%$ of blue straggler stars are in a binary system. For the first time in a study of globular clusters, we were able to fit Keplerian orbits to a significant sample of 95 binaries. We present the binary system properties of eleven blue straggler stars and the connection to SX Phoenicis-type stars. We show evidence that two blue straggler formation scenarios, the mass transfer in binary (or triple) star systems and the coalescence due to binary-binary interactions, are present in our data. We also describe the binary and spectroscopic properties of four sub-subgiant (or red straggler) stars. Furthermore, we discovered two new black hole candidates with minimum masses $(M \sin i)$ of $(7.68 \pm 0.50) M_{\odot},(4.4 \pm 2.8) M_{\odot}$, and refine the minimum mass estimate on the already published black hole to $(4.53 \pm 0.21) M_{\odot}$. These black holes are consistent with an extensive black hole subsystem hosted by NGC 3201 .

Key words. binaries: general - blue stragglers - stars: black holes - techniques: radial velocities techniques: imaging spectroscopy - globular clusters: individual: NGC 3201

\section{Introduction}

Multi-star systems are not only responsible for an abundance of extraordinary objects like cataclysmic variables, millisecond pulsars, and low-mass X-ray binaries, but can also be regarded as a source of energy: Embedded in the environment of other stars, like in star clusters, the gravitational energy stored in a multi-star system can be transferred to other stars. In globular clusters with stellar encounters on short timescales this energy deposit is for example delaying the core collapse (Goodman \& Hut 1989). From a thermodynamic point of view, multi-star systems in globular clusters act as a heat source. The prerequisite for this mechanism is, of course, that globular clusters do contain multi-star systems at some point during their lifetime. For

* The full radial velocity sample (Table A.1) is only available at the CDS via anonymous ftp to cdsarc.u-strasbg. fr (130.79.128.5) or via http://cdsarc.u-strasbg.fr/viz-bin/cat/J/A+A/632/ A3 the main-sequence field stars $>50 \%$ (Duquennoy \& Mayor 1991 ; Duchêne \& Kraus 2013) in our Milky Way are in multi-star systems. In globular clusters, binary fractions are typically lower than in the field (Milone et al. 2012), but can reach values around $\sim 50 \%$ (e.g Sollima et al. 2007; Milone et al. 2012) in central regions. This radial gradient can be explained by mass segregation and indeed is reproduced in simulations (e.g. Fregeau et al. 2009; Hurley et al. 2007). The initial fraction of multi-star systems in globular clusters is changed due to dynamical formation and destruction processes caused by stellar encounters, as well as stellar evolution during the (typical long) life time of Galactic globular clusters. The primordial fraction of multi-star systems to single stars for globular clusters therefore cannot be easily inferred from observations (Hut et al. 1992). As a result, the initial fraction is poorly constrained and simulations use values in a wide range from 5\% (Hurley et al. 2007) to $100 \%$ (Ivanova et al. 2005). Current observations are still consistent with this wide range in primordial binary fractions, depending on which orbit distribution is assumed (Leigh et al. 2015). 
Until today there is only one technique with a high discovery efficiency for multi-star systems, namely high precision photometry (Sollima et al. 2007; Milone et al. 2012). It uses the fact that binary stars with both components contributing to the total brightness of one unresolved source have a position in the colour-magnitude-diagram (CMD) differing from single stars. For example, a large number of binaries is located to the brighter (redder) side of the main-sequence (MS). This method is sensitive to binaries in the MS with arbitrary orbital period and inclination. However, no constraints on orbital parameters are possible. The advantage is that this method is efficient in terms of observing time and produces large statistical robust samples. The disadvantage is that in terms of individual binary system properties it only has access to the biased mass-ratio parameter. Milone et al. (2012) investigated 59 Galactic globular clusters with this method using the Hubble Space Telescope (HST). They studied MS binaries with a mass ratio $q>0.5$ and found in nearly all globular clusters a significantly smaller binary frequency than $50 \%$. The results show a higher concentration of binaries in the core with a general decreasing binary fraction from the centre to about two core radii by a factor of $\sim 2$. A significant anti-correlation between the cluster binary frequency and its absolute luminosity (mass) was found. Additionally, the authors confirm a significant correlation between the fraction of binaries and the fraction of blue straggler stars (BSS), indicating a relation between the BSS formation mechanism and binaries.

In the literature there are almost no spectroscopic studies of the binarity in globular clusters. One systematic search was done in the globular cluster M4 based on 5973 spectra of 2469 stars by Sommariva et al. (2009). They discovered 57 binary star candidates and derived a lower limit of the total binary fraction of $(3.0 \pm 0.3) \%$ for M4.

In light of the important role of binaries in globular clusters, we designed the observing strategy of the MUSE globular cluster survey (Kamann et al. 2018) such that we can infer the binary fraction of every cluster in our sample. For all clusters we aim for at least three epochs of each pointing. For a selection of clusters, namely NGC 104 (47 Tuc), NGC 3201, and NGC 5139 ( $\omega$ Cen), we are also able to study individual binary systems in more detail. This paper concentrates on the globular cluster NGC 3201. NGC 3201 has, apart NGC 5139, the largest half-light and core radius of all globular clusters in our survey. Its binary fraction within the core radius appears to be relatively high $((12.8 \pm 0.8) \%$, Milone et al. 2012). In Giesers et al. (2018), we reported a quiescent detached stellar-mass black hole with $4.36 \pm 0.41) M_{\odot}$ in this cluster, the first black hole found by a blind spectroscopic survey. Based upon our discovery, NGC 3201 was predicted to harbour a significant population of black holes (see Kremer et al. 2018; Askar et al. 2018a). In our MUSE data, NGC 3201 has the deepest observations, the most epochs to date, and the least crowding. It is therefore the ideal case for our purpose.

This paper is structured as follows. In Sect. 2 we describe peculiar objects which are important in the context of binary star studies in NGC 3201. In Sect. 3 the observations, data reduction and selection of our final sample are discussed. In Sect. 4 we present a MOCCA simulation of the globular cluster NGC 3201 that we used to create a mock observation for comparisons and verifying the following method. We introduce a statistical method to determine the variability of radial velocity measurements and use the method to determine the binary fraction of NGC 3201 in Sect. 5. In Sect. 6 we explain our usage of the tool THE JOKER to find orbital solutions to individual binary systems and present their orbital parameter distributions. We discuss peculiar objects in our data, such as blue straggler stars, sub-subgiants and black hole candidates in Sect. 7. We end with the conclusions and outlook in Sect. 8.

\section{Peculiar objects in globular clusters}

\subsection{Blue straggler stars}

In most globular clusters a collection of blue straggler stars can be easily found in the CMD along an extension of the MS beyond the turn-off point. Their positions indicate more massive and younger stars than those of the MS. Without continuing star formation due to lacking gas and dust in globular clusters, mass transfer from a companion is the usual explanation for the formation of a blue straggler. This mass transfer could be on long timescales, like in compact binary systems, or on short timescales, as for collisions of two stars (in a binary system or of a priori not associated stars). However, it is still unclear which of these processes contribute to the formation of blue stragglers and how often. Ferraro et al. (1997) discovered a bimodal radial distribution of blue stragglers in M3 and suggested that the blue stragglers in the inner cluster are formed by stellar collisions and those in the outer cluster from merging primordial binaries. Sollima et al. (2008) identified that the strongest correlation in low-density globular clusters is between the number of blue stragglers and the binary frequency of the cluster. They suggested that the primordial binary fraction is one of the most important factors for producing blue stragglers and that the collisional channel to form blue stragglers has a very small efficiency in low-density globular clusters.

Hypki \& Giersz (2017) performed numerical simulations of globular clusters with various initial conditions. They found that the number of evolutionary blue stragglers (formed in a compact binary system) is not affected by the density of the globular cluster. In contrast, the number of dynamically created blue stragglers (by collisions between stars) correlates with the density. The efficiencies of both channels strongly depend on the initial semi-major axes distributions. Wider initial orbits lead to more dynamically created blue stragglers. Finally, they found in all globular cluster models at the end of the simulation a constant ratio between the number of blue stragglers in binaries and as single stars $R_{\mathrm{B} / \mathrm{S}} \sim 0.4$

A mass distribution of blue stragglers has been estimated by Fiorentino et al. (2014) from pulsation properties of blue stragglers in the globular cluster NGC 6541. They found a mass of $(1.06 \pm 0.09) M_{\odot}$, which is significantly in excess of the cluster MS turn-off mass $\left(0.75 M_{\odot}\right)$. Baldwin et al. (2016) found an average mass of blue straggler stars of $(1.22 \pm 0.12) M_{\odot}$ in 19 globular clusters. Geller \& Mathieu (2011) found a surprisingly narrow blue straggler companion mass distribution with a mean of $0.53 M_{\odot}$ in long-period binaries in the open cluster NGC 188. They suggested that this conclusively rules out a collisional origin in this cluster, as the collision hypothesis predicts significantly higher companion masses in their model.

\subsection{Sub-subgiant stars}

A star in a cluster is called a sub-subgiant (SSG) if its position in the CMD is redder than MS turn-off stars (and its binaries) and fainter than normal subgiant stars. These stars are related to the so called red stragglers (RS) which are redder than normal (red) giant stars. For our study this distinction is not important and we call them hereafter sub-subgiants. The evolution of these stars cannot be explained by single star evolution. 
B. Giesers et al.: Binaries in NGC 3201

Geller et al. (2017a) describe in detail the demographics of SSGs in open and globular clusters and conclude that half of their SSG sample are radial velocity binary stars with typical periods less than $15 \mathrm{~d}, 58 \%$ are $\mathrm{X}$-ray sources, and $33 \%$ are $\mathrm{H} \alpha$ emitters. The formation scenarios explained in Leiner et al. (2017) for SSG and RS are isolated binary evolution, the rapid stripping of a subgiant's envelope, or stellar collisions. So far, from observations and simulations, no channel can be excluded, but it seems that the binary channel is dominant in globular clusters (Geller et al. 2017b).

\subsection{SX Phoenicis-type stars}

SX Phoenicis-type (SXP) stars are low-luminosity pulsators in the classical instability strip with short periods. In globular clusters they appear as blue straggler stars with an enhanced helium content (Cohen \& Sarajedini 2012). SXP stars show a strong period-luminosity relation, from which distances can be measured. The period range is from $0.02 \mathrm{~d}$ to $0.4 \mathrm{~d}$ with typical periods around $\sim 0.04 \mathrm{~d} \approx 1 \mathrm{~h}$. SXP stars are thought to be formed in binary evolution, but it is not clear whether they are indicative of a particular blue straggler formation channel. The prototype star SX Phoenicis shows a radial velocity amplitude of $19 \mathrm{~km} \mathrm{~s}^{-1}$ due to radial pulsation modes (Kim et al. 1993).

\subsection{Black holes}

Observers are searching for two kinds of black holes in globular clusters. On the one hand, we have no conclusive evidence for central intermediate-mass black holes (IMBH, see Baumgardt 2017; Tremou et al. 2018). One of the challenges in searching for IMBHs is that typical signatures, such as a central cusp in the surface brightness profile or the velocity dispersion profile can also be explained by a binary star or stellar-mass black hole population (for Omega Cen, see Zocchi et al. 2019). On the other hand, there are known stellar-mass black holes in Galactic globular clusters (e.g. Strader et al. 2012; Giesers et al. 2018). If they are retained, stellar-mass black holes should quickly migrate to the cluster cores due to mass segregation, and strongly affect the evolution of their host globular clusters (e.g. Kremer et al. 2018; Arca Sedda et al. 2018; Askar et al. 2018a, 2019). However, the retention fraction of black holes in globular clusters is highly unknown, which also limits our abilities to interpret gravitational wave detections. For NGC 3201, Askar et al. (2018a) and Kremer et al. (2018) predicted that between 100 and more than 200 stellar-mass black holes should still be present in the cluster, with up to ten in a binary system with a MS star.

\section{Observations and data reduction}

The observational challenge in globular clusters is the crowded field resulting in multiple star blends. For photometric measurements of dense globular clusters, instruments such as the ones attached to the HST have just sufficient spatial resolution to get independent measurements of stars down the MS. In the past, spectroscopic measurements of individual globular cluster stars (with a reasonable spectroscopic resolution) were limited by crowding in the cluster centre. Since 2014 we are observing 27 Galactic globular clusters (PI: S. Kamann, formerly S. Dreizler) with the integral field spectrograph Multi Unit Spectroscopic Explorer (MUSE) at the Very Large Telescope (VLT; Kamann et al. 2018). In the wide field mode, MUSE covers a $1^{\prime} \times 1^{\prime}$ field of view (FoV) with a spatial sampling of $0.2^{\prime \prime}$ and a

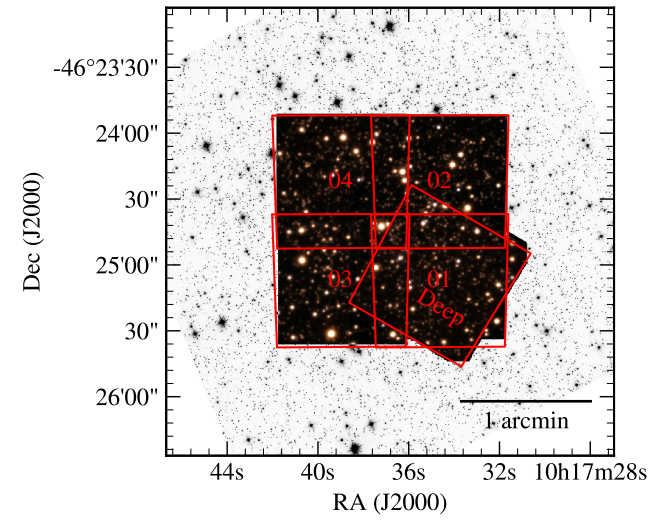

Fig. 1. Pointing chart of the MUSE instrument for the globular cluster NGC 3201. The inverted background image is from the ACS globular cluster survey (Sarajedini et al. 2007). The MUSE FoV is a collapsed RGB image created from the spectral cubes. Note the overlapping areas between the pointings.

spectral sampling of $1.25 \AA$ (resolving power of $1770<R<$ 3590 ) in the wavelength range from 4750 to $9350 \AA$. MUSE allows us to extract spectra of some thousand stars per exposure.

The overall aim of our stellar census in globular clusters with MUSE is twofold. On the one hand we investigate the spectroscopic properties of more than half a million of cluster members down to the MS. On the other hand we investigate the dynamical properties of globular clusters. Upcoming publications are in the field of multiple populations (Husser et al. 2019; Latour et al. 2019; Kamann et al., in prep.), the search for emission line objects (Göttgens et al. 2019a,b), cluster dynamics like the overall rotation (Kamann et al. 2018), and gas clouds in direction of the clusters (Wendt et al. 2017). Since this survey represents the first blind spectroscopic survey of globular cluster cores, we expect many unforeseen discoveries.

For this paper, we used all observations of NGC 3201 obtained before May 2019. The pointing scheme can be seen in Fig. 1. In addition to the pointings listed in Kamann et al. (2018), we also implemented a deep field mainly to go deeper on the MS. Table 1 lists the number of visits and total integration times for the different pointings. Our data include adaptive optics (AO) observations, our standard observing mode since the AO system was commissioned in October 2017. The AO observations are treated in the same way as our previous observations. The only difference is that a wavelength window of each spectrum around the sodium lines ( $5805 \AA-5965 \AA$ ) has to be masked due to the AO laser emission. During each visit, the pointing was observed with three different instrument derotator angles $(0,90$, $180^{1}$ degrees) in order to reduce systematic effects of the individual MUSE spectrographs.

Figure 1 shows our observation scheme for NGC 3201. We secured at least 12 observations per pointing (see Table 1 and Fig. 2). Thanks to the overlapping pointings, up to 63 independent observations per star are available in the central region (see Fig. 3).

To differentiate between radial velocity signals from short period binaries and those of longer period binaries, we observed each of the four pointings twice with a separation of two to three

1 The deep pointing was observed with an additional derotator angle of 270 degrees. 
Chapter 2. Publications

A\&A 632, A3 (2019)

Table 1. Exposures, observations, and integration time per pointing.

\begin{tabular}{cccc}
\hline \hline Pointing & Exposures & Observations & Total time [min.] \\
\hline 01 & 49 & 17 & 163 \\
02 & 49 & 15 & 143 \\
03 & 44 & 15 & 143 \\
04 & 36 & 12 & 114 \\
Deep & 16 & 4 & 160 \\
\hline$\sum$ & 194 & 63 & 723 \\
\hline
\end{tabular}
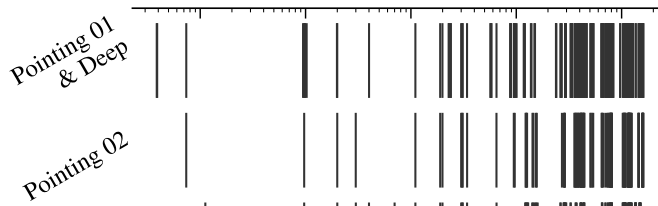

pointion<smiles>COCCO</smiles>

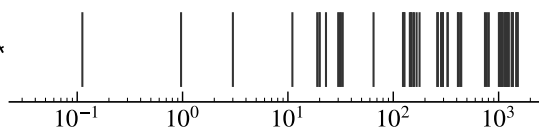

$\Delta t$ [days]

Fig. 2. All possible measurement baselines per star and pointing. $\Delta t$ is the time between two observations.

hours in a single night. We already had this distinction sometimes for the stars observed within the same night in the overlapping region of two pointings (like for the published black hole candidate). Without that short baseline a hard binary with a period below one day could mimic the signal of a companion of a black hole candidate at a longer period (temporal aliasing).

The reduction of all exposures was carried out using the standard MUSE pipeline (Weilbacher et al. 2012, 2014). The extraction from the final product of each visit (epoch) is done by a PSF-fitting technique implemented in the software PAMPELMUSE (Kamann et al. 2013). After an extraction is finished the individual spectra are fitted against a synthetic stellar library to determine stellar properties with SPEXXY (Husser et al. 2013). The extraction and stellar parameter fitting methods are summarised in Husser et al. (2016) and Kamann et al. (2018).

\subsection{Radial velocities}

In this study, we want to investigate if a given star is in a binary system using the radial velocity method. Binaries that are able to survive in the dense environment of a globular cluster are so tight, that even HST is not able to spatially resolve the binary components at the distance of NGC 3201. For this reason, the binary system will appear as a single point-like source. Except for the case when both stars have similar brightness (see Sect. 4 on how we handle these binaries), the extracted MUSE spectrum will be dominated by one star. The measured radial velocity variation of this star can be used to determine the properties of the binary system indirectly.

Since we aim for the highest possible radial velocity precision, we checked if the standard wavelength calibration of the MUSE instrument and the uncertainties calculated by the extraction and fitting routines are reliable. For this purpose we analysed the wavelength shift of the telluric lines in the extracted

A3, page 4 of 20

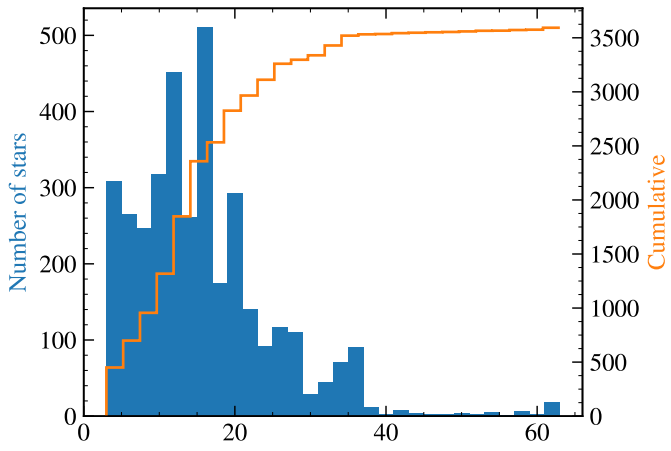

Number of applicable observations per star

Fig. 3. Histogram of applicable (accepted after filtering) number of observations per star (blue) and cumulative distribution of the same data (orange).

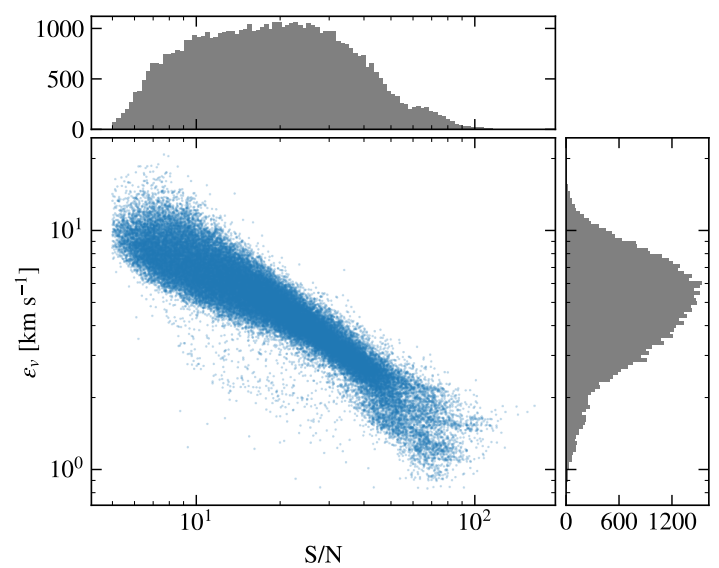

Fig. 4. Radial velocity uncertainties $\epsilon_{v}$ of all spectra in the sample used for NGC 3201 as a function of the spectral signal-to-noise.

spectra per observation to correct for tiny deviations in the wavelength solution of the pipeline (see Sect. 4.2 in Kamann et al. 2018 , for details). We also cross checked the radial velocity of every spectrum obtained by the full-spectrum fit using a crosscorrelation of the spectrum with a PHOENIX-library template (Husser et al. 2013) (see criterion in Sect. 3.2). Figure 4 shows the resulting uncertainties $\epsilon_{v}$ as a function of $S / N$ of the spectra in our final sample of NGC 3201. We get reliable radial velocities from $S / N>5$ spectra (Kamann et al. 2018). At $S / N=10$ the uncertainties are $\epsilon_{v} \approx(7.0 \pm 1.7) \mathrm{km} \mathrm{s}^{-1}$, at $S / N \gtrsim 50$ the uncertainties are $\epsilon_{v} \approx(1.7 \pm 0.5) \mathrm{km} \mathrm{s}^{-1}$. These uncertainties represent our spectroscopic radial velocity precision, the accuracy of the MUSE instrument (wavelength shift between two observations) is below $1 \mathrm{~km} \mathrm{~s}^{-1}$ (Kamann et al. 2018).

\subsection{Selection of the final sample}

For the sample selection two things are crucial. Firstly, we only want reliable radial velocities with Gaussian distributed uncertainties. Secondly, our sample should only consist of single and multiple star systems which are cluster members, excluding 
Galactic field stars. Finally, stars that mimic radial velocity variations due to intrinsic pulsations should be excluded. To ensure this the following filters are applied:

- The reliability of every single spectrum is ensured by the subsequent criteria, which are either 0 (false) and 1 (true). We apply a cut for the signal to noise $S / N$ of a spectrum

$R_{S / N}=S / N \geq 5$.

The quality of the cross-correlation is checked using the $r$-statistics and FWHM defined by Tonry \& Davis (1979)

$R_{\mathrm{cc}}=r \geq 4 \wedge F W H M>10 \AA$.

A plausible uncertainty $\epsilon_{\mathrm{v}, \mathrm{cc}}$ of the cross-correlation radial velocity $v_{\mathrm{cc}}$

$R_{\epsilon_{\mathrm{v}, \mathrm{cc}}}=\epsilon_{\mathrm{v}, \mathrm{cc}}>0.1 \mathrm{~km} \mathrm{~s}^{-1}$,

and a plausible uncertainty $\epsilon_{\mathrm{v}}$ of the full-spectrum fit radial velocity $v$

$R_{\epsilon_{\mathrm{v}}}=\epsilon_{\mathrm{v}}>0.1 \mathrm{~km} \mathrm{~s}^{-1}$

is ensured. We check that the velocity is within $3 \sigma$ of the cluster velocity $v_{\text {cluster }}$ and the cluster dispersion $\sigma_{\mathrm{v} \text {,cluster }}$ including the standard deviation taken from the Harris (1996, 2010 edition) catalogue. For typical binary amplitudes we add $30 \mathrm{~km} \mathrm{~s}^{-1}$ to the tolerance, yielding

$$
R_{v}=\frac{\left|v-v_{\text {cluster }}\right|}{\sqrt{\epsilon_{\mathrm{v}}^{2}+\sigma_{\mathrm{v}, \text { cluster }}^{2}+\left(30 \mathrm{~km} \mathrm{~s}^{-1}\right)^{2}}} \leq 3 .
$$

The last criterion is the check if cross-correlation and fullspectrum fit are compatible within $3 \sigma$ with each other,

$R_{v=v_{\mathrm{cc}}}=\frac{\left|v-v_{\mathrm{cc}}\right|}{\sqrt{\epsilon_{\mathrm{V}}^{2}+\epsilon_{\mathrm{v}, \mathrm{cc}}^{2}}} \leq 3$.

We combine all these criteria in an empirically weighted equation, which gives us a result between 0 and 1 ,

$R_{\text {total }}=\left(2 R_{S / N}+10 R_{\mathrm{cc}}+R_{\epsilon_{\mathrm{v}, \mathrm{cc}}}+3 R_{v}+2 R_{\epsilon_{\mathrm{v}}}+5 R_{v=v_{\mathrm{cc}}}\right) / 23$.

A radial velocity is considered as reliable if the reliability $R_{\text {total }}$ surpasses $80 \%$.

- We exclude spectra which are extracted within 5 px from the edge of a MUSE cube to avoid systematic effects.

- We exclude spectra where PAMPELMUSE was unable to deblend multiple nearby HST/ACS sources (this is of course seeing dependent and affects mostly faint sources).

- To identify confusion or extraction problems in a MUSE spectrum, we calculated broad-band magnitudes from the spectrum in the same passband that was used in the extraction process and calculated the differences between input and recovered magnitudes. We accept only spectra with a magnitude accuracy $>80 \%$ (see Sect. 4.4 in Kamann et al. 2018, for more details).

- In some low $S / N$ cases the cross-correlation and fullspectrum fit returned similar but wrong radial velocities due to noise in the spectrum. These could be outliers by several $100 \mathrm{~km} \mathrm{~s}^{-1}$ introducing a strong variation in the signal and passing the reliability criteria described before. To find outliers we generally compare all radial velocities $v_{i}$ (with their uncertainties $\left.\epsilon_{v, i}\right)$ of a single star with each other. We define the set of all radia velocities as $A=\left\{v_{i}\right\}$. An outlier $v_{x}$ in the subset $B=A \backslash\left\{v_{x}\right\}$ of all radial velocities except the inspected radial velocity, is identified with

$\frac{\sigma_{A}}{\sigma_{B}}>\kappa \wedge \frac{\left|v_{x}-\tilde{B}\right|}{\epsilon_{v, x}+\sigma_{B}}>\kappa \quad$ with $\kappa=3$.

$\tilde{B}$ is the median of the radial velocities $v_{i}$ in the set $B . \sigma$ is the standard deviation of the corresponding quantity. Using this equation outliers are identified and excluded from further analyses.

- We manually set the binary probability in our sample to 0 for 10 RR Lyrae- and 5 SX Phoenicis-type stars known in the Catalogue of Variable Stars in Galactic Globular Clusters (Clement et al. 2001; Clement 2017) and Arellano Ferro et al. $(2014)^{2}$.

- Since NGC 3201 has a heliocentric radial velocity of $(494.0 \pm 0.2) \mathrm{km} \mathrm{s}^{-1}$ and a central velocity dispersion of $(5.0 \pm$ $0.2) \mathrm{km} \mathrm{s}^{-1}$ (Harris 1996, 2010 edition), we select members by choosing stars with mean radial velocities $>400 \mathrm{~km} \mathrm{~s}^{-1}$.

- Our final sample only consists of member stars with at least 3 spectra per star after all filters have been applied.

We end up with 3553 stars and 54883 spectra out of 4517 stars and 68084 spectra in total. Due to different observational conditions the stars near the confusion limit cannot be extracted reliably from every observation. Figure 3 shows a histogram of all applicable observations per star for the final sample of NGC 3201. We have 3285 (91\%) stars with five or more epochs, $2637(73 \%)$ with ten or more epochs, and $918(26 \%)$ stars with 20 or more epochs.

\subsection{Mass estimation}

We estimate the mass of each star photometrically by comparing its colour and magnitude from the ACS globular cluster survey (Sarajedini et al. 2007; Anderson et al. 2008) with a PARSEC isochrone (Bressan et al. 2012). For the globular cluster NGC 3201, we found the best matching isochrone compared to the whole ACS CMD with the isochrone parameters $[\mathrm{M} / \mathrm{H}]=-1.39$ dex (slightly above the comparable literature value $[\mathrm{Fe} / \mathrm{H}]=-1.59 \mathrm{dex}$, Harris 1996, 2010 edition), age $=11 \mathrm{Gyr}$, extinction $E_{B-V}=0.26$, and distance $=4.8 \mathrm{kpc}$. The mass for each star is determined from its nearest neighbour on the isochrone. For blue straggler stars we assume a mass of $(1.20 \pm 0.05) M_{\odot}($ Baldwin et al. 2016) instead.

\subsection{The electronic catalogue}

An outline of the final sample of the radial velocities is listed in Table A.1. The columns ACS Id, position (RA, Dec), and $\mathrm{V}$ equivalent magnitude from the ACS catalogue (Sarajedini et al. 2007) are followed by the mean observation date of the combined exposures (BMJD), the barycentric corrected radial velocity $v_{\mathrm{r}}$, and its uncertainty $\epsilon_{v}$ from our MUSE observations. The last column contains the probability for variability in radial velocity $P\left(\chi_{i}^{2}, v_{i}\right)$, determined as described in Sect. 5 . Three versions of the catalogue, one with the full final sample (see Sect. 3.2), one with non-member stars, and one with the

2 The RR Lyrae-type stars are the brightest stars showing radial velocity variations in our sample and we verified that some of them show Keplerian-like signals with periods $<1 \mathrm{~d}$ and high eccentricities. 
Chapter 2. Publications

A\&A 632, A3 (2019)

Table 2. Globular cluster properties for the MOCCA model in comparison to observed values.

\begin{tabular}{lll}
\hline \hline RA & $10^{\mathrm{h}} 17^{\mathrm{m}} 36^{\mathrm{s}} 82^{(a)}$ \\
Dec & $-46^{\circ} 24^{\prime} 444^{\prime \prime} 9^{(a)}$ \\
Distance to Sun & $4.9 \mathrm{kpc}^{(a)}$ \\
Metallicity [Fe/H] & $-1.59 \mathrm{dex}$ \\
Barycentric radial velocity & $(494.0 \pm 0.2) \mathrm{km} \mathrm{s}^{-1(a)}$ \\
\hline & MOCCA & Literature $^{(a)}$ \\
\hline Central velocity dispersion $\left[\mathrm{km} \mathrm{s}^{-1}\right]$ & $5.23^{(*)}$ & $(5.0 \pm 0.2)^{(a)}$ \\
Core radius [pc] & 1.54 & $1.85^{(a)}$ \\
Half-light radius [pc] & 4.16 & $4.42^{(a)}$ \\
Total $V$-band luminosity $\left[L_{\odot}\right]$ & $9.27 \times 10^{4}$ & $8.17 \times 10^{4(a)}$ \\
Central surface brightness $\left[L_{\odot} / \mathrm{pc}^{2}\right]$ & $2.25 \times 10^{3}$ & $9.14 \times 10^{2(a)}$ \\
Age [Gyr] & 12 & $(12.0 \pm 0.8)^{(b)}$ \\
Mass $\left[M_{\odot}\right]$ & $2.3 \times 10^{5}$ & $(1.49 \pm 0.09) \times 10^{5(c)}$ \\
Total binary fraction $[\%]$ & 8.72 & - \\
\hline
\end{tabular}

Notes. ${ }^{(*)}$ Computed for stars brighter than 3 mags below MS turn-off References. ${ }^{\left({ }^{a}\right)}$ Harris $\left(1996,2010\right.$ edition) catalogue; ${ }^{(b)}$ Dotter et al. (2010); ${ }^{(c)}$ Baumgardt \& Hilker (2018).

RR Lyrae and SX Phoenicis stars are available at the CDS and on the project website ${ }^{3}$.

\section{MOCCA simulation of NGC 3201}

We want to compare our observations of binaries in NGC 3201 with models. Our first attempt using a toy model with realistic binary parameter distributions for this specific cluster like of period, eccentricity, mass ratio, etc. failed since the creation of binaries from these distributions is unrealistic. Moe \& Di Stefano (2017) demonstrated that randomly drawing values of these orbital parameter distributions does not lead to realistic currently observed binary distributions even when the parameter distributions themselves are realistic. Especially not for globular clusters where dynamical interactions between stars play an important role. Thus we have to use more sophisticated simulations of globular clusters including all relevant physics for the cluster dynamics and the binary evolution. Simulating the dynamical evolution of realistic globular clusters in a Galactic potential up to a Hubble time is computationally expensive.

\subsection{The mode}

An approach that has been used to extensively simulate realis tic globular cluster models is the Monte Carlo method for stellar dynamics that was first developed by Hénon (1971). This method combines the statistical treatment of relaxation with the particle based approach of direct $N$-body methods to simulate the long term evolution of spherically symmetric star clusters. The particle approach of this method enables the implementation of additional physical processes (including stellar and binary evolution) and is much faster than direct $N$-body methods. In recent years, an extensive number of globular cluster models have been simulated with the MOCCA (MOnte Carlo Cluster simulAtor; Giers 1998; Hypki \& Giersz 2013; Giersz et al. 2013; Askar et al. 2017; Hong et al. 2018; Belloni et al. 2019) and CMC (Clus ter Monte Carlo) codes (Joshi et al. 2000; Pattabiraman et al 2013; Morscher et al. 2015; Rodriguez et al. 2016; Chatterjee et al. 2017)

For the purpose of this project we needed a simulated globular cluster model that would have present-day observational properties comparable to NGC 3201 and that would also contain

3 https://musegc.uni-goettingen.de/

A3, page 6 of 20 stellar-mass black holes like the one we found (Giesers et al. 2018). Therefore, we used results from the MOCCA Survey Database I (Askar et al. 2017) to identify initial conditions for a model that had global properties close to NGC 3201 at an age of 12 Gyr. This model has been subsequently refined with additional MOCCA simulations based on slightly modified initial conditions to find an even better agreement with the properties of NGC 3201 at an age of 12 Gyr including total luminosity, core and half-light radii, central surface brightness, velocity dispersion and binary fraction.

The MOCCA code utilizes the Monte Carlo method to com pute relaxation. Additionally, it uses the fewbody code (Fregeau et al. 2004, small number N-body integrator) to compute the outcome of strong interactions involving binary stars. MOCCA also makes use of the SSE (Hurley et al. 2000) and BSE (Hurley et al. 2002) codes to carry out stellar and binary evolution of all stars in the cluster. MOCCA results have been extensively compared with results from direct $N$-body simulations and they show good agreement for both the evolution of global properties and number of specific objects (Giersz et al. 2013; Wang et al. 2016)

The following initial parameters were used for the MOCCA model we simulated specifically for this work to have a comparison to NGC 3201: The initial number of objects was set to $N=5.5 \times 10^{5}$. The initial binary fraction was set to $50 \%$, resulting in $2.25 \times 10^{5}$ single stars and $5.5 \times 10^{5}$ stars in binary systems, thus in total $8.25 \times 10^{5}$ stars in the initial model. The initia binary properties were set as described in Belloni et al. (2017). The metallicity of the model was set to $\left(Z=5.1 \times 10^{-4}\right)$ to correspond to the observed metallicity of NGC 3201 provided in the Harris (1996, 2010 edition) catalogue. We used a King (1966) model with a central concentration parameter $\left(W_{0}\right)$ of 6.5 . The model had an initial half-mass radius of $1.65 \mathrm{pc}$ and a tidal radius of $165 \mathrm{pc}$. The initial stellar masses were sampled using a two-component Kroupa (2001) initial mass function (IMF) with masses in the range $0.08 M_{\odot}$ to $100 M_{\odot}$. Neutron star natal kicks were drawn from the Hobbs et al. (2005) distribution. For black holes, natal kicks were modified according to black hole masses that were computed following Belczynski et al. (2002). The properties of the evolved simulated model at $12 \mathrm{Gyr}$ are listed in Table 2 and compared to literature values. The MOCCA model for NGC 3201 is able to reproduce well the observational core radius, half-ligh radius, central velocity dispersion, and the $V$-band luminosity provided in Harris (1996, 2010 edition). The central surface brightness for the MOCCA model is a factor of 2.5 larger than the observed one which is unfortunately not given with an uncertainty. In contrast, McLaughlin \& van der Marel (2005), for example, found about twice the observational central surface brightness listed in Harris (1996, 2010 edition). Given the observational uncertainties and limitations, the agreement between the simulated model and the observed values are reasonable.

\subsection{The mock observation}

We project the simulation snapshot at $12 \mathrm{Gyr}$ in Cartesian coordinates using the COCOA code (Askar et al. 2018b). We use the distance modulus of NGC 3201 at a distance of $4.9 \mathrm{kpc}$ (Harris 1996, 2010 edition) to get the apparent magnitude of all stars For binary stars we combine the two components into a single magnitude. We select the stars within the MUSE FoV (of all pointings). Additionally we use our observational completeness function for NGC 3201 (see Fig. 5) to select stars accordingly.

After we identified a set of stars comparable to the MUSE observations, we created mock radial velocities for these stars. To this aim, we let our observational data guide the simulation. For 


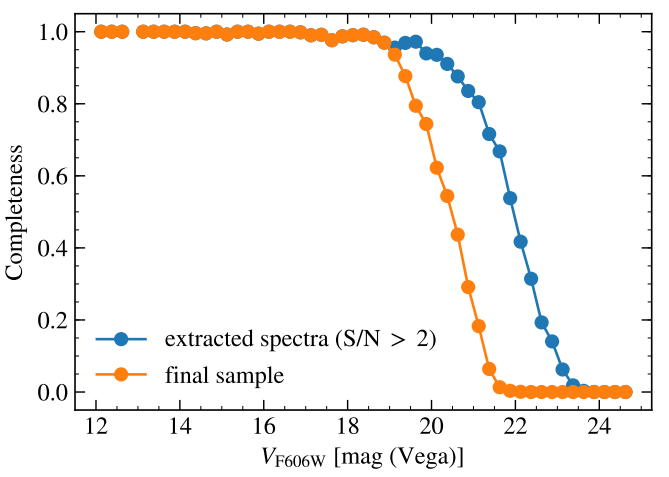

Fig. 5. Completeness as a function of magnitude for all MUSE observations of NGC 3201, using photometry from the ACS globular cluster survey (Sarajedini et al. 2007). The fraction of all possible extracted spectra to observed spectra within the MUSE FoV is shown. Blue: extracted spectra with $S / N>2$. Orange: extracted spectra in the final sample (see Sect. 3.2). (The uncertainties are smaller than the points.)

each observed star (in our MUSE sample) we randomly picked a MOCCA star with comparable magnitude. We then used the epochs of the observed star to create new radial velocity measurements. For single stars we simply assumed a radial velocity of $0 \mathrm{~km} \mathrm{~s}^{-1}$ for every time step. For binaries we calculated the radial velocity for the brighter component from a Keplerian orbit using the MOCCA properties of the binary system (masses, period, inclination, eccentricity, argument of periastron, and periastron time). However, due to the spectral resolution of MUSE its measured radial velocity amplitude will be influenced by the flux ratio of the two components of the binary system. From Monte Carlo simulations we found that the theoretical expected radial velocities $v_{\mathrm{r} \text {,theoretical }}$ are linearly damped with the flux ratio:

$v_{\mathrm{r} \text {, measured }}=v_{\mathrm{r}, \text { barycentre }}+\left(1-\frac{f_{2}}{f_{1}}\right) v_{\mathrm{r}, \text { theoretical }}$.

With the flux of the brighter component $f_{1}$ and the fainter component $f_{2} \cdot v_{\mathrm{r} \text {,barycentre }}$ is the radial velocity of the barycentre, which can be neglected if only relative velocities are of interest. In the case of both components having the same flux, the measured radial velocity $v_{\text {r,measured }}$ amplitude will be zero. Thus, for example, twin MS binary stars are hard to find. In the case when the fluxes of the components are very different, the observed radial velocity amplitude corresponds to the amplitude of the brighter component. We apply this damping for every simulated radial velocity. Finally we scatter these radial velocities within the uncertainties taken from the MUSE corresponding measurements.

\section{The binary fraction of NGC 3201}

Section 4 reflects the efforts required to create realistic models for globular clusters. We therefore conceived a model independent approach to derive the binary frequency of a globular cluster, as well as the probability of an individual star to be radial velocity variable.

\subsection{A new method to detect variable stars}

We here introduce a general statistical method which could be applied to any inhomogeneous sample with a varying number of (a few) measurements and a large range of uncertainties. Here it is applied to radial velocity measurements: To detect radial velocity variations in a given star with $m$ measurements, we compute the $\chi_{i}^{2}$ for the set of measurements $x_{j}$ with uncertainties $\sigma_{j}$ to determine how compatible they are with the constant weighted mean $\bar{x}$ (null hypothesis) of the measurements:

$\chi_{i}^{2}=\sum_{j=1}^{m} \frac{1}{\sigma_{j}^{2}}\left(x_{j}-\bar{x}\right)^{2} \quad$ with $\quad \bar{x}=\frac{\sum_{j=1}^{m} \frac{x_{j}}{\sigma_{j}^{2}}}{\sum_{j=1}^{m} \frac{1}{\sigma_{j}^{2}}}$.

A star that shows radial velocity variations higher than the associated uncertainties will have reduced $\chi^{2}>1$ on average. In contrast, a star without significant variations will have reduced $\chi^{2} \approx 1$. As described in Sect. 3 each star in our sample has its own number of observations and therefore its own number of degrees of freedom ( $v=m-1$, see Fig. 3). Assuming Gaussian distributed uncertainties for all measurements and a common degree of freedom for all stars, we know the expected $\chi^{2}$-distribution in form of the cumulative distribution function (CDF) $F$ for the null hypothesis that all stars show constant signals. If there are binary stars with radial velocity variations in our sample, there will be an excess of higher $\chi^{2}$ values compared to the null hypothesis. Therefore, the empirical CDF computed from the measured $\chi^{2}$ will increase slower than the CDF computed using the null hypothesis.

For a chosen degree of freedom (e.g. $v=3$ ) we calculate the probability $\bar{P}$ of each star to match the null hypothesis through the comparison of the observed empirical distribution function (EDF) $F_{\text {observed }}$ with the expected CDF (comparable to the top panel in Fig. 6):

$\bar{P}\left(\chi_{i}^{2}, v_{i}=3\right)=\frac{1-F\left(\chi_{i}^{2}, 3\right)_{\text {theoretical }}}{1-F\left(\chi_{i}^{2}, 3\right)_{\text {observed }}}$.

Since the EDF $F_{\text {observed }}$ is simply the fraction of stars below a given $\chi^{2}$, we divide the number of stars we measured below a given $\chi^{2}$ with the expected number from the known CDF to calculate $P$ (comparable to the middle panel in Fig. 6). The probability of a star to be variable for a given degree of freedom $v$ is $P=1-\bar{P}$ :

$P\left(\chi_{i}^{2}, v_{i}\right)=\frac{F\left(\chi_{i}^{2}, v_{i}\right)_{\text {theoretical }}-F\left(\chi_{i}^{2}, v_{i}\right)_{\text {observed }}}{1-F\left(\chi_{i}^{2}, v_{i}\right)_{\text {observed }}}$.

To use the statistical power of the whole sample with the total number of stars $n$, this equation can be generalised to multiple degrees of freedom by noticing that $n F_{\text {theoretical }}$ is the expected number of stars below a given $\chi^{2}$. The (expected or observed) total number of stars below a given $\chi^{2}$, taking all available degrees of freedom into account, can be expressed using the number of the stars per degree of freedom $n_{v}$ and adding up the contributions from all degrees of freedom in a "super CDF":

$\widehat{S}\left(\chi_{i}^{2}\right)=\frac{1}{n} \sum_{v} F\left(\chi_{i}^{2}, v\right) n_{v}$,

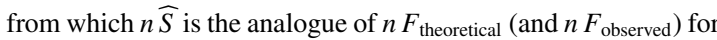
multiple degrees of freedom. Inserting this into Eq. (4) yields

$P\left(\chi_{i}^{2}, v_{i}\right)=\frac{n \widehat{S}\left(\chi_{k}^{2}\right)-k}{n-k} \quad$ with $k=\left|\left\{\chi^{2} \mid \chi^{2} / v<\chi_{i}^{2} / v_{i}\right\}\right|$

where $k$ is the number of stars with a reduced $\chi^{2}$ lower than the one of the given star, i.e. $\chi_{i}^{2} / v_{i}$ and $\chi_{k}^{2}$. 

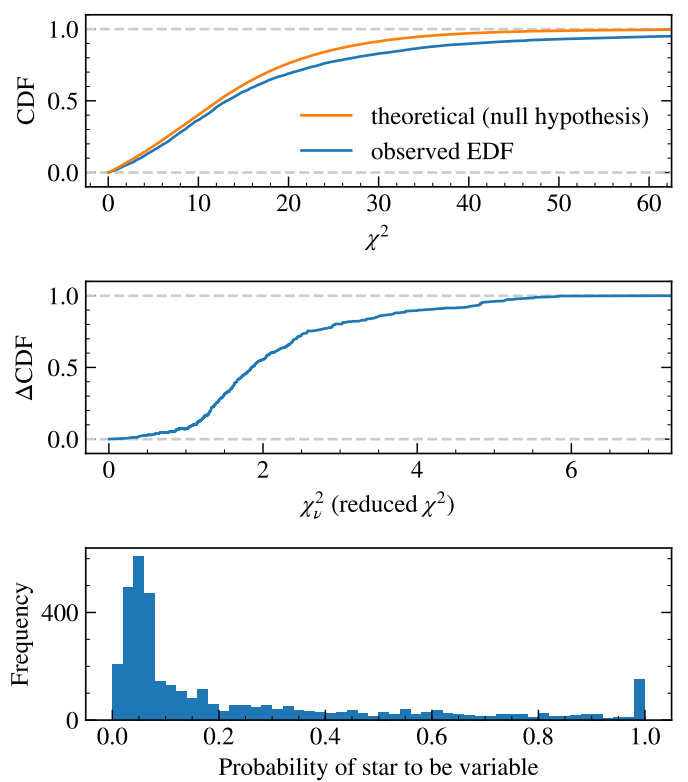

Fig. 6. Top panel: superposition of observed empirical distribution function (EDF) and expected theoretical cumulative distribution function (CDF) using the null hypothesis that no variable star exist in sample. Middle panel: resulting $\triangle \mathrm{CDF}$. Bottom panel: histogram of variability probabilities in the sample.

The upper panel of Fig. 6 shows the observed EDF and the theoretical "super CDF" $\widehat{S}$. Both functions deviate for increasing $\chi^{2}$, which indicates that stars in the sample not only show statistical variations. The middle panel shows the resulting $\triangle \mathrm{CDF}$ (Eq. (6)) for our sample. If we evaluate it with the reduced $\chi_{v}^{2}$ we get the probability for each star to vary in radial velocity. The bottom panel of Fig. 6 shows the resulting binary probability distribution of all NGC 3201 stars in our sample.

Comparing the number of stars with $P>0.5$ to the total number of stars yields the discovery binary fraction. We checked this approach using Monte Carlo simulations for different star samples of single and binary stars. It should be noted that at this 50\% threshold there is a balance of false-positives and false-negatives, but that ensures a robust measurement for the overall statistics. Of course, for individual stars the acceptance probability to be a binary should be higher.

The statistical uncertainty of the binary fraction is calculated by the quadratic propagation of the uncertainty determined by bootstrapping (random sampling with replacement) the sample and the difference of the fraction for $P>0.45$ and $P>0.55$ divided by 2 as a proxy for the discriminability uncertainty between binary and single stars.

\subsection{Verification of the method on the MOCCA mock observation}

To verify the validity of this statistical method, we applied it to the MOCCA mock observation introduced in Sect. 4. For each star we calculated the variability probability according to Eq. (6). We also calculated a kind of semi-amplitude by bisection of the peak to peak of the simulated radial velocities $\left(\Delta v_{r} / 2\right)$ of each
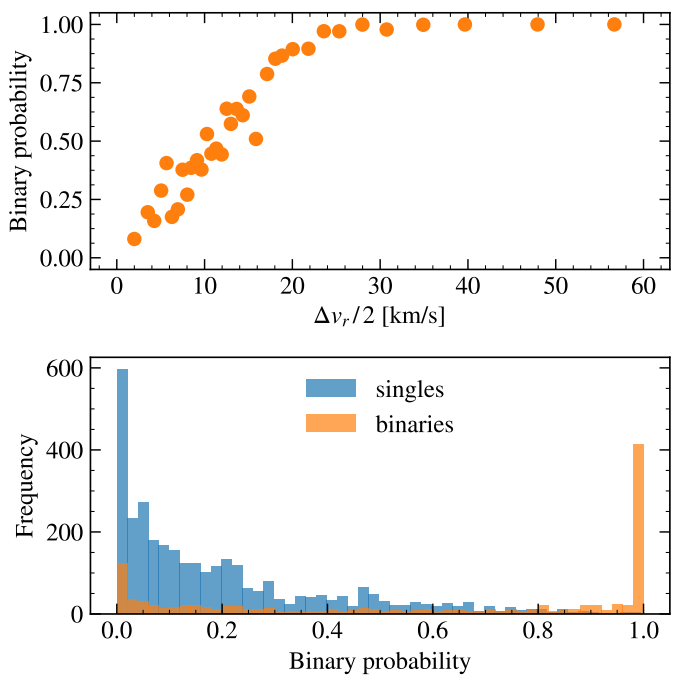

Fig. 7. Top panel: variability probability as a result of the statistica method for all known binaries in the MOCCA mock observation as a function of $\Delta v_{r} / 2$, a proxy for the projected radial velocity semiamplitude. Bottom panel: histograms of the variability probability for known single and binary stars in the MOCCA mock observation.

star. In the top panel of Fig. 7 the mean binary probability in relation to this semi-amplitude for all known binaries in the mock observation is presented, using a binning of 30 stars per data point. In view of the radial velocity uncertainties (see Fig. 4) the statistical method gives plausible results: for example $50 \%$ of all stars with a semi-amplitude around $10 \mathrm{~km} \mathrm{~s}^{-1}$ are recovered. The bottom panel of Fig. 7 shows histograms of the binary probability of the known single stars (in blue) and the known binary stars (in orange). We get a separation of the binary stars (peak at probability $\approx 1$ ) and single stars (peak at probability $\approx 0$ ). It shows the power of our approach, as it results in a binary distribution peaked at high probabilities and a single star distribution peaked at low probabilities. Using a threshold of $P=0.5(0.8)$ would result in a false-positive rate of 324/781 (44/617) stars. These rates agree with the expectations, since the method gives us a balance of false-positives and false-negatives at the 50\% threshold, as mentioned before.

The statistical method yields a discovery fraction of $(22.1 \pm$ $1.8) \%$ for this mock observation. Compared with the true binary fraction in this mock observation of $25.9 \%$ we get a discovery efficiency of $85.2 \%$. This implies that we can expect to detect the vast majority of binary stars present in our observed MUSE sample.

\subsection{Application to the MUSE observations}

Using the statistical approach we obtained an observational binary frequency (discovery fraction) of $(17.1 \pm 1.9) \%$ within our MUSE FoV. The bottom panel of Fig. 6 shows the resulting binary probability of our stars in the sample. To determine the true binary frequency of the globular cluster NGC 3201 we have to overcome our observational biases. Our survey is a blind survey and we do not select our targets as in previous spectroscopic studies. Nevertheless, we still have a magnitude limit below which we cannot derive reliable radial velocities and we 
are confined to the MUSE FoV. In Fig. 5 the magnitude completeness of MUSE stars compared to the ACS globular cluster survey (Sarajedini et al. 2007) of NGC 3201 is shown (blue curve). After filtering (see Sect. 3), we end up with the magnitude completeness of the binary survey (orange curve). To get the binary frequency we have to divide the discovery fraction by the discovery efficiency. As described in Sect. 4, the discovery efficiency cannot be derived by simple assumptions (except for inclination of the binary system). Moreover, the efficiency strongly depends on the number of observations and the sampling per star. Figure 3 shows that our distribution of applicable observations is inhomogeneous and likewise is our time sampling (see Fig. 2)

We use the MOCCA mock observation to overcome these biases. Assuming the MOCCA simulation is realistic and comparable to our MUSE observation, we found a binary frequency of $(20.08 \pm 0.22) \%$ within our MUSE FoV, taking the discovery efficiency of Sect. 5.2 into account. We can now use the original MOCCA simulation and compare its true binary frequency of $8.72 \%$ with the true binary frequency of the mock observation $25.9 \%$ and get a factor of 0.336 . Applying this factor and the discovery efficiency it is possible to translate the MUSE discovery fraction into the total binary fraction of NGC 3201 to

$(6.75 \pm 0.72) \%$.

The main reason for this value being significantly lower than our observed binary frequency is the central increase in binaries due to mass segregation. Our value is in reasonable agreement with the binary fraction of the MOCCA simulation, $8.72 \%$, considering the assumptions that have to be made in Monte Carlo models. This highlights again that the MOCCA simulation that we selected represents an accurate model for NGC 3201.

We also verified to what extent our study is consistent with the results of Milone et al. (2012), who reported a core binary frequency of $(12.8 \pm 0.8) \%$. Without applying any selection function, the MOCCA simulation yields a binary fraction of $12.5 \%$ within the core radius of NGC 3201. Hence, our results appear to be consistent with the study of Milone et al. (2012) and the apparent differences (with respect to our discovery fraction of $(20.08 \pm 0.22) \%)$ can be attributed to selection effects.

\subsection{Primordial binaries}

We created different MOCCA simulations of NGC 3201 to get a comparable mock observation and thus binary fraction to our observations. The best matching MOCCA simulation (see Sect. 4) has an initial binary fraction of 50\% which indicates that a large portion of primordial binaries is necessary to reproduce the current observations of NGC 3201.

Recent studies have shown that populations of cataclysmic variables and X-ray sources observed in several globular clusters can be better reproduced with globular cluster simulations that assume high primordial binary fractions (e.g. Rivera Sandoval et al. 2018; Cheng et al. 2018; Belloni et al. 2019). In addition, Leigh et al. (2015) and Cheng et al. (2019) found that a high primordial binary fraction is also necessary to reproduce the binary fraction outside the half-mass radius and the mass segregation we observe in Galactic globular clusters.

\subsection{Mass segregation}

Due to mass segregation it is expected that binary systems (which are in total more massive than single stars) will migrate towards the centre of a globular cluster (see Sect. 1). Thus the binary fraction should increase towards the cluster centre. In

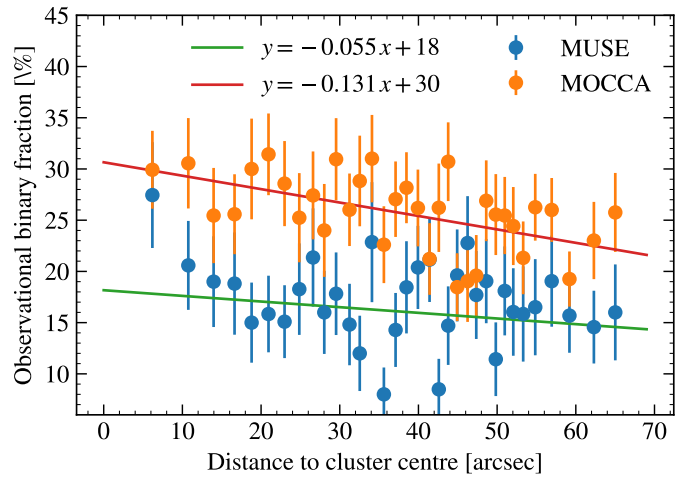

Fig. 8. Observational binary fraction of NGC 3201 in radial bins as a function of the cluster centre. Blue: MUSE bins with 100 stars per bin. Orange: MOCCA MUSE equivalent mock observation, same bins as MUSE.

Fig. 8 the observational binary discovery fraction in relation to the projected distance to the centre of the globular cluster is shown. Since we do not have true distances to the cluster centre, any observed radial trend is expected to be somewhat weaker than the underlying trend with true radii. Nevertheless, we found a mild increase in the binary fraction towards the cluster centre. The slope of a simple linear fit is $(-0.055 \pm 0.003) \%$ per arcsec with a Pearson correlation coefficient of -0.28 . Despite the observational biases and the limited radial distance to the cluster centre this result agrees with the theoretical expectations. Compared to the mock simulation, for which the same projected radial bins have been applied, the result is qualitatively the same. We like to stress that the original MOCCA simulation has a clear radial trend in the binary frequency (using three dimensional radial bins).

\section{Determination of orbital parameters}

Constraining the orbital properties (six standard Keplerian parameters) of a binary system with very few or imprecise radial velocity measurements is difficult. Naively one could assume that in dense globular clusters only hard binaries (with high binding energy and typically short period) could survive and should circulise their orbit during their lifetime. However, not only due to dynamical interactions, a significant number of binaries are expected to be on eccentric orbits (Hut et al. 1992). Methods like the generalised Lomb-Scargle periodogram (GLS; Zechmeister $\&$ Kürster 2009) are good to find periods in unequally sampled data. GLS is good for finding periods in circular orbits, but tends to fail in eccentric configurations. The APOGEE team also faced the same challenges and created a tool named "THE JOKER" (Price-Whelan et al. 2017).

\subsection{THE JOKER}

THE JOKER is a custom Monte Carlo sampler for sparse or noisy radial velocity measurements of two-body systems and can produce posterior samples for orbital parameters even when the likelihood function is poorly behaved.

We follow the method of Price-Whelan et al. (2018) to find orbital solutions for our NGC 3201 stars. Our assumptions to use THE JOKER are accordingly: 

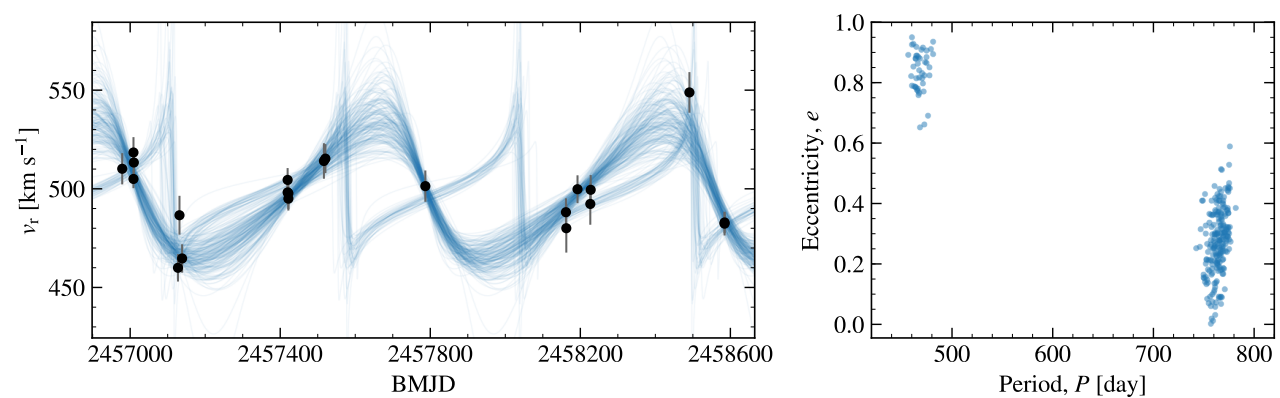

Fig. 9. Le ft: radial velocities (black data points) of the companion of the black hole candidate with ACS Id \#5132. The blue curves are the possible orbital solutions determined by THE JOKER for this star. Right: period-eccentricity plot of these samples.

- Two bodies: We assume the multiple star systems in NGC 3201 to consist of two stars or only two stars to be on the dominant timescale for the dynamics of the system (hierarchical multi-star system)

- SB1: The light of the binary components adds up in the MUSE spectrograph as a single source. We assume one of the two binary components does not contribute significantly to the spectrum (single-lined spectroscopic binary = SB1). In the case both components have similar magnitudes (double-lined spectroscopic binary $=\mathrm{SB} 2$ ) it is not possible for us to separate the components for typical radial velocity amplitudes, due to the low spectral resolution of MUSE. As mentioned in Sect. 4, the amplitude of SB2 systems depends on the flux ratio of the two binary components.

- Isolated Keplerian systems: The radial velocity variations are due to orbital motions and not caused by possible intrinsic variations (e.g. pulsations). Moreover, the cluster dynamics occurs on a longer timescale than the orbital motion in the binary system.

- Gaussian distributed uncertainties: We ensured all radial velocity uncertainties to be free from systematic effects, independent of each other and represent purely Gaussian noise (for details see Kamann et al. 2018, and Sect. 3.2).

We generated $2^{29}=536870912$ prior samples for the period range $0.3 \mathrm{~d}$ to $4096 \mathrm{~d}$. We requested 256 posterior samples for each star with a minimum of 5 observations and a minimum $50 \%$ probability to be a variable star. In our sample 515 met these conditions and could be processed with THE JOKER. If fewer than 200 posterior samples were found for one star, we used a dedicated Monte Carlo Markov chain (MCMC) run as described in Price-Whelan et al. (2017) to get 256 new samples. We analysed their posterior distributions and will focus on objects with unimodal or bimodal distributions in the following.

In Fig. 9 the result of this process for one example star (companion of a black hole candidate, more in Sect. 7.3) in our sample is presented. The 256 orbital solutions (in blue) represent the posterior likelihood distribution for this star and are in this case in principle two unique solutions with uncertainties. We define this solutions to be bimodal in orbital period. If in this example, only one solution (one cluster in the right panel of Fig. 9) would be present, we would define it as unimodal. In general, we have unimodal samples when the standard deviation of THE JOKER periods $P$ on logarithmic scale meets the criterion:

$\sigma_{\ln P}<0.5$.

To identify bimodal posterior samples we use a similar method as described in Price-Whelan et al. (2018): We use the $k$-means clustering with $k=2$ from the scikit-learn package (Pedregosa et al. 2011) to separate two clusters in the posterior samples in orbital period (like the two clusters in the right panel of Fig. 9). Each of the two clusters have to fulfil Eq. (7). We take the most probable (the one with more samples than the other) as the result for this bimodal posterior samples. Finally, we take the median and the standard deviation as uncertainty of all parameters from these results.

\subsection{Binary system properties}

The CMD in Fig. 10 is created using the newer HST UV globular cluster survey photometry of NGC 3201 (Nardiello et al. 2018; Piotto et al. 2015) which was matched to the photometry of the HST ACS globular cluster survey (Sarajedini et al. 2007; Anderson et al. 2008) we used as an input catalogue for the extraction of the spectra. The binary probability for each star we determined by our statistical method is colour-coded. As expected, many MS binaries positioned to the red of the MS are found by our method. Red giants brighter than the horizontal branch magnitude could not be found in binary systems in our sample. A good check for the statistical method are the chromospherically active binaries, which deviate in our CMD significantly from the MS (see annotation in CMD) and are very well confirmed by the method. More details about the other stellar types are presented in the following Sect. 7.

As described in Sect. 6.1 we analysed all stars with a $50 \%$ variability probability. We found 95 stars from which 78 stars have unimodal and 17 stars bimodal posterior samples in orbital period. That means the period of these stars is well constrained but does not mean all Keplerian parameters are constrained similarly well. We present the results of THE JOKER in Table A.2 with the star position, magnitude, a selection of the fitted parameters (period, eccentricity, amplitude, visible mass), and the derived invisible minimum (companion) mass. The comment column contains additional information to individual stars as explained in the table notes. For a selection of interesting stars of this table we show the best Keplerian fit in Fig. A.1 and a blind random set in Fig. A.2. These plots show in the upper panel the radial velocities $v_{r}$ of an individual star from our final sample phase folded with the period from the best fitting model. The lower panel contains the residuals after subtracting this model from the data. In addition to the identifier (ACS Id), position and magnitude from the ACS catalogue (Sarajedini et al. 2007), we note the period $P$, eccentricity $e$, invisible minimum mass $M_{2}$, reduced $\chi^{2}$ of the best fitting model, and the comment in every plot for convenience.

A3, page 10 of 20 
B. Giesers et al.: Binaries in NGC 3201

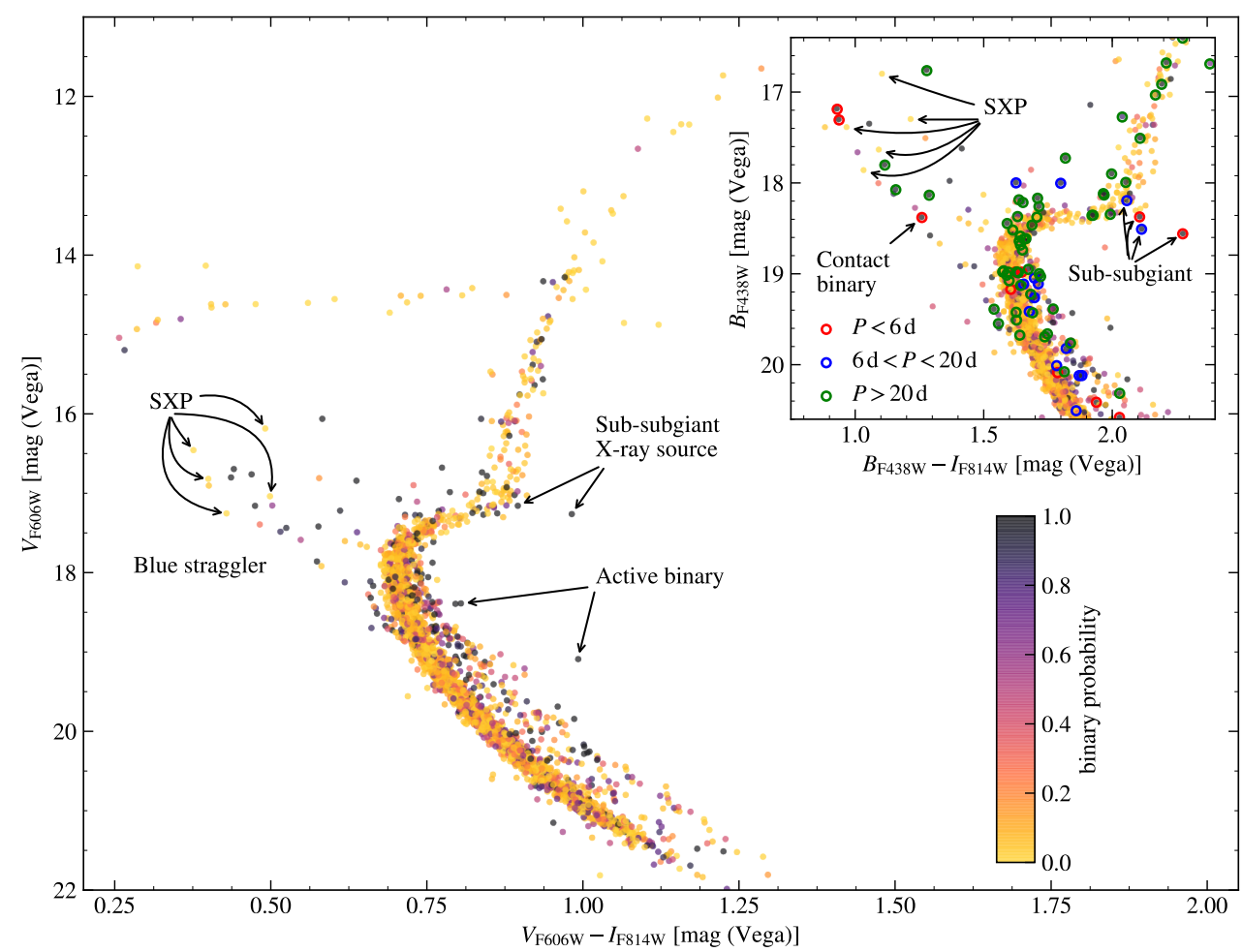

Fig. 10. Colour-magnitude diagram of the members of NGC 3201 created with the photometry taken from the HST UV globular cluster survey (Nardiello et al. 2018; Piotto et al. 2015). Colour-coded is the binary probability obtained by our statistical method. Large panel: full CMD of our sample using $V$ and $I$ equivalent filters. Small panel: detailed version of the MS turn-off CMD region using $B$ and $I$ equivalent filters. Additionally, the period $P$ range is indicated by coloured circles where THE JOKER was able to fit a well constrained Keplerian orbit.

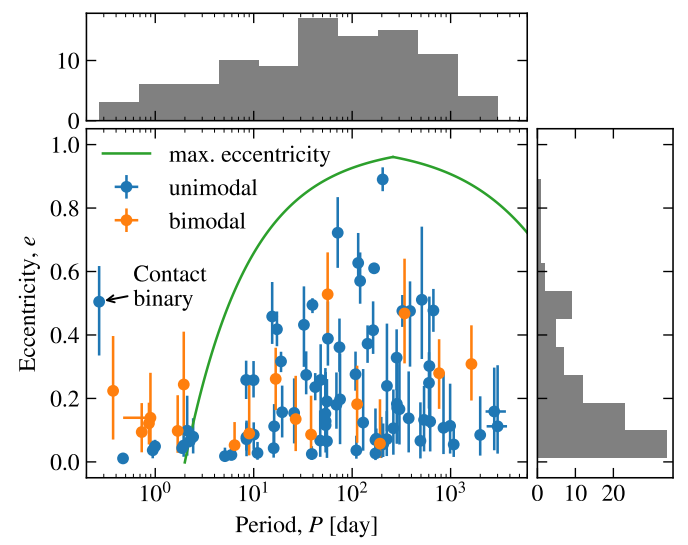

Fig. 11. Eccentricity-period plot of the well constrained binaries in NGC 3201. Binaries with unimodal (blue) and bimodal (orange) solutions in the posterior period sampling. The maximum eccentricity in green represents the theoretical limit (above a $2 \mathrm{~d}$ period) to which binary systems are stable (see Sect. 6.2).

For the first time Fig. 11 shows the eccentricity-period distribution of binaries in a globular cluster. We found binaries in a period range from $0.27 \mathrm{~d}$ to $3000 \mathrm{~d}$ with eccentricities from 0 to 0.9 . The eccentricity distribution is biased towards low eccentricities, because for these orbits fewer measurements are necessary to get a unique solution. In the figure we also show a maximum eccentricity $e_{\max }$ power law derived from a Maxwellian thermal eccentricity distribution

$e_{\max }(P)=1-\left(\frac{P}{2 \text { days }}\right)^{-2 / 3}$ for $P>2$ days

for a given period $P$ (Moe \& Di Stefano 2017). This represents the binary components having Roche lobe fill factors $\leq 70 \%$ at periastron. All binaries with $P<2 \mathrm{~d}$ should have circular orbits due to tidal forces. Additionally, we also take dynamical star interactions into account by using a limit on the orbital velocity at apoastron. The orbital velocity should always be higher than the central cluster dispersion of $5 \mathrm{~km} \mathrm{~s}^{-1}$ of NGC 3201 (Harris 1996, 2010 edition). This limit on eccentricity is dominant for periods $>260 \mathrm{~d}$. Except for the contact binary star (ACS Id: \#13108, see Table A.2) all stars behave accordingly. Note that when the eccentricity distribution of one star peaked at zero we still took the median like in all other distributions. Therefore the stars below $P<2$ are consistent within their uncertainty with $e=0$. Figure 11 also clearly shows that not all binaries in globular clusters have been circularised over the lifetime of a globular cluster. We, however, do not find high-eccentric long-period (e.g. $e>0.6$ and $P>300 \mathrm{~d}$ ) binaries within the eccentricity limit. 


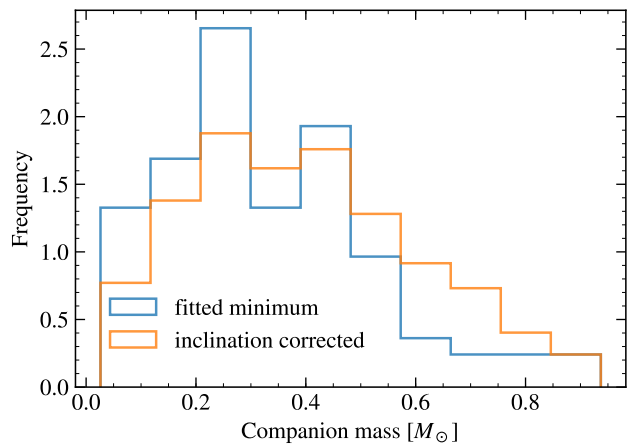

Fig. 12. Companion masses $\left(<1 M_{\odot}\right)$ of the well constrained binaries in NGC 3201 are shown. The blue histogram contains the fitted results (minimum masses) from THE JOKER, whereas the orange histogram contains the same results after applying a statistical correction for orbital inclination to obtain the actual companion mass distribution (see Sect. 6.2).

This could either be due to our observational bias or an effect which restricts the eccentricity even further.

Figure 12 shows the minimum companion masses of the well constrained binaries (blue histogram). In orange all masses are resampled 1000 times from their fitted mass divided by $\sin i$ to obtain the actual companion mass distribution. The inclination $i$ is taken from a sinusoidal detection probability distribution between 0 to $\pi / 2$ with a cutoff mass of $0.8 M_{\odot}$ (roughly maximum normal stellar mass in NGC 3201). This results in a linear anti-correlation between the companion mass and the frequency of binaries having this companion mass. As explained in Sect. 4 we have a bias towards binaries with both components having different luminosities. Milone et al. (2012) found a flat mass ratio distribution throughout all clusters above a mass ratio $>0.5$. The pairing fraction of our binaries is beyond the scope of this paper, since we would need to correct for the luminosity ratio effect and the selection function of THE JOKER, which is currently unknown in our case.

Noteworthy is that all red giant binaries in our sample with well constrained orbits have periods larger than $100 \mathrm{~d}$. This corresponds to a minimum semi-major axis of $\gtrsim 0.5 \mathrm{AU}$ and is consistent with not having Roche-lobe overflow in the binary system.

\section{Peculiar objects in NGC 3201}

\subsection{Blue straggler stars and SX Phoenicis-type stars}

Our sample of NGC 3201 contains 40 blue stragglers with sufficient observations and signal to noise. From these stars, 23 are most likely in binary systems while 17 are not (including the SX Phoenicis-type stars assumed to be single stars). This observational binary fraction of $(57.5 \pm 7.9) \%$ is much higher than the one of all cluster stars or even the Milky Way field stars (see Sect. 1). Our ratio of binaries to single blue straggler stars $R_{\mathrm{B} / \mathrm{S}}=1.35$ in the core of NGC 3201 is significantly higher than the prediction by Hypki \& Giersz (2017) of $R_{\mathrm{B} / \mathrm{S}} \sim 0.4$, but could also be explained by an overabundance of the more massive blue straggler binaries (compared to single blue stragglers) due to mass segregation. Nevertheless, this high binary fraction strongly suggests that most of the blue stragglers in our sample were formed within a binary system. As in Sect. 2 explained, a cluster member needs additional mass to become a blue straggler. Two formation scenarios are consistent with this high binary fraction: (1) mass transfer within a triple star system (e.g. Antonini et al. 2016), (2) stellar mergers induced by stellar interactions of binary systems with other binaries or single stars (e.g. Leonard 1989; Fregeau et al. 2004).

From these 23 blue straggler binaries THE JOKER could find 11 highly constrained solutions, three are extremely hard binaries $(<1 \mathrm{~d})$ with a minimum companion mass ranging from $0.11 M_{\odot}$ to $0.24 M_{\odot}$ and six relatively wide $(>100 \mathrm{~d})$ with minimum companion masses ranging from $0.35 M_{\odot}$ to $0.67 M_{\odot}$. The remaining 2 blue stragglers are in between the period and companion mass range (see stars in Table A. 2 with comment "BSS" for more details). Figure 10 shows the blue stragglers and their period range in a CMD. We also identified five blue straggler stars as known SX Phoenicis-type variable stars in the Catalogue of Variable Stars in Galactic Globular Clusters (Clement et al. 2001; Clement 2017) and one of them as a SX Phoenicis candidate in Arellano Ferro et al. (2014). We also see their pulsations (similar to the RR Lyrae-type stars) as a radial velocity signal up to amplitudes of $18 \mathrm{~km} \mathrm{~s}^{-1}$. We cannot distinguish between the pulsation signal and a possible signal induced by a binary companion, thus we consider these stars to be single stars and the velocity variability solely explained by radial pulsations.

Mass transfer in a relatively wide blue straggler binary system is almost impossible. Thus, only the three hard binaries probably result from this process. It is obvious that mass transfer is taking place in the contact binary system with ACS Id \#13108. On the other hand, head-on collisions of single stars would result in single blue stragglers and are extremely unlikely in a low-density cluster such as NGC 3201. Therefore, it is more likely that a high percentage of the blue stragglers in our sample result from coalescence in systems with $\geq 3$ stars. The complex behaviour of such systems favours configurations that result in the coalescence of two of its members (e.g. Leonard 1989; Fregeau et al. 2004; Antonini et al. 2016). If more than two stars in the formation of blue stragglers are involved, this could also mean that hard blue straggler binary systems could have a third outer component inducing an additional, much weaker signal. We could not identify such a signal in our data. For example, the star with ACS Id \#12746 (see radial velocity signal in Fig. A.1) is the most central hard blue straggler binary in our sample with 63 observations, but its radial velocity curve does not contain any significant additional signal.

The projected spin velocities $(V \sin i)$ of blue stragglers have been suggested as a possibility to infer their ages and origins (e.g. Leiner et al. 2018). $V \sin i$ measurements of blue stragglers in NGC 3201 have been performed by Simunovic \& Puzia (2014) and cross-matching their sample with our data results in a subset of five blue stragglers with spin velocities ranging from $(34.6 \pm 1.6) \mathrm{km} \mathrm{s}^{-1}$ to $(135.2 \pm 3.5) \mathrm{km} \mathrm{s}^{-1}$. We note that all of them are binary systems. However, no clear picture emerges regarding a possible relation between spin velocity and orbital properties.

As introduced, SX Phoenicis-type stars are most likely formed in binary evolution. That is why it is possible for them to have a companion, but the companions radial velocity signal would be hidden in the pulsation signal.

\subsection{Sub-subgiant stars}

We found four SSG stars ${ }^{4}$ within our MUSE FoV (see position in Fig. 10). Assuming the radial velocity changes are only

4 The four SSGs in our sample could also be defined as red stragglers, since they are at the boundary of both definitions in Geller et al. (2017a). 
Keplerian (and not by pulsations), THE JOKER could determine unique Keplerian solutions for all of them (see stars in Table A.2 with comment "SSG").

Fortunately the SSG with ACS Id \#14749 (see also plot in Fig. A.1) is a known detached eclipsing binary with a period of $10.0037 \mathrm{~d}$ (Kaluzny et al. 2016) and we found the same period $(10.006 \pm 0.002) \mathrm{d}$ with our method. But this also means the system is observed edge-on and our companion mass is its true mass. The visible star shows a radial velocity amplitude of $(42.9 \pm 1.5) \mathrm{km} \mathrm{s}^{-1}$ with a very low eccentricity of $(0.09 \pm 0.07)$, and a companion mass of $(0.53 \pm 0.04) M_{\odot}$ for an assumed primary mass of $(0.82+0.05) M_{\odot}$. That makes a system mass of $1.35 M_{\odot}$ and considering mass transfer, one possible pathway could be towards the blue straggler region (Leiner et al. 2017). Compared to our best fitting model spectrum (and to a well fitted $\mathrm{H} \beta$ line) all spectra of this star show a partially filled-in $\mathrm{H} \alpha$ absorption line (the absorption is not as deep as expected, see Fig. A.3 as an example). This could mean that mass transfer is already underway. In this case this could be used to infer an accretion rate, but is beyond the scope of this paper.

The two short period ( $<6 \mathrm{~d}, \# 22692$, \#13438) SSGs show X-ray emission according to the November 2017 pre-release of the Chandra Source Catalog Release 2.0 (Evans et al. 2010). The SSG (\#22692) with the $5.1 \mathrm{~d}$ period actually has significant varying $\mathrm{H} \alpha$ emission lines in most of our spectra. One spectrum of this star with $\mathrm{H} \alpha$ in emission is presented in Fig. A.4. The maximum emission line is twice as strong as the minimum line compared to the continuum. Again, it would be interesting to calculate the accretion rate for this case, but is beyond the scope of this paper. The SSG (\#13438) with the $5.9 \mathrm{~d}$ period has partially filled-in $\mathrm{H} \alpha$ absorption lines in all spectra like the eclipsing SSG described before. One spectrum of this star with the filled-in $\mathrm{H} \alpha$ line is shown in Fig. A.3. Both short period SSGs have a similar semi-amplitude of $\sim 37 \mathrm{~km} \mathrm{~s}^{-1}$, no eccentricity and a minimum companion mass of $(0.35 \pm 0.03) M_{\odot}$.

The SSG (\#11405) has with $17.2 \mathrm{~d}$ the longest period, unlike the other stars a relatively high eccentricity of 0.4 and a low minimum companion mass of $0.15 M_{\odot}$.

The detection of a dozen more SSGs and RSs in our MUSE globular cluster sample will be published in the emission line catalogue of Göttgens et al. (2019b).

\subsection{Black hole candidates}

Here we report our new measurements for the stellar-mass black hole $(\mathrm{BH})$ candidate in NGC 3201 previously published in Giesers et al. (2018). The additional observations perfectly match the known orbital model and confirm the $\mathrm{BH}$ minimum mass to be $(4.53 \pm 0.21) M_{\odot}$. The deviation to the previously minimum mass of $(4.36 \pm 0.41) M_{\odot}$ is within the uncertainties. Furthermore, the observations fill in the apoapsis which was missed by previous observations (see \#12560 in Fig. A.1) and exclude other possible orbital solutions. We would like to emphasise that this star has been analysed with THE JOKER and MCMC like all other stars, without the effort invested in it as in Giesers et al. (2018).

In our full sample of NGC 3201 we detected two additional BH candidates with solutions from the fit of THE JOKER. Table A. 2 shows the resulting properties of these binary systems with ACS Id \#21859 and \#5139. Both systems are faint with extracted spectra of $S / N \leq 10$. Remarkable is the system (\#21859) with a unique solution and a semi-amplitude of about $300 \mathrm{~km} \mathrm{~s}^{-1}$, we cannot explain the radial velocity curve shown in Fig. A.1 with other explanations than an unseen companion with a minimum mass of $(7.68 \pm 0.50) M_{\odot}$. The other system (\#5132) has two solutions with the more probable ( $82 \%$ probability) minimum companion mass of $(4.40 \pm 2.82) M_{\odot}$ and the less probable of $1.10 \pm 0.20 M_{\odot}$. This candidate is not well constrained and needs more observations to be confirmed as a $\mathrm{BH}$. To our knowledge, both sources do not have an X-ray or radio counterpart. This is remarkable in the case of \#21859, as the presence of a partially filled-in $\mathrm{H} \alpha$ line in the MUSE spectra suggests that accretion is present. We show a combination of all spectra shifted to rest-frame in Fig. A.5.

In light of the recent results based on Monte Carlo simulations, it appears feasible that NGC 3201 hosts several BHs in binaries with MS stars. Both Kremer et al. (2018) and Askar et al. (2018a) predict the presence of at least $\sim 100 \mathrm{BHs}$ in the cluster, with tens of them in binaries with other BHs or bright companions More specifically, in the best model of Kremer et al. (2018), four $\mathrm{BHs}$ have MS-star companions. Interestingly, all of those systems show eccentricities $\gtrsim 0.6$, add odds with the new candidates that we discovered (cf. Table A.2). On the other hand, the semi-major axes of our candidates of $0.067 \mathrm{AU}$ and $2.804 \mathrm{AU}$ are within the range of semi-major-axis values that Kremer et al. (2018) found in their best model of NGC 3201 (see their Fig. 3).

Our MOCCA snapshot at $12 \mathrm{Gyr}$ contains $43 \mathrm{BHs}$ with 33 single $\mathrm{BHs}, 2 \mathrm{BH}-\mathrm{BH}$ binaries, and $6 \mathrm{BH}-\mathrm{star}$ binaries. The median mass of all BHs is $16.8 M_{\odot}$ within a range of $3.7 M_{\odot}$ to $78 M_{\odot}$. Two $\mathrm{BH}$-star binaries and 5 single $\mathrm{BHs}$ are in the probable mass region of the $\mathrm{BH}$ candidates we discussed here. The total number of $\mathrm{BHs}$ is lower than the previous predictions, but the number of BHs with detectable companions is consistent with our observations throughout all models.

\section{Conclusions and outlook}

We elaborated the first binary study from our MUSE survey of 27 Galactic globular clusters in the core of NGC 3201 and showed the variety of results our blind multi-epoch spectroscopic observations reveal. Since modelling a globular cluster contains many imponderables, we developed a statistical method - applicable to any time-variant measurements with inhomogeneous sampling and uncertainties (not only radial velocities) - which can distinguish between a constant and varying signal (like in single and binary stars). We determined an observational binary frequency of $(20.08 \pm 0.22) \%$ using this statistical method. Based on a comparison with an advanced MOCCA simulation of NGC 3201, we calculated a total binary fraction of $(6.75 \pm 0.72) \%$ for all stars in the whole cluster. We confirmed the trend of an increasing binary fraction towards the cluster centre due to mass segregation. We found a significantly higher binary fraction $(57.5 \pm 7.9) \%$ of blue straggler stars compared to the cluster, indicating that the formation of blue stragglers in the core of NGC 3201 is related to binary evolution. For the first time, we presented well constrained Keplerian orbit solutions for a significant amount of stars (95) using the Monte Carlo tool THE JOKER. Eleven of these are blue straggler stars very likely in binary systems and shed light on the properties of these systems. We conclude that both the mass transfer formation scenario and the collisional formation scenario of blue stragglers are present in our data. Collision means in this case the coalescence of two stars during a binary-binary or binary-single encounter. We found four SSG stars by connecting our MUSE spectroscopy with HST photometry and X-ray observations. Fortunately, we got definite Keplerian solutions for all of them and have insights into their properties for the first time in a globular cluster. Finally, we presented three stellar-mass BH candidates, from which one is already published (Giesers et al. 2018) and one with a minimum mass of 
$(7.68 \pm 0.50) M_{\odot}$ is clearly above a single and even binary neutron star companion mass limit. In total, these BH candidates in binary systems with MS stars would strongly support the hypothesis that NGC 3201 has an extensive BH population of up to hundred more BHs (Kremer et al. 2018; Askar et al. 2018a). This cluster, and maybe other globular clusters as well, could be a significant source of gravitational waves.

In a following paper we will present binary fractions in the context of multiple stellar populations within NGC 3201. We will continue to observe $\omega$ Cen and 47 Tuc to have a comparable amount of epochs and will end up with a magnitude more stars per cluster compared to NGC 3201. Finally, we will publish the binary fractions of all clusters in our survey and try to find correlations with cluster parameters.

Acknowledgements. We thank Arash Bahramian, Nate Bastian, Robert Mathieu, Wolfram Kollatschny, Stan Lai, and Mark Gieles for helpful discussions. BG, SD, SK and PMW acknowledge support from the German Ministry for Education and Science (BMBF Verbundforschung) through grants 05A14MGA, 05A17MGA, 05A14BAC, and 05A17BAA. AA is supported by the Carl Tryggers Foundation for Scientific Research through the grant CTS 17:113. SK gratefully acknowledges funding from a European Research Council consolidator grant (ERC-CoG-646928- Multi-Pop). JB acknowledges support by FCT/MCTES through national funds by grant UID/FIS/04434/2019 and through Investigador FCT Contract No. IF/01654/2014/CP1215/CT0003. This research is supported by the German Research Foundation (DFG) with grants DR 281/351 and KA 4537/2-1. Based on observations made with ESO Telescopes at the La Silla Paranal Observatory under programme IDs 094.D-0142, 095.D-0629, 096.D-0175, 097.D-0295, 098 D-0148, 0100.D-0161,0101.D-0268, 0102.D0270, and 0103.D-0204. Bad on observation made wh the NASA/ESA Hubble Space Telescope, obtained fom the dat archive at he Space Telescope Scible Space Telescope, obtained from the data archive at the Space Telescope Science Institute. STScI is operated by the Association of Universities for Research in Astronomy, Inc. under NASA contract NAS 5-26555. Supporting data for this article is available at: http://musegc.uni-goettingen.de.

\section{References}

Anderson, J., Sarajedini, A., Bedin, L. R., et al. 2008, AJ, 135, 2055 Antonini, F., Chatterjee, S., Rodriguez, C. L., et al. 2016, ApJ, 816, 65 Arca Sedda, M., Askar, A., \& Giersz, M. 2018, MNRAS, 479, 4652

Arellano Ferro, A., Ahumada, J. A., Calderón, J. H., \& Kains, N. 2014, Rev. Mex. Astron. Astrofis., 50, 307

Askar, A., Szkudlarek, M., Gondek-Rosińska, D., Giersz, M., \& Bulik, T. 2017, MNRAS, 464, L36

Askar, A., Arca Sedda, M., \& Giersz, M. 2018a, MNRAS, 478, 1844

Askar, A., Giersz, M., Pych, W., \& Dalessandro, E. 2018b, MNRAS, 475, 4170

Askar, A., Askar, A., Pasquato, M., \& Giersz, M. 2019, MNRAS, 485, 5345

Baldwin, A. T., Watkins, L. L., van der Marel, R. P., et al. 2016, ApJ, 827, 12

Baumgardt, H. 2017, MNRAS, 464, 2174

Baumgardt, H., \& Hilker, M. 2018, MNRAS, 478, 1520

Belczynski, K., Kalogera, V., \& Bulik, T. 2002, ApJ, 572, 407

Belloni, D., Askar, A., Giersz, M., Kroupa, P., \& Rocha-Pinto, H. J. 2017 MNRAS, 471, 2812

Belloni, D., Giersz, M., Rivera Sandoval, L. E., Askar, A., \& Ciecielåg, P. 2019, MNRAS, 483, 315

Bressan, A., Marigo, P., Girardi, L., et al. 2012, MNRAS, 427, 127

Chatterjee, S., Rodriguez, C. L., \& Rasio, F. A. 2017, ApJ, 834, 68

Cheng, Z., Li, Z., Xu, X., \& Li, X. 2018, ApJ, 858, 33

Cheng, Z., Li, Z., Li, X., Xu, X., \& Fang, T. 2019, ApJ, 876, 59

Clement, C. M. 2017, VizieR Online Data Catalog: V/150

Clement, C. M., Muzzin, A., Dufton, Q., et al. 2001, AJ, 122, 2587

Cohen, R. E., \& Sarajedini, A. 2012, MNRAS, 419, 342

Dotter, A., Sarajedini, A., Anderson, J., et al. 2010, ApJ, 708, 698

Duchêne, G., \& Kraus, A. 2013, ARA\&A, 51, 269

Duquennoy, A. \& Mayor, M. 1991, A\& A, 248, 485

Evans, I. N., Primini, F. A., Glotfelty, K. J., et al. 2010, ApJS, 189, 37

Ferraro, F. R., Paltrinieri, B., Fusi Pecci, F, et al. 1997, A\&A, 324, 915

Ferraro, F. R., Paltrinieri, B., Fusi Pecci, F., et al. 1997, A\&A, 324, 915

Fregeau, J. M., Cheung, P., Portegies Zwart, S. F., \& Rasio, F. A. 2004, MNRAS, 352,1

Fregeau, J. M., Ivanova, N., \& Rasio, F. A. 2009, ApJ, 707, 1533
Geller, A. M. \& Mathieu, R. D. 2011, Nature, 478, 356

Geller, A. M., Leiner, E. M., Bellini, A., et al. 2017a, ApJ, 840, 66

Geller, A. M., Leiner, E. M., Chatterjee, S., et al. 2017b, ApJ, 842, 1 Giersz, M. 1998, MNRAS, 298, 1239

Giersz, M., Heggie, D. C., Hurley, J. R., \& Hypki, A. 2013, MNRAS, 431, 2184

Giesers, B., Dreizler, S., Husser, T.-O., et al. 2018, MNRAS, 475, L15 Goodman, J., \& Hut, P. 1989, Nature, 339, 40

Göttgens, F., Weilbacher, P. M., Roth, M. M., et al. 2019a, A\&A, 626, A69

Göttgens, F., Husser, T. O., Kamann, S., et al. 2019b, A\&A, 631, A118

Harris, W. E. 1996, AJ, 112, 1487

Hénon, M. H. 1971, Ap\&SS, 14, 151

Hobbs, G., Lorimer, D. R., Lyne, A. G., \& Kramer, M. 2005, MNRAS, 360, 974

Hong, J., Vesperini, E., Askar, A., et al. 2018, MNRAS, 480, 5645 Hurley, J. R., Pols, O. R., \& Tout, C. A. 2000, MNRAS, 315, 543 Hurley, J. R., Tout, C. A., \& Pols, O. R. 2002, MNRAS, 329, 897 Hurley, J. R., Aarseth, S. J., \& Shara, M. M. 2007, ApJ, 665, 707 Husser, T.-O., Wende-von Berg, S., Dreizler, S., et al. 2013, A\&A, 553, A6

Husser, T.-O., Kamann, S., Dreizler, S., et al. 2016, A\&A, 588, A148 Husser, T. O., Latour, M., Brinchmann, J., et al. 2019, A\&A, submitted Hut, P., McMillan, S., Goodman, J., et al., 1992, PASP, 104, 981

Hypki, A., \& Giersz, M. 2013, MNRAS, 429, 1221

Hypki, A., \& Giersz, M. 2017, MNRAS, 466, 320

Ivanova, N., Belczynski, K., Fregeau, J. M., \& Rasio, F. A. 2005, MNRAS, 358 572

Joshi, K. J., Rasio, F. A., \& Portegies Zwart, S. 2000, ApJ, 540, 969 Kaluzny, J., Rozyczka, M., Thompson, I. B., et al. 2016, Acta Astron., 66, 31 Kamann, S., Wisotzki, L., \& Roth, M. M. 2013, A\&A, 549, A71 Kamann, S., Husser, T.-O., Dreizler, S., et al. 2018, MNRAS, 473, 5591 Kim, C., McNamara, D. H., \& Christensen, C. G. 1993, AJ, 106, 2493 King, I. R. 1966, AJ, 71, 64

Kremer, K., Ye, C. S., Chatterjee, S., Rodriguez, C. L., \& Rasio, F. A. 2018, ApJ, 855, L15

Kroupa, P. 2001, MNRAS, 322, 231

Latour, M., Husser, T. O., Giesers, B., et al. 2019, A\&A, 631, A14 Leigh, N. W. C., Giersz, M., Marks, M., et al. 2015, MNRAS, 446, 226 Leiner, E., Mathieu, R. D., \& Geller, A. M. 2017, ApJ, 840, 67 Leiner, E., Mathieu, R. D., Gosnell, N. M., \& Sills, A. 2018, ApJ, 869, L29 Leonard, P. J. T. 1989, AJ, 98, 217

McLaughlin, D. E., \& van der Marel, R. P. 2005, ApJS, 161, 304

Milone, A. P., Piotto, G., Bedin, L. R., et al. 2012, A\&A, 540, A16 Moe, M., \& Di Stefano, R. 2017, ApJS, 230, 15

Morscher, M., Pattabiraman, B., Rodriguez, C., Rasio, F. A., \& Umbreit, S. 2015, ApJ, 800,9

Nardiello, D., Libralato, M., Piotto, G., et al. 2018, MNRAS, 481, 3382

Pattabiraman, B., Umbreit, S., Liao, W.-K., et al. 2013, ApJS, 204, 15

Pedregosa, F., Varoquaux, G., Gramfort, A., et al. 2011, J. Mach. Learn. Res., 12,2825

Piotto, G., Milone, A. P., Bedin, L. R., et al. 2015, AJ, 149, 91

Price-Whelan, A. M., Hogg, D. W., Foreman-Mackey, D., \& Rix, H.-W. 2017, ApJ, 837, 20

Price-Whelan, A. M., Hogg, D. W., Rix, H.-W., et al. 2018, AJ, 156, 18

Rivera Sandoval, L. E., van den Berg, M., Heinke, C. O., et al. 2018, MNRAS, 475,4841

Rodriguez, C. L., Chatterjee, S., \& Rasio, F. A. 2016, Phys. Rev. D, 93, 084029 Sarajedini, A., Bedin, L. R., Chaboyer, B., et al. 2007, AJ, 133, 1658 Simunovic, M., \& Puzia, T. H. 2014, ApJ, 782, 49

Sollima, A., Beccari, G., Ferraro, F. R., Fusi Pecci, F., \& Sarajedini, A. 2007, MNRAS, 380, 781

Sollima, A., Lanzoni, B., Beccari, G., Ferraro, F. R., \& Fusi Pecci, F. 2008, A\&A, 481,701

Sommariva, V., Piotto, G., Rejkuba, M., et al. 2009, A\&A, 493, 947

Strader, J., Chomiuk, L., Maccarone, T. J., Miller-Jones, J. C. A., \& Seth, A. C. 2012, Nature, 490, 71

Tonry, J., \& Davis, M. 1979, AJ, 84, 151

Tremou, E., Strader, J., Chomiuk, L., et al. 2018, ApJ, 862, 16

Wang, L., Spurzem, R., Aarseth, S., et al. 2016, MNRAS, 458, 1450

Weilbacher, P. M., Streicher, O., Urrutia, T., et al. 2012, in Software and Cyberinfrastructure for Astronomy II, Proc. SPIE, 8451, 84510B

Weilbacher, P. M., Streicher, O., Urrutia, T., et al. 2014, in Astronomical Data Analysis Software and Systems XXIII, eds. N. Manset, P. Forshay, et al., ASP Analysis Software

Wendt, M., Husser, T.-O., Kamann, S., et al. 2017, A\&A, 607, A133

Zechmeister, M., \& Kürster, M. 2009, A\&A, 496, 577

Zocchi, A., Gieles, M., \& Hénault-Brunet, V. 2019, MNRAS, 482, 4713 


\section{Appendix A: Additional tables and figures}
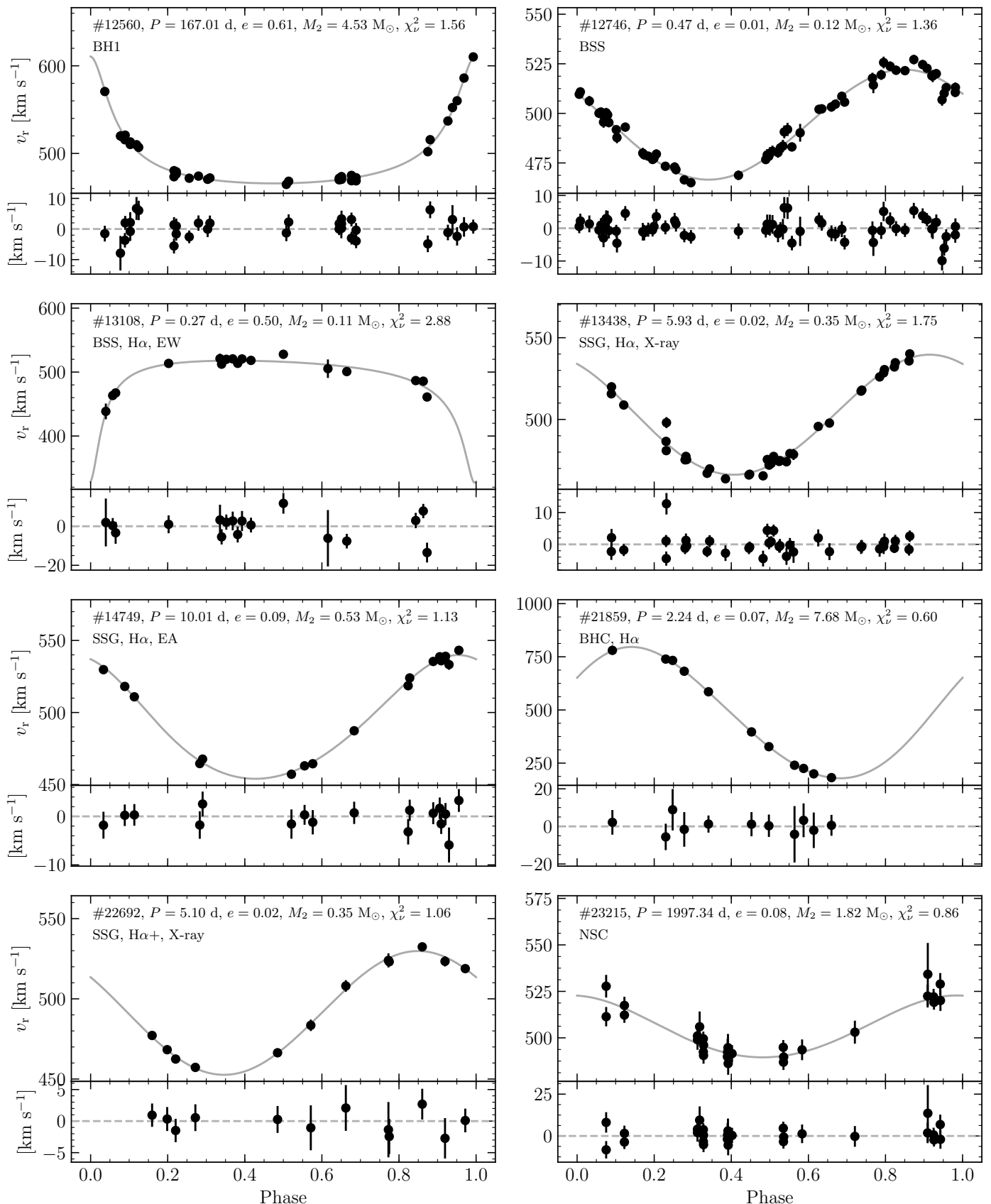

Fig. A.1. Selection of interesting results from THE JOKER sorted by ACS Id. The upper panel of every plot shows the radial velocities of the star from our final sample phase folded with the period from the best-fitting model. (Sometimes the uncertainties appear smaller as the data points.) The best-fitting model is plotted with the continues line. The lower panel contains the residuals after subtracting this model from the data. We note the source number, period $P$, eccentricity $e$, invisible mass $M_{2}$ and reduced $\chi^{2}$ of the best fitting model in every plot. See Table 1 for more information to individual stars. 


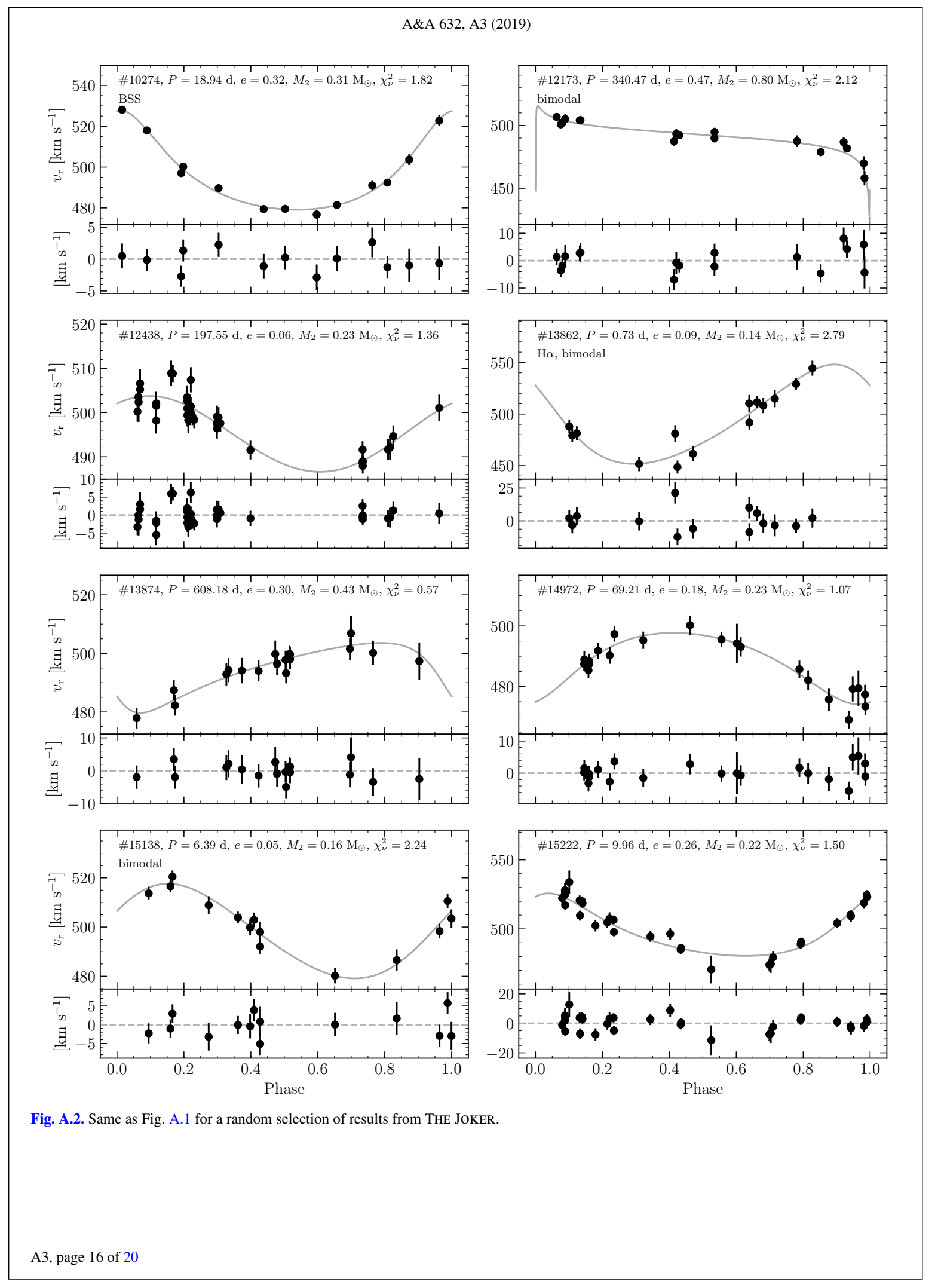


B. Giesers et al.: Binaries in NGC 3201

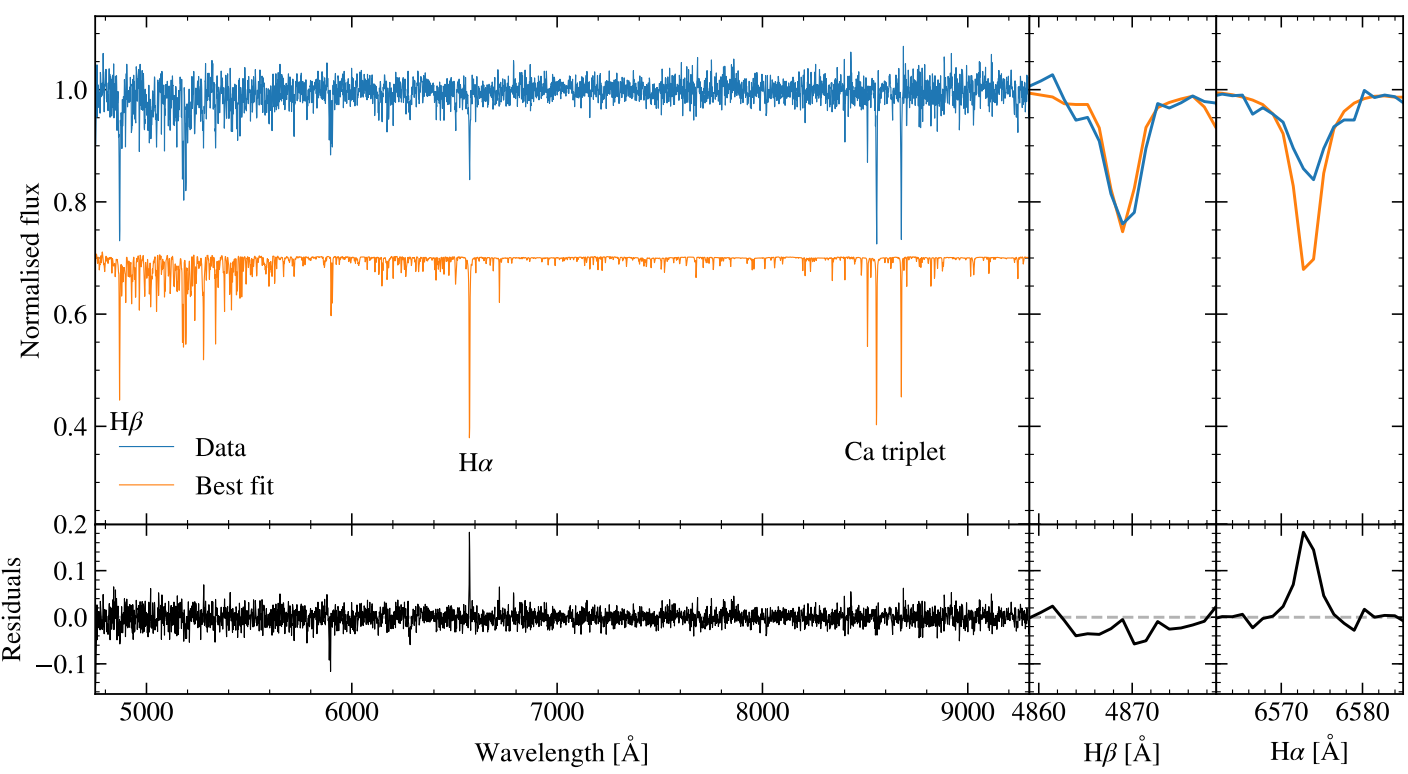

Fig. A.3. Spectrum of the sub-subgiant with ACS Id \#13438. Top panel: one normalised observed spectrum in blue and the best-fitting PHOENIX spectrum offset by 0.3 in orange. Bottom panel: residuals after subtracting the best fit from the data. Right panels: zoom at the $\mathrm{H} \beta$ and $\mathrm{H} \alpha$ line with a filled-in observed $\mathrm{H} \alpha$ line in contrast to the best fit (not offset).

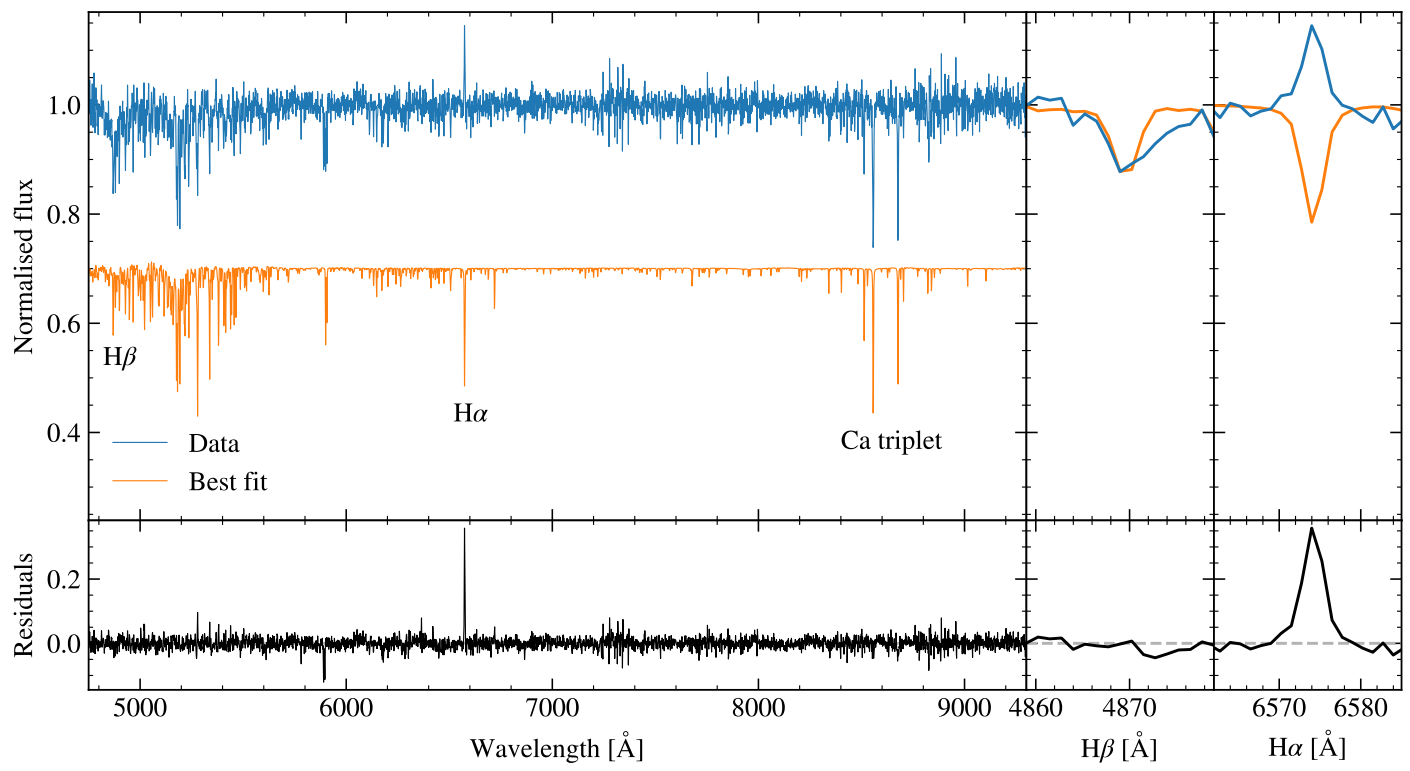

Fig. A.4. Same as Fig. A.3 for the sub-subgiant with ACS Id \#22692. Note that in contrast to star \#13438 (cf. Fig. A.3), this star shows an observed $\mathrm{H} \alpha$ emission line. 


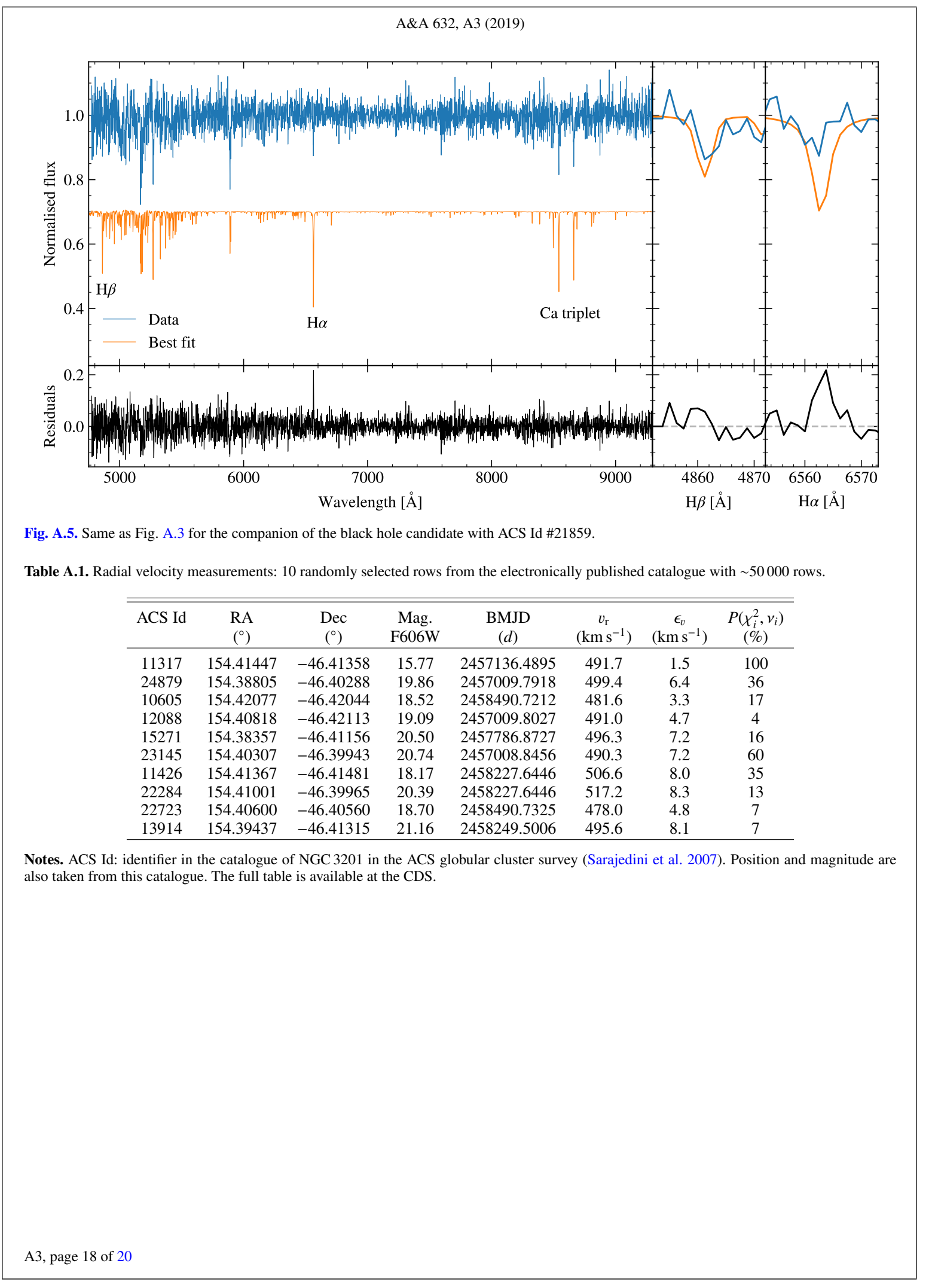


A stellar census in globular clusters with MUSE: Binaries in NGC 3201

B. Giesers et al.: Binaries in NGC 3201

Table A.2. Binary system properties.

\begin{tabular}{|c|c|c|c|c|c|c|c|c|c|c|}
\hline ACS Id & $\begin{array}{l}\text { RA } \\
\left({ }^{\circ}\right)\end{array}$ & $\begin{array}{l}\text { Dec } \\
\left({ }^{\circ}\right)\end{array}$ & $\begin{array}{l}\text { Mag. } \\
\text { F606W }\end{array}$ & $\begin{array}{r}\text { Peri } \\
\text { (d }\end{array}$ & & Eccentricity & $\begin{array}{c}\text { Amplitude } \\
\left(\mathrm{km} \mathrm{s}^{-1}\right)\end{array}$ & $\begin{array}{c}\text { Vis. mass } \\
\left(M_{\odot}\right)\end{array}$ & $\begin{array}{l}\text { Invis. min. mass } \\
\quad\left(M_{\odot} \sin i\right)\end{array}$ & Comment \\
\hline 13108 & 154.40005 & -46.42014 & 17.68 & 0.269 & \pm 0.007 & $0.50 \pm 0.21$ & $33.4 \pm 14.3$ & $1.20 \pm 0.05$ & $0.11 \pm 0.05$ & BSS, $\mathrm{H} \alpha$, EW \\
\hline 25210 & 154.38502 & -46.40803 & 18.05 & 0.3731 & \pm 0.0001 & $0.22 \pm 0.24$ & $9.2 \pm 2.3$ & $0.80 \pm 0.05$ & $0.03 \pm 0.01$ & $\mathrm{H} \alpha$, bimodal \\
\hline 12746 & 154.40330 & -46.41368 & 16.72 & 0.4706 & \pm 0.0001 & $0.01 \pm 0.02$ & $28.0 \pm 0.8$ & $1.20 \pm 0.05$ & $0.12 \pm 0.01$ & BSS, $V \sin i$ \\
\hline 13862 & 154.39460 & -46.42221 & 20.21 & 0.7287 & \pm 0.0001 & $0.09 \pm 0.12$ & $41.9 \pm 6.4$ & $0.60 \pm 0.05$ & $0.14 \pm 0.03$ & $\mathrm{H} \alpha$, bimodal \\
\hline 22697 & 154.40615 & -46.41101 & 19.41 & 0.8631 & \pm 0.0001 & $0.12 \pm 0.10$ & $30.7 \pm 2.4$ & $0.69 \pm 0.05$ & $0.12 \pm 0.02$ & bimodal \\
\hline 12581 & 154.40447 & -46.41137 & 19.09 & 0.89 & \pm 0.43 & $0.14 \pm 0.18$ & $27.1 \pm 3.3$ & $0.72 \pm 0.05$ & $0.10 \pm 0.02$ & bimodal \\
\hline 11222 & 154.41516 & -46.42245 & 16.82 & 0.9430 & \pm 0.0001 & $0.04 \pm 0.04$ & $37.4 \pm 1.8$ & $1.20 \pm 0.05$ & $0.22 \pm 0.02$ & BSS, $V \sin i$ \\
\hline 13362 & 154.39838 & -46.41245 & 20.86 & 0.9954 & \pm 0.0001 & $0.05 \pm 0.03$ & $124.8 \pm 4.0$ & $0.57 \pm 0.05$ & $0.68 \pm 0.06$ & $\mathrm{H} \alpha$ \\
\hline 12229 & 154.40736 & -46.41683 & 19.83 & 1.6900 & \pm 0.0003 & $0.10 \pm 0.14$ & $15.0 \pm 2.9$ & $0.66 \pm 0.05$ & $0.07 \pm 0.02$ & bimodal \\
\hline 10293 & 154.42364 & -46.41268 & 18.05 & 1.8760 & \pm 0.0001 & $0.04 \pm 0.03$ & $66.3 \pm 1.7$ & $0.80 \pm 0.05$ & $0.44 \pm 0.03$ & \\
\hline 13968 & 154.39393 & -46.41370 & 19.29 & 1.9429 & \pm 0.0001 & $0.05 \pm 0.06$ & $80.9 \pm 7.6$ & $0.71 \pm 0.05$ & $0.55 \pm 0.08$ & $\mathrm{H} \alpha$ \\
\hline 22818 & 154.40547 & -46.39993 & 18.25 & 1.9477 & \pm 0.0002 & $0.24 \pm 0.24$ & $14.7 \pm 5.7$ & $0.79 \pm 0.05$ & $0.08 \pm 0.03$ & bimodal \\
\hline 11802 & 154.41090 & -46.41574 & 19.70 & 2.11 & \pm 0.20 & $0.10 \pm 0.14$ & $20.0 \pm 2.7$ & $0.64 \pm 0.05$ & $0.10 \pm 0.02$ & $\mathrm{H} \alpha$ \\
\hline 21859 & 154.41347 & -46.40691 & 20.37 & 2.2422 & \pm 0.0001 & $0.07 \pm 0.04$ & $305.5 \pm 7.7$ & $0.61 \pm 0.05$ & $7.68 \pm 0.50$ & $\mathrm{BHC}$, \\
\hline 12836 & 154.40215 & -46.42134 & 20.13 & 2.4262 & \pm 0.0002 & $0.08 \pm 0.08$ & $44.3 \pm 2.5$ & $0.63 \pm 0.05$ & $0.26 \pm 0.03$ & $\mathrm{H} \alpha$ \\
\hline 22692 & 154.40662 & -46.39785 & 17.25 & 5.1038 & \pm 0.0004 & $0.02 \pm 0.03$ & $38.6 \pm 1.6$ & $0.82 \pm 0.05$ & $0.35 \pm 0.03$ & SSG, $\mathrm{H} \alpha+, \mathrm{X}$-ray \\
\hline 13438 & 154.39829 & -46.41403 & 17.17 & 5.9348 & \pm 0.0003 & $0.02 \pm 0.03$ & $36.6 \pm 0.9$ & $0.82 \pm 0.05$ & $0.35 \pm 0.03$ & SSG, H $\alpha, X$-ray \\
\hline 15138 & 154.38440 & -46.41622 & 18.14 & 6.393 & \pm 0.002 & $0.05 \pm 0.09$ & $18.8 \pm 2.3$ & $0.79 \pm 0.05$ & $0.16 \pm 0.03$ & bimodal \\
\hline 11366 & 154.41408 & -46.41498 & 19.08 & 8.381 & \pm 0.002 & $0.26 \pm 0.09$ & $43.5 \pm 4.3$ & $0.72 \pm 0.05$ & $0.44 \pm 0.06$ & \\
\hline 23220 & 154.40234 & -46.41006 & 17.05 & 8.428 & \pm 0.002 & $0.08 \pm 0.07$ & $12.9 \pm 1.0$ & $1.20 \pm 0.05$ & $0.15 \pm 0.02$ & BSS \\
\hline 25132 & 154.38563 & -46.41084 & 18.28 & 8.457 & \pm 0.002 & $0.07 \pm 0.08$ & $33.4 \pm 1.9$ & $0.78 \pm 0.05$ & $0.34 \pm 0.04$ & \\
\hline 10799 & 154.41927 & -46.41501 & 18.09 & 8.999 & \pm 0.006 & $0.09 \pm 0.17$ & $9.1 \pm 2.0$ & $0.79 \pm 0.05$ & $0.08 \pm 0.02$ & bimodal \\
\hline 15222 & 154.38393 & -46.41161 & 18.77 & 9.963 & \pm 0.002 & $0.26 \pm 0.09$ & $22.8 \pm 1.9$ & $0.75 \pm 0.05$ & $0.22 \pm 0.03$ & \\
\hline 14749 & 154.38811 & -46.41873 & 17.03 & 10.006 & \pm 0.002 & $0.09 \pm 0.07$ & $42.9 \pm 1.5$ & $0.82 \pm 0.05$ & $0.53 \pm 0.04$ & SSG, $\mathrm{H} \alpha$, EA \\
\hline 10719 & 154.41981 & -46.42151 & 18.99 & 10.930 & \pm 0.004 & $0.03 \pm 0.05$ & $43.7 \pm 2.4$ & $0.74 \pm 0.05$ & $0.54 \pm 0.05$ & \\
\hline 12305 & 154.40665 & -46.41503 & 18.48 & 15.41 & \pm 0.02 & $0.46 \pm 0.14$ & $23.7 \pm 8.9$ & $0.77 \pm 0.05$ & $0.25 \pm 0.09$ & \\
\hline 23918 & 154.39586 & -46.40850 & 18.19 & 15.945 & \pm 0.007 & $0.04 \pm 0.07$ & $32.2 \pm 1.9$ & $0.79 \pm 0.05$ & $0.43 \pm 0.04$ & \\
\hline 10418 & 154.42208 & -46.42361 & 19.07 & 16.128 & \pm 0.005 & $0.11 \pm 0.11$ & $28.5 \pm 2.5$ & $0.72 \pm 0.05$ & $0.35 \pm 0.05$ & \\
\hline 13684 & 154.39605 & -46.42072 & 20.00 & 16.73 & \pm 0.02 & $0.26 \pm 0.14$ & $23.6 \pm 3.4$ & $0.64 \pm 0.05$ & $0.25 \pm 0.05$ & bimodal \\
\hline 11405 & 154.41357 & -46.41997 & 17.25 & 17.219 & \pm 0.006 & $0.42 \pm 0.08$ & $14.1 \pm 1.4$ & $0.82 \pm 0.05$ & $0.15 \pm 0.02$ & SSG, $\mathrm{H} \alpha$ \\
\hline 10274 & 154.42387 & -46.41778 & 16.98 & 18.942 & \pm 0.006 & $0.32 \pm 0.05$ & $24.4 \pm 1.3$ & $1.20 \pm 0.05$ & $0.40 \pm 0.03$ & BSS \\
\hline 12370 & 154.40612 & -46.41486 & 19.46 & 19.46 & \pm 0.02 & $0.16 \pm 0.12$ & $21.8 \pm 2.7$ & $0.70 \pm 0.05$ & $0.26 \pm 0.05$ & \\
\hline 23019 & 154.40364 & -46.40831 & 18.70 & 25.76 & \pm 0.04 & $0.15 \pm 0.15$ & $13.3 \pm 1.9$ & $0.75 \pm 0.05$ & $0.17 \pm 0.03$ & \\
\hline 23847 & 154.39665 & -46.40951 & 16.12 & 26.70 & \pm 0.03 & $0.13 \pm 0.17$ & $6.1 \pm 0.9$ & $0.83 \pm 0.05$ & $0.08 \pm 0.02$ & bimodal \\
\hline 14837 & 154.38719 & -46.42433 & 19.12 & 32.37 & \pm 0.05 & $0.43 \pm 0.18$ & $16.0 \pm 2.0$ & $0.71 \pm 0.05$ & $0.20 \pm 0.04$ & \\
\hline 12706 & 154.40336 & -46.42295 & 18.05 & 34.11 & \pm 0.04 & $0.27 \pm 0.10$ & $12.8 \pm 1.3$ & $0.80 \pm 0.05$ & $0.18 \pm 0.03$ & \\
\hline 12871 & 154.40234 & -46.41310 & 20.35 & 38.2 & \pm 0.2 & $0.09 \pm 0.14$ & $18.3 \pm 2.7$ & $0.61 \pm 0.05$ & $0.27 \pm 0.11$ & bimodal \\
\hline 12341 & 154.40620 & -46.42160 & 17.18 & 38.92 & \pm 0.02 & $0.02 \pm 0.04$ & $22.6 \pm 0.7$ & $0.82 \pm 0.05$ & $0.41 \pm 0.03$ & \\
\hline 21659 & 154.41506 & -46.41032 & 17.29 & 39.52 & \pm 0.02 & $0.49 \pm 0.04$ & $27.7 \pm 2.2$ & $0.82 \pm 0.05$ & $0.45 \pm 0.05$ & \\
\hline 13357 & 154.39872 & -46.41345 & 18.08 & 42.16 & \pm 0.03 & $0.24 \pm 0.07$ & $23.1 \pm 1.2$ & $0.79 \pm 0.05$ & $0.42 \pm 0.04$ & \\
\hline 12308 & 154.40673 & -46.41448 & 17.67 & 47.39 & \pm 0.06 & $0.07 \pm 0.11$ & $29.4 \pm 2.5$ & $0.81 \pm 0.05$ & $0.64 \pm 0.09$ & \\
\hline 12082 & 154.40831 & -46.42402 & 18.09 & 47.88 & \pm 0.09 & $0.26 \pm 0.17$ & $13.0 \pm 2.1$ & $0.79 \pm 0.05$ & $0.21 \pm 0.05$ & \\
\hline 22700 & 154.40637 & -46.41026 & 18.38 & 53.5 & \pm 0.2 & $0.15 \pm 0.18$ & $10.6 \pm 1.7$ & $0.77 \pm 0.05$ & $0.18 \pm 0.04$ & \\
\hline 13221 & 154.39961 & -46.41524 & 18.49 & 53.7 & \pm 0.3 & $0.12 \pm 0.19$ & $13.3 \pm 2.3$ & $0.77 \pm 0.05$ & $0.23 \pm 0.05$ & \\
\hline 13669 & 154.39623 & -46.42264 & 17.45 & 53.8 & \pm 0.1 & $0.13 \pm 0.15$ & $11.7 \pm 1.9$ & $0.82 \pm 0.05$ & $0.21 \pm 0.04$ & \\
\hline 24738 & 154.38877 & -46.41100 & 18.02 & 55.44 & \pm 0.09 & $0.19 \pm 0.10$ & $15.5 \pm 1.7$ & $0.80 \pm 0.05$ & $0.29 \pm 0.04$ & \\
\hline 25266 & 154.38429 & -46.40955 & 18.06 & 55.5 & \pm 0.1 & $0.07 \pm 0.11$ & $13.7 \pm 2.0$ & $0.79 \pm 0.05$ & $0.25 \pm 0.05$ & \\
\hline 13002 & 154.40101 & -46.41437 & 18.29 & 56.4 & \pm 0.2 & $0.53 \pm 0.20$ & $7.1 \pm 1.9$ & $0.78 \pm 0.05$ & $0.10 \pm 0.03$ & bimodal \\
\hline 14889 & 154.38656 & -46.42310 & 18.67 & 56.71 & \pm 0.06 & $0.39 \pm 0.15$ & $27.8 \pm 8.5$ & $0.76 \pm 0.05$ & $0.56 \pm 0.19$ & \\
\hline 14972 & 154.38583 & -46.41625 & 18.02 & 69.2 & \pm 0.2 & $0.18 \pm 0.13$ & $12.2 \pm 1.7$ & $0.80 \pm 0.05$ & $0.23 \pm 0.05$ & \\
\hline 13756 & 154.39561 & -46.41871 & 18.52 & 71.3 & \pm 0.3 & $0.72 \pm 0.16$ & $32.3 \pm 20.0$ & $0.77 \pm 0.05$ & $0.53 \pm 0.33$ & \\
\hline 14743 & 154.38785 & -46.41935 & 18.20 & 74.3 & \pm 0.4 & $0.36 \pm 0.12$ & $20.5 \pm 2.4$ & $0.79 \pm 0.05$ & $0.43 \pm 0.08$ & \\
\hline 23000 & 154.40401 & -46.39918 & 17.61 & 75.3 & \pm 0.3 & $0.20 \pm 0.21$ & $6.3 \pm 1.2$ & $0.81 \pm 0.05$ & $0.12 \pm 0.03$ & \\
\hline 13522 & 154.39741 & -46.41123 & 18.09 & 108.2 & \pm 0.5 & $0.28 \pm 0.09$ & $17.7 \pm 2.3$ & $0.80 \pm 0.05$ & $0.44 \pm 0.08$ & \\
\hline 12904 & 154.40185 & -46.41983 & 16.07 & 110.3 & \pm 0.3 & $0.04 \pm 0.06$ & $11.6 \pm 0.7$ & $1.20 \pm 0.05$ & $0.35 \pm 0.03$ & BSS, $V \sin i$ \\
\hline 14700 & 154.38856 & -46.41702 & 17.71 & 112.3 & \pm 0.4 & $0.18 \pm 0.18$ & $11.3 \pm 2.0$ & $0.81 \pm 0.05$ & $0.26 \pm 0.06$ & bimodol \\
\hline 13355 & 154.39867 & -46.41374 & 19.93 & 115.1 & \pm 0.6 & $0.63 \pm 0.12$ & $34.9 \pm 10.0$ & $0.67 \pm 0.05$ & $0.82 \pm 0.22$ & \\
\hline 6324 & 154.38867 & -46.42641 & 15.64 & 120.5 & \pm 0.3 & $0.57 \pm 0.12$ & $14.2 \pm 4.8$ & $0.83 \pm 0.05$ & $0.29 \pm 0.08$ & \\
\hline 23175 & 154.40264 & -46.40494 & 15.07 & 129 & \pm 2 & $0.12 \pm 0.20$ & $5.2 \pm 1.4$ & $0.83 \pm 0.05$ & $0.12 \pm 0.04$ & \\
\hline 12927 & 154.40180 & -46.41472 & 19.07 & 143.4 & \pm 0.6 & $0.37 \pm 0.09$ & $14.1 \pm 1.5$ & $0.72 \pm 0.05$ & $0.33 \pm 0.05$ & \\
\hline 11300 & 154.41459 & -46.41824 & 17.00 & 163 & \pm 2 & $0.41 \pm 0.12$ & $17.3 \pm 4.4$ & $0.82 \pm 0.05$ & $0.48 \pm 0.13$ & \\
\hline
\end{tabular}

Notes. The Keplerian parameters were calculated with THE JOKER. The table is sorted by period. ACS Id: Identifier in the catalogue of NGC 320 in the ACS globular cluster survey (Sarajedini et al. 2007). BH1: Published black hole in Giesers et al. (2018). BHC: Star with a companion that could be a black hole (see Sect. 7.3). BSS: blue straggler star (see Sect. 7.1). Bimodal: The posterior sampling of the periods found by THE JOKER shows two modes. EA: Detached eclipsing binary (Clement 2017). EW: Contact eclipsing binary (Kaluzny et al. 2016). H $\alpha$ : Compared to our best fitting model spectrum (and to a well fitted $\mathrm{H} \beta$ line) several spectra of this star show a partially filled-in $\mathrm{H} \alpha$ absorption line (absorption is not as deep as expected). $\mathrm{H} \alpha+$ : Star showing real $\mathrm{H} \alpha$ emission in several MUSE spectra. NSC: Star with a companion that could be a neutron star. SSG: Sub-subgiant star (see Sect. 7.2). $V \sin i$ : star with measured spin velocity according to Simunovic \& Puzia (2014) (see Sect. 7.1). X-ray: Star listed as X-ray source in the November 2017 pre-release of the Chandra Source Catalog Release 2.0 (Evans et al. 2010). 
Chapter 2. Publications

A\&A 632, A3 (2019)

Table A.2. continued.

\begin{tabular}{|c|c|c|c|c|c|c|c|c|c|c|}
\hline ACS Id & $\begin{array}{l}\text { RA } \\
\left({ }^{\circ}\right)\end{array}$ & $\begin{array}{l}\text { Dec } \\
\left({ }^{\circ}\right)\end{array}$ & $\begin{array}{l}\text { Mag. } \\
\text { F606W }\end{array}$ & & iod & Eccentricity & $\begin{array}{c}\text { Amplitude } \\
\left(\mathrm{km} \mathrm{s}^{-1}\right)\end{array}$ & $\begin{array}{c}\text { Vis. mass } \\
\left(M_{\odot}\right)\end{array}$ & $\begin{array}{l}\text { Invis. min. mass } \\
\quad\left(M_{\odot} \sin i\right)\end{array}$ & Comment \\
\hline 12560 & 154.40454 & -46.41537 & 17.57 & 167.01 & \pm 0.09 & $0.61 \pm 0.02$ & $72.3 \pm 1.7$ & $0.81 \pm 0.05$ & $4.53 \pm 0.21$ & BH1 \\
\hline 4513 & 154.40662 & -46.42597 & 17.68 & 172 & \pm 2 & $0.07 \pm 0.12$ & $11.4 \pm 1.6$ & $0.81 \pm 0.05$ & $0.32 \pm 0.06$ & \\
\hline 22751 & 154.40602 & -46.40024 & 14.35 & 172.6 & \pm 0.6 & $0.03 \pm 0.04$ & $10.5 \pm 0.7$ & $0.83 \pm 0.05$ & $0.30 \pm 0.03$ & \\
\hline 12658 & 154.40360 & -46.42207 & 15.40 & 176.0 & \pm 0.4 & $0.07 \pm 0.05$ & $13.4 \pm 0.7$ & $0.83 \pm 0.05$ & $0.40 \pm 0.04$ & \\
\hline 14355 & 154.39107 & -46.41961 & 17.15 & 191 & \pm 3 & $0.06 \pm 0.15$ & $11.1 \pm 1.5$ & $1.20 \pm 0.05$ & $0.41 \pm 0.07$ & BSS, bimodal \\
\hline 12438 & 154.40560 & -46.41283 & 17.22 & 197 & \pm 2 & $0.06 \pm 0.10$ & $8.2 \pm 1.5$ & $0.82 \pm 0.05$ & $0.23 \pm 0.05$ & \\
\hline 23276 & 154.40164 & -46.40921 & 17.51 & 204.2 & \pm 1.0 & $0.89 \pm 0.06$ & $21.2 \pm 10.6$ & $0.82 \pm 0.05$ & $0.30 \pm 0.12$ & \\
\hline 23452 & 154.40018 & -46.40326 & 16.79 & 206 & \pm 2 & $0.06 \pm 0.08$ & $7.2 \pm 0.9$ & $0.82 \pm 0.05$ & $0.20 \pm 0.03$ & \\
\hline 12975 & 154.40134 & -46.42046 & 18.67 & 224 & \pm 4 & $0.07 \pm 0.11$ & $11.5 \pm 1.5$ & $0.77 \pm 0.05$ & $0.35 \pm 0.06$ & \\
\hline 13391 & 154.39790 & -46.42029 & 18.71 & 225 & \pm 6 & $0.24 \pm 0.25$ & $9.8 \pm 2.2$ & $0.76 \pm 0.05$ & $0.28 \pm 0.08$ & \\
\hline 23381 & 154.40051 & -46.40725 & 17.24 & 262 & \pm 2 & $0.11 \pm 0.15$ & $12.1 \pm 1.7$ & $0.82 \pm 0.05$ & $0.42 \pm 0.07$ & \\
\hline 23889 & 154.39636 & -46.40059 & 17.64 & 282 & \pm 5 & $0.33 \pm 0.14$ & $13.2 \pm 2.0$ & $0.81 \pm 0.05$ & $0.43 \pm 0.09$ & \\
\hline 11945 & 154.40948 & -46.41194 & 17.44 & 284 & \pm 8 & $0.18 \pm 0.20$ & $9.8 \pm 2.7$ & $1.20 \pm 0.05$ & $0.40 \pm 0.15$ & BSS \\
\hline 14055 & 154.39350 & -46.41191 & 17.27 & 286 & \pm 5 & $0.17 \pm 0.18$ & $9.0 \pm 1.6$ & $0.82 \pm 0.05$ & $0.29 \pm 0.06$ & \\
\hline 13816 & 154.39521 & -46.41600 & 16.11 & 302 & \pm 5 & $0.17 \pm 0.20$ & $3.9 \pm 1.0$ & $0.83 \pm 0.05$ & $0.11 \pm 0.03$ & \\
\hline 23848 & 154.39645 & -46.40946 & 16.52 & 323 & \pm 2 & $0.48 \pm 0.08$ & $15.6 \pm 1.5$ & $1.20 \pm 0.05$ & $0.67 \pm 0.11$ & BSS \\
\hline 12173 & 154.40785 & -46.41662 & 17.77 & 340 & \pm 6 & $0.47 \pm 0.24$ & $20.0 \pm 7.9$ & $0.81 \pm 0.05$ & $0.80 \pm 0.30$ & bimodal \\
\hline 13069 & 154.40069 & -46.41497 & 17.01 & 375 & \pm 3 & $0.14 \pm 0.19$ & $9.3 \pm 2.7$ & $0.82 \pm 0.05$ & $0.35 \pm 0.14$ & \\
\hline 12932 & 154.40168 & -46.41370 & 17.81 & 386 & \pm 9 & $0.48 \pm 0.20$ & $6.5 \pm 1.9$ & $0.81 \pm 0.05$ & $0.19 \pm 0.07$ & \\
\hline 11831 & 154.41014 & -46.42258 & 13.88 & 493 & \pm 11 & $0.07 \pm 0.14$ & $6.4 \pm 0.9$ & $0.83 \pm 0.05$ & $0.25 \pm 0.04$ & \\
\hline 24135 & 154.39382 & -46.41077 & 18.12 & 509 & \pm 20 & $0.51 \pm 0.30$ & $8.2 \pm 6.7$ & $0.79 \pm 0.05$ & $0.28 \pm 0.18$ & \\
\hline 13587 & 154.39637 & -46.42422 & 18.52 & 537 & \pm 21 & $0.13 \pm 0.15$ & $10.6 \pm 1.8$ & $1.20 \pm 0.05$ & $0.59 \pm 0.14$ & BSS \\
\hline 11317 & 154.41447 & -46.41358 & 15.77 & 602 & \pm 12 & $0.25 \pm 0.13$ & $9.4 \pm 1.4$ & $0.83 \pm 0.05$ & $0.42 \pm 0.07$ & \\
\hline 13874 & 154.39476 & -46.42003 & 18.59 & 608 & \pm 27 & $0.30 \pm 0.30$ & $10.1 \pm 4.0$ & $0.77 \pm 0.05$ & $0.43 \pm 0.20$ & \\
\hline 11779 & 154.41062 & -46.42064 & 17.43 & 620 & \pm 35 & $0.13 \pm 0.21$ & $8.2 \pm 1.7$ & $0.82 \pm 0.05$ & $0.36 \pm 0.10$ & \\
\hline 15293 & 154.38311 & -46.42006 & 16.75 & 668 & \pm 20 & $0.48 \pm 0.10$ & $11.3 \pm 1.8$ & $0.82 \pm 0.05$ & $0.49 \pm 0.10$ & \\
\hline 5132 & 154.40034 & -46.42585 & 20.17 & 764 & \pm 11 & $0.28 \pm 0.16$ & $36.3 \pm 8.4$ & $0.64 \pm 0.05$ & $4.40 \pm 2.82$ & BHC, bimodal \\
\hline 13782 & 154.39552 & -46.41252 & 17.26 & 846 & \pm 61 & $0.11 \pm 0.20$ & $8.2 \pm 1.9$ & $0.82 \pm 0.05$ & $0.42 \pm 0.12$ & \\
\hline 25211 & 154.38487 & -46.40768 & 17.41 & 988 & \pm 75 & $0.11 \pm 0.16$ & $8.7 \pm 1.7$ & $1.20 \pm 0.05$ & $0.59 \pm 0.14$ & BSS \\
\hline 12828 & 154.40192 & -46.42344 & 18.09 & 1075 & \pm 37 & $0.05 \pm 0.08$ & $13.2 \pm 1.4$ & $0.80 \pm 0.05$ & $0.90 \pm 0.16$ & \\
\hline 11131 & 154.41636 & -46.41623 & 17.28 & 1627 & \pm 222 & $0.31 \pm 0.17$ & $8.2 \pm 1.6$ & $0.82 \pm 0.05$ & $0.54 \pm 0.13$ & bimodal \\
\hline 23215 & 154.40267 & -46.39830 & 18.73 & 1997 & \pm 304 & $0.08 \pm 0.14$ & $16.3 \pm 2.1$ & $0.76 \pm 0.05$ & $1.82 \pm 0.39$ & NSC \\
\hline 13154 & 154.39996 & -46.41225 & 18.17 & 2773 & \pm 1009 & $0.16 \pm 0.19$ & $10.1 \pm 4.1$ & $0.79 \pm 0.05$ & $0.94 \pm 0.94$ & \\
\hline 13808 & 154.39505 & -46.41923 & 15.23 & 3001 & \pm 1005 & $0.11 \pm 0.22$ & $3.2 \pm 1.4$ & $0.83 \pm 0.05$ & $0.21 \pm 0.13$ & \\
\hline
\end{tabular}

A3, page 20 of 20 


\title{
The binary content of multiple populations in NGC 3201
}

\author{
S. Kamann ${ }^{1}$, B. Giesers ${ }^{2}$, N. Bastian ${ }^{1}$, J. Brinchmann ${ }^{3,4}$, S. Dreizler ${ }^{2}$, F. Göttgens ${ }^{2}$, T.-O. Husser ${ }^{2}$, M. Latour $^{2}$, \\ P. M. Weilbacher ${ }^{5}$, and L. Wisotzki ${ }^{5}$ \\ 1 Astrophysics Research Institute, Liverpool John Moores University, 146 Brownlow Hill, Liverpool L3 5RF, UK \\ e-mail: s.kamann@ljmu.ac.uk \\ 2 Institute for Astrophysics, Georg-August-Universität Göttingen, Friedrich-Hund-Platz 1, 37077 Göttingen, Germany \\ 3 Instituto de Astrofísica e Ciências do Espaço, Universidade do Porto, CAUP, Rua das Estrelas, 4150-762 Porto, Portugal \\ ${ }^{4}$ Leiden Observatory, Leiden University, PO Box 9513, 2300 RA Leiden, The Netherlands \\ ${ }^{5}$ Leibniz-Institute for Astrophysics, An der Sternwarte 16, 14482 Potsdam, Germany \\ Received 4 October 2019 / Accepted 5 February 2020
}

\section{ABSTRACT}

We investigate the binary content of the two stellar populations that coexist in the globular cluster NGC 3201 . Previous studies of binary stars in globular clusters have reported higher binary fractions in their first populations (P1, having field-like abundances) compared to their second populations ( $\mathrm{P} 2$, having anomalous abundances). This is interpreted as evidence for the latter forming more centrally concentrated. In contrast to previous studies, our analysis focusses on the cluster centre, where comparable binary fractions between the populations are predicted because of short relaxation times. However, we find that even in the centre of NGC 3201, the observed binary fraction of $\mathrm{P} 1$ is higher $(23.1 \pm 6.2) \%$ compared to $(8.2 \pm 3.5) \%$ in $\mathrm{P} 2$. Our results are difficult to reconcile with a scenario in which the populations only differ in their initial concentrations, but instead suggests that the populations also formed with different fractions of binary stars.

Key words. binaries: spectroscopic - techniques: radial velocities - globular clusters: individual: NGC 3201 - stars: abundances

\section{Introduction}

One of the lesser studied aspects of the multiple populations (also known as abundance anomalies, see Bastian \& Lardo 2018, for a review) phenomena in massive stellar clusters is the role of stellar binarity. This is due to the overall relatively low binary fractions in globular clusters (GCs; e.g. Ji \& Bregman 2015) and the fact that it is difficult to separate out the binaries from (apparent) single stars in colour-magnitude diagrams for each of the populations (i.e. the "normal" and "anomalous" stars; P1 and P2) as the sequences overlap. Instead, we must carry out intensive spectroscopic time-series analyses of a representative sample of stars from each population to search for radial velocity variations.

The most comprehensive survey using this technique, to date, is that of Lucatello et al. (2015) who monitored 968 red giant branch (RGB) stars in ten Milky Way ancient GCs. From this large sample these authors find 21 binary stars and when separating their sample into $\mathrm{P} 1$ and $\mathrm{P} 2$ stars, find binary fractions of $4.9 \%$ and $1.2 \%$ for each population, respectively. In addition, Dalessandro et al. (2018) report a higher binary fraction in P1 of the GC NGC 6362, $14 \%$ compared to $<1 \%$ in P2.

Such differences can be explained in terms of the formation environment of the stars; the P2 stars (lower binary fraction) form and initially evolve in a much denser environment, which would destroy many of the primordial binaries (e.g. Hong et al. 2016). An initially more concentrated P2 is a common feature of essentially all scenarios put forward to explain multiple populations and appears to be in agreement with the observed density profiles and kinematics of the populations in most Galactic GCs today (e.g. Lardo et al. 2011; Richer et al. 2013; Bellini et al. 2015; Dalessandro et al. 2019).
As a consequence of the fibre-based observations, the targets for the study of Lucatello et al. (2015) are preferentially located in the outer regions of the clusters. Most GCs show a trend of increasing binary fractions towards the cluster centres (e.g. Milone et al. 2012), which is thought to be due to mass segregation. On the other hand, dynamical processes lowering the binary fractions, such as binary disruption or ejection from the cluster, occur more frequently near to the cluster centres. Therefore, the binary statistics near to the cluster centres may not follow those in the cluster outskirts. Using $N$-body simulations, Hong et al. $(2015,2016)$ find that the binary fractions of $\mathrm{P} 2$ are expected to be comparable or even larger than those of P1 inside the halflight radii of the clusters if P2 formed centrally concentrated.

In the present work we explicitly test these predictions, using the time series VLT/MUSE observations of NGC 3201, stretching over more than four years, presented in Giesers et al. (2019), which focus on the region inside the core radius $\left(r_{\mathrm{c}}=1.3^{\prime} \equiv\right.$ 1.85; Harris 1996) of the cluster. RGB stars from the different populations are found using a UV-optical "chromosome map" (Milone et al. 2017), which is highly efficient in separating the populations, largely based on their $N$ abundance differences (Lardo et al. 2018).

\section{Data}

NGC 3201 has been observed as part of the MUSE survey of Galactic GCs (see Kamann et al. 2018), a large GTO programme targeting the central regions of massive star clusters. To facilitate the detection and characterisation of binary stars, repeated observations of five pointings, covering approximately the central $2^{\prime} \times 2^{\prime}$ of the cluster, were performed from November 2014 to 

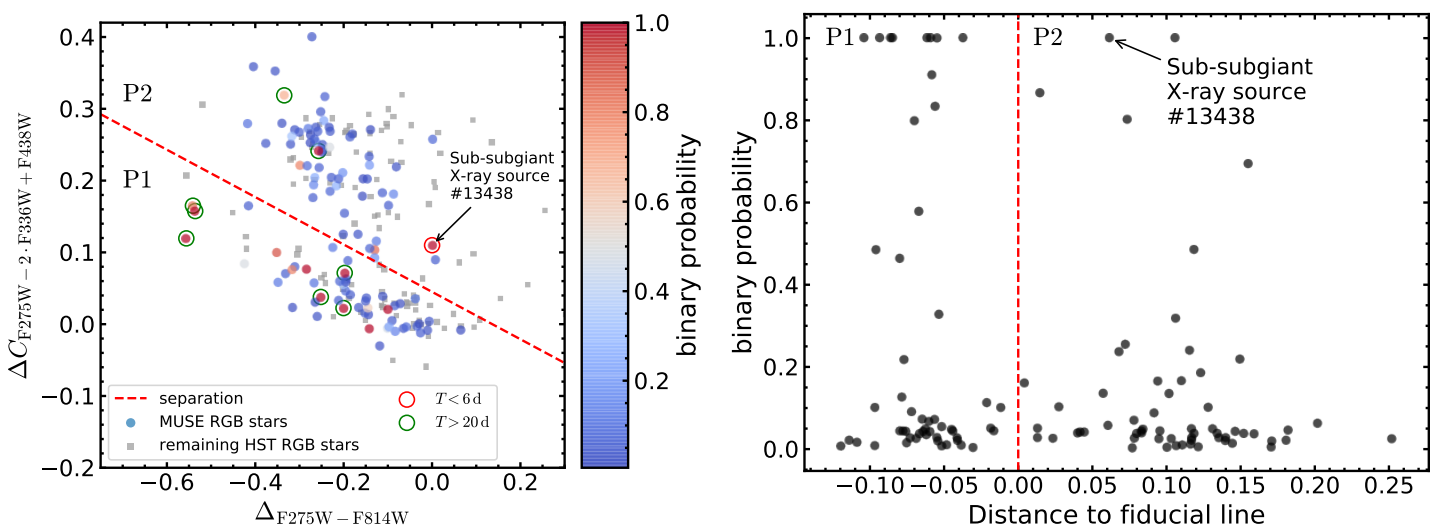

Fig. 1. Left: distribution of red giant stars in NGC 3201 in pseudo-colour space, the so-called chromosome map. The full sample of stars obtained from the HST photometry of Piotto et al. (2015) is shown as small grey dots. Stars available in the MUSE sample are highlighted and colour-coded according to their probability of being in a binary system. For the sub-sample of MUSE sources with known Keplerian parameters, coloured rings indicate the orbital period $T$. The dashed red line illustrates our separation into P1 and P2 stars. Right: the binary probability of the stars in the MUSE sample is shown as a function of the distance of a star perpendicular to the line separating P1 and P2 (i.e. the dashed red line in the left panel).

May 2019. The data analysis, including the detection and characterisation of binaries, are described in Giesers et al. (2019). For each of the 3553 stars studied, the authors provided a probability that the star shows radial velocity variations. The radial velocities of the stars with at least five observations and with a probability higher than $50 \%$ of being variable were further analysed with THE JOKER (Price-Whelan et al. 2018), resulting in a subset of 95 stars with unique Keplerian orbit solutions.

To split up the two stellar populations that have previously been identified in NGC 3201, we used the Hubble Space Telescope (HST) photometry from the survey of Piotto et al. (2015, see Nardiello et al. 2018). As outlined in Latour et al. (2019), this was done by creating a "chromosome map" from the red giant stars (see Milone et al. 2015), which is shown in Fig. 1. The separation of the two populations was performed following Milone et al. (2017) and is indicated by the red dashed line included in Fig. 1 (with P1 being below the fiducial line).

Finally, we identified the subset of stars from Giesers et al. (2019) for which the population could be determined. This resulted in a final sample of 113 stars, 52 in P1, and 61 in P2, which is presented in Table A.1. For the 17 out of 113 stars which had a probability $P>0.5$ of being in a binary and sufficient $(\geq 5)$ observations, we tried to determine the Keplerian orbit. This resulted in a subset of nine stars, for which an orbit solution is available. The orbital parameters of said stars are included in Table A.1. The remaining eight stars with $P>0.5$ have insufficient kinematical data to infer their Keplerian orbits.

\section{Results}

\subsection{Binaries across the chromosome map}

The distribution of binary stars across the chromosome map of NGC 3201 is shown in the left panel of Fig. 1. We colour-coded each star available in the sample of Giesers et al. (2019) by its probability to be in a binary system. Stars for which orbital solutions have been found are further highlighted according to their orbital period $T$. To better visualise possible differences between $\mathrm{P} 1$ and $\mathrm{P} 2$, we show the binary probability as a function of the A65, page 2 of 6 distance perpendicular to the fiducial line separating P1 and P2 in the right panel of Fig. 1

To infer the binary fractions in both populations, we followed Giesers et al. (2019) and obtain the fraction of stars with a binary probability of $P>0.5$ within each population. This leads to binary fractions of $(23.1 \pm 6.2) \%$ in $\mathrm{P} 1$ and $(8.2 \pm 3.5) \%$ in $\mathrm{P} 2$. The uncertainties tailored to both values take into account the limited sample sizes as well as the uncertainties stemming from the threshold in $P$ (see Giesers et al. 2019, for details). When calculating the binary fraction in $\mathrm{P} 2$, we included the sub-subgiant star highlighted in Fig. 1, which is in a much tighter orbit than the remaining binary systems (indicated by the coloured rings in Fig. 1). As discussed in Giesers et al. (2019), this star has an $\mathrm{X}$-ray counterpart and shows $\mathrm{H} \alpha$ emission. Hence it is plausible that this star is part of an accreting binary system, which would also impact its photometric properties and its location in the chromosome map. Excluding it from our calculation reduces the binary fraction of P2 to $(6.7 \pm 3.3) \%$. Averaged over both populations, we find a binary fraction of $(15.0 \pm 3.4) \%$, in good agreement with the discovery fraction of $(17.1 \pm 1.9) \%$ determined by Giesers et al. (2019).

\subsection{Origin of the observed binaries}

To study the origin of the observed binaries, we made use of the subsample with known orbital parameters. The fate of a binary in a $\mathrm{GC}$ is linked to its hardness $h$, that is the ratio of its internal energy $\tilde{E}$ to the average kinetic energy of the surrounding stars,

$h=|\tilde{E}| / m \sigma^{2}$

where $m$ is the typical stellar mass of a cluster member and $\sigma$ the velocity dispersion of the cluster. For a bound Keplerian orbit, the internal energy is given as

$\tilde{E}=-\frac{G m_{\mathrm{p}} m_{\mathrm{c}}}{2 a}$,

where $m_{\mathrm{p}}$ and $m_{\mathrm{c}}$ are the masses of the constituents and $a$ is the semi-major axis of the binary. In Table A.1, we provide the 
hardness for each binary with an orbit available. The values were calculated assuming an inclination of $i=90^{\circ}$ (i.e. the minimum possible companion mass $m_{\mathrm{c}}$ ). The mass of the primary (RGB) component, $m_{\mathrm{p}}$, was determined via comparison to an isochrone as described in Giesers et al. (2019). As cluster properties, we used $m=0.8 M_{\odot}$ and $\sigma=4.3 \mathrm{~km} \mathrm{~s}^{-1}$ (Baumgardt \& Hilker 2018). All systems are hard binaries with $h>1$, indicating that they can survive in NGC 3201 for a Hubble time. Their longevity can be confirmed by determining the expected lifetimes of the binary stars, $\tau=1 / B(\tilde{E})$, where $B(\tilde{E})$ is the probability of a binary being ionised (i.e. destroyed) in a gravitational encounter with a third cluster member, given as (Eq. (7.174) in Binney \& Tremaine 2008)

$B(\tilde{E})=\frac{8 \sqrt{\pi} G^{2} m^{3} \rho \sigma}{3^{3 / 2}|\tilde{E}|}\left(1+\frac{1}{5 h}\right)^{-1}\left[1+e^{\mathrm{h}}\right]^{-1}$

Evaluating Eq. (3) for a core density of $\rho=10^{2.72} M_{\odot} / \mathrm{pc}^{3}$ (Baumgardt \& Hilker 2018) yields lifetimes for all binary stars that exceed the age of NGC 3201 by several orders of magnitude.

We note that the companion masses $m_{2}$ and the semi-major axes $a$ used in the above calculations were derived under the assumption that the binaries are observed edge-on (i.e. at an inclination of $i=90 \mathrm{deg}$ ). While both quantities increase with decreasing inclinations, $m_{2}$ is more sensitive on $i$ than $a$ is, so that our hardness values can be considered as lower limits.

Finally, we stress that the probability to form hard binaries dynamically in a relatively low-density cluster such as NGC 3201 is very small. Using Eq. 7.176 from Binney \& Tremaine (2008), the formation rate of hard binaries per unit volume is given as

$C_{\mathrm{hb}}=0.74 \frac{G^{5} \rho^{3} m^{2}}{\sigma^{9}}$

Integrating Eq. (4) over the core of NGC 3201 (assuming $r_{\mathrm{c}}=$ 1.74; Baumgardt \& Hilker 2018) yields a total formation rate of $7 \times 10^{-6} \mathrm{Gyr}^{-1}$. Hence it is very likely that all binary stars that we observe in NGC 3201 are primordial.

\subsection{Impact of the companion}

Our determination of the binary fraction in each population is based on the assumption that the positions of the stars in the chromosome map are not altered by the presence of their companions. To verify this assumption, we used the binaries with known orbits and inferred the magnitude changes caused by their companions in the four HST filters underlying the chromosome map, F275W, F336W, F438W, and F814W. To this aim, we fetched an isochrone tailored to the properties of NGC $3201\left([\mathrm{Fe} / \mathrm{H}]=-1.59, E_{\mathrm{B}-\mathrm{V}}=0.24\right.$; Harris 1996) from the MIST database (Choi et al. 2016). We made the assumption that the companions are main-sequence stars and predicted their magnitudes $\operatorname{mag}_{\mathrm{c}}$ by selecting the isochrone points along the main sequence closest to their measured masses, $m_{\mathrm{c}} \sin i$ (cf. Table A.1). As the measured companion masses need to be corrected for the (unknown) orbit inclinations relative to the line of sight, we assumed different inclination angles $i$ and found the isochrone counterpart for each value of $m_{\mathrm{c}}$. At each inclination, we calculated the corrected magnitude mag $_{\mathrm{p}}$ of the RGB star in the four relevant filters, according to

$\operatorname{mag}_{\mathrm{p}}=\operatorname{mag}_{\text {tot }}-2.5 \log _{10}\left(1-10^{-0.4\left(\mathrm{mag}_{\mathrm{c}}-\mathrm{mag}_{\text {tot }}\right)}\right)$,

where mag $_{\text {tot }}$ is the measured magnitude of the system in the considered filter. Then we predicted the actual location of each

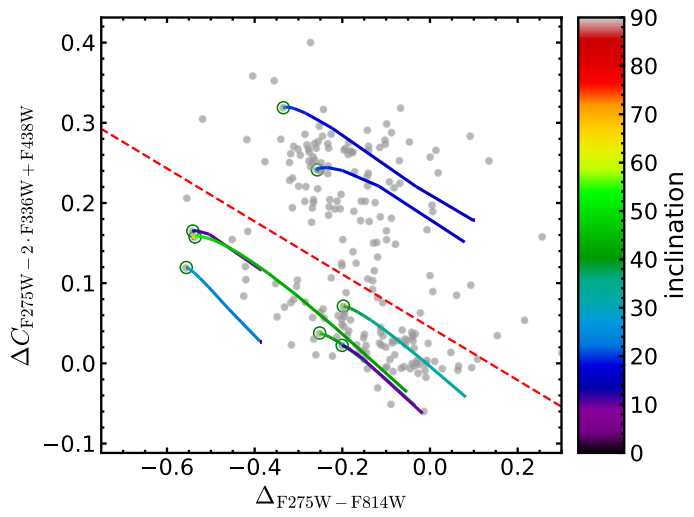

Fig. 2. Impact of binary companions on the positions of RGB stars in the chromosome map of NCG 3201. For each star from our sample with known orbit, the true position of the RGB star after subtracting the contribution of a main-sequence companion, as a function of orbit inclination, is shown. The grey points and dashed red line are the same as in the left panel of Fig. 1 .

RGB star in the chromosome map. We stopped when subtracting the contribution of a fiducial companion resulted in a predicted position that was off by more than $0.1 \mathrm{mag}$ from the red edge of the RGB in either $(F 275 W-F 814 W)$ colour or $(F 275 W-2$. $F 336 W+F 438 W$ ) pseudo-colour.

We summarise the outcome of this test in Fig. 2. It shows the predicted position of the RGB star in the chromosome map as a function of the inclination for each binary in our sample with a known orbit. Figure 2 shows that P2 stars in a binary with a main-sequence star are very unlikely to appear as P1 stars (and vice versa), as the companion tends to shift the binary in a direction parallel to the fiducial line separating the populations.

We further find that the companion needs to be massive enough to appear close to the main-sequence turn-off to have a significant effect. Figure 2 shows that for all of the sources in our sample, their orbits would need to be observed at low inclinations, $i \lesssim 40^{\circ}$, in that case, because our minimum masses are significantly below the expected turn-off mass of NGC 3201 $\left(m_{\mathrm{TO}} \sim 0.8 M_{\odot} ;\right.$ cf. Table A.1). Under the assumption of randomly orientated orbits, we can estimate the probabilities to observe the systems at or below the inclinations where the companions have a measurable effect on the observed positions in the chromosome map. We find probabilities between $<1 \%$ and about $35 \%$ for the individual systems. Considering the sum of probabilities for the eight stars, we expect about one star among the eight to have been measurably shifted by its companion. We note that these probabilities do not account for the selection bias of radial velocity studies, which are more sensitive to edge-on orbits, and hence can be considered as upper limits (see discussion in Carroll \& Ostlie 2006).

We also considered the possibility of white-dwarf companions, as they may have a stronger impact on the $F 275 \mathrm{~W}$ flux However, in the photometry of Nardiello et al. (2018), we find only $10-15$ white dwarf candidates with a $F 275 W$ magnitude within 2 mag of the main-sequence turn-off.

\section{Discussion}

At first glance, our finding of a lower binary fraction in P2 than in P1 agrees with previous studies on the binary content 
Chapter 2. Publications

A\&A 635, A65 (2020)

of multiple populations (Lucatello et al. 2015; Dalessandro et al. 2018). This trend was attributed to the P2 stars forming centrally concentrated, resulting in a higher rate of binary ionisation and ejection. However, in contrast to earlier studies, our observations focus on the dense cluster core. In the simulations of Hong et al. (2015, 2016), the overabundance in P1 binaries typically only develops outside the half-light radii of the simulated clusters, whereas the trend disappears or even reverses inside of this radius ${ }^{1}$. The observation by Dalessandro et al. (2018) that the discrepancy in the observed velocity dispersions between P1 and P2 stars in NGC 6362, which is attributed to the overabundance of P1 binaries, disappears towards the centre can also be interpreted as a hint about comparable central binary fractions.

Compared to the clusters simulated by Hong et al. (2015, 2016), where all stars were input with the same masses, NGC 3201 appears much more complex. One complication is the likely presence of a large population of stellar-mass black holes (Giesers et al. 2018, 2019; Askar et al. 2018), which is expected to have a strong impact on the evolution of NGC 3201. Owing to their masses, black holes can efficiently suppress the segregation of the binaries to the cluster centre. As the evolution of a binary population is governed by the interplay between mass segregation and their interactions with other stars, this marks an important difference compared to the existing simulations. Dedicated simulations using a realistic range of stellar masses will be an important step forward towards understanding the evolution of binary stars in multiple populations.

A possible explanation for our results is that $\mathrm{P} 2$ had different binary properties than P1 upon formation, for example a lower primordial binary fraction or a different distribution of semimajor axes. Most formation scenarios predict P2 stars to form while at least part of the $\mathrm{P} 1$ population is already in place. It seems likely that such vastly different formation environments had an impact on the properties of the primordial binaries in $\mathrm{P} 2$. Future hydrodynamical simulations of cluster formation may be able to investigate this further.

We note that some of the detected P1 binaries appear in a region of the chromosome map that is termed the extended P1 (e.g. Lardo et al. 2018), that is to the top left of the bulk of P1 stars. As shown by for example Cabrera-Ziri et al. (2019), extended P1 stars show no differences in their abundances of $\mathrm{C}$, $\mathrm{N}, \mathrm{O}, \mathrm{Na}, \mathrm{Mg}$, or Al compared to normal P1 stars. Marino et al. (2019) argue that binaries could be responsible for creating extended P1 stars in NGC 3201 (also see Martins et al. 2020). As our analysis of Sect. 3.3 shows, normal P1 stars in binary systems with main-sequence stars close to the turn-off can be shifted into the extended branch. However, it appears unlikely that this scenario is responsible for all of the stars observed along the extended P1. The binary systems with unique orbital solutions would need to be observed at rather unlikely inclination angles for the companions to produce noticeable shifts. In addition, a number of extended P1 stars do not show any signs of variability in our sample. Nevertheless, extending our analysis to other clusters with a pronounced extended P1, such as NGC 2808, appears to be a very promising step in studying the impact of binary stars on the distribution of stars across chromosome maps.

Finally, we note that in comparison to Marino et al. (2019), our extended P1 extends to smaller values of $\Delta_{F 275 W-F 814 W}$. Upon removal of the stars with $\Delta_{F 275 W-F 814 W} \lesssim-0.4$, which were not considered in the work by Marino et al. (2019), our P1 binary fraction reduces to $19.1 \pm 5.7 \%$. Hence our main conclusion of a higher binary fraction in P1 than in P2 does not depend on the exact definition of which stars belong to P1.

Acknowledgements. SK and NB gratefully acknowledge funding from a European Research Council consolidator grant (ERC-CoG-646928- Multi-Pop). BG, SD, TOH, an ML acknowledge funding from the Deutsche Forschungsgen SD, ( 05A17MGA, 05A14BAC, and 05A17BAA. NB gratefully acknowledges financial support from the Royal Society in the form of a University Research Fellowhip. JB acknowledges support by FCT/MCTES through national funds (PIDDAC) by grant UID/FIS/04434/2019 and through Investigador FCT Contract No. IF/01654/2014/CP1215/CT0003.

\section{References}

Anderson, J., Sarajedini, A., Bedin, L. R., et al. 2008, AJ, 135, 2055 Askar, A., Arca Sedda, M., \& Giersz, M. 2018, MNRAS, 478, 1844 Bastian, N., \& Lardo, C. 2018, ARA\&A, 56, 83 Baumgardt, H., \& Hilker, M. 2018, MNRAS, 478, 1520 Bellini, A., Vesperini, E., Piotto, G., et al. 2015, ApJ, 810, L13 Binney, J., \& Tremaine, S. 2008, Galactic Dynamics: Second Edition (Princeton: Princeton University Press

Cabrera-Ziri, I., Lardo, C., \& Mucciarelli, A. 2019, MNRAS, 485, 4128

Carroll, B. W., \& Ostlie, D. A. 2006, An Introduction to Modern Astrophysics and Cosmology (San Francisco: Addison-Wesley)

Choi, J., Dotter, A., Conroy, C., et al. 2016, ApJ, 823, 102

Dalessandro, E., Mucciarelli, A., Bellazzini, M., et al. 2018, ApJ, 864, 33 Dalessandro, E., Cadelano, M., Vesperini, E., et al. 2019, ApJ, 884, L24 Giesers, B., Dreizler, S., Husser, T.-O., et al. 2018, MNRAS, 475, L15 Giesers, B., Kamann, S., Dreizler, S., et al. 2019, A\&A, 632, A3

Harris, W. E. 1996, AJ, 112, 1487

Hong, J., Vesperini, E., Sollima, A., et al. 2015, MNRAS, 449, 629 Hong, J., Vesperini, E., Sollima, A., et al. 2016, MNRAS, 457, 4507 Ji, J., \& Bregman, J. N. 2015, ApJ, 807, 32

Kamann, S. Husser, T.-O., Dreizler, S., et al 2018, MNRAS, 473, 5591

Kamann, S., Husser, T.-O., Dreizler, S., et al. 2018, MNRAS, 473, 559

Lardo, C., Bellazzini, M., Pancino, E., et al. 2011, A\&A, 525, A

Lardo, C., Salaris, M., Bastian, N., et al. 2018, A\&A, 616, A168

Latour, M., Husser, T. O., Giesers, B., et al. 2019, A\&A, 631, A14 Lucatello, S., Sollima, A., Gratton, R., et al. 2015, A\&A, 584, A5 Marino, A. F., Milone, A. P., Sills, A., et al. 2019, ApJ, 887, 91 Martins, F., Morin, J., Charbonnel, C., Lardo, C., \& Chantereau, W. 2020, A\&A, $635, \mathrm{~A} 52$

Milone, A. P., Piotto, G., Bedin, L. R., et al. 2012, A\&A, 540, A16

Milone, A. P., Marino, A. F. Piotto, G., et al. 2015, MNRAS, 447, 927

Milone, A. P. Piotto,

Mil,

Nardiello, D., Libralato, M., Piotto, G., et al. 2018, MNRAS, 481, 3382
Piotto, G., Milone, A. P., Bedin, L. R., et al. 2015, AJ, 149, 91

Price-Whelan, A. M., Hogg, D. W., Rix, H.-W., et al. 2018, AJ, 156, 18 Richer, H. B., Heyl, J., Anderson, J., et al. 2013, ApJ, 771, L15

1 In contrast to Hong et al. $(2015,2016)$, we can only infer the binary fractions as a function of projected radius.

A65, page 4 of 6 
The binary content of multiple populations in NGC 3201

S. Kamann et al.: The binary content of multiple populations in NGC 3201

\section{Appendix A: Table}

Table A.1. Photometric and orbital properties of the binaries with unique Kepler solutions in the MUSE sample.

\begin{tabular}{|c|c|c|c|c|c|c|c|c|c|c|}
\hline ACS Id & $\Delta_{275,814}$ & $\Delta C_{275,336,438}$ & Pop. & $P_{\text {bin }}$ & $m_{\mathrm{p}} / M_{\odot}$ & $m_{\mathrm{c}} \sin i / M_{\odot}$ & $a / \mathrm{AU}^{(a)}$ & $e$ & $T / \mathrm{d}$ & $h$ \\
\hline 3092 & 0.064 & -0.008 & 1 & 0.006 & & & & & & \\
\hline 3248 & 0.000 & 0.257 & 2 & 0.065 & & & & & & \\
\hline 3795 & -0.142 & 0.222 & 2 & 0.188 & & & & & & \\
\hline 4121 & -0.424 & 0.084 & 1 & 0.486 & & & & & & \\
\hline 4125 & -0.320 & 0.251 & 2 & 0.027 & & & & & & \\
\hline 4566 & -0.225 & 0.107 & 1 & 0.103 & & & & & & \\
\hline 4698 & -0.243 & 0.317 & 2 & 0.049 & & & & & & \\
\hline 4853 & -0.119 & -0.030 & 1 & 0.019 & & & & & & \\
\hline 5281 & -0.348 & 0.059 & 1 & 0.103 & & & & & & \\
\hline 5461 & -0.201 & 0.125 & 2 & 0.030 & & & & & & \\
\hline 6228 & -0.195 & 0.063 & 1 & 0.047 & & & & & & \\
\hline 6560 & -0.127 & 0.116 & 2 & 0.105 & & & & & & \\
\hline 10705 & -0.311 & 0.271 & 2 & 0.024 & & & & & & \\
\hline 10741 & -0.265 & 0.031 & 1 & 0.011 & & & & & & \\
\hline 10753 & -0.065 & -0.005 & 1 & 0.028 & & & & & & \\
\hline 10968 & -0.262 & 0.253 & 2 & 0.242 & & & & & & \\
\hline 11180 & 0.007 & 0.090 & 2 & 0.044 & & & & & & \\
\hline 11203 & -0.265 & 0.204 & 2 & 0.239 & & & & & & \\
\hline 11273 & -0.168 & 0.125 & 2 & 0.029 & & & & & & \\
\hline 11281 & -0.130 & 0.104 & 2 & 0.866 & & & & & & \\
\hline 11294 & -0.196 & 0.047 & 1 & 0.070 & & & & & & \\
\hline 11305 & -0.085 & 0.026 & 1 & 0.050 & & & & & & \\
\hline 11306 & -0.082 & 0.219 & 2 & 0.023 & & & & & & \\
\hline 11317 & -0.252 & 0.038 & 1 & 1.000 & 0.83 & 0.42 & 1.5 & 0.249 & 603 & 6.64 \\
\hline 11425 & -0.147 & 0.202 & 2 & 0.028 & & & & & & \\
\hline 11455 & -0.351 & 0.100 & 1 & 0.910 & & & & & & \\
\hline 11585 & -0.201 & 0.056 & 1 & 0.329 & & & & & & \\
\hline 11750 & -0.231 & 0.185 & 2 & 0.060 & & & & & & \\
\hline 11806 & -0.088 & 0.181 & 2 & 0.137 & & & & & & \\
\hline 11821 & -0.316 & 0.024 & 1 & 0.010 & & & & & & \\
\hline 11888 & -0.245 & 0.283 & 2 & 0.221 & & & & & & \\
\hline 11918 & -0.166 & 0.024 & 1 & 0.030 & & & & & & \\
\hline 11942 & -0.141 & 0.106 & 2 & 0.053 & & & & & & \\
\hline 12115 & -0.339 & 0.280 & 2 & 0.015 & & & & & & \\
\hline 12253 & -0.268 & 0.194 & 2 & 0.138 & & & & & & \\
\hline 12309 & -0.217 & 0.193 & 2 & 0.257 & & & & & & \\
\hline 12319 & -0.267 & 0.058 & 1 & 0.093 & & & & & & \\
\hline 12322 & -0.228 & 0.205 & 2 & 0.040 & & & & & & \\
\hline 12363 & -0.112 & 0.039 & 1 & 0.024 & & & & & & \\
\hline 12468 & -0.260 & 0.011 & 1 & 0.024 & & & & & & \\
\hline 12517 & -0.186 & 0.261 & 2 & 0.046 & & & & & & \\
\hline 12646 & -0.415 & 0.165 & 1 & 0.047 & & & & & & \\
\hline 12658 & -0.198 & 0.071 & 1 & 1.000 & 0.83 & 0.40 & 0.659 & 0.073 & 176 & 14.7 \\
\hline 12833 & -0.263 & 0.274 & 2 & 0.030 & & & & & & \\
\hline 13019 & -0.191 & 0.089 & 1 & 0.053 & & & & & & \\
\hline 13112 & -0.299 & 0.221 & 2 & 0.802 & & & & & & \\
\hline 13174 & -0.153 & 0.016 & 1 & 0.046 & & & & & & \\
\hline 13438 & 0.000 & 0.110 & 2 & 1.000 & 0.82 & 0.35 & 0.0676 & 0.022 & 5.93 & 123 \\
\hline 13521 & -0.195 & 0.059 & 1 & 0.013 & & & & & & \\
\hline 13556 & -0.310 & 0.080 & 1 & 0.045 & & & & & & \\
\hline 13739 & -0.417 & 0.279 & 2 & 0.090 & & & & & & \\
\hline 13768 & -0.073 & 0.029 & 1 & 0.012 & & & & & & \\
\hline 13808 & -0.334 & 0.319 & 2 & 0.695 & 0.83 & 0.21 & 4.13 & 0.112 & $3 e+03$ & 1.24 \\
\hline 13816 & -0.541 & 0.165 & 1 & 0.834 & 0.83 & 0.11 & 0.864 & 0.166 & 302 & 3.19 \\
\hline 13824 & -0.247 & 0.243 & 2 & 0.168 & & & & & & \\
\hline 14175 & -0.209 & 0.060 & 1 & 0.057 & & & & & & \\
\hline 14302 & -0.091 & 0.006 & 1 & 0.040 & & & & & & \\
\hline 14465 & -0.259 & 0.268 & 2 & 0.052 & & & & & & \\
\hline
\end{tabular}

Notes. For each star, we provide the ID in the photometric catalogue of Anderson et al. (2008), the location in the chromosome map, the population tag, the binary probability, the mass of the primary star, the minimum mass of the companion star, the semi-major axis, eccentricity, and period of the orbit, and its hardness. ${ }^{(a)}$ Assuming the orbit is orientated edge-on (i.e. $i=90 \mathrm{deg}$ ).

A65, page 5 of 6 
Chapter 2. Publications

A\&A 635, A65 (2020)

Table A.1. continued.

\begin{tabular}{|c|c|c|c|c|c|c|c|c|c|c|}
\hline ACS Id & $\Delta_{275,814}$ & $\Delta C_{275,336,438}$ & Pop. & $P_{\text {bin }}$ & $m_{\mathrm{p}} / M_{\odot}$ & $m_{\mathrm{c}} \sin i / M_{\odot}$ & $a / \mathrm{AU}^{(a)}$ & $e$ & $T / \mathrm{d}$ & $h$ \\
\hline 14601 & -0.331 & 0.071 & 1 & 0.047 & & & & & & \\
\hline 14789 & -0.059 & -0.003 & 1 & 0.075 & & & & & & \\
\hline 14815 & -0.248 & 0.250 & 2 & 0.030 & & & & & & \\
\hline 14830 & -0.404 & 0.358 & 2 & 0.022 & & & & & & \\
\hline 15012 & -0.035 & -0.002 & 1 & 0.074 & & & & & & \\
\hline 15013 & -0.280 & 0.272 & 2 & 0.104 & & & & & & \\
\hline 15069 & -0.233 & 0.274 & 2 & 0.016 & & & & & & \\
\hline 15101 & -0.212 & 0.203 & 2 & 0.047 & & & & & & \\
\hline 15165 & -0.157 & 0.202 & 2 & 0.007 & & & & & & \\
\hline 15182 & -0.144 & 0.014 & 1 & 0.018 & & & & & & \\
\hline 15293 & -0.536 & 0.158 & 1 & 1.000 & 0.82 & 0.49 & 1.64 & 0.477 & 669 & 7.16 \\
\hline 15382 & -0.146 & 0.023 & 1 & 0.579 & & & & & & \\
\hline 15422 & -0.143 & 0.234 & 2 & 0.041 & & & & & & \\
\hline 15482 & -0.284 & 0.077 & 1 & 1.000 & & & & & & \\
\hline 15528 & -0.300 & 0.267 & 2 & 0.052 & & & & & & \\
\hline 20774 & -0.270 & 0.176 & 2 & 0.042 & & & & & & \\
\hline 21050 & -0.202 & 0.211 & 2 & 0.167 & & & & & & \\
\hline 21060 & -0.084 & -0.009 & 1 & 0.129 & & & & & & \\
\hline 21131 & -0.039 & 0.036 & 1 & 0.115 & & & & & & \\
\hline 21189 & -0.158 & 0.178 & 2 & 0.005 & & & & & & \\
\hline 21232 & -0.142 & -0.006 & 1 & 1.000 & & & & & & \\
\hline 21271 & -0.027 & -0.012 & 1 & 0.051 & & & & & & \\
\hline 21272 & -0.252 & 0.296 & 2 & 0.039 & & & & & & \\
\hline 21273 & -0.282 & 0.221 & 2 & 0.072 & & & & & & \\
\hline 21292 & -0.113 & 0.183 & 2 & 0.047 & & & & & & \\
\hline 21707 & -0.249 & 0.240 & 2 & 0.011 & & & & & & \\
\hline 21918 & -0.275 & 0.264 & 2 & 0.008 & & & & & & \\
\hline 21921 & -0.101 & 0.021 & 1 & 0.999 & & & & & & \\
\hline 22325 & -0.099 & 0.166 & 2 & 0.042 & & & & & & \\
\hline 22396 & -0.011 & -0.008 & 1 & 0.022 & & & & & & \\
\hline 22401 & -0.130 & 0.093 & 2 & 0.163 & & & & & & \\
\hline 22488 & -0.317 & 0.262 & 2 & 0.320 & & & & & & \\
\hline 22686 & -0.376 & 0.252 & 2 & 0.029 & & & & & & \\
\hline 22751 & -0.556 & 0.120 & 1 & 1.000 & 0.83 & 0.30 & 0.631 & 0.027 & 173 & 11.4 \\
\hline 23045 & -0.255 & 0.218 & 2 & 0.051 & & & & & & \\
\hline 23175 & -0.200 & 0.022 & 1 & 1.000 & 0.83 & 0.12 & 0.491 & 0.124 & 129 & 5.78 \\
\hline 23330 & -0.030 & 0.001 & 1 & 0.010 & & & & & & \\
\hline 23342 & -0.101 & -0.005 & 1 & 0.465 & & & & & & \\
\hline 23375 & -0.232 & 0.246 & 2 & 0.487 & & & & & & \\
\hline 23452 & -0.257 & 0.241 & 2 & 1.000 & 0.82 & 0.20 & 0.69 & 0.056 & 206 & 7.01 \\
\hline 23461 & -0.181 & 0.265 & 2 & 0.040 & & & & & & \\
\hline 23519 & -0.212 & 0.034 & 1 & 0.046 & & & & & & \\
\hline 23640 & -0.198 & 0.155 & 2 & 0.044 & & & & & & \\
\hline 24190 & -0.140 & 0.271 & 2 & 0.007 & & & & & & \\
\hline 24416 & -0.276 & 0.253 & 2 & 0.012 & & & & & & \\
\hline 24524 & -0.098 & -0.003 & 1 & 0.220 & & & & & & \\
\hline 24592 & -0.151 & 0.033 & 1 & 0.045 & & & & & & \\
\hline 24594 & -0.355 & 0.352 & 2 & 0.024 & & & & & & \\
\hline 24684 & -0.272 & 0.400 & 2 & 0.028 & & & & & & \\
\hline 24753 & -0.007 & 0.005 & 1 & 0.030 & & & & & & \\
\hline 24803 & -0.149 & 0.037 & 1 & 0.031 & & & & & & \\
\hline 24832 & -0.266 & 0.258 & 2 & 0.040 & & & & & & \\
\hline 24875 & -0.231 & 0.269 & 2 & 0.030 & & & & & & \\
\hline 25058 & -0.185 & 0.041 & 1 & 0.037 & & & & & & \\
\hline 25322 & -0.317 & 0.076 & 1 & 0.799 & & & & & & \\
\hline
\end{tabular}

A65, page 6 of 6 


\section{Conclusions \& Outlook}

A significant fraction of the MUSE observing time was dedicated to multiple observations of the same FoVs within the globular cluster sample. From the beginning it was planned that these multi-epoch observations can be used to identify radial velocity variable stars which otherwise can affect other studies based on not time-resolved radial velocities. In addition, these radial velocities can be used to study the population of binary systems in a globular cluster. This thesis presents the most comprehensive study of binary stars in a globular cluster to date. It shows the first detection of a subsystem of stellar-mass black holes and puts crucial constraints on the formation channels of blue stragglers. The detailed comparison between the MUSE data and state-of-the-art Monte Carlo simulations results in strong constraints on the formation of NGC 3201.

\subsection{A new statistical method}

In this thesis a new statistical method was introduced to identify, in general, variations in sparse and noisy data of a large inhomogenous sample. It tests the null hypothesis that in all test subjects only statistical variations are present. In previous studies often a simple chi-square test was performed on individual objects and a star was classified to be in a binary system if the $3 \sigma$ probability was exceeded. Instead, the new statistical method uses the whole sample to assign a binary probability to each star. For example, $50 \%$ of all stars with the probability $50 \%$ are binaries, hence these individual stars are equally likely to be binaries and singles. For the globular cluster NGC 3201 the binary probability function is bimodal with many stars likely being single and some fraction likely being binary stars (see Fig. 6. in the second paper in Chapter 2). In between there are relatively few stars for which the result is not that clear, which demonstrates the strength of this approach.

A key ingredient for a robust statistical analysis is the correctness of the radial velocity uncertainties. A proper propagation from the MUSE detectors to the radial velocities is almost impossible. The reduction of MUSE data by the standard MUSE pipeline (Weilbacher et al., 2012 , 2014) removes cosmic rays and other detector artefacts, but covariances between pixels are neglected in the resampling process. In addition, the extraction of stellar spectra from the data cubes with the PSF-fitting technique (PAMPELMUSE, Kamann et al., 2013) and the final fit of the spectra with models from the PHOENIX library (SPEXXY, Husser et al., 2013) produce new systematic and statistical uncertainties. To ensure the quality of the radial velocity sample, many improvements were introduced into the extraction process and fitting of spectra. 
In addition, a lot of effort was put into the selection of the final radial velocity sample and the attainment of realistic uncertainties.

This new statistical method is a rewarding starting point for many further analyses. For example, Kamann et al. (2018b) used this statistical analysis on stars in all globular clusters to exclude radial velocity variables from the sample. The velocity dispersion is usually the standard deviation of average star velocities within a given population. Time-resolved radial velocities from binary systems could affect this dispersion strongly. The exclusion of binaries from a sample, or the usage of the real Keplerian system velocity of the binary systems instead, results in a cleaned velocity dispersion. This is also important to derive velocity dispersion profiles of globular clusters which are often used in the search for IMBHs.

\subsection{The binary fraction of NGC 3201}

Furthermore, this new statistical method can directly be evaluated to obtain the observational binary fraction. For NGC 3201 the resulting discovery binary fraction is $(17.1 \pm 1.9) \%$. This is the fraction of binaries detectable in the data obtained from the MUSE observations. This fraction is biased due to observational limitations such as binary system inclinations, weak radial velocity amplitudes, magnitude and thus $\mathrm{S} / \mathrm{N}$ limits, and the limitation to the MUSE FoVs. From a dedicated MOCCA simulation of NGC 3201 we know the discovery efficiency due to inclinations and weak radial velocities is $85 \%$. This means that the observational binary fraction in the MUSE data is actually $(20.08 \pm 0.22) \%$. But the magnitude and FoV limits still remain. Again with help of the MOCCA simulation this observational binary fraction can be translated into the total binary fraction of $(6.75 \pm 0.72) \%$ for the whole globular cluster NGC 3201 including double compact object systems, luminous stars with compact companions etc. The main effect for the total binary fraction being so much lower than the observational binary fraction is mass segregation of binaries towards the cluster centre. Not only the MOCCA model shows a clear mass segregation within NGC 3201, but the effect is also directly visible within the MUSE FoVs (Fig. 8. in the second paper in Chapter 2). NGC 3201 is probably the first globular cluster for which the binary fraction has been determined as completely as in this study.

\subsection{Orbital parameters of 95 binaries}

It turned out that for 95 stars within the NGC 3201 sample enough observations were obtained to individually constrain the Keplerian orbit using THE JOKER (Price-Whelan et al., 2017). This significant sample holds a number of peculiar objects which tell us a lot about the binary content of globular clusters. For the first time, I compiled a period-eccentricity plot of binaries in a globular cluster (Fig. 11. in the second paper in Chapter 2) which shows, that eccentric orbits are common in globular clusters and periods range from $0.3 \mathrm{~d}$ to $3000 \mathrm{~d}$ with most binaries having periods around $50 \mathrm{~d}$ in NGC 3201. The derived binary companion masses range from 
$0.03 \mathrm{M}_{\odot}$ to $7.7 \mathrm{M}_{\odot}$ with a median around $0.4 \mathrm{M}_{\odot}$ (see Fig. 12. in the second paper in Chapter 2). This also proves the access to low-mass stars and compact high-mass objects from MUSE radial velocity measurements.

\subsection{Blue straggler stars}

As introduced in Sect. 1.1.4, blue straggler stars could be important test-particles to probe the evolution history of globular clusters. Furthermore, the efficiency of the formation channels are highly debated. Sect. A.3.3 shows, that the binary fraction of blue stragglers is increased throughout all globular clusters within the MUSE globular cluster sample. For NGC 3201, an observational binary fraction of $(57.5 \pm 7.9) \%$ for the blue stragglers was found in this work. That is a surprising result, since a majority of blue stragglers, which are itself thought to be a product of binary evolution or stellar collision, are still in binary systems. The derived Keplerian orbits finally give conclusive evidence that two blue straggler formation channels are present in NGC 3201: mass transfer within short period blue straggler binary systems and the result of a coalescence of components in multi-star systems with more than two components. The latter one is most probably achieved by binary-binary encounters or within triple star systems. Thus a substantial contribution is made to the understanding of the formation of blue stragglers. In addition, the blue straggler binary properties are presented in detail and could be used to test globular cluster simulations.

Thanks to the matched literature data to the individual MUSE globular cluster stars (see Sect. A.4.5), five SX Phoenicis (SXP) stars have been identified within the blue straggler region in NGC 3201. The accepted theory is, these are pulsating blue straggler stars which have been formed in a binary system. A differentiation between radial velocities coming from radial pulsations and these induced by a companion in a binary system is currently not possible with sparse and noisy MUSE data. A future combination of the MUSE data with time-resolved photometry could help in this respect.

The binary system with the shortest period in the sample has previously been identified as a contact eclipsing binary with a period of $0.2975 \mathrm{~d}$ by Kaluzny et al. (2016, VN2). It is the best proof for a blue straggler in a binary system with ongoing mass transfer. The spectra show filled-in $\mathrm{H} \alpha$ absorption and the star is confirmed to be a cluster member based on the radial velocities. The period of $0.2697 \mathrm{~d}$ in the radial velocity data agrees with the literature and since the inclination is known, the companion mass of $(0.11 \pm 0.05) \mathrm{M}_{\odot}$ (assuming a blue straggler mass of $\left.(1.20 \pm 0.05) \mathrm{M}_{\odot}\right)$ is well determined (in this case changes in eccentricity and amplitude have only small impact on the companion mass). It looks like there is not much mass left of the donar star. Since the system is in contact, this remnant could coalesce with the blue straggler in the future. 


\subsection{Further peculiar binaries}

Further important insights are provided into the properties of four sub-subgiants (SSGs) or red stragglers (RSs), which have been identified by photometry, spectroscopy, and binary properties in the MUSE data. Two SSGs have known X-ray counterparts with one showing real $\mathrm{H} \alpha$ emission. The three other SSGs show filled-in H $\alpha$ absorption lines. All SSGs in the MUSE sample of NGC 3201 are binaries with binary properties agreeing with what is known from open clusters (Geller et al., 2017a). Probably this work describes these properties for the first time in a globular cluster. One SSG is a known eclipsing binary and allows to infer the actual companion mass of $(0.53 \pm 0.04) \mathrm{M}_{\odot}$. This is extremely interesting, since the probable host mass is $(0.82 \pm 0.05) \mathrm{M}_{\odot}$ and the binary system in total has a mass of $1.35 \mathrm{M}_{\odot}$. This mass would be enough to form a typical blue straggler, which is one of the possible evolution scenarios of SSGs (Geller et al., 2017b). Maybe the properties of this SSG could be used to exclude some of the other evolution scenarios in subsequent studies.

\subsection{Black holes}

In this work, two dynamically confirmed black holes with minimum masses of $(4.53 \pm 0.21) \mathrm{M}_{\odot}$ (BH1) and $(7.7 \pm 0.5) \mathrm{M}_{\odot}(\mathrm{BH} 2)$ were discovered in NGC 3201. Another candidate with a minimum mass of $(4.4 \pm 2.8) \mathrm{M}_{\odot}$ (BH3), which has yet to be confirmed, has been found in the same cluster as well. Given the MUSE radial velocities and HST photometry, these binary systems are certain members of NGC 3201. The anyway unlikely scenario of a double neutron star component as the binary companion for the visible component of the BH1 system can be completely excluded for the $\mathrm{BH} 2$ system, since the $\mathrm{BH}$ mass is simply too large. In contrast to the spectra of the quiescent $\mathrm{BH} 1$ companion, the spectra of the $\mathrm{BH} 2$ companion show a filled-in $\mathrm{H} \alpha$ absorption line which fits the short period of $(2.2422 \pm 0.0001) \mathrm{d}$. Thus the visible main-sequence star could be influenced by the $\mathrm{BH} 2$, but to date no radio or X-ray source is known in this cluster at that position. By the way, the Schwarzschild radius as of Eq. 1.2 is of the order of $10 \mathrm{~km}$ for the BHs discovered in this work.

Upon the first blind discovery of the detached black hole BH1 (see the first paper in Chapter 2) and an accompanying press release ${ }^{1}$, there was a strong response on the theoretical side. On the one hand, black holes in globular clusters are of great interest for predicting the gravitational wave signals measurable by gravitational wave detectors (today and in the future, e.g. Kremer et al., 2018a, Banerjee, 2018). On the other hand, especially the properties of black holes in binary systems (mass estimates, system configuration and so on) help a lot to constrain the retention fraction of black holes in globular clusters and thus the modelling of globular clusters (e.g. Kremer et al., 2018b, Askar et al., 2018, Arca Sedda et al., 2018, Kremer et al., 2019, Askar

${ }^{1}$ Odd Behaviour of Star Reveals Lonely Black Hole Hiding in Giant Star Cluster, ESO science press release No. eso1802 https://www.eso.org/public/news/eso1802/ 


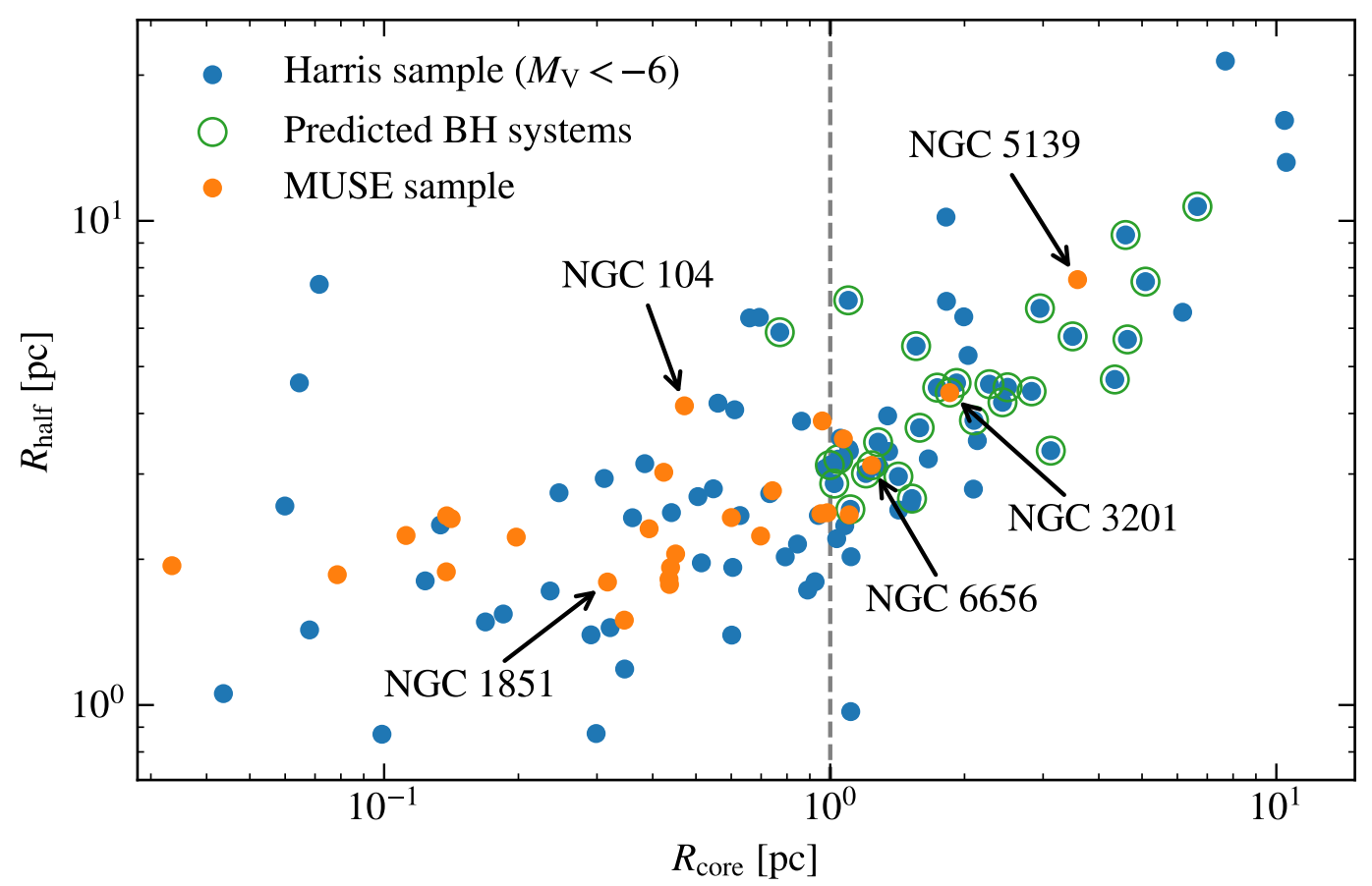

Figure 3.1.: Half-light radius of the globular clusters in the Harris (1996, 2010 edition) catalogue as a function of their core radius, with a limit on the absolute visual magnitude $M_{\mathrm{V}}<-6$. In orange, the MUSE sample is highlighted. Globular clusters with predicted BH systems by Askar et al. (2018) are indicated with green circles.

et al., 2019). In addition, it also drives other researchers to use similar observational methods to find such black hole systems, like with Gaia (e.g. Yalinewich et al., 2018), the APOGEE survey (Thompson et al., 2019), TESS (Masuda \& Hotokezaka, 2019), or the LAMOST survey (Gu et al., 2019).

\subsubsection{Retention fraction}

Only few years ago, theorists were convinced that no BHs (or only one) should stay in cluster cores. This work clearly proves that this can not be true. However, from last discussions about simulations with ASKAR and KREMER a conclusion was drawn that it is hard to gauge the total black hole population of a globular cluster only based on the in this work detected black holes. The number of black holes in a binary system with a luminous component appears (to a certain extent) to be independent of the total number of black holes. But if, in addition, other cluster properties like the binary fraction are considered, the retention fraction could be better constrained. In this work it was shown, taking the binary fraction into account, that the best fitting MOCCA model contains $43 \mathrm{BHs}$ which is lower than the suggested numbers of 
100 up to 200 BHs by Kremer et al. (2018b) and Askar et al. (2018). It was also shown that from different MOCCA models only the ones with high primordial binary fractions match the MUSE observations. This supports the hypothesis that high primordial binary fractions are necessary to reproduce the observations (see Sect. 1.1.3). At least for NGC 3201, future simulations should be judged by how well they agree with these observed facts.

Figure 3.1 shows the half-light radius of the globular clusters in the Harris (1996, 2010 edition) catalogue as a function of their core radius, with a limit on the absolute visual magnitude $M_{\mathrm{V}}<-6$. In orange, the MUSE sample is highlighted. Globular clusters with predicted BH systems by Askar et al. (2018) are indicated with green circles. Conclusively, three globular clusters from the MUSE sample are likely to harbour BH systems: NGC 3201, NGC 6656 and $\omega$ Cen $^{2}$. As previously mentioned, this work shows that NGC 3201 hosts at least two BHs and should have an extended black hole subsystem. The globular cluster $\omega$ Cen is currently under investigation, but preliminary analyses found no BH. First attempts of THE JOKER on $\omega$ Cen resulted in only a few well-defined binary systems with short periods around $1 \mathrm{~d}$. Since these were mostly discovered in the overlapping regions of the MUSE pointings, it seems that further observations with a time sampling in the hour range are needed (i.e. three observations per night and pointing).

\subsubsection{The search for an IMBH}

A combination of proper motions (PMs) and MUSE radial velocities would increase the sensitivity to find IMBHs in globular clusters. Even BHs with masses down to $50 \mathrm{M}_{\odot}$ could be discovered using only precise velocity dispersion profiles or multiple long baseline velocity measurements of single stars. For example, the sphere of gravitational influence of a $78 \mathrm{M}_{\odot} \mathrm{BH}$ - which is the most massive BH in the MOCCA model for NGC 3201 used in this work - has a radius of the order of $0.013 \mathrm{pc}\left(\approx 0.5^{\prime \prime}\right.$ in the distance of NGC 3201) using the definition of Peebles (1972) and the core velocity dispersion of $5 \mathrm{~km} \mathrm{~s}^{-1}$ in NGC 3201. The MUSE narrow field mode (NFM) with a FoV of $7.5^{\prime \prime} \times 7.5^{\prime \prime}$ and a spatial sampling of $0.025^{\prime \prime}$ is perfect for this purpose. Some clusters within the MUSE globular cluster sample are going to be observed with this NFM. For instance, $\omega$ Cen is a promising candidate to host an IMBH, for which, assuming a $1000 \mathrm{M}_{\odot} \mathrm{BH}$ and a stellar velocity dispersion of $17 \mathrm{~km} \mathrm{~s}^{-1}$, the sphere of influence would be of the similar order $\left(0.015 \mathrm{pc} \approx 0.6^{\prime \prime}\right)$ as a stellar-mass BH in NGC 3201 .

\subsection{Binaries in multiple populations}

The rich data set of NGC 3201 allowed us to extend the studies towards multiple populations. I used the "chromosome map" method on the red giant branch to divide the stars into two populations. Interestingly, a significant difference in the binary fractions appeared: I found the

${ }^{2}$ Globular cluster simulations are currently not able to reproduce $\omega$ Cen, that is why it is not included in Askar et al. (2018). 
observed binary fraction in the population P1 to be $(23.1 \pm 6.2) \%$ in contrast to $(8.2 \pm 3.5) \%$ in P2. This is in agreement with previous studies mentioned in Sect. 1.1.6, but since it focuses on the core of NGC 3201, the explanation, that the different fractions originate from different initial concentrations, is no longer valid. The signatures of different initial concentrations should vanish in the core of NGC 3201 due to its short relaxation time. Furthermore, we demonstrate that most, if not all, binaries in NGC 3201 are primordial and only destruction processes reduce the binary fractions. Hence, it is more plausible that the populations were formed with different primordial binary fractions. One conceivable scenario would be the formation of one homogeneous globular cluster population at first (P1) and at a later stage a formation of P2 stars within the P1 population. The presence of P1 stars could not allow to form that much primordial binary stars in the population P2.

The multiple population study also demonstrates that binary stars behave differently in the photometric "chromosome map". On the one hand, the two stars in the binary system contribute both to the flux measurements in the multiple filters. Of course, the measurement of RGB binary stars is dominated by the RGB star itself, but a contribution of the faint bluer component to the blue filters is possible and could alter the inherent position of the RGB in the "chromosome map". The assignment by this method for binary systems should therefore be treated with caution. On the other hand, we find the majority of stars on the blue extension of the population P1 in the "chromosome map" to be in binary systems. Theories are currently struggling to explain these stars and we would now like to point out that this apparently may have something to do with the binary evolution of these systems.

\subsection{Further outlook}

Once the observational sampling of 47 Tuc and $\omega$ Cen is sufficient (e.g. for short period binaries) they could be analysed in the same manner as shown in this work on the example of NGC 3201. Of course, sophisticated models, such as the MOCCA model for NGC 3201, would be desirable for these globular clusters as well. Maybe NGC 1851, which already has a median of seven observations per pointing (see Table 1.2), could be interesting, because it shows multiple complex populations with larger difference in metallicity than normal globular clusters have (see Sect. 1.1.6). As mentioned before, the globular cluster NGC 6656 (M22) could also host a black hole system and a binary study of this cluster could be promising. (M22 currently has only a median of 2 observations per pointing.) All three clusters appear to have small binary fractions $<5 \%$ (Milone et al., 2012).

Furthermore, it would be nice to determine the primordial binary fractions and to constrain the number of black holes in most Galactic globular clusters. This could also answer the question how many gravitational wave events originate from $\mathrm{BH}-\mathrm{BH}$ mergers in globular clusters. Moreover, a better understanding of the binary evolution could be achieved by studying younger massive clusters (e.g. in the LMC or SMC). 
In this thesis, a classification of the binary companions (e.g. stellar type) was in most cases not possible. On the one hand, the unknown inclination only allowed us to derive the minimum mass of the companions. On the other hand, it would be nice to isolate contributions of the companion in the observed spectrum or to use precise photometric measurements to get clues on the spectral type of the companion. Thus, observations by the upcoming Extremely Large Telescope (ELT) or James Webb Space Telescope (JWST) should be able, for example, to better constrain the remnant fraction of compact or degenerated objects in globular clusters.

In Kamann et al. (2018a) it has been shown that from stellar spectra - obtained with MUSE it is also possible to determine the stellar rotation (hereafter called spin velocity) of individual stars. The detection limit is approximately at a spin velocity of $V \sin i \approx 30 \mathrm{~km} \mathrm{~s}^{-1}$ within a spectrum of $S / N=20$. Spin velocities within the old populations of the MUSE globular cluster sample should be in general below this detection limit (Skumanich, 1972), which is why it was only investigated in young clusters like in the mentioned publication. However, blue straggler stars are rejuvenated stars which indeed could have spin velocities significantly larger than $30 \mathrm{~km} \mathrm{~s}^{-1}$. A study in NGC 3201 by Simunovic \& Puzia (2014) found several fast rotating blue stragglers which are in common with the MUSE sample of NGC 3201. Starting from slowly rotating stars, a blue straggler could gain angular momentum either from mass transfer of the donar star or from the collision (coalescence) of two stars, but currently convincing theoretical studies are missing to give a useful interpretation in respect to the formation channel on the spin velocities only. Nevertheless, applied to all blue straggler stars within the MUSE globular cluster sample and combined with orbital analyses, the spin velocities could contribute to a deeper understanding of the different formation circumstances of blue stragglers. A description how to get the spin velocities for individual MUSE spectra is given in Sect. A.5.

Another interesting aspect could be the study of the pairing function of the binary orbital parameters in globular clusters, like the 95 binaries in NGC 3201 have. As explained in the second paper in Chapter 2, the assumption was made that the probability density function of the orbital parameters $f\left(M_{1}, M_{2}, P, e\right) \neq f\left(M_{1}\right) f\left(M_{2}\right) f(P) f(e)$ can not be simply reconstructed from the individual probability density functions. If this assumption does not apply to globular clusters, it might mean that the binary content could be modelled more easily than by complete cluster simulations.

All in all, this pioneering work could be seen as a reference study for future spectroscopic binary studies. It presents necessary methods and tools to do complete binary studies in upcoming big data projects. 


\section{A. Appendix}

In this appendix, I will give more detailed information on the work carried out in the publications and what else was done during my PhD project. Section A.1 describes the differential photometry method to identify photometrically varying stars based on MUSE observations. In Section A.2 an early approach for the simulation of radial velocities of a realistic globular cluster is discussed. In Section A.3 preliminary results of an interim method applied to all clusters are presented: The model based expectation maximisation (EM) method to identify binary stars in MUSE globular cluster observations is explained in Sect. A.3.1. The behaviour and robustness of this method is then checked on cluster simulations in Sect. A.3.2. Consequently, it is applied to the MUSE globular cluster sample and the resulting binary fractions of the globular clusters are compared with each other in Sect. A.3.3.

Important for this big data project was the use of several literature catalogues in conjunction with the MUSE data described in Sect. A.4. The combination of many different sources with our observational data provides new insights as shown in the second paper in Chapter 2. Lately, we found it is also possible to study stellar rotation with MUSE spectra. Instructions for that are given in Sect. A.5. As a by-product of this PhD project, a systematic way to normalise spectra was found, which is summarised in Sect. A.6.

\section{A.1. Differential photometry}

In order to identify photometric variable stars in our MUSE sample, we usually use the Catalogue of Variable Stars in Galactic Globular Clusters (Clement et al., 2001, Clement, 2017). This catalogue is conducted from a multitude of publications with different objectives and is therefore not complete. The crowded cores of globular clusters could harbour some pulsating stars, chromospheric active stars, or eclipsing binaries that might still be undetected. Stars in the instability strip, like RR Lyrae-type stars or SX Phoenicis-type stars, are known to exist in globular clusters, and those stars with radially pulsating modes can show radial velocity amplitudes up to $50 \mathrm{~km} \mathrm{~s}^{-1}$. We should be aware of these stars because a false detection of a pulsating star as a binary star would influence the overall statistics and further analyses of this star. For that reason, we implemented a method, which could find photometric variables directly using the multi-epoch observations done with MUSE. In principle, the flux of individual spectra could be measured and compared with each other. Although the MUSE instrument and pipeline ensures that flux levels are calibrated over different observations, the reconstruction of magnitudes just from the extracted spectra may still contain systematic errors. For example, since our targets 


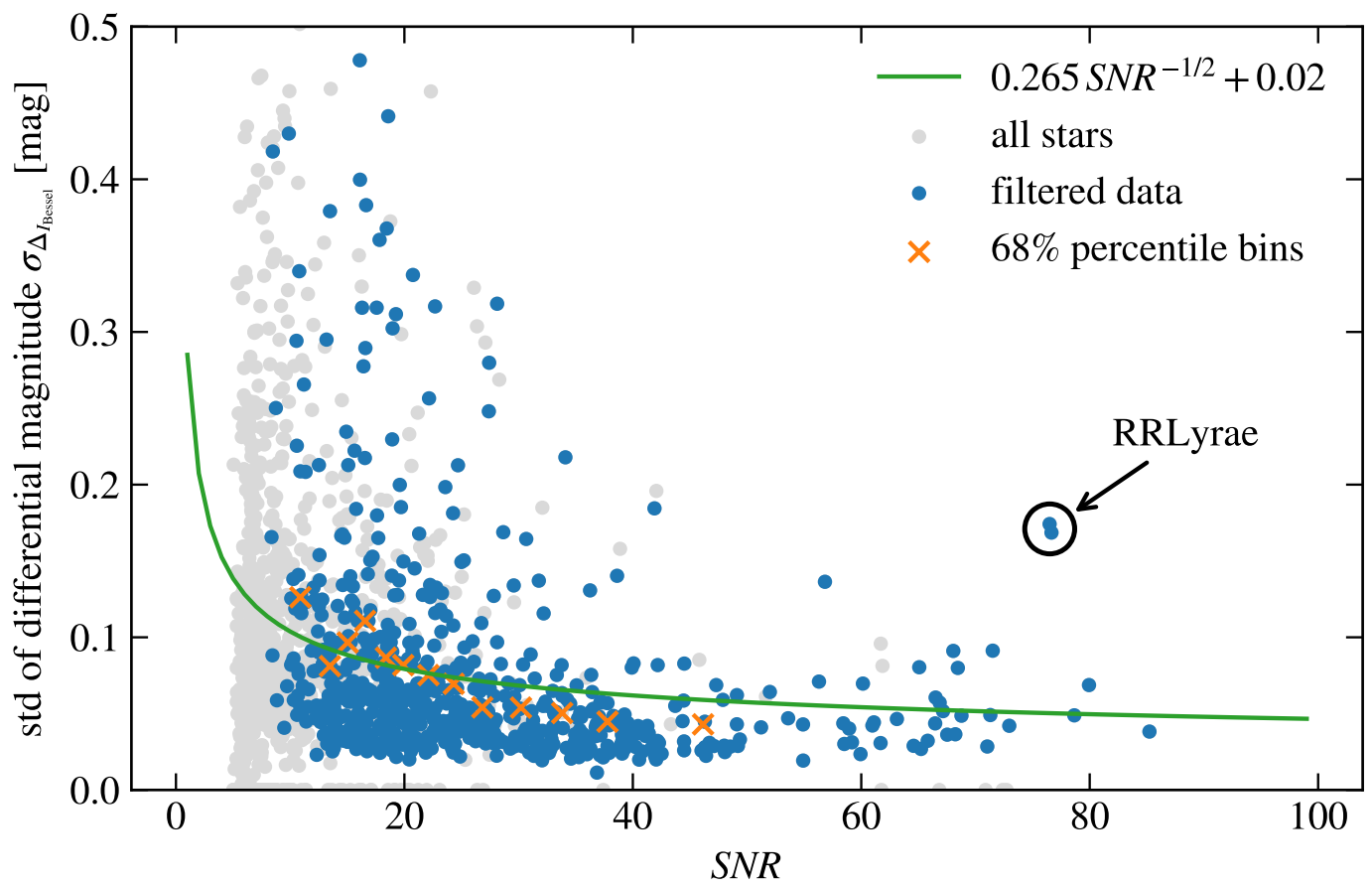

Figure A.1.: The standard deviation of the differential magnitudes of all epochs and stars in pointing 01 of the MUSE data in NGC 3201. Blue points show the MUSE data with each star having 13 observations. Orange markers represent the $68 \%$ percentile in SNR bins with 50 stars per bin. The green line shows an approximation of the data with a simple square root function. The slope of this function was fitted to the data.

are relatively bright, our observations are also done during grey time or twilight. To save observation time we do not observe separate sky exposures and only model the sky component afterwards. This is only one possible contribution to a stellar spectrum. But relative fluxes of sources within the same FoV in one observation should be unaffected by most systematic effects. That is why we use the differential photometry approach to find variable stars.

The classical differential photometry technique uses one target star and one or several reference stars to compare with in the same FoV (e.g. Kern \& Bookmyer, 1986). Of course, the reference star should be stable over time and have preferentially similar magnitude (and colour) as the target star in the chosen filter to avoid other systematic effects. Then, the difference magnitude between target and reference star over time is simply the relative brightness of the target star compared to the reference star. For the MUSE globular cluster observations no suitable reference stars are known in the first place. We also have the crowding problem which means that several star PSFs could interfere with each other. Fortunately, the crowding problem is in most cases solved with the PSF-fitting technique developed by Kamann et al. (2013). This technique works best, when the positions of the PSFs in the MUSE observation are pre- 


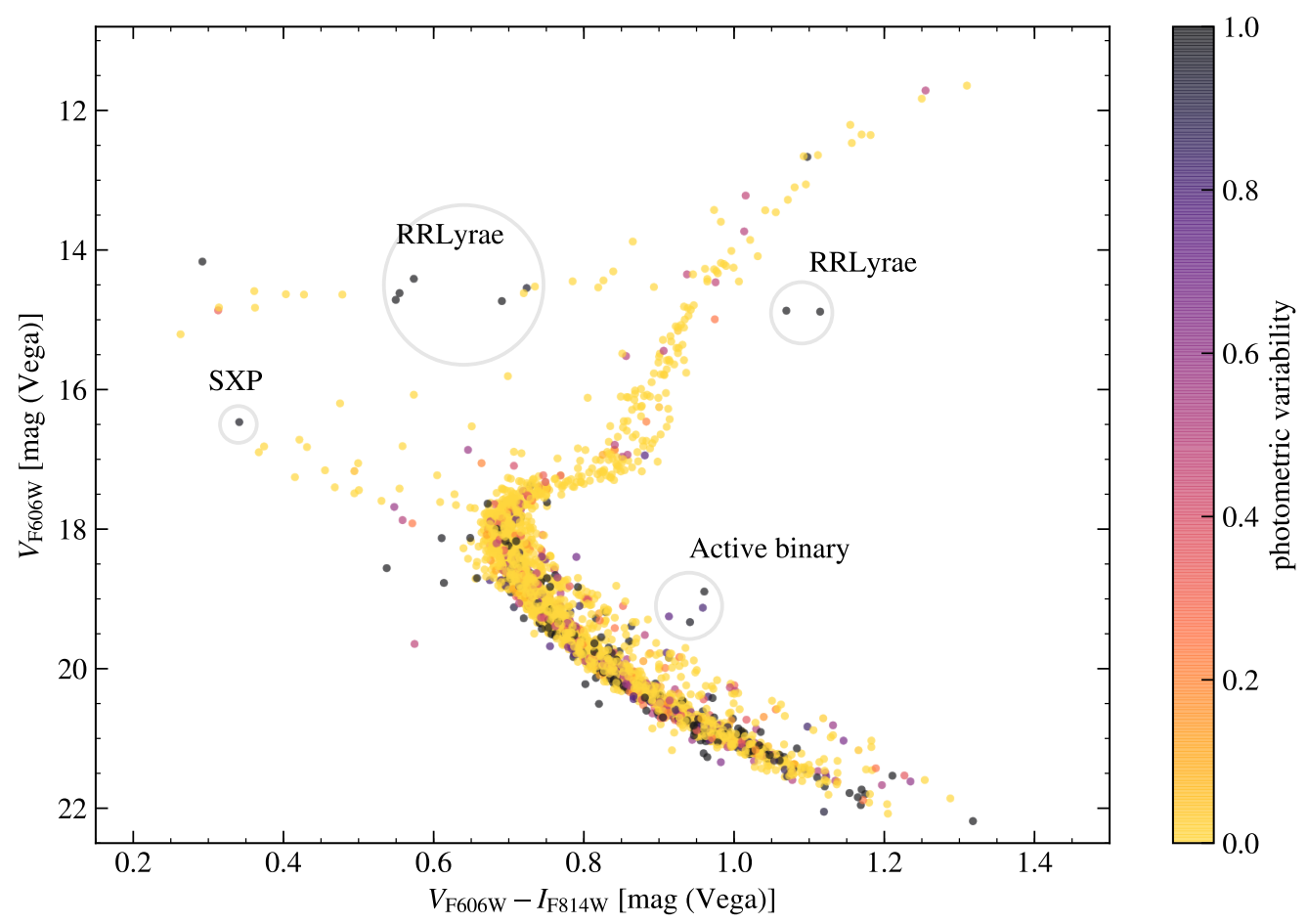

Figure A.2.: CMD of NGC 3201 with the colour-coded photometric variability determined from MUSE spectra using the statistical method of (see the second paper in Chapter 2). Known photometric variable star types are highlighted in the figure.

cisely known, with not too much residuals of other spectra, and the signal-to-noise (SNR) in the sources is not too low (Kamann et al., 2016). Under the assumption that the PSF of a star is well fitted, the extracted spectrum of that source should be free from contamination. For all extracted spectra of all observations, magnitudes of different photometric filters (e.g. Bessel system, HST instrument filters) are calculated.

To find appropriate reference stars, an iterative approach is chosen. Reference stars should be present in all observations of the same FoV, have good SNR, and show no photometric variations (intrinsically or due to crowding). Up to a hundred reference stars with an intrinsic standard deviation of $0.005 \mathrm{mag}$ in the filter $I_{\text {Bessel }}$ typically fulfil these conditions in one FoV. Then, we compare the magnitudes of every star from each observation with our reference stars, selecting 20 with comparable colour. Finally, the uncertainty of each measurement is calculated from the distribution of all measurements per observation depending on SNR. The slope of a simple square root function is fitted to the standard deviation of $68 \%$ percentile SNR bins, assuming a minimal uncertainty of $0.02 \mathrm{mag}$ (y-intercept). In Fig. A.1 the result of this method for one example pointing of NGC 3201 is shown. The median differential magnitude accuracy is $0.066 \mathrm{mag}$. This is not exceptional, but sufficient for the identification of, for example, RR 
Lyrae stars. In NGC 3201 almost all known RR Lyrae stars show variable photometry and also enables the identification of unknown variable stars with similar properties.

Fig. A.2 shows the photometric variable stars of all MUSE NGC 3201 pointings in a CMD. The probability per star was calculated using the statistical method introduced in the second paper in Chapter 2. The RR Lyrae stars, SXP stars, and some chromospheric active binaries show clear photometric variations. The RR Lyrae stars and SXP stars are already known in the literature, but for some of them the confirmation was missing so that they can be included in the Clement (2017) catalogue. The majority of varying stars at the main sequence are probably false detections: These could be caused by an inaccurate spectrum extraction and thus false magnitude measurement due to blends of other sources.

Another approach to find variable stars in the MUSE data, without relying on the PSF extraction, would be difference image photometry. With the creation of 2D images (collapse of some wavelength region) from the MUSE observations, the difference image of two independent observations could be calculated. Ideally, for this technique the observational conditions (e.g. seeing, airmass) should be the same. Then, only varying stars should be left in the difference images.

\section{A.2. Simulation: Globular cluster model of radial velocities}

In the following a toy model for obtaining a set of radial velocity measurements from a cluster containing single and binary stars is introduced. A basic assumption of this model is that due to tidal interactions in binaries the orbits become circular over many periods (Hut et al., 1992). In the second paper in Chapter 2 it was shown that this assumption is wrong. Nevertheless, this model and its application to test methods are presented here. ${ }^{1}$

All stars in a globular cluster are considered to be isolated systems. Radial velocities of single stars are constant, whereas the radial velocities from binaries in circular orbits can be calculated with Eq. 1.7 from the introduction.

The mass of the primary (observed) star is taken from an isochrone fitted to the HST data of each globular cluster (see the second paper in Chapter 2 for details). For the initial companion mass $M_{\text {ini. }}$ an altered Salpeter (1955) IMF is used, which is flat below one solar mass (Miller \& Scalo, 1979) and starts at the minimum mass of brown dwarfs at 0.013 solar masses:

$$
\operatorname{IMF}\left(M_{\text {ini. }}\right) \propto \begin{cases}\text { const. } & \text { if } 0.013 \mathrm{M}_{\odot}<M_{\text {ini. }} \leq 1.0 \mathrm{M}_{\odot} \\ M_{\text {ini. }}^{-2.35} & \text { if } 1.0 \mathrm{M}_{\odot}<M_{\text {ini. }} \leq 8.0 \mathrm{M}_{\odot} \\ 0 & \text { else } .\end{cases}
$$

Masses of individual stars change over the lifetime of a globular cluster. Especially stars with masses higher than the turnoff mass $M_{\text {turnoff }}$ evolve. The initial mass $M_{\text {ini. }}$ of the companion has

\footnotetext{
${ }^{1}$ In a later version of this toy model, eccentricity was introduced with the use of Eq. 1.12.
} 


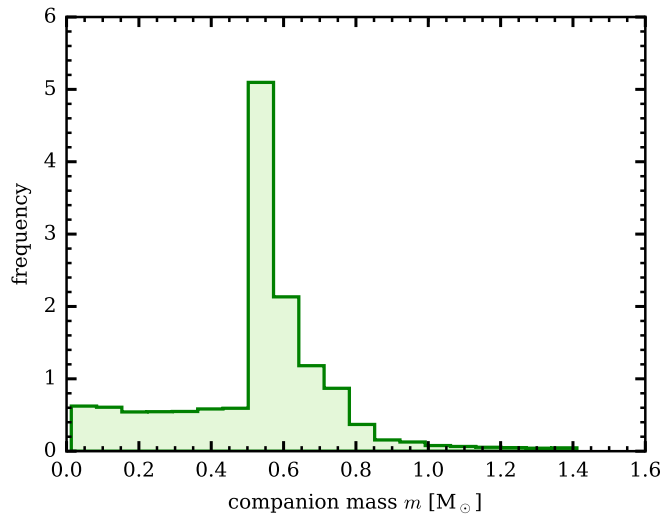

(a) The simulated companion mass distribution of binary systems in a globular cluster with a turnoff mass of $M_{\text {turnoff }}=0.8 \mathrm{M}_{\odot}$.

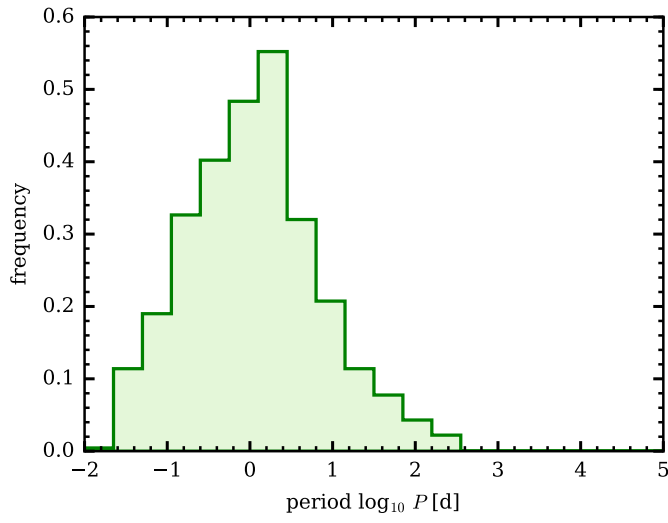

(b) The simulated period distribution of binaries in a globular cluster with the density $\log \rho_{\mathrm{M}}=5.7$ (e.g. NGC 6388).

Figure A.3.: Simulated companion mass and period distributions of binaries in a globular cluster.

to be converted into an actual mass $M_{\text {act. }}$ which can be done following Catalán et al. (2008):

$$
M_{\text {act. }}= \begin{cases}0.096(5) M_{\text {ini. }}+0.429(15) & \text { if } M_{\text {turnoff }}<M_{\text {ini. }} \leq 2.7 \mathrm{M}_{\odot} \\ 0.137(7) M_{\text {ini. }}+0.318(18) & \text { if } M_{\text {ini. }}>2.7 \mathrm{M}_{\odot} .\end{cases}
$$

These equations model the loss of mass during stellar evolution. Some stars have already become white dwarfs or neutron stars. An example result of the simulated companion mass distribution of the globular cluster NGC 6388 is shown in Fig. A.3a. Most of the companions are found to have a mass around $0.5 \mathrm{M}_{\odot}$ which is in agreement with the average star mass in an old Galactic globular cluster. A large portion of this peak could be attributed to white dwarfs.

Finally, we need the period distribution for the model to get realistic radial velocities. To avoid making to many assumptions on this, we use the period distributions as a function of the globular cluster density found in n-body simulations by Ivanova et al. (2005). The globular cluster densities are taken from the catalogue Pryor \& Meylan (1993). Figure A.3b shows the simulated period distribution of the globular cluster NGC 6388 with a density of $\log \rho_{\mathrm{M}}=5.7$.

For the simulation of binary stars we use a random selection from the primary mass, companion mass, uniform distributed inclination, and period distribution as described before. We make sure, that the companion star is not brighter than the primary star and that the system is physically possible (binary separation larger than Roche lobe and not too large in respect to cluster velocity dispersion and cluster density, see Hut et al. (1992)). 


\section{A.3. Binary fractions in the MUSE globular cluster sample}

\section{A.3.1. Expectation maximisation technique to identify binary stars}

To detect radial velocity variations in a single star, we use a $\chi^{2}$-statistic to measure how compatible the radial velocities over time are with the constant weighted mean $\bar{x}$ (null hypothesis) of the $n$ radial velocity measurements $x_{i}$ with uncertainty $\sigma_{i}$ :

$$
\chi^{2}=\sum_{i=1}^{n} \frac{1}{\sigma_{i}^{2}}\left(x_{i}-\bar{x}\right)^{2} \quad \text { with } \quad \bar{x}=\frac{\sum_{i=1}^{n} \frac{x_{i}}{\sigma_{i}^{2}}}{\sum_{i=1}^{n} \frac{1}{\sigma_{i}^{2}}} .
$$

As described in the second paper in Chapter 2 each star in our sample has its own number of observations and therefore its own number of degrees of freedom $\nu$. Assuming Gaussian distributed uncertainties we know the probability density function (PDF) expected for a specific $\nu$ if the null hypothesis for all stars is true. If this is not the case we have variable stars in our sample. To find the fraction of binary stars $f_{\text {binaries }}$ in our sample and to estimate the probability of each star individually we use an expectation maximisation (EM) analysis. This analysis finds the optimal contribution of two known independent populations in a given sample. Following Walker et al. (2009, equations 3 and 4) we came up with the expectation step

$$
P_{\text {single }}\left(\chi^{2}, \nu\right)=\frac{p_{\text {single }}\left(\chi^{2}, \nu\right)\left[1-f_{\text {binaries }}\right]}{p_{\text {single }}\left(\chi^{2}, \nu\right)\left[1-f_{\text {binaries }}\right]+p_{\text {binary }}\left(\chi^{2}, \nu\right) f_{\text {binaries }}}
$$

with $P_{\text {single }}$ for the probability of each star to be single and the maximisation step

$$
f_{\text {binaries }}=\left[\frac{\sum_{j=1}^{N} P_{\text {single }}\left(\chi_{j}^{2}, \nu_{j}\right)}{\sum_{j=1}^{N}\left(1-P_{\text {single }}\left(\chi_{j}^{2}, \nu_{j}\right)\right)}+1\right]^{-1}
$$

with the binary fraction $f_{\text {binaries. }}$ In Eq. A.4, the probability $p_{\text {single }}$ to belong to the single star distribution is

$$
p_{\text {single }}\left(\chi^{2}, \nu\right)=\operatorname{PDF}_{\text {single }}\left(\chi^{2}, \nu\right) \text {. }
$$

Consequently, the probability to belong to the binary star distribution is

$$
p_{\text {binary }}\left(\chi^{2}, \nu\right)=\operatorname{PDF}_{\text {binary }}\left(\chi^{2}, \nu\right)
$$

With these equations, the best solution for the binary fraction $f_{\text {binaries }}$ and the resulting single star probability $P_{\text {single }}$ of each star can be found iteratively. The binary star probability of each star is modest

$$
P_{\text {binary }}\left(\chi^{2}, \nu\right)=1-P_{\text {single }}\left(\chi^{2}, \nu\right) \text {. }
$$




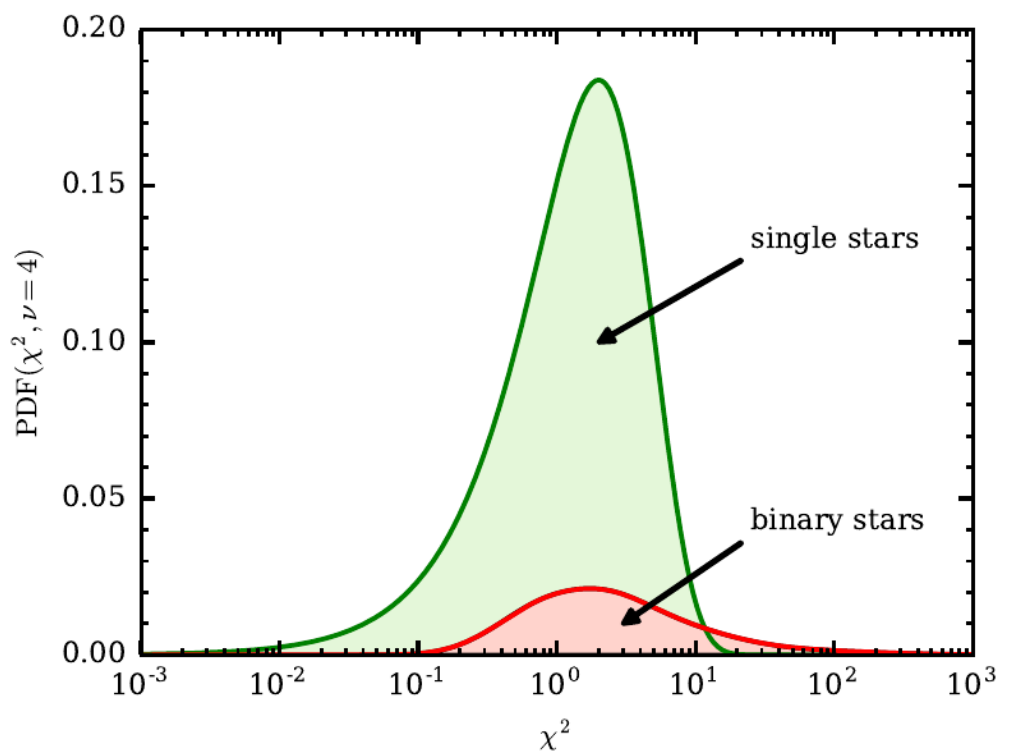

Figure A.4.: The simulated probability density function (PDF) of the radial velocity $\chi^{2}$ distribution of a single and binary star population. The simulation of the binary PDF was realised with data from NGC 6388 and exactly 5 epochs $(\nu=4)$ for each of 1000 binary stars.

We know the expected $\chi^{2}$ distribution of single stars (simply from the Gaussian PDF) and we know the expected $\chi^{2}$ distribution of binary stars from our simulations described in Sect. A.2. To get the PDF of the binary stars we use a kernel density estimation (KDE) using Gaussian kernels to reconstruct the PDF in log-space and transform the resulting KDE with

$$
\operatorname{PDF}_{\text {linear-space }}\left(\chi^{2}, \nu\right)=\frac{\operatorname{PDF}_{\text {log-space }}\left(\log \left(\chi^{2}\right), \nu\right)}{\chi^{2}}
$$

back to linear-space. An example for the resulting PDFs for the single star and binary star population of a globular cluster is shown in Fig. A.4. The integral of both functions is, of course, one in linear space.

\section{A.3.2. Sanity checks of expectation maximisation on simulations}

To get confidence in the EM method and to get an idea of how the method reacts to systematic effects, we have done many simulations and their analyses. The first check is, of course, the coherence of the resulting binary fraction with the simulated one. The results for different simulated binary fractions are shown in Fig. A.5a. The plot shows that the analysis yields always the simulated binary fraction. Our next check was to exclude systematics on the number of epochs $N_{\text {epochs. }}$. Figure A.5b shows, for a fixed binary fraction, that there is no effect on $N_{\text {epochs }}$.

We conclude that the method is robust if the simulated and analysed model are the same. 


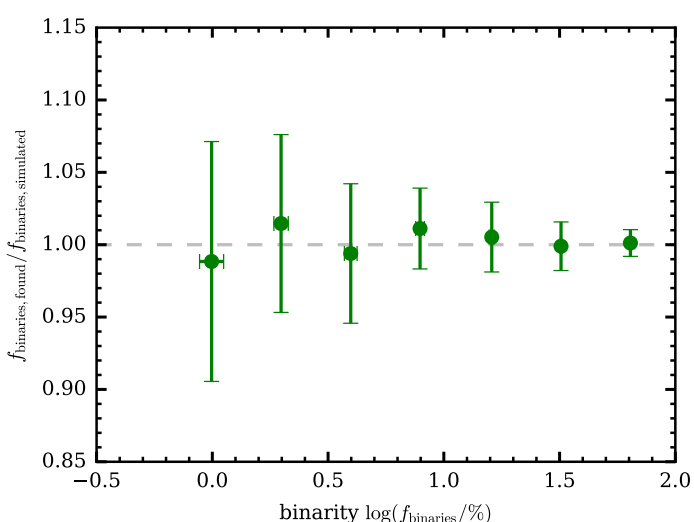

(a) Using different binary fractions. The fraction of analysed and simulated binaries is plotted as a function of the simulated binary fraction. 30 simulations per point for statistics.

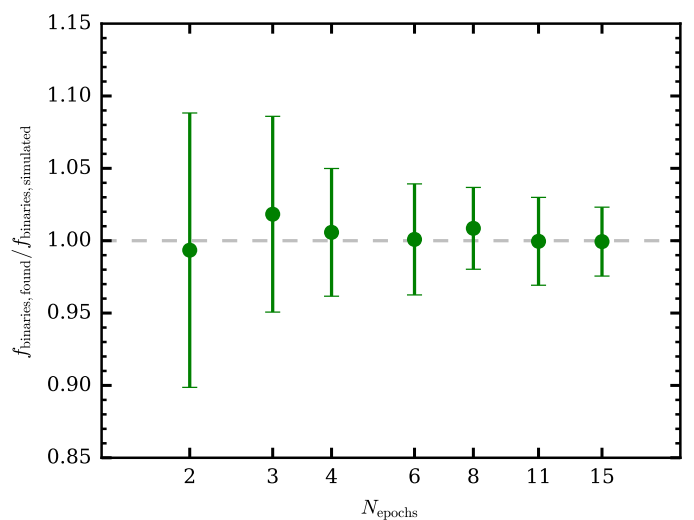

(b) Using different number of epochs and a fixed binary fraction $f_{\text {binaries }}=10 \%$. The fraction of analysed and simulated binaries is plotted as a function of the unique epoch. 100 simulations per point for statistics.

Figure A.5.: The expectation maximisation method applied to different simulations showing robust behaviour.

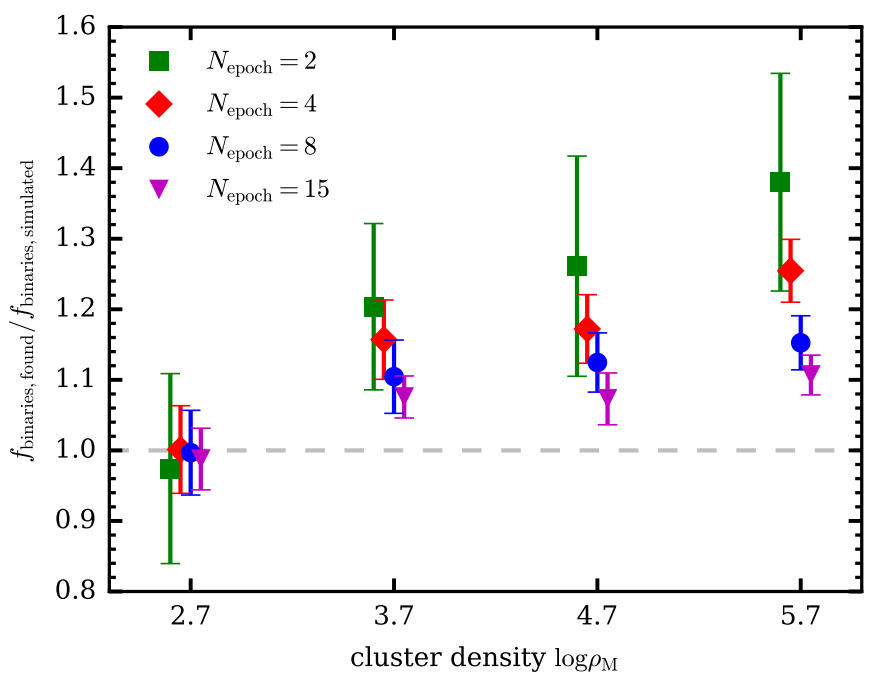

Figure A.6.: The expectation maximisation method applied to simulations with different epochs and different cluster densities. The binary fraction $f_{\text {binaries }}=10 \%$ and the analysis cluster density $\log \rho_{\mathrm{M}}=2.7$ are fixed. The fraction of analysed and simulated binaries for different unique epochs is plotted as a function of the simulated cluster density. 100 simulations per point for statistics. For better visibility the results are slightly shifted on the $\mathrm{x}$-axis. 


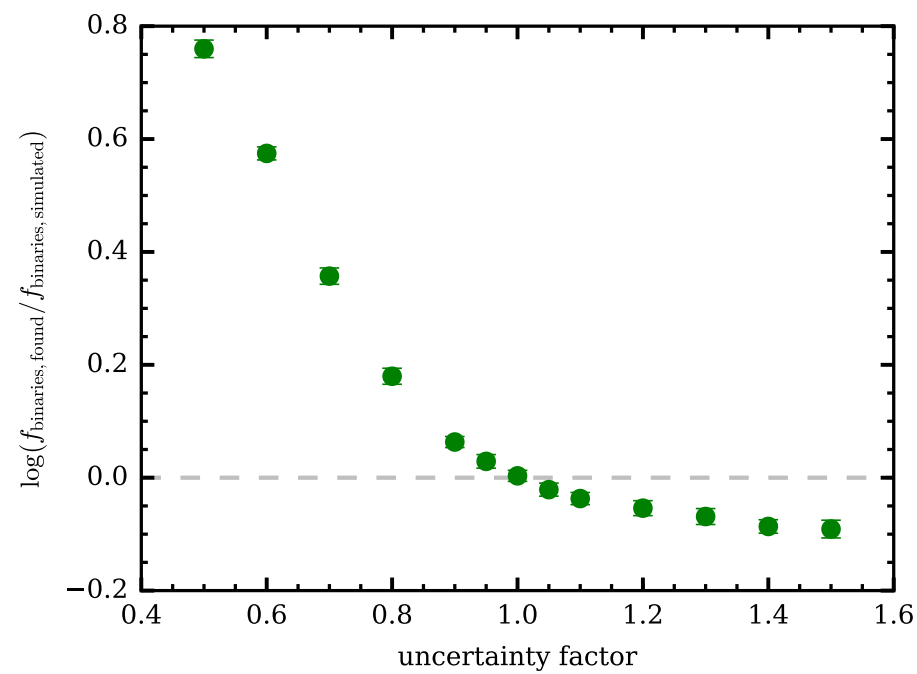

Figure A.7.: The expectation maximisation method applied to simulations with different uncertainty factors. The simulated radial velocity uncertainties are multiplied with the uncertainty factor before analysing them. The binary fraction $f_{\text {binaries }}=10 \%$ is fixed. 30 simulations per point for statistics. A slightly overestimation of the uncertainties is better for the accuracy of the resulting binary fraction than an underestimation.

But what are the impacts if we make mistakes in our model? What happens if the cluster densities used in simulation and analysis differ from each other? In Fig. A.6 the resulting fraction of analysed and simulated binaries for different unique epochs is shown as a function of the simulated cluster density. As expected, if we underestimate the cluster density the resulting binary fraction is higher, but the resulting fractions agree better to the simulated ones when we do more observations.

On the observational data side, we made a lot of effort to get the radial velocity uncertainties correct. But what happens if we under- or overestimate the uncertainties? In Fig. A.7 the resulting binary fractions for different uncertainty factors are shown, applied to all radial velocity uncertainties. A slight overestimation of the uncertainties is better for the accuracy of the resulting binary fraction than an underestimation.

A big advantage of the model based EM method is that our resulting overall binary fraction is always complete. But for single stars this is another story. A simple assumption to identify a star as a binary star is to evaluate if the radial velocity variability probability for this star is higher than the threshold $P_{\text {binary }}\left(\chi^{2}, \nu\right)>0.5$. In our simulations we know exactly which stars are binaries and which are single stars. In Fig. A.8a and Fig. A.8b the resulting correct and falsepositive binary star detections can be seen as an example for the simulated globular clusters NGC 3201 and NGC 1851. It is noticeable, that for denser clusters (hence shorter periods and larger amplitudes) the number of observations (to get an reasonable sampling) necessary to 


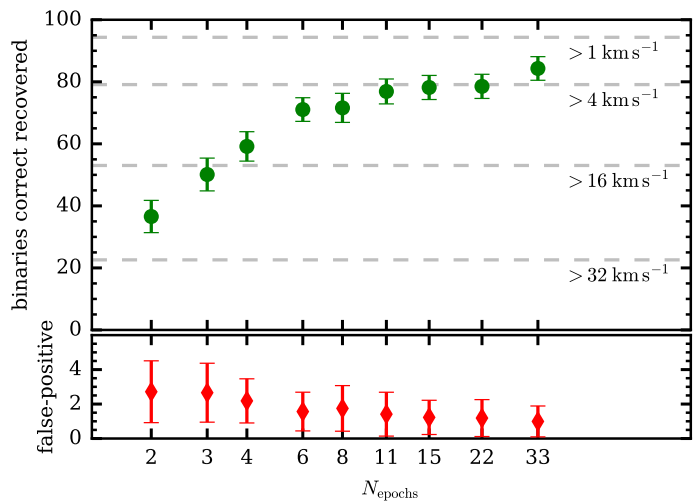

(a) NGC 3201

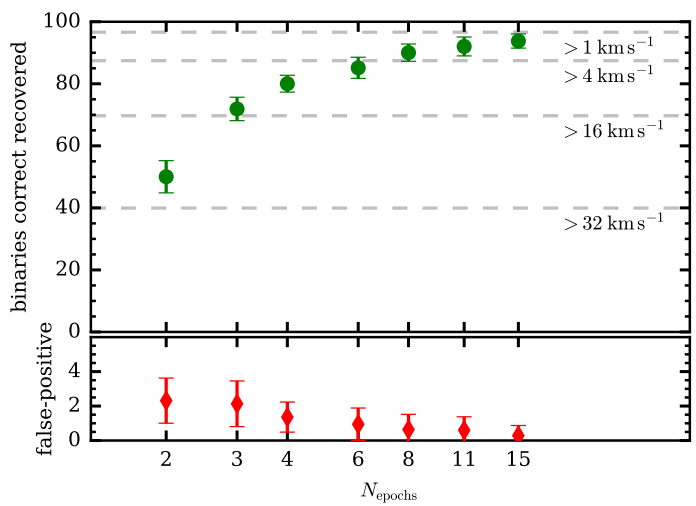

(b) NGC 1851

Figure A.8.: The correct and false-positive binary star detections for different epochs with the expectation maximisation method. Both, the successfully detected binaries in the top panel and the false-positive detections in the bottom panel, are shown in percent as a function of the number of simulated observations $N_{\text {epochs. }}$ The dashed lines indicate sensitivity thresholds to detect radial velocity variations. 100 simulations per point for statistics.

recover the same amount of binaries can be smaller than in less dense clusters.

\section{A.3.3. Results}

A dedicated analysis for all globular clusters like the one in the second paper in Chapter 2 is desirable, but needs a lot of observations and sophisticated models to compare with. The statistical method introduced in the second paper in Chapter 2 was applied to all globular clusters in the MUSE sample. This gives a first impression for individual stars if radial velocity variations are present. However, observational binary fractions are not easy to compare between clusters as shown in the second paper in Chapter 2. Either way, models are necessary to correct for selection effects. Therefore, the combination of the expectation maximisation technique from Sect. A.3.1 and the toy model from Sect. A.2 for globular clusters is used to calculate the binarity in the globular cluster sample.

The membership of the stars is also checked per cluster with the expectation maximisation technique using the metallicity, mean radial velocity, and projected distance to the cluster centre (see Kamann et al., 2016, for more details). A star is considered as a member if it has a higher probability than $50 \%$ to be a cluster member.

To avoid biasing the binary fraction too much by photometric variable stars with radial velocities due to pulsations, we use the differential photometry as described in Sect. A.1 to exclude the obvious cases with a probability higher than $90 \%$ to be photometric variables. Therefore, the majority of stars in our samples showing variations in radial velocity should be binaries. 
A.3. Binary fractions in the MUSE globular cluster sample

Table A.1.: Binary fractions of different stellar types in the MUSE sample with all observed stars $f_{\mathrm{MUSE}}$, stars on the main sequence $f_{\mathrm{MS}}$, and stars on the red giant branch $f_{\mathrm{RGB}}$ in different globular clusters. Additionally, the amount of stars in the blue straggler star region and its binary fraction $f_{\mathrm{BSS}}$ and the binary fraction of the comparison region $f_{\text {Compare }}$ with comparable brightness range are listed.

\begin{tabular}{c|ccc|ccc}
\hline \hline Globular cluster & $f_{\text {MUSE }}[\%]$ & $f_{\text {RGB }}[\%]$ & $f_{\text {MS }}[\%]$ & \#BSS & $f_{\text {BSS }}[\%]$ & $f_{\text {Compare }}[\%]$ \\
\hline NGC 104 & $13.3 \pm 0.3$ & $14.4 \pm 0.8$ & $11.7 \pm 0.3$ & 147 & $30.5 \pm 4.4$ & $16.2 \pm 0.8$ \\
NGC 362 & $10.4 \pm 0.5$ & $11.5 \pm 1.0$ & $7.0 \pm 0.4$ & 37 & $18.9 \pm 6.0$ & $12.0 \pm 1.3$ \\
NGC 1851 & $7.7 \pm 0.4$ & $10.3 \pm 1.0$ & $5.7 \pm 0.4$ & 123 & $13.8 \pm 3.2$ & $8.4 \pm 0.7$ \\
NGC 2808 & $14.2 \pm 0.6$ & $14.7 \pm 0.8$ & - & 68 & $15.2 \pm 3.3$ & $14.5 \pm 0.8$ \\
NGC 3201 & $10.9 \pm 0.5$ & $20.4 \pm 4.2$ & $9.6 \pm 0.5$ & 32 & $68.7 \pm 10.4$ & $18.1 \pm 3.5$ \\
NGC 5139 & $5.1 \pm 0.1$ & $4.4 \pm 0.5$ & $5.6 \pm 0.3$ & 45 & $25.6 \pm 7.5$ & $4.2 \pm 0.6$ \\
NGC 6254 & $5.9 \pm 0.3$ & $6.0 \pm 1.0$ & $5.5 \pm 0.3$ & 35 & $37.2 \pm 9.5$ & $5.4 \pm 1.2$ \\
NGC 6266 & $16.4 \pm 0.8$ & $14.5 \pm 0.8$ & - & - & - & - \\
NGC 6293 & $0.0 \pm 0.0$ & $0.0 \pm 0.0$ & - & - & - & - \\
NGC 6388 & $18.5 \pm 0.5$ & $18.0 \pm 0.7$ & - & 64 & $31.1 \pm 6.2$ & $18.3 \pm 0.8$ \\
NGC 6441 & $20.6 \pm 0.5$ & $18.5 \pm 0.6$ & - & 101 & $24.8 \pm 4.0$ & $17.8 \pm 0.7$ \\
NGC 6522 & $7.4 \pm 0.9$ & $7.1 \pm 1.3$ & - & - & - & - \\
NGC 6681 & $2.2 \pm 0.3$ & $1.1 \pm 0.2$ & $2.3 \pm 0.4$ & - & - & - \\
NGC 6752 & $3.9 \pm 0.2$ & $3.3 \pm 0.8$ & $3.3 \pm 0.2$ & 21 & $6.9 \pm 4.5$ & $4.0 \pm 0.9$ \\
NGC 7078 & $13.5 \pm 0.4$ & $15.3 \pm 1.0$ & $11.8 \pm 0.6$ & 57 & $18.1 \pm 4.2$ & $14.6 \pm 1.1$ \\
NGC 7089 & $13.6 \pm 0.4$ & $13.7 \pm 0.7$ & $11.7 \pm 0.8$ & 122 & $20.8 \pm 3.6$ & $14.8 \pm 0.9$ \\
NGC 7099 & $5.6 \pm 0.3$ & $3.8 \pm 1.0$ & $5.1 \pm 0.3$ & 66 & $19.6 \pm 6.2$ & $4.7 \pm 1.1$ \\
\hline
\end{tabular}

The resulting binary fractions of this analysis for the clusters with more than one epoch in the MUSE sample by May 2017 are listed in Table A.1. In addition to the full star sample of each cluster, the binary fraction is calculated separately for the RGB, MS, BSS, and one to the BSS region comparable star region in the CMD.

A correlation of the observed binary fraction $f_{\text {MUSE }}$ with the absolute visual magnitude $M_{V, t}$ (cluster luminosity) and the core mass of the cluster has been identified. Without $\omega$ Cen the Spearman correlation coefficient is -0.80 for the cluster luminosity and 0.86 for the cluster core mass showing an increase of the binary fraction with increasing cluster luminosity (mass). This correlation is shown in Fig. A.9a, notice that $\omega$ Cen (NGC 5139) is an outlier, which could be due to the special status of this cluster (see Sect. 1.1.1 for more details). This correlation is clearly contradicting literature, that there should be an anti-correlation between binary fraction and cluster luminosity (Milone et al., 2012). Theoretical, luminous clusters with higher core masses normally also have higher core densities which should decrease the binary fractions in that cores over their lifetime. Maybe due to our diverse observational biases and the simple toy model (see the second paper in Chapter 2 as an example for the problems in only one cluster) our result could be opposite. In the end we decided not to publish this result, since too many questions remained unanswered.

The binary fractions of the blue straggler stars, on the other hand, allow a much clearer interpretation. Figure A.9b demonstrates that the binarity of blue stragglers is increased in compar- 


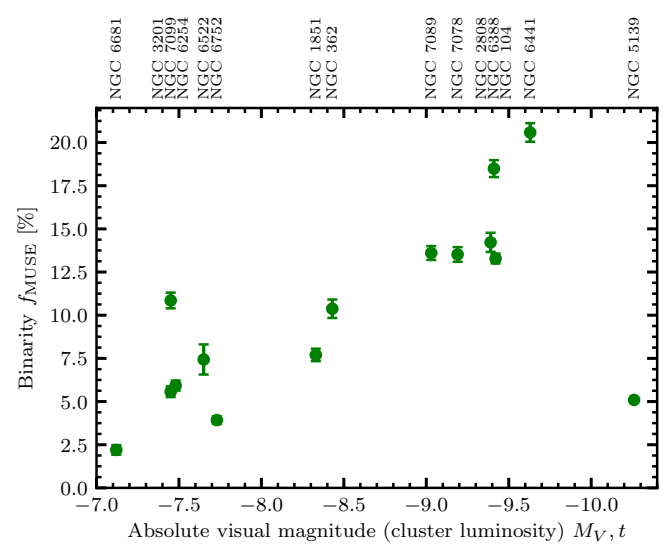

(a) The binary fraction of a globular cluster as a function of its absolute visual magnitude.

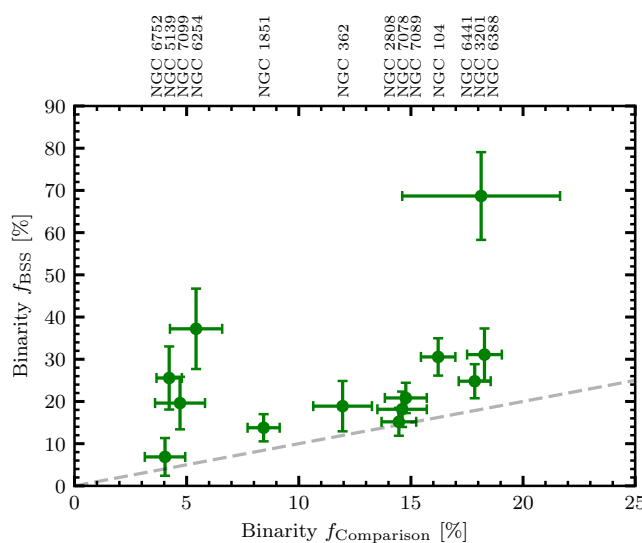

(b) The binary fraction of the blue straggler stars of a globular cluster as a function of a comparison sample with similar brightness. The dotted line represents the identity.

Figure A.9.: Correlations of the binary fractions within the MUSE sample.

ison to a reference sample in all globular clusters. The comparison with the reference sample eliminates most of the systematics. It was already shown in Milone et al. (2012) that the amount of blue stragglers is correlated with the binary fraction of a globular cluster. A further step was taken here, demonstrating that not only the blue straggler binarity is correlated to the cluster binarity, but for most clusters, the blue straggler binarity is significantly increased in contrast to the binarity of the cluster itself. This puts clear constraints on the blue straggler formation scenarios and is discussed in detail in the second paper in Chapter 2 for the globular cluster NGC 3201.

\section{A.4. Matching of literature catalogues to MUSE data}

One of the key ingredients to successfully analyse the binary content in the globular cluster NGC 3201 (see the second paper in Chapter 2 as an example) are the external catalogues we matched with our MUSE observations. Astronomers are used to use archive search engines like the VizieR ${ }^{2}$ or SIMBAD ${ }^{3}$ databases, both maintained by the Centre de Données Astronomiques de Strasbourg (CDS). However, our MUSE survey of globular clusters does contain more than 300000 stars. It would be extremely time consuming to search for individual stars in these databases and double check their position and properties to be sure the correct star is identified. Therefore, it would be easier to match all available information with our sample and hold it in a database. In the following the matching method and literature catalogues we have matched with the MUSE data are presented.

\footnotetext{
${ }^{2}$ VizieR provides the most complete library of published astronomical catalogues.

${ }^{3}$ SIMBAD contains information for stars and nonstellar objects.
} 


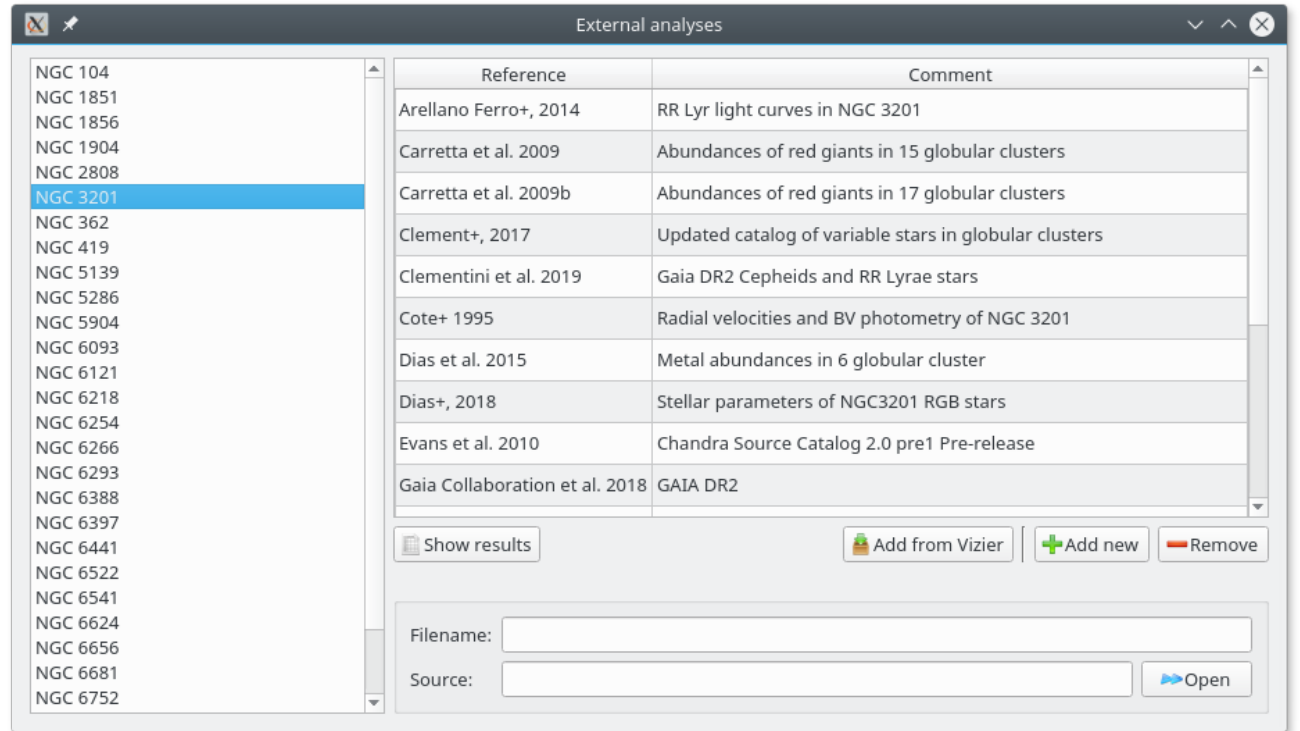

Figure A.10.: User interface to manage literature catalogues for the MUSE globular cluster sample.

\section{A.4.1. Matching method}

The basis for our spectrum extraction from the MUSE observations in most globular clusters is the HST ACS globular cluster survey (Sarajedini et al., 2007, Anderson et al., 2008) catalogue. This is a catalogue consisting of precise HST astrometry and photometry, hereafter called master catalogue. To match external catalogues with our sources, we need at least one visual magnitude and position (right ascension (RA), declination (Dec.)) per star. We designed a tool that gives automatic access to VizieR catalogues. In Fig. A.10 and Fig. A.11 this tool is shown for NGC 3201 as an example. The user can select a VizieR catalogue and it will automatically be downloaded. There is no standard name for the position or magnitude columns in a VizieR table, but the tool tries to identify these columns itself to do a quick check if stars in the catalogue are in common with our master catalogue. Nevertheless, the user can select 'Match with GCDB' to open an interactive matching tool which is shown in Fig. A.12.

This matching tool gives a graphical representation of the matching process which is based on positions and magnitudes of the stars. The coordinate transformation from the selected catalogue to the master catalogue only corrects for offsets. In principle it could also correct for rotation and dilation, but it turned out these effects are less important than a global translation. To find the transformation, the algorithm can use only a fraction of the brightest stars in the sample, which reduces mismatches dramatically. The metric which is minimised during the match is:

$$
\Delta m^{2}+x \times d^{2}
$$




\begin{tabular}{|c|c|c|c|c|c|c|c|}
\hline \multicolumn{3}{|l|}{$\Phi \times$} & \multicolumn{3}{|c|}{ Vizier Catalogues } & \multicolumn{2}{|c|}{$? \vee \wedge \otimes$} \\
\hline$\gg$ Insert cluster name & \multicolumn{5}{|c|}{ NGC 3201} & \multicolumn{2}{|l|}{ 8s Search Vizier } \\
\hline Key & \multicolumn{2}{|c|}{ Reference } & \multicolumn{3}{|c|}{ Description } & Link & $\Delta$ \\
\hline$J / A+A / 445 / 155$ & \multicolumn{2}{|c|}{ Webb+, 2006} & \multicolumn{3}{|c|}{ XMM count-rates in M 55 and NGC 3201} & more info & \\
\hline$J / A+A / 497 / 371$ & \multicolumn{2}{|c|}{ Kravtsov+, 2009} & \multicolumn{3}{|c|}{ UBVI photometry of NGC 3201} & more info & \\
\hline J/AJ/123/279 & \multicolumn{2}{|c|}{ von Braun+, 2002} & \multicolumn{3}{|c|}{ VI Photometry and Variables in NGC 3201} & more info & \\
\hline J/AJ/153/134 & \multicolumn{2}{|c|}{ Sariya+, 2017} & \multicolumn{3}{|c|}{ Proper motions and photometry of stars in NGC 3201} & more info & \\
\hline J/PAZh/22/269 & \multicolumn{2}{|c|}{ Samust, 1996} & \multicolumn{3}{|c|}{ Giant branch and variable stars in NGC 3201} & more info & \\
\hline J/ACA/62/357 & \multicolumn{2}{|c|}{ Zloczewski+, 2012} & \multicolumn{3}{|c|}{ Proper motions in 6 globular clusters } & more info & \\
\hline J/other/RMxAA/50.30 & \multicolumn{2}{|c|}{ Arellano Ferro+, 2014} & \multicolumn{3}{|c|}{ RR Lyr light curves in NGC 3201} & more info & \\
\hline \multicolumn{8}{|l|}{4} \\
\hline \multicolumn{8}{|c|}{ 啇 Get tables for selected catalogue } \\
\hline Name & \multicolumn{2}{|c|}{ RA/Decl? Magnitude? } & \multicolumn{5}{|c|}{ Description } \\
\hline J/AJ/153/134/table3 & False & False & \multicolumn{5}{|c|}{ Membership probability of the known variables in the direction o... } \\
\hline J/AJ/153/134/table 4 & 2881 stars & True & \multicolumn{5}{|c|}{ The membership catalog for NGC 3201} \\
\hline Show table $\mathrm{N}$ & Merge with & & NGC $3201-$ & Match with GCDB & ASave as ex & xternal analysis & \\
\hline
\end{tabular}

Figure A.11.: User interface to manage the VizieR tables of literature catalogues.

with the magnitude difference $\Delta m$ and the transformed distance $d$ (taking the determined offsets into account) of each star in the catalogue to the best fit in the master. A good compromise between magnitude and distance is often found with $x=10$ [mag per arcsec], but depends of course on the quality of the positions and magnitudes given in the selected catalogue. For the final match, the last translation, the specified radius (which is represented by the blue ellipse in the bottom left corner of Fig. A.12) to prevent mismatches, and the metric to determine the best fit are used for each star. The matched catalogue can then be internally merged with other VizieR tables (see 'Merge with' in Fig. A.11) and finally saved to the database with the correct literature reference.

\section{A.4.2. HST photometry}

In addition to the HST ACS globular cluster survey (Sarajedini et al., 2007, Anderson et al., 2008) catalogue (our master catalogue) we matched other available photometries. The preliminary public catalogue release of the HST UV Legacy Survey ('UVLS') of Galactic globular clusters ${ }^{4}$ (Soto et al., 2017, Piotto et al., 2015) has been matched to all our clusters when available. After final publication, the refined catalogue, now named HST UV globular cluster survey ${ }^{5}$ ('HUGS') (Nardiello et al., 2018, Piotto et al., 2015), has been added as well. These additional UV filters are

\footnotetext{
${ }^{4}$ http: //www.astro.uda.cl/public_release/globularclusters41.html

5 https://archive.stsci.edu/prepds/hugs/
} 


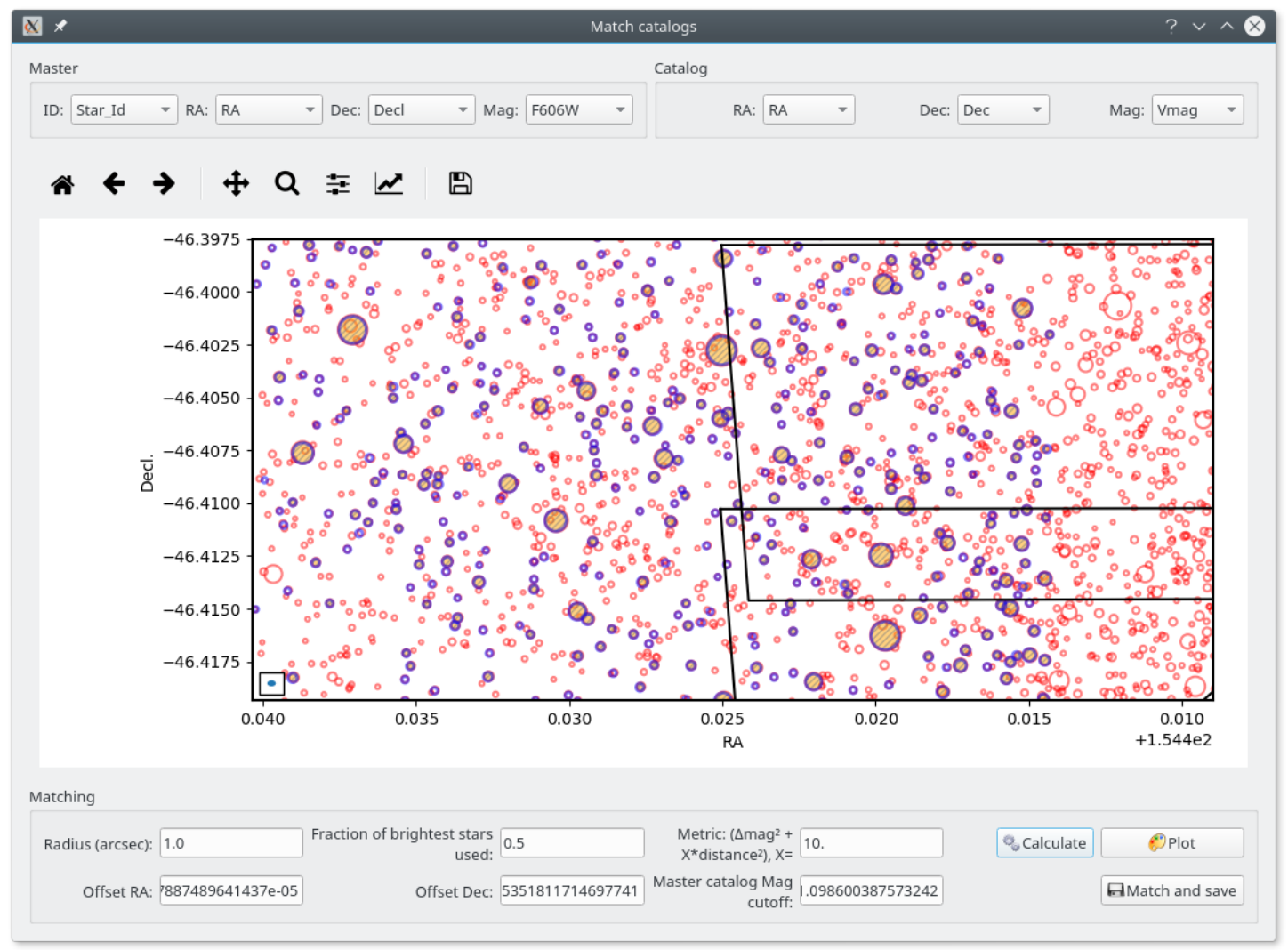

Figure A.12.: User interface to match the stars of a selected VizieR catalogue to the MUSE master catalogue. 
the base for all our multiple populations studies and have for example been used for NGC 3201 in the publications Giesers et al. (2019), Kamann et al. (2020), Göttgens et al. (2019a), Latour et al. (2019), Husser et al. (2020). Since $\omega$ Cen has always been used as a calibration target for all HST instruments and filters, a huge catalogue by Bellini et al. (2017) with 26 filters stretching continuously from $225 \mathrm{~nm}$ in the UV to $1600 \mathrm{~nm}$ in the infrared has been matched to $\omega$ Cen as well.

\section{A.4.3. Proper motions}

Complementary to the MUSE radial velocities (averaged per star) are the proper motions obtainable by high precision astrometry. There is the Hubble Space Telescope Proper Motion (HSTPROMO) program by Bellini et al. (2014) to get astrometry of several globular clusters. Only two catalogues for NGC 362 and NGC 7078 have been made publicly available (Bellini et al., 2014, Libralato et al., 2018). We have matched both with our MUSE data and calculated the three dimensional velocity vector (space velocities) and real stellar velocity from that match. The results of the HSTPROMO program should be treated with caution, because the cluster itself is used as a reference frame, thus neglecting global effects like cluster rotation, which is present in many clusters as shown by, e.g., Kamann et al. (2018b).

\section{A.4.4. Gaia}

The data release 2 (DR2) of Gaia has been matched to the master catalogue of all globular clusters in our sample (Gaia Collaboration et al., 2018). But it was shown in the bachelor thesis by LEON VON HOLT that Gaia is currently too confused in the dense cores of globular clusters and the results are normally not acceptable for our studies. Nevertheless, we identified some runaway stars in some globular clusters which could be interesting to study with improved future Gaia data releases.

\section{A.4.5. List of all matched catalogues}

In Table A.2 all matched catalogues except for the photometries and Gaia DR2, mentioned in the sections before, are listed for the MUSE globular cluster sample.

Table A.2.: Literature catalogues which were matched to the MUSE master catalogue.

\begin{tabular}{ll}
\hline Cluster \& reference & Description \\
\hline \hline NGC 104 & \\
\hline Albrow et al. (2001) & Variables in 47 Tuc \\
Carretta et al. (2009b) & Abundances of red giants in 15 globular clusters \\
Clement (2017) & Updated catalog of variable stars in globular clusters \\
Evans et al. (2010) & Chandra Source Catalog 2.0 pre1 Pre-release \\
Hughes et al. (2007) & CT1T2 Photometry of red giants in 2 globulars
\end{tabular}


Lapenna et al. (2014)

Marino et al. (2016)

Momany et al. (2012)

Wang et al. (2017)

NGC 1851

Carretta et al. (2011)

Clement (2017)

Clementini et al. (2019)

Cummings et al. (2014)

Evans et al. (2010)

Gratton et al. (2012)

Lim et al. (2015)

Marino et al. (2014b)

Scarpa et al. (2011)

Yong et al. (2015)

NGC 1904

Carretta et al. (2009b)

Carretta et al. (2009a)

Clementini et al. (2019)

D'Orazi et al. (2015)

Evans et al. (2010)

Kains et al. (2012)

Scarpa et al. (2011)

NGC 2808

Cacciari et al. (2004)

Clement (2017)

Clementini et al. (2019)

D'Orazi et al. (2015)

Dias et al. (2015)

Evans et al. (2010)

Gratton et al. (2011)

Marino et al. (2014a)

Massari et al. (2016)

Moni Bidin et al. (2011)

Wang et al. (2016b)

\section{NGC 3201}

Arellano Ferro et al. (2014)

Carretta et al. (2009b)

Carretta et al. (2009a)

Clement (2017)

Clementini et al. (2019)

Cote et al. (1995)
AGB and RGB stars in 47 Tuc

47 Tuc sub-giant branch chemical abundances

8.6um imaging of 47 Tuc

NGC 104, 6121 \& 6809 AGB and RGB stars Na abundance

Spectroscopy of 124 RGB stars in NGC 1851

Updated catalog of variable stars in globular clusters

Gaia DR2 Cepheids and RR Lyrae stars

Photometry in globular clusters. I. NGC 1851

Chandra Source Catalog 2.0 pre1 Pre-release

Horizontal branch stars in NGC 1851

Spectroscopic HK' index for 3 globular clusters

Photometry and abundances of NGC1851 stars

Radial velocities in two globular clusters

Line list for the NGC 1851 stars

Abundances of red giants in 15 globular clusters

Abundances of red giants in 17 globular clusters

Gaia DR2 Cepheids and RR Lyrae stars

$\mathrm{Li}$ abundance of giants in 3 globular clusters

Chandra Source Catalog 2.0 pre1 Pre-release

VrI light curves of NGC1904 variables

Radial velocities in two globular clusters

RGB stars in NGC 2808 observed with FLAMES

Updated catalog of variable stars in globular clusters

Gaia DR2 Cepheids and RR Lyrae stars

$\mathrm{Li}$ abundance of giants in 3 globular clusters

Metal abundances in 6 globular cluster

Chandra Source Catalog 2.0 pre1 Pre-release

Horizontal branch stars in NGC 2808

NGC 2808 HB stars abundances

JK photometry of NGC 2808

Radial velocities in the globular NGC 2808

NGC 2808 AGB and RGB stars Na abundance

RR Lyr light curves in NGC 3201

Abundances of red giants in 15 globular clusters

Abundances of red giants in 17 globular clusters

Updated catalog of variable stars in globular clusters

Gaia DR2 Cepheids and RR Lyrae stars

Radial velocities and BV photometry of NGC 3201 
Appendix

Dias et al. (2015)

Dias et al. (2018)

Evans et al. (2010)

Kaluzny et al. (2016)

Kravtsov et al. (2009)

Mucciarelli et al. (2015)

Sariya et al. (2017)

Simunovic \& Puzia (2014)

Zloczewski et al. (2012)

von Braun \& Mateo (2002)
Carretta et al. (2013)

Clement (2017)

Clementini et al. (2019)

D'Orazi et al. (2015)

Evans et al. (2010)

Libralato et al. (2018)

Lim et al. (2016)

Székely et al. (2007)

NGC 5139

Bellini et al. (2009)

Boyer et al. (2008)

Braga et al. (2016)

Clement (2017)

Evans et al. (2010)

Johnson et al. (2008)

Johnson \& Pilachowski (2010)

Kaluzny et al. (1997)

Kaluzny et al. (2004)

Lebzelter \& Wood (2016)

Marino et al. (2011)

Navarrete et al. (2017)

Norris et al. (1996)

Pancino et al. (2007)

Simpson et al. (2012)

Sollima et al. (2004)

Sollima et al. (2005)

Sollima et al. (2006)

Villanova et al. (2014)
Metal abundances in 6 globular cluster

Stellar parameters of NGC3201 RGB stars

Chandra Source Catalog 2.0 pre1 Pre-release

The Clusters AgeS Experiment (CASE). Variable Stars in the Field of the Globular Cluster NGC 3201

UBVI photometry of NGC 3201

Iron EWs for 21 giant star members of NGC3201

Proper motions and photometry of stars in NGC 3201. Cluster distance used: $4.9+-0.1 \mathrm{kpc}$.

Dynamical Properties of Blue Straggler Stars in NGC 3201

Proper motions in 6 globular clusters

VI Photometry and Variables in NGC 3201

Detailed chemical abundances in the GlC NGC 362

Updated catalog of variable stars in globular clusters

Gaia DR2 Cepheids and RR Lyrae stars

$\mathrm{Li}$ abundance of giants in 3 globular clusters

Chandra Source Catalog 2.0 pre1 Pre-release

Proper Motion (HSTPROMO) catalog of NGC 362

$\mathrm{HK}^{\prime}, \mathrm{CN} \& \mathrm{CH}$ chemical indexes in NGC 362 \& NGC 6723

Variables stars in NGC 362

UBV(RI)cHalpha photometry in omega Cen

omega Cen Spitzer photometry

RRLs in globulars. IV. UBVRI photometry in Omega Cen

Updated catalog of variable stars in globular clusters

Chandra Source Catalog 2.0 pre1 Pre-release

Abundances of red giants in Omega Cen

Omega Centauri giants abundances

RR Lyr stars in omega Cen

Variable Stars in the field of omega Cen

Long period variables in omega Cen

Spectroscopy of 300 RGBs in omega Cen

omega Cen RR Lyrae and SX Phoenicis stars

Ca abundance for Omega Cen red giants

Radial velocities RGB stars in omega Cen

Stellar parameters of giants in omega Cen

JK photometry of Omega Cen red giants

BVI photometry of omega Cen red giants

Metallicities of RR Lyrae stars in Omega Cen

Parameters of NGC 5139 SGBs stars 
van Loon et al. (2007) Spectral atlas of omega Cen post-MS stars

\begin{tabular}{|c|c|}
\hline \multicolumn{2}{|l|}{ NGC 5286} \\
\hline Alvarado et al. (1995) & UBVRI sequences in globulars \\
\hline Clement (2017) & Updated catalog of variable stars in globular clusters \\
\hline Clementini et al. (2019) & Gaia DR2 Cepheids and RR Lyrae stars \\
\hline Evans et al. (2010) & Chandra Source Catalog 2.0 pre1 Pre-release \\
\hline Marino et al. (2015) & Red giants in NGC 5286 \\
\hline Zorotovic et al. (2010) & Variable stars in NGC 5286 \\
\hline \multicolumn{2}{|l|}{ NGC 5904} \\
\hline Carretta et al. (2009b) & Abundances of red giants in 15 globular clusters \\
\hline Clement (2017) & Updated catalog of variable stars in globular clusters \\
\hline Clementini et al. (2019) & Gaia DR2 Cepheids and RR Lyrae stars \\
\hline Evans et al. (2010) & Chandra Source Catalog 2.0 pre1 Pre-release \\
\hline Lai et al. (2011) & M5 chemical abundances for evolved stars \\
\hline Lanzoni et al. (2007) & Blue straggler population of globular cluster M5 \\
\hline Rees (1993) & Proper motions in M5 \\
\hline Sandquist \& Bolte (2004) & BVI photometry of M5 giant branch stars (AGB) \\
\hline Sandquist \& Bolte (2004) & BVI photometry of M5 giant branch stars (RGB) \\
\hline Sandquist \& Bolte (2004) & BVI photometry of M5 giant branch stars (RR Lyrae) \\
\hline Sandquist \& Bolte (2004) & BVI photometry of M5 giant branch stars (HB) \\
\hline Vivas et al. (2017) & CTIO/DECam photometry of RR Lyrae stars in M5 \\
\hline Warren et al. (2006) & HST photometry of M5 blue stragglers \\
\hline \multicolumn{2}{|l|}{ NGC 6093} \\
\hline Carretta et al. (2015) & Abundances of red giant stars in NGC6093 (M80) \\
\hline Clement (2017) & Updated catalog of variable stars in globular clusters \\
\hline Clementini et al. (2019) & Gaia DR2 Cepheids and RR Lyrae stars \\
\hline Dieball et al. (2010) & $\begin{array}{l}\text { A far-ultraviolet survey of M80: X-ray source counterparts, strange } \\
\text { blue stragglers, and ... }\end{array}$ \\
\hline Evans et al. (2010) & Chandra Source Catalog 2.0 pre1 Pre-release \\
\hline Kopacki (2013) & V-band light curves of variables in M80 \\
\hline \multicolumn{2}{|l|}{ NGC 6121} \\
\hline Carretta et al. (2009b) & Abundances of red giants in 15 globular clusters \\
\hline Carretta et al. (2009a) & Abundances of red giants in 17 globular clusters \\
\hline Clement (2017) & Updated catalog of variable stars in globular clusters \\
\hline Clementini et al. (2019) & Gaia DR2 Cepheids and RR Lyrae stars \\
\hline Dias et al. (2015) & Metal abundances in 6 globular cluster \\
\hline Evans et al. (2010) & Chandra Source Catalog 2.0 pre1 Pre-release \\
\hline Malavolta et al. (2015) & $\begin{array}{l}\text { Internal kinematics from accurate radial velocities of } 2771 \text { cluster } \\
\text { members in M4 (NGC 6121). }\end{array}$ \\
\hline Malavolta et al. (2014) & Atmospheric parameters of M4 stars \\
\hline Malavolta et al. (2015) & M4 Core Project with HST. Radial velocities \\
\hline Marino et al. (2008) & Abundances of NGC 6121 red giants \\
\hline
\end{tabular}


Appendix

Mochejska et al. (2002)

Nascimbeni et al. (2014)

Neeley et al. (2015)

Peterson et al. (1995)

Stetson et al. (2014)

Stetson et al. (2014)

Zloczewski et al. (2012)

NGC 6218

Carretta et al. (2007a)

Evans et al. (2010)

Zloczewski et al. (2012)

NGC 6254

Carretta et al. (2009b)

Carretta et al. (2009a)

Clement (2017)

Dias et al. (2015)

Pollard et al. (2005)

Pollard et al. (2005)

NGC 6266

Clementini et al. (2019)

Contreras et al. (2010)

Evans et al. (2010)

Lapenna et al. (2015a)

NGC 6293

Clement (2017)

Clementini et al. (2019)

Evans et al. (2010)

\section{NGC 6388}

Clement (2017)

Clementini et al. (2019)

Dalessandro et al. (2008)

Evans et al. (2010)

Hughes et al. (2007)

Lanzoni et al. (2013)

Lapenna et al. (2015b)

Lützgendorf et al. (2011b)

NGC 6397

Clement (2017)

Evans et al. (2010)

NGC 6441

Clement (2017)

Clementini et al. (2019)
M4 UBV color-magnitude diagrams

Variable stars in M4 primary field

RR Lyrae stars in M4. II. Spitzer/IRAC phot.

Radial Velocities of Stars for M4 members

UBVRIJHK photometry of RR Lyrae in M4

UBVRIJHK photometry of other variables in M4

Proper motions in 6 globular clusters

Abundances of red giants in NGC 6218

Chandra Source Catalog 2.0 pre1 Pre-release

Proper motions in 6 globular clusters

Abundances of red giants in 15 globular clusters

Abundances of red giants in 17 globular clusters

Updated catalog of variable stars in globular clusters

Metal abundances in 6 globular cluster

CCD BVIc photometry of M10 stars: BVI

CCD BVIc photometry of M10 stars: BI

\section{Gaia DR2 Cepheids and RR Lyrae stars}

RR Lyrae in M62 (NGC6266)

Chandra Source Catalog 2.0 pre1 Pre-release

M62 (NGC 6266) giant branch stars abundances

Updated catalog of variable stars in globular clusters

Gaia DR2 Cepheids and RR Lyrae stars

Chandra Source Catalog 2.0 pre1 Pre-release

Updated catalog of variable stars in globular clusters

Gaia DR2 Cepheids and RR Lyrae stars

Blue stragglers in NGC 6388

Chandra Source Catalog 2.0 pre1 Pre-release

CT1T2 Photometry of red giants in 2 globulars

$\mathrm{RVs}$ and $[\mathrm{Fe} / \mathrm{H}]$ of star members of NGC 6388

Radial velocities of giant stars in NGC 6388

VI photometry and velocity map of NGC 6388

Updated catalog of variable stars in globular clusters

Chandra Source Catalog 2.0 pre1 Pre-release

Updated catalog of variable stars in globular clusters

Gaia DR2 Cepheids and RR Lyrae stars 
A.4. Matching of literature catalogues to MUSE data

Dias et al. (2015) Metal abundances in 6 globular cluster

Evans et al. (2010) Chandra Source Catalog 2.0 pre1 Pre-release

NGC 6522

Clement (2017)

Clementini et al. (2019)

Updated catalog of variable stars in globular clusters

Evans et al. (2010)

Gaia DR2 Cepheids and RR Lyrae stars

NGC 6541

Clement (2017) Updated catalog of variable stars in globular clusters

Evans et al. (2010) Chandra Source Catalog 2.0 pre1 Pre-release

NGC 6656

Arp \& Melbourne (1959) Photometry in the globular cluster M22

Clement (2017)

Clementini et al. (2019)

Updated catalog of variable stars in globular clusters

Dias et al. (2015)

Evans et al. (2010)

Gaia DR2 Cepheids and RR Lyrae stars

Gratton et al. (2014)

Metal abundances in 6 globular cluster

Chandra Source Catalog 2.0 pre1 Pre-release

Kaluzny \& Thompson (2001)

Horizontal branch stars in M22

Kaluzny \& Thompson (2001)

SX Phe stars in M22 globular cluster

Lee (2015)

New variables in M22 globular cluster

Marino et al. (2012)

Ca,by photometry in globular clusters. I. M22

Roederer et al. (2011)

Abundances of M22 subgiants

Salgado et al. (2013)

Abundances of six RGB stars in M22

Zloczewski et al. (2012)

NGC6656 spectroscopy of 71 blue HB stars

NGC 6681

Clement (2017)

Proper motions in 6 globular clusters

Evans et al. (2010)

Updated catalog of variable stars in globular clusters

NGC 6752

Carretta et al. (2009a)

Chandra Source Catalog 2.0 pre1 Pre-release

Carretta et al. (2007b)

Abundances of red giants in 17 globular clusters

Clement (2017)

Abundances of red giants in NGC 6752

Evans et al. (2010)

Kravtsov et al. (2014)

Updated catalog of variable stars in globular clusters

Thomson et al. (2012)

Chandra Source Catalog 2.0 pre1 Pre-release

UBVI photometry in NGC 6752

NGC 6752 multiwavelength survey

Zloczewski et al. (2012)

Proper motions in 6 globular clusters

\section{NGC 7078}

Bellini et al. (2014)

Carretta et al. (2009b)

Clement (2017)

Clementini et al. (2019)

Dias et al. (2015)

Díaz-Sánchez et al. (2012)

Proper motion observations of NGC 7078 (HSTPROMO I). Cluster distance used: 10.4+-0.1 kpc.

Abundances of red giants in 15 globular clusters

Updated catalog of variable stars in globular clusters

Gaia DR2 Cepheids and RR Lyrae stars

Metal abundances in 6 globular cluster

VI photometry of M15 core 
Appendix

Evans et al. (2010)

Chandra Source Catalog 2.0 pre1 Pre-release

Gebhardt et al. (2000)

CFHT adaptive optics observations of M15

NGC 7089

Clement (2017)

Updated catalog of variable stars in globular clusters

Clementini et al. (2019)

Gaia DR2 Cepheids and RR Lyrae stars

Dalessandro et al. (2009)

Blue stragglers in M2

Evans et al. (2010)

Chandra Source Catalog 2.0 pre1 Pre-release

Lardo et al. (2012)

U,V photometry in M2 (NGC 7089)

NGC 7099

Carretta et al. (2009b)

Abundances of red giants in 15 globular clusters

Carretta et al. (2009a)

Abundances of red giants in 17 globular clusters

Clement (2017)

Clementini et al. (2019)

Updated catalog of variable stars in globular clusters

Evans et al. (2010)

Gaia DR2 Cepheids and RR Lyrae stars

Gruyters et al. (2016)

Chandra Source Catalog 2.0 pre1 Pre-release

Kains et al. (2013)

Stellar parameters and abundances for M30

Lovisi et al. (2013b)

VrI light curves of NGC7099 variables

Blue straggler and red giant stars in M30

Scarpa et al. (2007)

Radial velocities in NGC 7099 


\section{A.5. Stellar rotation of blue straggler stars}

Simplified, light coming from half of the resolved disk of a rotating star with its rotation axis perpendicular to the line of sight is blueshifted and from the other half of the disk redshifted. In a spectrum this results in a broadening of all spectral absorption lines. Of course, the inclination of the rotation axis, limb darkening, and differential rotation of the star play a role. But in first approximation, the stellar rotation velocity $V \sin i$ (not inclination corrected) could be recovered from a spectrum if the main parameters effecting the line shapes - temperature, metallicity, surface gravity, and line spread function of the spectrograph - are known. A full spectrum fit with SPEXXY can also fit the additional broadening $\sigma$ of the lines in parallel to temperature, surface gravity, (total integrated) radial velocity, and metallicity. Usually we fix the surface gravity to the value found by an isochrone comparison, since the spectral resolution of MUSE is not high enough to fit surface gravity for the typical globular cluster stars. For stellar rotation, a higher spectral resolution also helps a lot, but we found that a MUSE spectrum with a $S / N>20$ and a stellar rotation velocity of $V \sin i>30 \mathrm{~km} \mathrm{~s}^{-1}$ could be fitted reliably. Most stars in old globular clusters should have stellar rotation velocities below our sensitivity, mainly because of their age and stellar type (Skumanich, 1972). But as explained in Sect. 1.1.4, blue straggler stars could have been formed by stellar collisions less than $200 \mathrm{Myr}$ ago. In most cases these blue straggler stars could have a strong rotation velocity up to $V \sin i>50 \mathrm{~km} \mathrm{~s}^{-1}$.

\section{A.6. A systematic way to normalise the continuum in a spectrum}

A stellar spectrum is usually a combination of the stellar black body radiation, atomic emission lines, atomic absorption lines, molecular absorption bands in the stellar and Earth atmosphere, and noise. Sometimes it is helpful to normalise the spectrum, i.e. remove the continuum from the spectrum, for example to calculate line ratios. In the following, a general systematic way is shown to normalise a spectrum.

In a spectrum, that has already been normalised, all data points in the spectrum near unity (not equal due to noise) are points basically unaffected by absorption or emission features, thus continuum points. Here, cohesive continuum points, uninterrupted by spectral features, are called continuum bunches. These continuum bunches could be identified in non-normalised spectra using three conditions:

1. a minimum number of cohesive continuum points in a continuum bunch (e.g. 3),

2. a maximum min to max (peak to peak) variation of continuum point fluxes within a continuum bunch (e.g. 1\%), and

3. a maximum change of flux between two continuum bunches (e.g. $5 \%$ ). 


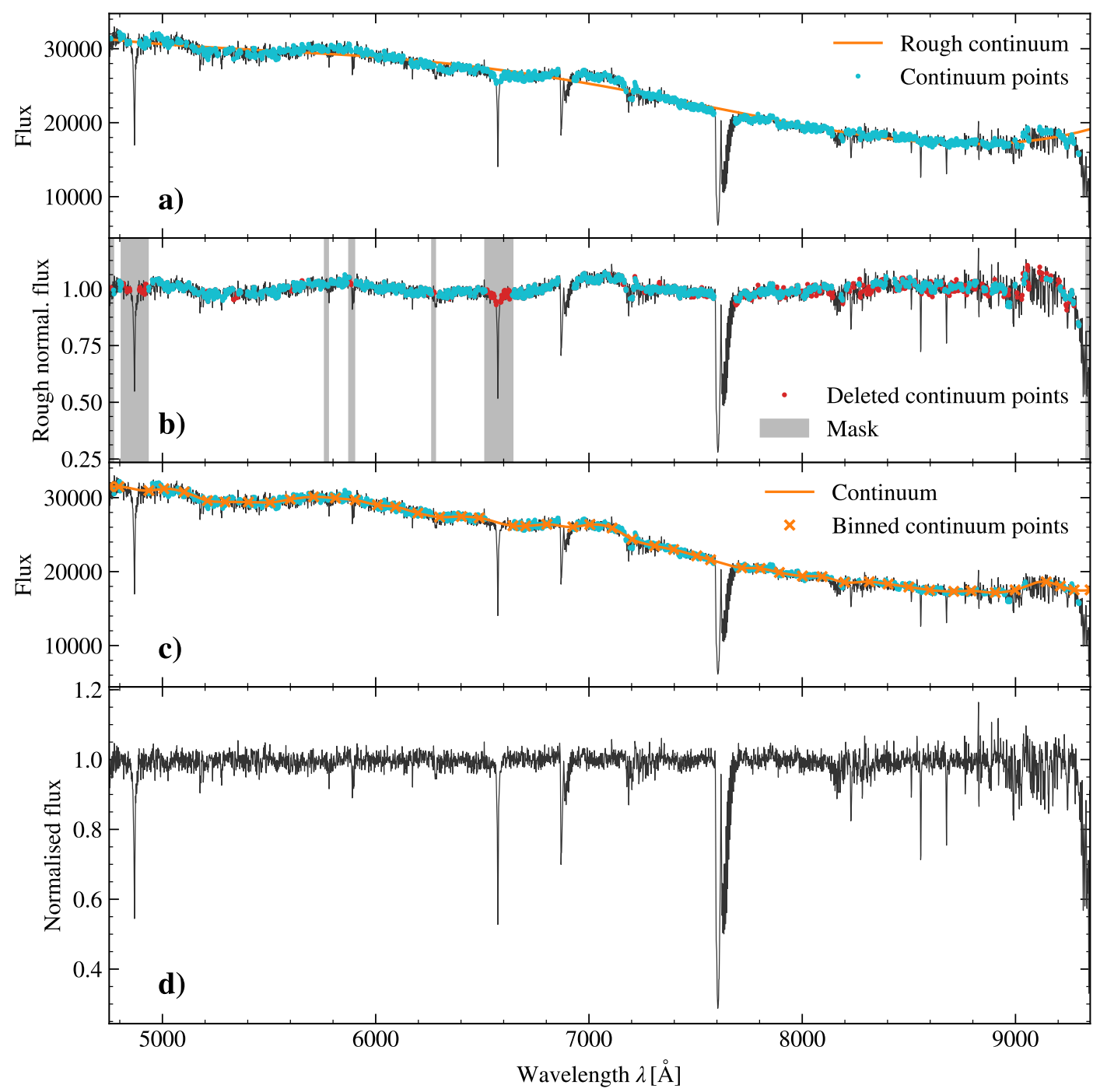

Figure A.13.: Normalisation of a spectrum, more details in text. Panel a) shows the continuum bunches that fulfil conditions (1) to (2). In panel b) a rough normalisation has been applied to check for condition (3) iteratively. In panel c) the continuum points in the original spectrum are binned to suppress noise and fitted with a spline interpolator. Finally, in panel d) the normalised spectrum is shown.

To find continuum bunches in a spectrum, the algorithm scans the flux along the wavelength axis and tries to find cohesive flux values which fulfil the conditions (1) and (2). After the bunches have been identified, the median of every bunch can be compared with the median of their neighbouring bunches and tested with condition (3). The last step has to be done iteratively, until it converges. 
After the bunches are identified, a binning of continuum points along the wavelength axis (e.g. 100 continuum points per bin, with the mean in wavelength and median in flux dimension) is helpful to suppress noise. Finally, the continuum could be interpolated from these bins by using for example a spline interpolator (e.g. the UnivariateSpline interpolator from the scipy package by Jones et al., 2001-2019).

For the cases, when there are steep continuum variations in the spectrum, it is helpful to use this algorithm in two stages. The top panel of Fig. A.13 shows the first stage, in which a rough continuum with less restrictive parameters for the conditions (1) to (3) has been identified. Continued with a rough normalisation with for example a polynom of fourth degree (orange curve in the top panel). The panel b) of Fig. A.13 shows the second stage, in which a finer continuum on this pre-normalised spectrum with refined parameters for the conditions (1) to (3) is determined. Note the final binning and interpolation is done on the original spectrum (see panel c) in Fig. A.13), the rough normalisation is only an auxiliary step to identify the wavelength points of the continuum. It turned out, that this two stages approach can be used reliably for most cases ${ }^{6}$. The bottom panel of Fig. A.13 shows a normalised example spectrum. Of course, the boundary conditions could be improved, but all in all this normalisation method works well. It was used to normalise the spectra in the first and second publication in Chapter 2 (Giesers et al., 2018, 2019) and Göttgens et al. (2019a).

${ }^{6} \mathrm{An}$ exception are spectra of M-stars with steep changes in molecular bands, which are difficult to normalise. 



\section{Bibliography}

Aвbott, B. P., Aвbott, R., Abbott, T. D., Abernathy, M. R., Acernese, F., Ackley, K., ADAMS, C., ADAMS, T. ET AL. (2016) Observation of Gravitational Waves from a Binary Black Hole Merger. Physical Review Letters, 116(6), 061102.

Albrow, M. D., Gilliland, R. L., Brown, T. M., Edmonds, P. D., GuhathaKurTa, P. \& SARAJEDINI, A. (2001) The Frequency of Binary Stars in the Core of 47 Tucanae. The Astrophysical Journal, 559(2), 1060-1081.

Alessandrini, E., Lanzoni, B., Ferraro, F. R., Miocchi, P. \& Vesperini, E. (2016) Investigating the Mass Segregation Process in Globular Clusters with Blue Straggler Stars: The Impact of Dark Remnants. ApJ, 833(2), 252.

Alvarado, F., Wenderoth, E., Alcaino, G. \& Liller, W. (1995) Photoelectric UBVRI Sequences in the Galactic Globular Clusters NGC 5286, NGC 5927, and NGC 6541. The Astronomical Journal, 109, 1169.

Anderson, J., Sarajedini, A., Bedin, L. R., King, I. R., Piotto, G., Reid, I. N., Siegel, M., MAJEWSKI, S. R. ET AL. (2008) The Acs Survey of Globular Clusters. V. Generating a Comprehensive Star Catalog for each Cluster. AJ, 135, 2055-2073.

Arca Sedda, M., Askar, A. \& GIERSZ, M. (2018) MOCCA-Survey Database - I. Unravelling black hole subsystems in globular clusters. MNRAS, 479, 4652-4664.

Arellano Ferro, A., Ahumada, J. A., CAlderón, J. H. \& Kains, N. (2014) Fourier Decomposition of RR Lyrae light curves and the SX Phe population in the central region of NGC 3201. Revista Mexicana de Astronomia y Astrofisica, 50, 307-322.

ARP, H. C., BAUM, W. A. \& SANDAGE, A. R. (1952) The HR diagrams for the globular clusters M 92 and M 3. AJ, 57, 4-5.

ARP, H. C. \& Melbourne, W. G. (1959) Color-magnitude diagram for the globular cluster M 22. The Astronomical Journal, 64, 28-32.

AsHMAN, K. M. \& ZEPF, S. E. (1992) The formation of globular clusters in merging and interacting galaxies. ApJ, 384, 50-61.

AsKar, A., Arca SEDdA, M. \& Giersz, M. (2018) MOCCA-SURVEY Database I: Galactic globular clusters harbouring a black hole subsystem. MNRAS, 478, 1844-1854. 
Askar, A., Askar, A., Pasquato, M. \& Giersz, M. (2019) Finding black holes with black boxes - using machine learning to identify globular clusters with black hole subsystems. MNRAS, 485, 5345-5362.

Askar, A., Szkudlarek, M., Gondek-Rosińska, D., Giersz, M. \& BuliK, T. (2017) MOCCA-SURVEY Database - I. Coalescing binary black holes originating from globular clusters. MNRAS, 464, L36-L40.

Bacon, R., Accardo, M., Adjali, L., Anwand, H., Bauer, S., Biswas, I., Blaizot, J., BOUdON, D. ET AL. (2010) The MUSE second-generation VLT instrument. In Ground-based and Airborne Instrumentation for Astronomy III, vol. 7735 of Proc. SPIE.

BAILYN, C. D. (1992) Are There Two Kinds of Blue Stragglers in Globular Clusters? ApJ, 392, 519.

BAILYN, C. D. (1995) Blue Stragglers and Other Stellar Anomalies:Implications for the Dynamics of Globular Clusters. ARAEA, 33, 133-162.

BANERJEE, S. (2018) Stellar-mass black holes in young massive and open stellar clusters and their role in gravitational-wave generation III: dissecting black hole dynamics. MNRAS, 481, 5123-5145.

BAstian, N. \& LARDO, C. (2018) Multiple Stellar Populations in Globular Clusters. ARA\&A, 56, 83-136.

Bastian, N., Saglia, R. P., Goudfrooij, P., Kissler-Patig, M., Maraston, C., SchWEIZER, F. \& ZOCCALI, M. (2006) Dynamical mass estimates for two luminous star clusters in galactic merger remnants. $A \& A, 448(3), 881-891$.

BAUMGARDT, H. (2017) N -body modelling of globular clusters: masses, mass-to-light ratios and intermediate-mass black holes. MNRAS, 464, 2174-2202.

BAUmgardT, H. \& MAKINO, J. (2003) Dynamical evolution of star clusters in tidal fields. MNRAS, 340(1), 227-246.

BAumgardT, H. \& Sollima, S. (2017) The global mass functions of 35 Galactic globular clusters - II. Clues on the initial mass function and black hole retention fraction. MNRAS, $472,744-750$.

Beccari, G., Dalessandro, E., Lanzoni, B., Ferraro, F. R., Sollima, A., Bellazzini, M. \& Miocchi, P. (2013) Deep Multi-telescope Photometry of NGC 5466. I. Blue Stragglers and Binary Systems. ApJ, 776(1), 60.

Beccari, G., Ferraro, F. R., Dalessandro, E., Lanzoni, B., Raso, S., Origlia, L., VesPERINI, E., HONG, J. ET AL. (2019) Discovery of a Double Blue Straggler Sequence in M15: New Insight into the Core-collapse Process. ApJ, 876(1), 87. 
Bedin, L. R., Piotto, G., Anderson, J., CAssisi, S., King, I. R., Momany, Y. \& CARraro, G. (2004) $\omega$ Centauri: The Population Puzzle Goes Deeper. ApJ, 605(2), L125-L128.

Belczynski, K., SAdOWsKi, A., RAsio, F. A. \& Bulik, T. (2006) Initial Populations of Black Holes in Star Clusters. ApJ, 650, 303-325.

Bellini, A., Anderson, J., Bedin, L. R., King, I. R., VAn Der Marel, R. P., Piotto, G. \& CoOL, A. (2017) The State-of-the-art HST Astro-photometric Analysis of the Core of $\omega$ Centauri. I. The Catalog. ApJ, 842, 6.

Bellini, A., Anderson, J., VAn der Marel, R. P., Watkins, L. L., King, I. R., BianchINI, P., Chanamé, J., Chandar, R. ET AL. (2014) Hubble Space Telescope Proper Motion (HSTPROMO) Catalogs of Galactic Globular Clusters. I. Sample Selection, Data Reduction, and NGC 7078 Results. ApJ, 797, 115.

Bellini, A., Piotto, G., Bedin, L. R., Anderson, J., Platais, I., Momany, Y., Moretti, A., Milone, A. P. ET AL. (2009) Ground-based CCD astrometry with wide field imagers. III. WFI@2.2m proper-motion catalog of the globular cluster $\omega$ Centauri. Astronomy and Astrophysics, 493(3), 959-978.

Benson, J. A., Hutter, D. J., Elias, N. M., I., Bowers, P. F., Johnston, K. J., Hajian, A. R., Armstrong, J. T., Mozurkewich, D. ET AL. (1997) Multichannel optical aperture synthesis imaging of zeta1 URSAE majoris with the Navy prototype optical interferometer. AJ, 114, 1221-1226.

Binney, J. \& Tremaine, S. (2008) Galactic Dynamics: Second Edition. Princeton University Press.

BOMBACI, I. (1996) The maximum mass of a neutron star. $A \mathcal{E} A, \mathbf{3 0 5}, 871$.

Boyer, M. L., McDonald, I., van Loon, J. T., Woodward, C. E., Gehrz, R. D., Evans, A. \& Dupree, A. K. (2008) A Spitzer Space Telescope Atlas of $\omega$ Centauri: The Stellar Population, Mass Loss, and the Intracluster Medium. The Astronomical Journal, 135(4), 1395-1411.

Braga, V. F., Stetson, P. B., Bono, G., Dall'Ora, M., Ferraro, I., Fiorentino, G., FREYHAMMER, L. M., IANNICOLA, G. ET AL. (2016) On the RR Lyrae Stars in Globulars. IV. $\omega$ Centauri Optical UBVRI Photometry. The Astronomical Journal, 152(6), 170.

Cacciari, C., Bragaglia, A., Rossetti, E., Fusi Pecci, F., Mulas, G., Carretta, E., Gratton, R. G., MOMAnY, Y. ET AL. (2004) Mass motions and chromospheres of RGB stars in the globular cluster NGC 2808. Astronomy and Astrophysics, 413, 343-362.

CARR, B. J. (1975) The primordial black hole mass spectrum. ApJ, 201, 1-19.

Carretta, E., Bragaglia, A., Gratton, R. \& Lucatello, S. (2009a) Na-O anticorrelation and HB. VIII. Proton-capture elements and metallicities in 17 globular clusters from UVES spectra. Astronomy and Astrophysics, 505(1), 139-155. 
Carretta, e., Bragaglia, A., Gratton, R. G., Catanzaro, G., Leone, F., Sabbi, E., CASSISI, S., ClAUdi, R. ET AL. (2007a) Na-O anticorrelation and horizontal branches. IV. Detection of He-rich and He-poor stellar populations in the globular cluster NGC 6218. A\&A, 464(3), 939-951.

Carretta, E., Bragaglia, A., Gratton, R. G., D'Orazi, V., Lucatello, S., Sollima, A., Momany, Y., Catanzaro, G. ET AL. (2015) The normal chemistry of multiple stellar populations in the dense globular cluster NGC 6093 (M 80). Astronomy and Astrophysics, 578, A116.

Carretta, E., Bragaglia, A., Gratton, R. G., Lucatello, S., Catanzaro, G., Leone, F., Bellazzini, M., ClAUdi, R. ET AL. (2009b) Na-O anticorrelation and HB. VII. The chemical composition of first and second-generation stars in 15 globular clusters from GIRAFFE spectra. $A \mathcal{E} A, \mathbf{5 0 5}(1), 117-138$.

Carretta, E., Bragaglia, A., Gratton, R. G., Lucatello, S., D'Orazi, V., Bellazzini, M., CATANZARO, G., LEONE, F. ET AL. (2013) NGC 362: another globular cluster with a split red giant branch. Astronomy and Astrophysics, 557, A138.

Carretta, E., Bragaglia, A., Gratton, R. G., Lucatello, S. \& Momany, Y. (2007b) Na$\mathrm{O}$ anticorrelation and horizontal branches. II. The $\mathrm{Na}-\mathrm{O}$ anticorrelation in the globular cluster NGC 6752. Astronomy and Astrophysics, 464(3), 927-937.

Carretta, E., Gratton, R. G., Lucatello, S., Bragaglia, A., Catanzaro, G., LeOne, F., Momany, Y., D'OrAZI, V. ET AL. (2010) Abundances for a Large Sample of Red Giants in NGC 1851: Hints for a Merger of Two Clusters? ApJ, 722, L1-L6.

Carretta, E., Lucatello, S., Gratton, R. G., Bragaglia, A. \& D'Orazi, V. (2011) Multiple stellar populations in the globular cluster NGC 1851. Astronomy and Astrophysics, 533, A69.

Carroll, B. W. \& Ostlie, D. A. (2017) An Introduction to Modern Astrophysics. 2nd edn., Cambridge University Press.

Catalán, S., Isern, J., García-Berro, E. \& Ribas, I. (2008) The initial-final mass relationship of white dwarfs revisited: effect on the luminosity function and mass distribution. MNRAS, 387, 1693-1706.

Chomiuk, L., Strader, J., Maccarone, T. J., Miller-Jones, J. C. A., Heinke, C., NoyOLA, E., SeTH, A. C. \& RANSOM, S. (2013) A Radio-selected Black Hole X-Ray Binary Candidate in the Milky Way Globular Cluster M62. ApJ, 777, 69.

Clement, C. M. (2017) VizieR Online Data Catalog: Updated catalog of variable stars in globular clusters (Clement+ 2017). VizieR Online Data Catalog, 5150. 
Clement, C. M., Muzzin, A., Dufton, Q., Ponnampalam, T., Wang, J., Burford, J., Richardson, A., Rosebery, T. ET AL. (2001) Variable Stars in Galactic Globular Clusters. AJ, 122, 2587-2599.

Clementini, G., Ripepi, V., Molinaro, R., Garofalo, A., Muraveva, T., Rimoldini, L., GuY, L. P., Jevardat De Fombelle, G. et Al. (2019) Gaia Data Release 2. Specific characterisation and validation of all-sky Cepheids and RR Lyrae stars. Astronomy and Astrophysics, 622, A60.

Contreras, R., Catelan, M., Smith, H. A., Pritzl, B. J., Borissova, J. \& Kuehn, C. A. (2010) Time-series Photometry of Globular Clusters: M62 (NGC 6266), the Most RR Lyrae-rich Globular Cluster in the Galaxy? The Astronomical Journal, 140(6), 1766-1786.

Cote, P., Welch, D. L., Fischer, P. \& Gebhardt, K. (1995) Dynamics of the Galactic Globular Cluster NGC 3201. The Astrophysical Journal, 454, 788.

Cummings, J. D., Geisler, D., Villanova, S. \& Carraro, G. (2014) Uncovering Multiple Populations with Washington Photometry. I. The Globular Cluster NGC 1851. The Astronomical Journal, 148(2), 27.

Dalessandro, E., Beccari, G., LAnzoni, B., Ferraro, F. R., Schiavon, R. \& RoOd, R. T. (2009) Multiwavelength Photometry in the Globular Cluster M2. The Astrophysical Journal Supplement Series, 182(2), 509-518.

Dalessandro, E., Lanzoni, B., Ferraro, F. R., Rood, R. T., Milone, A., Piotto, G. \& VAlENTI, E. (2008) Blue Straggler Stars in the Unusual Globular Cluster NGC 6388. The Astrophysical Journal, 677(2), 1069-1079.

Dalessandro, E., Mucciarelli, A., Bellazzini, M., Sollima, A., Vesperini, E., Hong, J., Hénault-Brunet, V., Ferraro, F. R. et al. (2018) The Unexpected Kinematics of Multiple Populations in NGC 6362: Do Binaries Play a Role? ApJ, 864(1), 33.

Davies, M. B., Piotto, G. \& De Angeli, F. (2004) Blue straggler production in globular clusters. MNRAS, 349(1), 129-134.

DE GRIJS, R. (2010) A revolution in star cluster research: setting the scene. Philosophical Transactions of the Royal Society of London Series A, 368(1913), 693-711.

DE GRIJS, R. \& LI, C. (2018) Not-so-simple stellar populations in nearby, resolved massive star clusters. Phys. Scr, 93(2), 024001.

Dias, B., Araya, I., Nogueira-Cavalcante, J. P., Saker, L. \& Shokry, A. (2018) Galactic or extragalactic chemical tagging for NGC 3201?. Discovery of an anomalous CN-CH relation. Astronomy and Astrophysics, 614, A146. 
Dias, B., Barbuy, B., Saviane, I., Held, E. V., Da Costa, G. S., Ortolani, S., Vasquez, S., GUllieusZIK, M. ET AL. (2015) FORS2/VLT survey of Milky Way globular clusters. I. Description of the method for derivation of metal abundances in the optical and application to NGC 6528, NGC 6553, M 71, NGC 6558, NGC 6426, and Terzan 8. Astronomy and Astrophysics, 573, A13.

díaz-Sánchez, A., Pérez-Garrido, A., Villó, I., Rebolo, R., Pérez-Prieto, J. A., Oscoz, A., Hildebrand T, S. R., López, R. eT Al. (2012) High-resolution optical imaging of the core of the globular cluster M15 with FastCam. Monthly Notices of the Royal Astronomical Society, 423(3), 2260-2269.

Dieball, A., Long, K. S., Knigge, C., Thomson, G. S. \& Zurek, D. R. (2010) A Farultraviolet Survey of M80: X-Ray Source Counterparts, Strange Blue Stragglers, and the Recovery of Nova T Sco. The Astrophysical Journal, 710(1), 332-345.

D’Orazi, V., Gratton, R., Lucatello, S., Carretta, E., Bragaglia, A. \& Marino, A. F. (2010) Ba Stars and Other Binaries in First and Second Generation Stars in Globular Clusters. ApJ, 719, L213-L217.

D’Orazi, V., Gratton, R. G., Angelou, G. C., Bragaglia, A., Carretta, E., lattanzio, J. C., Lucatello, S., Momany, Y. ET AL. (2015) Lithium abundances in globular cluster giants: NGC 1904, NGC 2808, and NGC 362. Monthly Notices of the Royal Astronomical Society, 449(4), 4038-4047.

Dotter, A., Sarajedini, A., Anderson, J., Aparicio, A., Bedin, L. R., Chaboyer, B., MAJEWSKI, S., MARÍN-FrAnCH, A. ET AL. (2010) The ACS Survey of Galactic Globular Clusters. IX. Horizontal Branch Morphology and the Second Parameter Phenomenon. ApJ, 708, 698-716.

DuQuenNoy, A. \& MAYOR, M. (1991) Multiplicity among solar-type stars in the solar neighbourhood. II - Distribution of the orbital elements in an unbiased sample. A\&A, 248, 485-524.

Eisenhauer, F., Abuter, R., Bickert, K., Biancat-Marchet, F., Bonnet, H., Brynnel, J., CONZelmanN, R. D., Delabre, B. ET AL. (2003) SINFONI - Integral field spectroscopy at 50 milli-arcsecond resolution with the ESO VLT. In Instrument Design and Performance for Optical/Infrared Ground-based Telescopes, edited by M. Iye \& A. F. M. Moorwood, vol. 4841 of Proc. SPIE.

Evans, I. N., Primini, F. A., Glotfelty, K. J., Anderson, C. S., Bonaventura, N. R., Chen, J. C., Davis, J. E., Doe, S. M. ET AL. (2010) The Chandra Source Catalog. ApJS, 189, $37-82$.

Event Horizon Telescope Collaboration, Akiyama, K., Alberdi, A., Alef, W., AsADA, K., Azulay, R., BACZKO, A.-K., BAll, D. ET Al. (2019) First M87 Event Horizon Telescope Results. I. The Shadow of the Supermassive Black Hole. ApJ, 875, L1. 
Fabian, A. C., Pringle, J. E. \& Rees, M. J. (1975) Tidal capture formation of binary systems and X-ray sources in globular clusters. MNRAS, 172,15p-18p.

Ferraro, F. R., Beccari, G., Dalessandro, E., Lanzoni, B., Sills, A., Rood, R. T., PECCI, F. F., KARAKAS, A. I. ET AL. (2009) Two distinct sequences of blue straggler stars in the globular cluster M 30. Nature, 462(7276), 1028-1031.

Ferraro, F. R. \& LAnzoni, B. (2009) Probing the link between dynamics and stellar evolution: Blue Straggler Stars in Globular clusters. In Revista Mexicana de Astronomia y Astrofisica Conference Series, vol. 37.

Ferraro, F. R., Lanzoni, B., Dalessandro, E., Beccari, G., Pasquato, M., Miocchi, P., RoOD, R. T., SigurdSSON, S. ET AL. (2012) Dynamical age differences among coeval star clusters as revealed by blue stragglers. Nature, 492, 393-395.

Ferraro, F. R., Sabbi, E., Gratton, R., Piotto, G., Lanzoni, B., Carretta, E., Rood, R. T., SILlS, A. ET AL. (2006) Discovery of Carbon/Oxygen-depleted Blue Straggler Stars in 47 Tucanae: The Chemical Signature of a Mass Transfer Formation Process. ApJ, 647, L53-L56.

Freeman, K. \& Bland-HAWthorn, J. (2002) The New Galaxy: Signatures of Its Formation. ARAEA, 40, 487-537.

Fregeau, J. M., Cheung, P., Portegies Zwart, S. F. \& Rasio, F. A. (2004) Stellar collisions during binary-binary and binary-single star interactions. MNRAS, 352, 1-19.

Fregeau, J. M., IVANova, N. \& RAsio, F. A. (2009) Evolution of the Binary Fraction in Dense Stellar Systems. ApJ, 707, 1533-1540.

FROST, E. B. (1908) On certain spectroscopic binaries. Astronomische Nachrichten, 177(11), 171.

Gaia Collaboration, Brown, A. G. A., Vallenari, A., Prusti, T., De Bruijne, J. H. J., Babusiaux, C., BAiler-Jones, C. A. L., Biermann, M. et Al. (2018) Gaia Data Release 2. Summary of the contents and survey properties. $A \mathcal{E} A, \mathbf{6 1 6}$, A1.

Gaia Collaboration, Prusti, T., de Bruijne, J. H. J., Brown, A. G. A., Vallenari, A., Babusiaux, C., Bailer-Jones, C. A. L., Bastian, U. et Al. (2016) The Gaia mission. AEA, 595, A1.

GaO, B., Goodman, J., COHN, H. \& Murphy, B. (1991) Fokker-Planck calculations of star clusters with primordial binaries. ApJ, 370, 567-582.

Gebhardt, K., Pryor, C., O’Connell, R. D., Williams, T. B. \& Hesser, J. E. (2000) Canada-France-Hawaii Telescope Adaptive Optics Observations of the Central Kinematics in M15. The Astronomical Journal, 119(3), 1268-1281. 
Geller, A. M., Leiner, E. M., Bellini, A., Gleisinger, R., Haggard, D., Kamann, S., Leigh, N. W. C., Mathieu, R. D. ET AL. (2017a) On the Origin of Sub-subgiant Stars. I. Demographics. ApJ, 840, 66.

Geller, A. M., Leiner, E. M., Chatterjee, S., Leigh, N. W. C., Mathieu, R. D. \& Sills, A. (2017b) On the Origin of Sub-subgiant Stars. III. Formation Frequencies. ApJ, 842, 1.

Gieles, M., Charbonnel, C., Krause, M. G. H., Hénault-Brunet, V., Agertz, O., LAmers, H. J. G. L. M., BAstian, N., GUAlandris, A. ET AL. (2018) Concurrent formation of supermassive stars and globular clusters: implications for early self-enrichment. MNRAS, $478,2461-2479$.

Giersz, M. (1998) Monte Carlo simulations of star clusters - I. First Results. MNRAS, 298(4), 1239-1248.

GIERsZ, M. (2006) Monte Carlo simulations of star clusters - III. A million-body star cluster. MNRAS, 371(1), 484-494.

Giersz, M., Heggie, D. C., Hurley, J. R. \& HyPKI, A. (2013) MOCCA code for star cluster simulations - II. Comparison with N-body simulations. MNRAS, 431(3), 2184-2199.

Giesers, B., Dreizler, S., Husser, T.-O., Kamann, S., Anglada Escudé, G., BrinchMANN, J., CAROLLO, C. M., Roth, M. M. ET AL. (2018) A detached stellar-mass black hole candidate in the globular cluster NGC 3201. MNRAS, 475, L15-L19.

Giesers, B., Kamann, S., Dreizler, S., Husser, T.-O., AsKar, A., Göttgens, F., BrinchMANN, J., LATOUR, M. ET AL. (2019) A stellar census in globular clusters with MUSE: Binaries in NGC 3201. A\&A, 632, A3.

Giesler, M., CLAusen, D. \& OTT, C. (2017) The population of low-mass X-ray binaries ejected from black-hole retaining globular clusters. In APS April Meeting Abstracts.

Goodman, J. \& Hut, P. (1989) Primordial binaries and globular cluster evolution. Nature, 339, $40-42$.

Göttgens, F., Husser, T.-O., Kamann, S., Dreizler, S., Giesers, B., Kollatschny, W., Weilbacher, P. M., Roth, M. M. ET AL. (2019a) A stellar census in globular clusters with MUSE: A spectral catalogue of emission-line sources. $A \& A, 631$, A118.

Göttgens, F., Weilbacher, P. M., Roth, M. M., Dreizler, S., Giesers, B., Husser, T.O., KAMANN, S., BRINCHMANN, J. ET AL. (2019b) Discovery of an old nova remnant in the Galactic globular cluster M 22. A\&A, 626, A69.

Gratton, R., Sneden, C. \& Carretta, E. (2004) Abundance Variations Within Globular Clusters. ARAEA, 42(1), 385-440. 
Gratton, R. G., Bonifacio, P., Bragaglia, A., Carretta, E., Castellani, V., CenturION, M., ChIEFFI, A., ClAudi, R. ET AL. (2001) The O-Na and Mg-Al anticorrelations in turn-off and early subgiants in globular clusters. $A \mathcal{E} A, 369,87-98$.

Gratton, R. G., Lucatello, S., Carretta, E., Bragaglia, A., D'Orazi, V., Al Momany, Y., SOllima, A., SAlaris, M. ET AL. (2012) The Na-O anticorrelation in horizontal branch stars. II. NGC 1851. Astronomy and Astrophysics, 539, A19.

Gratton, R. G., Lucatello, S., Carretta, E., Bragaglia, A., D'Orazi, V. \& Momany, Y. A. (2011) The Na-O anticorrelation in horizontal branch stars. I. NGC 2808. Astronomy and Astrophysics, 534, A123.

Gratton, R. G., Lucatello, S., Sollima, A., Carretta, E., Bragaglia, A., Momany, Y., D'ORAZI, V., CASSISI, S. ET AL. (2014) The Na-O anticorrelation in horizontal branch stars. IV. M 22. Astronomy and Astrophysics, 563, A13.

Gravity Collaboration, Abuter, R., Amorim, A., Anugu, N., Bauböck, M., Benisty, M., BERGER, J. P., BLIND, N. ET AL. (2018) Detection of the gravitational redshift in the orbit of the star S2 near the Galactic centre massive black hole. A\&A, 615, L15.

Greenstein, J. L. (1939) Magnitudes and Colors in the Globular Cluster Messier 4. ApJ, 90, 387.

Gruyters, P., Lind, K., Richard, O., Grundahl, F., Asplund, M., Casagrande, L., Charbonnel, C., Milone, A. ET AL. (2016) Atomic diffusion and mixing in old stars. VI. The lithium content of M30. Astronomy and Astrophysics, 589, A61.

GU, W.-M., MU, H.-J., FU, J.-B., ZHENG, L.-L., YI, T., BAI, Z.-R., WANG, S., ZHANG, H.T. ET AL. (2019) A Method to Search for Black Hole Candidates with Giant Companions by LAMOST. ApJ, 872, L20.

GUNN, J. E. \& GRIFFIN, R. F. (1979) Dynamical studies of globular clusters based on photoelectric radial velocities of individual stars. I - M3. AJ, 84, 752-773.

HANSEN, B. M. S. \& PHINNEY, E. S. (1997) The pulsar kick velocity distribution. MNRAS, 291, 569.

HARRIS, W. E. (1996) A Catalog of Parameters for Globular Clusters in the Milky Way. AJ, 112, 1487.

Hearnshaw, J. B. (2014) The Analysis of Starlight: Two Centuries of Astronomical Spectroscopy. 2nd edn., Cambridge University Press.

HeGgIE, D. C. (1975) Binary evolution in stellar dynamics. MNRAS, 173, 729-787. 
Hénon, M. H. (1971) The Monte Carlo Method (Papers appear in the Proceedings of IAU Colloquium No. 10 Gravitational N-Body Problem (ed. by Myron Lecar), R. Reidel Publ. Co. , Dordrecht-Holland.). ApESS, 14(1), 151-167.

Hong, J., Vesperini, E., Sollima, A., McMillan, S. L. W., D'Antona, F. \& D’Ercole, A. (2015) Evolution of binary stars in multiple-population globular clusters. MNRAS, 449, $629-638$.

Hong, J., Vesperini, E., Sollima, A., McMillan, S. L. W., D'Antona, F. \& D’Ercole, A. (2016) Evolution of binary stars in multiple-population globular clusters - II. Compact binaries. MNRAS, 457, 4507-4514.

Hughes, J., Wallerstein, G., Covarrubias, R. \& Hays, N. (2007) Washington Photometry of the Red Giant Branch in NGC 6388. The Astronomical Journal, 134(1), 229-240.

Husser, T.-O., Kamann, S., Dreizler, S., Wendt, M., Wulff, N., Bacon, R., WisotzKi, L., BRINCHMANN, J. ET AL. (2016) MUSE crowded field 3D spectroscopy of over 12000 stars in the globular cluster NGC 6397. I. The first comprehensive HRD of a globular cluster. $A \mathcal{E} A$, 588, A148.

Husser, T.-O., Latour, M., Brinchmann, J., Dreizler, S., Giesers, B., Göttgens, F., KAMANN, S., ROTH, M. M. ET AL. (2020) A stellar census in globular clusters with MUSE. Extending the CaT-metallicity relation below the horizontal branch and applying it to multiple populations. $A \mathcal{E} A, 635$, A114.

Husser, T.-O., Wende-von Berg, S., Dreizler, S., Homeier, D., Reiners, A., Barman, T. \& HAuschildt, P. H. (2013) A new extensive library of PHOENIX stellar atmospheres and synthetic spectra. $A \mathcal{E} A, 553, \mathrm{~A} 6$.

Hut, P., McMillan, S., Goodman, J., Mateo, M., Phinney, E. S., Pryor, C., Richer, H. B., VERBUnt, F. ET AL. (1992) Binaries in globular clusters. PASP, 104, 981-1034.

HYPKI, A. \& GIERSZ, M. (2013) MOCCA code for star cluster simulations - I. Blue stragglers, first results. MNRAS, 429(2), 1221-1243.

HYPKI, A. \& GIERSZ, M. (2017a) MOCCA code for star cluster simulations - V. Initial globular cluster conditions influence on blue stragglers. MNRAS, 466(1), 320-339.

HYPKI, A. \& GIERSZ, M. (2017b) mocca code for star cluster simulations - VI. Bimodal spatial distribution of blue stragglers. MNRAS, 471(3), 2537-2552.

ibata, R. A., Bellazzini, M., Malhan, K., Martin, N. \& Bianchini, P. (2019) Identification of the long stellar stream of the prototypical massive globular cluster $\omega$ Centauri. Nature Astronomy, 3, 667-672. 
IVANOVA, N., Belczynski, K., FregeAu, J. M. \& RAsio, F. A. (2005) The evolution of binary fractions in globular clusters. MNRAS, 358, 572-584.

Ivanova, N., Heinke, C. O., Rasio, F. A., Belczynski, K. \& Fregeau, J. M. (2008) Formation and evolution of compact binaries in globular clusters - II. Binaries with neutron stars. MNRAS, 386, 553-576.

Johnson, C. I. \& Pilachowski, C. A. (2010) Chemical Abundances for 855 Giants in the Globular Cluster Omega Centauri (NGC 5139). The Astrophysical Journal, 722(2), 1373-1410.

Johnson, C. I., Pilachowski, C. A., Simmerer, J. \& Schwenk, D. (2008) Fe and Al Abundances for 180 Red Giants in the Globular Cluster Omega Centauri (NGC 5139). The Astrophysical Journal, 681(2), 1505-1523.

Jones, E., Oliphant, T., Peterson, P. et Al. (2001-2019) SciPy: Open source scientific tools for Python. URL http: / / www. scipy • org/, [Online; accessed <today >].

Kains, N., Bramich, D. M., Arellano Ferro, A., Figuera Jaimes, R., Jørgensen, U. G., Giridhar, S., Penny, M. T., Alsubai, K. A. Et Al. (2013) Estimating the parameters of globular cluster M 30 (NGC 7099) from time-series photometry. Astronomy and Astrophysics, 555, A36.

Kains, N., Bramich, D. M., Figuera Jaimes, R., Arellano Ferro, A., Giridhar, S. \& KUPPUSWAMY, K. (2012) Constraining the parameters of globular cluster NGC 1904 from its variable star population. Astronomy and Astrophysics, 548, A92.

Kaluzny, J., Kubiak, M., Szymanski, M., UdAlski, A., KrZeminski, W. \& MateO, M. (1997) The optical gravitational lensing experiment. Variable stars in globular clusters. III. RR Lyrae stars and Pop. II Cepheids in omega Centauri. Astronomy and Astrophysics Supplement Series, 125, 343-353.

Kaluzny, J., Olech, A., Thompson, I. B., Pych, W., KRZEMińsKi, W. \& SchWARZenberGCZERNY, A. (2004) Cluster AgeS ExperimentCatalog of variable stars in the globular cluster $\omega$ Centauri. Astronomy and Astrophysics, 424, 1101-1110.

Kaluzny, J., RozyczKa, M., Thompson, I. B., Narloch, W., Mazur, B., Pych, W. \& SChWARZEnberG-CZERnY, A. (2016) The Clusters AgeS Experiment (CASE). Variable Stars in the Field of the Globular Cluster NGC 3201. Acta Astron., 66, 31-53.

Kaluzny, J. \& Thompson, I. B. (2001) Clusters AgeS Experiment. New variable stars in the globular cluster M 22. Astronomy and Astrophysics, 373, 899-904.

Kamann, S., Bastian, N., Husser, T.-O., Martocchia, S., Usher, C., Den Brok, M., Dreizler, S., Kelz, A. eT AL. (2018a) Cluster kinematics and stellar rotation in NGC 419 with MUSE and adaptive optics. MNRAS, 480, 1689-1695. 
Kamann, S., Giesers, B., Bastian, N., Brinchmann, J., Dreizler, S., Göttgens, F., Husser, T. O., LATOUR, M. ET AL. (2020) The binary content of multiple populations in NGC 3201. A\&A, 635, A65.

Kamann, S., Husser, T.-O., Brinchmann, J., Emsellem, E., Weilbacher, P. M., WisOtZKI, L., WendT, M., KRAJNOvić, D. ET AL. (2016) MUSE crowded field 3D spectroscopy of over 12000 stars in the globular cluster NGC 6397. II. Probing the internal dynamics and the presence of a central black hole. $A \mathcal{E} A, 588, \mathrm{~A} 149$.

Kamann, S., Husser, T.-O., Dreizler, S., Emsellem, E., Weilbacher, P. M., Martens, S., BACON, R., DEN BROK, M. ET AL. (2018b) A stellar census in globular clusters with MUSE: The contribution of rotation to cluster dynamics studied with 200000 stars. MNRAS, 473, $5591-5616$.

KAMANN, S., WiSOTZKI, L. \& ROTH, M. M. (2013) Resolving stellar populations with crowded field 3D spectroscopy. A\&A, 549, A71.

KERN, J. R. \& BOOKMYER, B. B. (1986) Differential photometry of HDE 310376, a rapid variable star. PASP, 98, 1336-1341.

KING, I. R. (1966) The structure of star clusters. III. Some simple dynamical models. AJ, 71, 64.

KING, I. R., Sosin, C. \& CoOL, A. M. (1995) Mass Segregation in the Globular Cluster NGC 6397. ApJ, 452, L33.

KIRCH, G. (1682) Gottfridi Kirchii Annus II. Ephemeridum Motuum Coelestium Ad Annum Aerae Christianae M. DC. LXXXII.: Cum ortu \& Occasu diurno Planetarum, Occultationibusq[ue] tam Planetarum, quam illustriorum Stellarum Fixarum \&c. Ex Tabulis Rudolphinis, Ad Meridianum Uranoburgicum, In Freto Cimbrico Supputatus, Cum Appendice Observationum Nonnullarum Astronomicarum. Kirch, Gottfried; Friedrich Lanckischens Erben; Johann Bauers Witwe, Leipzig.

Knigge, C., Leigh, N. \& Sills, A. (2009) A binary origin for 'blue stragglers' in globular clusters. Nature, 457(7227), 288-290.

KOPACKI, G. (2013) Variable Stars in the Globular Cluster M 80. Acta Astronomica, 63(1), 91-107.

Kormendy, J. \& Ho, L. C. (2013) Coevolution (Or Not) of Supermassive Black Holes and Host Galaxies. ARAEA, 51(1), 511-653.

KRAfT, R. P. (1994) Abundance Differences among Globular Cluster Giants: Primordial vs. Evolutionary Scenarios. PASP, 106, 553.

Kravtsov, V., Alcaíno, G., Marconi, G. \& Alvarado, F. (2009) Multi-color photometry in wide field of the Galactic globular cluster NGC 3201. Astronomy and Astrophysics, 497(2), 371-378. 
Kravtsov, V., Alcaíno, G., Marconi, G. \& Alvarado, F. (2014) Galactic Globular Cluster NGC 6752 and Its Stellar Population as Inferred from Multicolor Photometry. The Astrophysical Journal, 783(1), 56.

Kremer, K., Chatterjee, S., Breivik, K., Rodriguez, C. L., Larson, S. L. \& Rasio, F. A. (2018a) LISA Sources in Milky Way Globular Clusters. Physical Review Letters, 120(19), 191103.

Kremer, K., Chatterjee, S., Ye, C. S., Rodriguez, C. L. \& Rasio, F. A. (2019) How Initial Size Governs Core Collapse in Globular Clusters. ApJ, 871, 38.

Kremer, K., Ye, C. S., Chatterjee, S., Rodriguez, C. L. \& Rasio, F. A. (2018b) How Black Holes Shape Globular Clusters: Modeling NGC 3201. ApJ, 855, L15.

Kremer, K., Ye, C. S., Chatterjee, S., Rodriguez, C. L. \& Rasio, F. A. (2020) The Role of "black hole burning" in the evolution of dense star clusters. In IAU Symposium, edited by A. Bragaglia, M. Davies, A. Sills \& E. Vesperini, vol. 351 of IAU Symposium.

KROUPA, P. (2001) On the variation of the initial mass function. MNRAS, 322(2), 231-246.

KRUIJSSEN, J. M. D. (2012) On the fraction of star formation occurring in bound stellar clusters. MNRAS, 426(4), 3008-3040.

Kruijssen, J. M. D., Pfeffer, J. L., Reina-Campos, M., Crain, R. A. \& Bastian, N. (2019) The formation and assembly history of the Milky Way revealed by its globular cluster population. MNRAS, 486(3), 3180-3202.

KulKarni, S. R., Hut, P. \& MCMillan, S. (1993) Stellar black holes in globular clusters. Nature, 364(6436), 421-423.

KuČinsKas, A., Dobrovolskas, V. \& Bonifacio, P. (2014) Galactic globular cluster 47 Tucanae: new ties between the chemical and dynamical evolution of globular clusters? $A \mathcal{E} A$, $568, \mathrm{~L} 4$.

Lai, D. K., Smith, G. H., Bolte, M., Johnson, J. A., Lucatello, S., Kraft, R. P. \& SNEDEN, C. (2011) Chemical Abundances for Evolved Stars in M5: Lithium through Thorium. The Astronomical Journal, 141(2), 62.

Lanzoni, B., Dalessandro, E., Ferraro, F. R., Mancini, C., Beccari, G., Rood, R. T., MAPELli, M. \& SigurdSSON, S. (2007) The Blue Straggler Population of the Globular Cluster M5. The Astrophysical Journal, 663(1), 267-276.

Lanzoni, B., Ferraro, F. R., Alessandrini, E., Dalessand ro, E., Vesperini, E. \& Raso, S. (2016) Refining the Dynamical Clock for Star Clusters. ApJ, 833(2), L29. 
Lanzoni, B., Mucciarelli, A., Origlia, L., Bellazzini, M., Ferraro, F. R., Valenti, E., Miocchi, P., Dalessandro, E. ET AL. (2013) The Velocity Dispersion Profile of NGC 6388 from Resolved-star Spectroscopy: No Evidence of a Central Cusp and New Constraints on the Black Hole Mass. ApJ, 769, 107.

Lapenna, E., Mucciarelli, A., Ferraro, F. R., Origlia, L., Lanzoni, B., Massari, D. \& Dalessandro, E. (2015a) Chemical Analysis of Asymptotic Giant Branch Stars in M62. The Astrophysical Journal, 813(2), 97.

Lapenna, E., Mucciarelli, A., Lanzoni, B., Ferraro, F. R., Dalessandro, E., Origlia, L. \& MASSARI, D. (2014) Non-local Thermodynamical Equilibrium Effects on the Iron Abundance of Asymptotic Giant Branch Stars in 47 Tucanae. The Astrophysical Journal, 797(2), 124.

Lapenna, E., Origlia, L., Mucciarelli, A., Lanzoni, B., Ferraro, F. R., Dalessandro, E., VAlenti, E. \& CirAsuolo, M. (2015b) Radial Velocities from VLT-KMOS Spectra of Giant Stars in the Globular Cluster NGC 6388. The Astrophysical Journal, 798(1), 23.

Lardo, C., Bellazzini, M., Pancino, E., Carretta, E., Bragaglia, A. \& Dalessandro, E. (2011) Mining SDSS in search of multiple populations in globular clusters. $A \mathcal{E} A$, 525, A114.

Lardo, C., Pancino, E., Mucciarelli, A. \& Milone, A. P. (2012) Carbon and nitrogen abundances of stellar populations in the globular cluster M 2. Astronomy and Astrophysics, 548, A107.

Larsen, S. S., Baumgardt, H., Bastian, N., Brodie, J. P., Grundahl, F. \& Strader, J. (2015) Radial Distributions of Sub-Populations in the Globular Cluster M15: A More Centrally Concentrated Primordial Population. ApJ, 804, 71.

Latour, M., Husser, T. O., Giesers, B., Kamann, S., Göttgens, F., Dreizler, S., BRINCHMANN, J., BASTIAN, N. ET AL. (2019) A stellar census in globular clusters with MUSE: multiple populations chemistry in NGC 2808. A\&A, 631, A14.

Lebzelter, T. \& Wood, P. R. (2016) The long-period variables in $\omega$ Centauri. Astronomy and Astrophysics, 585, A111.

LEE, J.-W. (2015) Multiple Stellar Populations of Globular Clusters from Homogeneous Ca by Photometry. I. M22 (NGC 6656). The Astrophysical Journal Supplement Series, 219(1), 7.

Lee, Y. W., JoO, J. M., Sohn, Y. J., ReY, S. C., Lee, H. C. \& WAlKer, A. R. (1999) Multiple stellar populations in the globular cluster $\omega$ Centauri as tracers of a merger event. Nature, 402(6757), 55-57.

Leibundgut, B., Bacon, R., Jaffé, Y. L., Johnston, E., Kuntschner, H., Selman, F., VAlenti, E., Vernet, J. ET AL. (2017) MUSE WFM AO Science Verification. The Messenger, 170, 20-25. 
Leigh, N., Giersz, M., Webb, J. J., Hypki, A., De Marchi, G., Kroupa, P. \& Sills, A. (2013) The state of globular clusters at birth: emergence from the gas-embedded phase. MNRAS, 436, 3399-3412.

Leigh, N. W. C., Giersz, M., Marks, M., Webb, J. J., Hypki, A., Heinke, C. O., Kroupa, P. \& SILLS, A. (2015) The state of globular clusters at birth - II. Primordial binaries. MNRAS, 446, 226-239.

Leigh, N. W. C., Panurach, T., Simunovic, M., Geller, A. M., Zurek, D., Shara, M. M., Sills, A., KNIGGE, C. ET AL. (2019) Constraints on blue straggler formation mechanisms in galactic globular clusters from proper motion velocity distributions. MNRAS, 482, 231-239.

Leiner, E., Mathieu, R. D., Gosnell, N. M. \& Sills, A. (2018) Observations of Spin-down in Post-mass-transfer Stars and the Possibility for Blue Straggler Gyrochronology. ApJ, 869(2), L29.

LeVERINGTON, D. (1995) A History of Astronomy: from 1890 to the Present. Springer, London, London.

LI, C., DE GRIJS, R. \& DenG, L. (2014) Not-so-simple Stellar Populations in the Intermediateage Large Magellanic Cloud Star Clusters NGC 1831 and NGC 1868. ApJ, 784(2), 157.

Libralato, M., Bellini, A., van der Marel, R. P., Anderson, J., Watkins, L. L., Piotto, G., Ferraro, F. R., Nardiello, D. et Al. (2018) Hubble Space Telescope Proper Motion (HSTPROMO) Catalogs of Galactic Globular Cluster. VI. Improved Data Reduction and Internal-kinematic Analysis of NGC 362. ApJ, 861, 99.

Lim, D., Han, S.-I., LeE, Y.-W., RoH, D.-G., Sohn, Y.-J., Chun, S.-H., LeE, J.-W. \& JOHNSON, C. I. (2015) Low-resolution Spectroscopy for the Globular Clusters with Signs of Supernova Enrichment: M22, NGC 1851, and NGC 288. The Astrophysical Journal Supplement Series, 216(1), 19.

Lim, D., Lee, Y.-W., Pasquato, M., HaN, S.-I. \& RoH, D.-G. (2016) Reversed Trend of Radial Distribution of Subpopulations in the Globular Clusters NGC 362 and NGC 6723. The Astrophysical Journal, 832(2), 99.

LOvis, C. \& FIsCHER, D. (2010) Radial Velocity Techniques for Exoplanets, chap. Part II. The University of Arizona Press, pp. 27-53.

Lovisi, L., Mucciarelli, A., Dalessandro, E., Ferraro, F. R. \& Lanzoni, B. (2013a) Another Brick in Understanding Chemical and Kinematical Properties of BSSs: NGC 6752. ApJ, 778(1), 64 .

Lovisi, L., Mucciarelli, A., Lanzoni, B., Ferraro, F. R., Dalessandro, E. \& Monaco, L. (2013b) Flames and XSHOOTER Spectroscopy along the Two Blue Straggler Star Sequences of M30. ApJ, 772(2), 148. 
Lucatello, S., Sollima, A., Gratton, R., Vesperini, E., D'Orazi, V., Carretta, E. \& BRAGAgLiA, A. (2015) The incidence of binaries in globular cluster stellar populations. $A \mathcal{E} A$, 584, A52.

LudendorfF, H. (1908) Über die Radialgeschwindigkeit der schwächeren Komponente von $\zeta$ Ursae majoris. Astronomische Nachrichten, 177(1), 7.

LÜtzgendorf, N., Kissler-Patig, M., Noyola, E., Jalali, B., De Zeeuw, P. T., GEBHARDT, K. \& BAUMGARDT, H. (2011a) Kinematic signature of an intermediate-mass black hole in the globular cluster NGC 6388. A\&A, 533, A36.

LÜtzgendorf, N., Kissler-Patig, M., Noyola, E., Jalali, B., De Zeeuw, P. T., GEBHARDT, K. \& BAUMGARDT, H. (2011b) Kinematic signature of an intermediate-mass black hole in the globular cluster NGC 6388. Astronomy and Astrophysics, 533, A36.

Maccarone, T. J., Kundu, A., ZepF, S. E. \& Rhode, K. L. (2007) A black hole in a globular cluster. Nature, 445, 183-185.

Mackey, A. D., Broby Nielsen, P., Ferguson, A. M. N. \& Richardson, J. C. (2008a) Multiple Stellar Populations in Three Rich Large Magellanic Cloud Star Clusters. ApJ, 681(1), L17.

Mackey, A. D., Wilkinson, M. I., Davies, M. B. \& Gilmore, G. F. (2007) The effect of stellar-mass black holes on the structural evolution of massive star clusters. MNRAS, 379(1), L40-L44.

Mackey, A. D., Wilkinson, M. I., Davies, M. B. \& Gilmore, G. F. (2008b) Black holes and core expansion in massive star clusters. MNRAS, 386(1), 65-95.

Malavolta, L., Piotto, G., Bedin, L. R., Sneden, C., Nascimbeni, V. \& Sommariva, V. (2015) The M 4 Core Project with HST - IV. Internal kinematics from accurate radial velocities of 2771 cluster members. Monthly Notices of the Royal Astronomical Society, 454(3), 2621-2635.

Malavolta, L., Sneden, C., Piotto, G., Milone, A. P., Bedin, L. R. \& Nascimbeni, V. (2014) Atmospheric Parameters and Metallicities for 2191 Stars in the Globular Cluster M4. The Astronomical Journal, 147(2), 25.

Mann, C. R., Richer, H., Heyl, J., Anderson, J., Kalirai, J., Caiazzo, I., Möhle, S. D., KneE, A. ET AL. (2019) A Multimass Velocity Dispersion Model of 47 Tucanae Indicates No Evidence for an Intermediate-mass Black Hole. ApJ, 875, 1.

Marino, A. F., Milone, A. P., Casagrande, L., Collet, R., Dotter, A., Johnson, C. I., LIND, K., BEDIN, L. R. ET AL. (2016) Chemical abundances in the multiple sub-giant branch of 47 Tucanae: insights on its faint sub-giant branch component. Monthly Notices of the Royal Astronomical Society, 459(1), 610-623. 
Marino, A. F., Milone, A. P., Karakas, A. I., Casagrand E, L., Yong, D., Shingles, L., DA COSTA, G., NORRIS, J. E. ET AL. (2015) Iron and s-elements abundance variations in NGC 5286: comparison with 'anomalous' globular clusters and Milky Way satellites. Monthly Notices of the Royal Astronomical Society, 450(1), 815-845.

Marino, A. F., Milone, A. P., Piotto, G., Villanova, S., Gratton, R., D’Antona, F., Anderson, J., Bedin, L. R. ET AL. (2011) Sodium-Oxygen Anticorrelation and Neutroncapture Elements in Omega Centauri Stellar Populations. The Astrophysical Journal, 731(1), 64.

Marino, A. F., Milone, A. P., Przybilla, N., Bergemann, M., Lind, K., Asplund, M., CASSISI, S., CATELAN, M. ET AL. (2014a) Helium enhanced stars and multiple populations along the horizontal branch of NGC 2808: direct spectroscopic measurements. Monthly Notices of the Royal Astronomical Society, 437(2), 1609-1627.

Marino, A. F., Milone, A. P., Sneden, C., Bergemann, M., Kraft, R. P., Wallerstein, G., CASSisI, S., APARICIO, A. ET AL. (2012) The double sub-giant branch of NGC 6656 (M 22): a chemical characterization. Astronomy and Astrophysics, 541, A15.

Marino, A. F., Milone, A. P., Yong, D., Dotter, A., Da Costa, G., Asplund, M., Jerjen, H., MACKEY, D. ET AL. (2014b) The halo+cluster system of the Galactic globular cluster NGC 1851. Monthly Notices of the Royal Astronomical Society, 442(4), 3044-3064.

Marino, A. F., Villanova, S., Piotto, G., Milone, A. P., Momany, Y., Bedin, L. R. \& Medling, A. M. (2008) Spectroscopic and photometric evidence of two stellar populations in the Galactic globular cluster NGC 6121 (M 4). Astronomy and Astrophysics, 490(2), 625-640.

Martocchia, S., Cabrera-Ziri, I., Lardo, C., Dalessandro, E., Bastian, N., Kozhurina-Platais, V., Usher, C., Niederhofer, F. ET Al. (2018) Age as a major factor in the onset of multiple populations in stellar clusters. MNRAS, 473, 2688-2700.

Massari, D., Fiorentino, G., McConnachie, A., Bono, G., Dall'Ora, M., Ferraro, I., IANNiCOla, G., Stetson, P. B. ET AL. (2016) GeMS MCAO observations of the Galactic globular cluster NGC 2808: the absolute age. Astronomy and Astrophysics, 586, A51.

Massari, D., Koppelman, H. H. \& Helmi, A. (2019) Origin of the system of globular clusters in the Milky Way. AEA, 630, L4.

Masuda, K. \& HotoKezaKa, K. (2019) Prospects of Finding Detached Black Hole-Star Binaries with TESS. ApJ, 883, 169.

McConnell, N. J. \& MA, C.-P. (2013) Revisiting the Scaling Relations of Black Hole Masses and Host Galaxy Properties. ApJ, 764(2), 184.

MCCREA, W. H. (1964) Extended main-sequence of some stellar clusters. MNRAS, 128, 147. 
McMillan, S. L. W., Pryor, C. \& Phinney, E. S. (1998) Binaries in Globular Clusters. Highlights of Astronomy, 11, 616.

MeYlan, G. \& Heggie, D. C. (1997) Internal dynamics of globular clusters. A\&A Rev., 8, 1-143.

MilleR, G. E. \& SCALO, J. M. (1979) The initial mass function and stellar birthrate in the solar neighborhood. ApJS, 41, 513-547.

Milone, A. P., Bedin, L. R., Piotto, G., Anderson, J., King, I. R., SARajedini, A., DotTER, A., Chaboyer, B. ET AL. (2008) The ACS Survey of Galactic Globular Clusters. III. The Double Subgiant Branch of NGC 1851. ApJ, 673(1), 241-250.

Milone, A. P., Marino, A. F., Bedin, L. R., Dotter, A., Jerjen, H., Kim, D., NArdiello, D., PiOtTO, G. ET AL. (2016) The binary populations of eight globular clusters in the outer halo of the Milky Way. MNRAS, 455(3), 3009-3019.

Milone, A. P., Marino, A. F., Piotto, G., Renzini, A., Bedin, L. R., Anderson, J., CasSISI, S., D'ANTONA, F. ET AL. (2015) The Hubble Space Telescope UV Legacy Survey of Galactic Globular Clusters. III. A Quintuple Stellar Population in NGC 2808. ApJ, 808, 51.

Milone, A. P., Piotto, G., Bedin, L. R., Aparicio, A., Anderson, J., Sarajedini, A., MARINO, A. F., MoretTI, A. ET AL. (2012) The ACS survey of Galactic globular clusters. XII. Photometric binaries along the main sequence. A\&A, 540, A16.

Milone, A. P., Piotto, G., Renzini, A., Marino, A. F., Bedin, L. R., Vesperini, E., D'AntonA, F., NARDiello, D. ET AL. (2017) The Hubble Space Telescope UV Legacy Survey of Galactic globular clusters - IX. The Atlas of multiple stellar populations. MNRAS, 464(3), 3636-3656.

Minniti, D., Contreras Ramos, R., Alonso-García, J., Anguita, T., Catelan, M., Gran, F., MotTA, V., Muro, G. ET AL. (2015) VVV Survey Observations of a Microlensing Stellar Mass Black Hole Candidate in the Field of the Globular Cluster NGC 6553. ApJ, 810(2), L20.

MochejSKa, B. J., KAluZnY, J., ThOMPsOn, I. \& PYCH, W. (2002) Clusters Ages Experiment: Hot Subdwarfs and Luminous White Dwarf Candidates in the Field of the Globular Cluster M4. The Astronomical Journal, 124(3), 1486-1496.

Momany, Y., Saviane, I., Smette, A., Bayo, A., Girardi, L., Marconi, G., Milone, A. P. \& BRESSAN, A. (2012) The VLT/VISIR mid-IR view of 47 Tucanae. A further step in solving the puzzle of RGB mass loss. Astronomy and Astrophysics, 537, A2.

Moni Bidin, C., Villanova, S., Piotto, G. \& Momany, Y. (2011) A lack of close binaries among hot horizontal branch stars in globular clusters. II. NGC 2808. Astronomy and Astrophysics, 528, A127. 
Morscher, M., Pattabiraman, B., Rodriguez, C., Rasio, F. A. \& Umbreit, S. (2015) The Dynamical Evolution of Stellar Black Holes in Globular Clusters. ApJ, 800, 9.

Mucciarelli, A., Lapenna, E., Massari, D., Ferraro, F. R. \& Lanzoni, B. (2015) The Origin of the Spurious Iron Spread in the Globular Cluster NGC 3201. The Astrophysical Journal, 801(1), 69.

Mucciarelli, A., Lovisi, L., Ferraro, F. R., Dalessandro, E., Lanzoni, B. \& Monaco, L. (2014) Spinning Like a Blue Straggler: The Population of Fast Rotating Blue Straggler Stars in $\omega$ Centauri. ApJ, 797, 43.

Nardiello, D., libralato, M., Piotto, G., Anderson, J., Bellini, A., Aparicio, A., Bedin, L. R., CASsisI, S. ET AL. (2018) The Hubble Space Telescope UV Legacy Survey of Galactic Globular Clusters - XVII. Public Catalogue Release. MNRAS, 481, 3382-3393.

Nascimbeni, V., Bedin, L. R., Heggie, D. C., van den Berg, M., Giersz, M., Piotto, G., BrogaArd, K., Bellini, A. ET AL. (2014) The M 4 Core Project with HST - III. Search for variable stars in the primary field. Monthly Notices of the Royal Astronomical Society, 442(3), 2381-2391.

Navarrete, C., Catelan, M., Contreras Ramos, R., Alonso-García, J., Gran, F., DÉKÁNY, I. \& MiNNITI, D. (2017) Near-IR period-luminosity relations for pulsating stars in $\omega$ Centauri (NGC 5139). Astronomy and Astrophysics, 604, A120.

Neeley, J. R., Marengo, M., Bono, G., Braga, V. F., Dall'Ora, M., Stetson, P. B., BuONANNO, R., FerRaro, I. ET AL. (2015) On the Distance of the Globular Cluster M4 (NGC 6121) Using RR Lyrae Stars. II. Mid-infrared Period-luminosity Relations. The Astrophysical Journal, 808(1), 11 .

Norris, J. E., Freeman, K. C. \& Mighell, K. J. (1996) The Giant Branch of omega Centauri. V. The Calcium Abundance Distribution. The Astrophysical Journal, 462, 241.

Pancino, E., Galfo, A., Ferraro, F. R. \& Bellazzini, M. (2007) The Rotation of Subpopulations in $\omega$ Centauri. The Astrophysical Journal, 661(2), L155-L158.

Peebles, P. J. E. (1972) Star Distribution Near a Collapsed Object. ApJ, 178, 371-376.

Peterson, R. C., Rees, R. F. \& Cudworth, K. M. (1995) Radial Velocities of Stars in the Globular Cluster M4 and the Cluster Distance. The Astrophysical Journal, 443, 124.

Pfeffer, J., Kruijssen, J. M. D., Crain, R. A. \& Bastian, N. (2018) The E-MOSAiCS project: simulating the formation and co-evolution of galaxies and their star cluster populations. MNRAS, 475(4), 4309-4346.

Pickering, E. C. (1890) On the spectrum of zeta Ursae Majoris. The Observatory, 13, 80-81. 
Piotto, G., Bedin, L. R., Anderson, J., King, I. R., Cassisi, S., Milone, A. P., VillanOva, S., Pietrinferni, A. ET AL. (2007) A Triple Main Sequence in the Globular Cluster NGC 2808. ApJ, 661(1), L53-L56.

Piotto, G., Milone, A. P., Bedin, L. R., Anderson, J., King, I. R., Marino, A. F., NARdiEllo, D., Aparicio, A. ET AL. (2015) The Hubble Space Telescope UV Legacy Survey of Galactic Globular Clusters. I. Overview of the Project and Detection of Multiple Stellar Populations. $A J, 149,91$.

Piotto, G., Villanova, S., Bedin, L. R., Gratton, R., Cassisi, S., Momany, Y., RecioBlanco, A., Lucatello, S. ET Al. (2005) Metallicities on the Double Main Sequence of $\omega$ Centauri Imply Large Helium Enhancement. ApJ, 621(2), 777-784.

Pollard, D. L., SAndquist, E. L., Hargis, J. R. \& Bolte, M. (2005) The Unusual Luminosity Function of the Globular Cluster M10. The Astrophysical Journal, 628(2), 729-737.

Pooley, D. \& Hut, P. (2006) Dynamical Formation of Close Binaries in Globular Clusters: Cataclysmic Variables. ApJ, 646, L143-L146.

Pooley, D., Lewin, W. H. G., Anderson, S. F., Baumgardt, H., Filippenko, A. V., Gaensler, B. M., Homer, L., Hut, P. ET AL. (2003) Dynamical Formation of Close Binary Systems in Globular Clusters. ApJ, 591, L131-L134.

Price-Whelan, A. M., Hogg, D. W., Foreman-Mackey, D. \& Rix, H.-W. (2017) The Joker: A Custom Monte Carlo Sampler for Binary-star and Exoplanet Radial Velocity Data. ApJ, 837, 20.

Pryor, C. \& Meylan, G. (1993) Velocity Dispersions for Galactic Globular Clusters. In Structure and Dynamics of Globular Clusters, edited by S. G. Djorgovski \& G. Meylan, vol. 50 of Astronomical Society of the Pacific Conference Series.

Pryor, C. P., Latham, D. W. \& Hazen, M. L. (1988) A search for spectroscopic binaries in the globular cluster M3. AJ, 96, 123-138.

Rees, Richard F., J. (1993) New Proper Motions in the Globular Cluster M5. The Astronomical Journal, 106, 1524.

Rodriguez, C. L., Amaro-Seoane, P., Chatterjee, S., Kremer, K., Rasio, F. A., SamSING, J., YE, C. S. \& ZEVIN, M. (2018) Post-Newtonian dynamics in dense star clusters: Formation, masses, and merger rates of highly-eccentric black hole binaries. Phys. Rev. D, 98(12), 123005.

Roederer, I. U., MARino, A. F. \& Sneden, C. (2011) Characterizing the Heavy Elements in Globular Cluster M22 and an Empirical s-process Abundance Distribution Derived from the Two Stellar Groups. The Astrophysical Journal, 742(1), 37. 
Roth, M. M., Kelz, A., Fechner, T., Hahn, T., BAuer, S.-M., Becker, T., BÖHM, P., Christensen, L. ET AL. (2005) PMAS: The Potsdam Multi-Aperture Spectrophotometer. I. Design, Manufacture, and Performance. PASP, 117, 620-642.

Ruiter, A. J., Ferrario, L., Belczynski, K., Seitenzahl, I. R., Crocker, R. M. \& KaraKAS, A. I. (2019) On the formation of neutron stars via accretion-induced collapse in binaries. MNRAS, 484(1), 698-711.

Salgado, C., Moni Bidin, C., Villanova, S., Geisler, D. \& Catelan, M. (2013) Spectroscopy of blue horizontal branch stars in NGC 6656 (M 22). Astronomy and Astrophysics, 559, A101.

SALPETER, E. E. (1955) The Luminosity Function and Stellar Evolution. ApJ, 121, 161.

SANDAGE, A. R. (1953) The color-magnitude diagram for the globular cluster M 3. AJ, 58, 61-75.

SANDquist, E. L. \& Bolte, M. (2004) Exploring the Upper Red Giant and Asymptotic Giant Branches: The Globular Cluster M5. The Astrophysical Journal, 611(1), 323-337.

Sarajedini, A., Bedin, L. R., Chaboyer, B., Dotter, A., Siegel, M., Anderson, J., AparICIO, A., KING, I. ET AL. (2007) The ACS Survey of Galactic Globular Clusters. I. Overview and Clusters without Previous Hubble Space Telescope Photometry. AJ, 133, 1658-1672.

SARIYA, D. P., JiAnG, I.-G. \& YADAV, R. K. S. (2017) A Proper Motions Study of the Globular Cluster NGC 3201. The Astronomical Journal, 153(3), 134.

Scarpa, R., Marconi, G., Carraro, G., Falomo, R. \& Villanova, S. (2011) Testing Newtonian gravity with distant globular clusters: NGC 1851 and NGC 1904. Astronomy and Astrophysics, 525, A148.

SCARPa, R., MARConi, G., Gilmozzi, R. \& CARRARO, G. (2007) Using globular clusters to test gravity in the weak acceleration regime: NGC 7099. Astronomy and Astrophysics, 462(1), L9-L12.

SCHECK, F. (2006) Theoretische Physik 3 Klassische Feldtheorie. Springer.

SCHUltz, H. (1866) Historische Notizen über Nebelflecke. Astronomische Nachrichten, 67(1585), $1-6$.

SCHWARZSCHILD, K. (1916) Über das Gravitationsfeld eines Massenpunktes nach der Einsteinschen Theorie. Sitzungsberichte der Königlich Preußischen Akademie der Wissenschaften (Berlin, 189-196.

SEARLE, L. \& ZINN, R. (1978) Compositions of halo clusters and the formation of the galactic halo. ApJ, 225, 357-379. 
SHAPLEY, H. (1916) Studies based on the colors and magnitudes in stellar clusters. I. The general problem of clusters. Contributions from the Mount Wilson Observatory / Carnegie Institution of Washington, 115, 1.

Sigurdsson, S. \& Hernquist, L. (1993) Primordial black holes in globular clusters. Nature, 364(6436), 423-425.

Simpson, J. D., Cottrell, P. L. \& Worley, C. C. (2012) Spectral matching for abundances and clustering analysis of stars on the giant branches of $\omega$ Centauri. Monthly Notices of the Royal Astronomical Society, 427(2), 1153-1167.

Simunovic, M. \& PuZiA, T. H. (2014) Blue Straggler Star Populations in Globular Clusters. I. Dynamical Properties of Blue Straggler Stars in NGC 3201, NGC 6218, and $\omega$ Centauri. ApJ, $782,49$.

SKUmANICH, A. (1972) Time Scales for CA II Emission Decay, Rotational Braking, and Lithium Depletion. ApJ, 171, 565.

Sollima, A., Beccari, G., Ferraro, F. R., Fusi Pecci, F. \& Sarajedini, A. (2007a) The fraction of binary systems in the core of 13 low-density Galactic globular clusters. MNRAS, 380, 781-791.

Sollima, A., Borissova, J., Catelan, M., Smith, H. A., Minniti, D., Cacciari, C. \& FERRARO, F. R. (2006) New Metallicities of RR Lyrae Stars in $\omega$ Centauri: Evidence for a Non-He-enhanced Metal-intermediate Population. The Astrophysical Journal, 640(1), L43-L46.

Sollima, A., Ferraro, F. R., Bellazzini, M., Origlia, L., Straniero, O. \& Pancino, E. (2007b) Deep FORS1 Observations of the Double Main Sequence of $\omega$ Centauri. ApJ, 654, 915-922.

Sollima, A., Ferraro, F. R., Origlia, L., Pancino, E. \& Bellazzini, M. (2004) IR photometric properties of Red Giants in $\omega$ Cen. Astronomy and Astrophysics, 420, 173-181.

Sollima, A., Ferraro, F. R., Pancino, E. \& Bellazzini, M. (2005) On the discrete nature of the red giant branch of $\omega$ Centauri. Monthly Notices of the Royal Astronomical Society, 357(1), $265-274$.

Sollima, A., Lanzoni, B., Beccari, G., Ferraro, F. R. \& Fusi Pecci, F. (2008) The correlation between blue straggler and binary fractions in the core of Galactic globular clusters. $A \mathcal{E} A, 481,701-704$.

Soto, M., Bellini, A., Anderson, J., Piotto, G., Bedin, L. R., VAn Der Marel, R. P., Milone, A. P., Brown, T. M. ET AL. (2017) The Hubble Space Telescope UV Legacy Survey of Galactic Globular Clusters. VIII. Preliminary Public Catalog Release. AJ, 153, 19. 
SPITZER, JR., L. (1969) Equipartition and the Formation of Compact Nuclei in Spherical Stellar Systems. ApJ, 158, L139.

SPURZEM, R. \& AARSETH, S. J. (1996) Direct collisional simulation of 10000 particles past core collapse. MNRAS, 282, 19.

SPURZEM, R. \& GIERSZ, M. (1996) A stochastic Monte-Carlo approach to model real star cluster evolution. I. The model. MNRAS, 283, 805.

Stetson, P. B., Braga, V. F., Dall'Ora, M., Bono, G., Buonanno, R., Ferraro, I., IANNICOLA, G., MARENGO, M. ET AL. (2014) Optical and Near-Infrared UBVRIJHK Photometry for the RR Lyrae Stars in the Nearby Globular Cluster M4 (NGC 6121). Publications of the Astronomical Society of the Pacific, 126(940), 521.

Strader, J., Chomiuk, L., Maccarone, T. J., Miller-Jones, J. C. A. \& Seth, A. C. (2012) Two stellar-mass black holes in the globular cluster M22. Nature, 490, 71-73.

Ströbele, S., la Penna, P., Arsenault, R., Conzelmann, R. D., Delabre, B., DuchATEAU, M., Dorn, R., Fedrigo, E. ET AL. (2012) GALACSI system design and analysis. In Adaptive Optics Systems III, vol. 8447 of Proc. SPIE.

StRÖMGREN, B. (1933) On the Interpretation of the Hertzsprung-Russell-Diagram. Mit $4 \mathrm{Ab}-$ bildungen. $Z A p, 7,222$.

STRYKER, L. L. (1993) Blue Stragglers. PASP, 105, 1081.

SzéKely, P., Kiss, L. L., Jackson, R., Derekas, A., CSÁK, B. \& Szatmáry, K. (2007) RR Lyrae stars in the southern globular cluster NGC 362. Astronomy and Astrophysics, 463(2), 589600 .

TAURIS, T. M. \& VAN DEN HeUVEL, E. P. J. (2006) Formation and evolution of compact stellar X-ray sources. Cambridge University Press, pp. 623-665.

Thompson, T. A., Kochanek, C. S., Stanek, K. Z., Badenes, C., Post, R. S., JaYASinghe, T., LATHAM, D. W., Bieryla, A. ET AL. (2019) A noninteracting low-mass black hole $[\mathrm{U}+2500$ ] giant star binary system. Science, 366(6465), 637-640.

Thomson, G. S., Knigge, C., Dieball, A., Maccarone, T. J., Dolphin, A., Zurek, D., LONG, K. S., SHARA, M. ET AL. (2012) A multiwavelength survey of NGC 6752: X-ray counterparts, two new dwarf novae and a core-collapsed radial profile. Monthly Notices of the Royal Astronomical Society, 423(3), 2901-2916.

Tremou, E., Strader, J., Chomiuk, L., Shishkovsky, L., Maccarone, T. J., MillerJones, J. C. A., Tudor, V., Heinke, C. O. ET Al. (2018) The MAVERIC Survey: Still No Evidence for Accreting Intermediate-mass Black Holes in Globular Clusters. ApJ, 862, 16. 
Trujillo, I., Beasley, M. A., Borlaff, A., Carrasco, E. R., Di Cintio, A., Filho, M., Monelli, M., Montes, M. ET AL. (2019) A distance of 13 Mpc resolves the claimed anomalies of the galaxy lacking dark matter. MNRAS, 486, 1192-1219.

VAN DEN Heuvel, E. P. J. (1967) The origin of blue stragglers and peculiar and metallic-line stars. The Observatory, 87, 68-72.

VAN Loon, J. T., VAN Leeuwen, F., SMalley, B., Smith, A. W., Lyons, N. A., McDonald, I. \& BOYER, M. L. (2007) A spectral atlas of post-main-sequence stars in $\omega$ Centauri: kinematics, evolution, enrichment and interstellar medium. Monthly Notices of the Royal Astronomical Society, 382(3), 1353-1374.

Vesperini, E., McMillan, S. L. W., D'Antona, F. \& D'Ercole, A. (2010) The Fraction of Globular Cluster Second-generation Stars in the Galactic Halo. ApJ, 718, L112-L116.

Villanova, S., Geisler, D., Gratton, R. G. \& Cassisi, S. (2014) The Metallicity Spread and the Age-Metallicity Relation of $\omega$ Centauri. The Astrophysical Journal, 791(2), 107.

Vivas, A. K., Saha, A., Olsen, K., Blum, R., Olszewski, E. W., Claver, J., Valdes, F., AXELROD, T. ET AL. (2017) Absolute Magnitudes and Colors of RR Lyrae Stars in DECam Passbands from Photometry of the Globular Cluster M5. The Astronomical Journal, 154(3), 85.

VOlOnTERI, M. (2012) The Formation and Evolution of Massive Black Holes. Science, 337, 544.

von Braun, K. \& MAteO, M. (2002) Variable Stars in the Field of the Globular Cluster NGC 3201. The Astronomical Journal, 123(1), 279-289.

Walker, M. G., Mateo, M., Olszewski, E. W., Sen, B. \& Woodroofe, M. (2009) Clean Kinematic Samples in Dwarf Spheroidals: An Algorithm for Evaluating Membership and Estimating Distribution Parameters When Contamination is Present. AJ, 137(2), 3109, URL http://stacks.iop.org/1538-3881/137/i=2/a=3109.

Wang, L., Spurzem, R., Aarseth, S., Giersz, M., Askar, A., Berczik, P., NaAb, T., SCHADOW, R. ET AL. (2016a) The DRAGON simulations: globular cluster evolution with a million stars. MNRAS, 458, 1450-1465.

Wang, Y., Primas, F., Charbonnel, C., Van Der SWaelmen, M., Bono, G., ChanterEAU, W. \& ZHAO, G. (2016b) Sodium abundances of AGB and RGB stars in Galactic globular clusters. I. Analysis and results of NGC 2808. Astronomy and Astrophysics, 592, A66.

Wang, Y., Primas, F., Charbonnel, C., Van der Swaelmen, M., Bono, G., ChanterEAU, W. \& ZHAO, G. (2017) Sodium abundances of AGB and RGB stars in Galactic globular clusters. II. Analysis and results of NGC 104, NGC 6121, and NGC 6809. Astronomy and Astrophysics, 607, A135. 
Warren, S. R., SAndquist, E. L. \& Bolte, M. (2006) The Blue Straggler Population of the Globular Cluster M5: Comparison with M3. The Astrophysical Journal, 648(2), 1026-1036.

Weilbacher, P. M., Streicher, O., Urrutia, T., Jarno, A., Pécontal-Rousset, A., BACON, R. \& BÖHM, P. (2012) Design and capabilities of the MUSE data reduction software and pipeline. In Software and Cyberinfrastructure for Astronomy II, vol. 8451 of Proc. SPIE.

Weilbacher, P. M., Streicher, O., Urrutia, T., Pécontal-Rousset, A., Jarno, A. \& BACON, R. (2014) The MUSE Data Reduction Pipeline: Status after Preliminary Acceptance Europe. In Astronomical Data Analysis Software and Systems XXIII, edited by N. Manset \& P. Forshay, vol. 485 of Astronomical Society of the Pacific Conference Series.

Wendt, M., Husser, T.-O., Kamann, S., Monreal-Ibero, A., Richter, P., Brinchmann, J., Dreizler, S., WeIlbacher, P. M. ET AL. (2017) Mapping diffuse interstellar bands in the local ISM on small scales via MUSE 3D spectroscopy. A pilot study based on globular cluster NGC 6397. A\&A, 607, A133.

WILliams, I. P. (1964) Possible spread in the ages of stars in a stellar cluster. MNRAS, 128, 389.

Wise, J. H., Regan, J. A., O'Shea, B. W., Norman, M. L., Downes, T. P. \& XU, H. (2019) Formation of massive black holes in rapidly growing pre-galactic gas clouds. Nature, 566, $85-88$.

Yalinewich, A., Beniamini, P., HotokezaKa, K. \& ZhU, W. (2018) Dark passengers in stellar surveys. MNRAS, 481, 930-937.

YONG, D., GRUNDAHL, F. \& NORRIS, J. E. (2015) CNO abundances in the globular clusters NGC 1851 and NGC 6752. Monthly Notices of the Royal Astronomical Society, 446(4), 3319-3329.

Zepf, S. E. \& Ashman, K. M. (1993) Globular Cluster Systems Formed in Galaxy Mergers. MNRAS, 264, 611 .

ZINN, R. (1985) The globular cluster system of the galaxy. IV - The halo and disk subsystems. ApJ, 293, 424-444.

ZloczewsKi, K., Kaluzny, J., RozyczKa, M., KRZeminsKi, W. \& MaZuR, B. (2012) A Proper Motion Study of the Globular Clusters M4, M12, M22, NGC 3201, NGC 6362 and NGC 6752. Acta Astronomica, 62(4), 357-375.

Zorotovic, M., Catelan, M., Smith, H. A., Pritzl, B. J., Aguirre, P., Angulo, R. E., Aravena, M., Assef, R. J. ET AL. (2010) The Globular Cluster NGC 5286. II. Variable Stars. The Astronomical Journal, 139(2), 357-371. 